\title{
Investigating the mode of action of tuberculosis drugs using hypersensitive mutants of Mycobacterium smegmatis
}

by

\section{Richard Laurence Campen}

BSc(Hons.) Victoria University of Wellington

\begin{abstract}
A thesis submitted to the Victoria University of Wellington in fulfilment of the requirements for the degree of Doctor of Philosophy in Molecular Microbiology
\end{abstract}

Victoria University of Wellington 



\section{Primary Supervisor}

Dr Ronan O'Toole

Senior Lecturer, School of Medicine

University of Tasmania

\section{Victoria Supervisor}

Professor John H. Miller

School of Biological Sciences

Victoria University of Wellington 



\begin{abstract}
Mycobacterium tuberculosis, the etiological agent of tuberculosis (TB), is the leading cause of death and disease by a bacterial pathogen worldwide. The growing incidence of drug resistant TB, especially multi-drug resistant TB highlights the need for new drugs with novel modes of action. Current treatment of TB involves a multi-drug regimen of four drugs including isoniazid and rifampicin, both of which were discovered over 40 years ago. Bedaquiline is one of the first novel TB drugs to enter clinical trials since the discovery of rifampicin, and has shown excellent activity against drug resistant TB. Although isoniazid and rifampicin are well established anti-TB drugs, significant gaps in knowledge regarding their modes of action exist. Furthermore, little information on the mode of action of the novel drug bedaquiline is known beyond its primary target. Characterisation of drug mode of action facilitates rational modifications of drugs to improve the treatment of TB.
\end{abstract}

The aim of this study was to identify novel aspects of the modes of action of isoniazid, rifampicin, and bedaquiline by characterising drug hypersensitive mutants of $M$. smegmatis $\mathrm{mc}^{2}$ 155. A sub-saturated $M$. smegmatis $\mathrm{mc}^{2} 155$ transposon mutant collection with 1.1-fold genome coverage (7680 mutants) was constructed, with this collection estimated to contain mutations in $73.2 \%$ of all genes capable of maintaining a transposon insertion (non-essential genes). A high-throughput assay was developed for screening the collection, and mutants related to known drug mode of action were identified for isoniazid ( $a h p C$ and eccCa $)_{1}$ and bedaquiline $(a t p B)$. Additionally, known mechanisms of drug inactivation were identified for isoniazid (nudC), rifampicin ( $\operatorname{arr}$ and IspA), and bedaquiline (mmpL5). The finding that transposon mutants of $n u d C$ are hypersensitive to isoniazid independently validated the recent discovery of the role of NudC in basal isoniazid resistance by Wang et al. (2011). The remaining genes identified in this thesis represent potentially novel aspects of the modes of action or resistance mechanisms of these drugs.

Cross-sensitivity to other drugs indicated that the mechanism of sensitivity was drug specific for the mutants examined. Differential-sensitivity testing against drug analogues revealed that Arr is involved in resistance to the rifampicin analogue rifapentine as well, indicating that Arr can detoxify rifapentine similar to rifampicin. The nudC mutant showed increased sensitivity to a range of isoniazid analogues, indicating that it can detoxify these analogues 
similar to the parent compound. Interestingly six analogues were found to be less active against the nudC mutant than expected. A number of overexpression strains were tested against these six analogues; a nudC overexpression strain, and a strain overexpressing $\operatorname{inh} A$, the primary target for isoniazid. Overexpression of nudC as well inhA increased the resistance of WT to isoniazid, but failed to increase resistance to three of the analogues, NSC27607, NSC33759, and NSC40350. Isoniazid is a prodrug and is activated by the peroxidase/catalase enzyme KatG. Overexpression of katG resulted in increased isoniazid sensitivity, as well as increased sensitivity to NSC27607, NSC33759, and NSC40350. Together these results suggest that NSC27607, NSC33759, and NSC40350 are activated by KatG, but that InhA is not the primary target. Additionally the inability of NudC overexpression to confer resistance suggests these analogues are not acting via a NAD adduct, the mechanism by which isoniazid inhibits InhA. These results suggest that there are other toxic metabolites being produced by KatG activation of these three analogues.

In conclusion, characterisation of mutants identified in a high-throughput assay for drug hypersensitivity identified genes involved in the modes of action or resistance mechanisms for isoniazid, rifampicin, and bedaquiline. Additionally, a number of novel genes were identified that have no known connections to the known modes of action or resistance mechanisms for these drugs. Further testing of a nudC mutant revealed three isoniazid analogues that appear to inhibit growth of $M$. smegmatis $\mathrm{mc}^{2} 155$ independent of $\operatorname{Inh} A$, the primary target of isoniazid. This study has successfully demonstrated that screening for drug hypersensitivity can generate novel information on drug mode of action and resistance mechanisms. This information can ultimately be used to help drive the development of new drugs, and improve treatment of TB. 


\section{Acknowledgements}

First I would like to thank my primary supervisor Ronan O'Toole for taking me on as a PhD student, and thank you for your support and guidance throughout my project. I would also like to thank my Victoria supervisor Professor John Miller for his support during the later stages of my PhD project, particularly with proof reading my thesis. My gratitude also goes to Associate Professor David Ackerley for taking me in during the later stages of my PhD. Inclusion in your lab group helped me get through the last few years of my project. Thank you also for your help with proof reading my thesis. Thank you also to Dr Joanna Mackichan for sharing her lab space during the last few years of my PhD.

I am also grateful to the fellow O'Toole lab students over the years, Shahista Nisa, Chris Miller, Nathaniel Dasyam, Ian Bassett, Mudassar Altaf, and Sandi Dempsy. Your help and guidance over the years was invaluable. My gratitude also to Yee Suen Low for her help with practical aspects of my project. Thank you also to the Ackerley lab group, including long time office mates Mark Calcott, Katherine Robins, and Becky Edgar, as well as the rest of KK815/816 for their support and friendship throughout my PhD.

Thank you also to our collaborator Professor Greg Cook at the University of Otago, and his post-doc Jen Robson. Thank you for providing a sample of bedaquiline for this study, and for your help with the practical aspects of the bedaquiline assay. Thank you also for flying me down to Otago to discuss my project, and present my work to your research group.

Thank you to my wonderful family and friends for all their support throughout my PhD. Especially thank you to my mother and father for their emotional and financial support, and to my father for sharing his roof with us for the last couple years. Finally, thank you to my amazing wife Kelly. Thank you so much for your love and support, it has been instrumental in me completing this project. 


\section{Table of Contents}

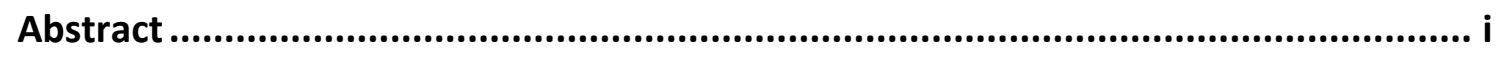

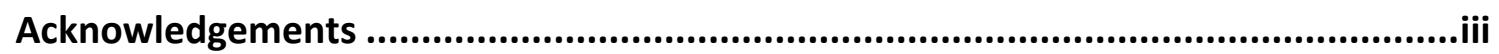

Table of Contents...........................................................................................................

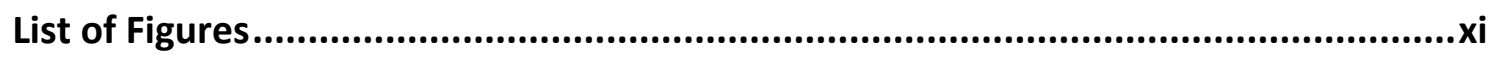

List of Tables ...............................................................................................

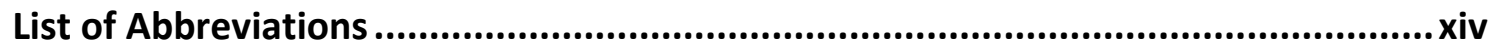

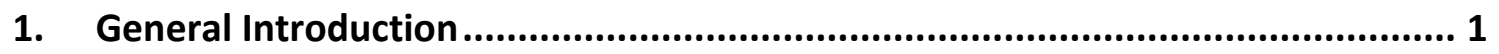

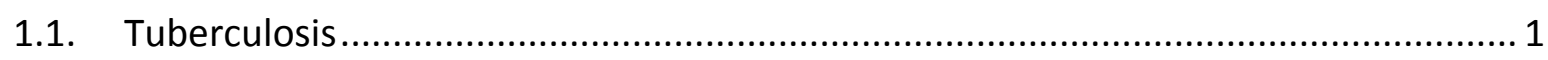

1.1.1. Tuberculosis in New Zealand .................................................................... 4

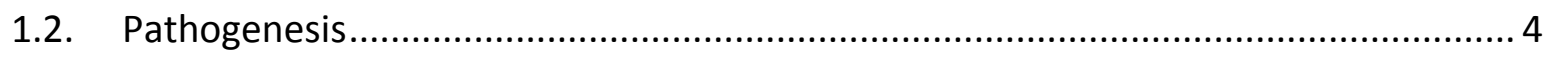

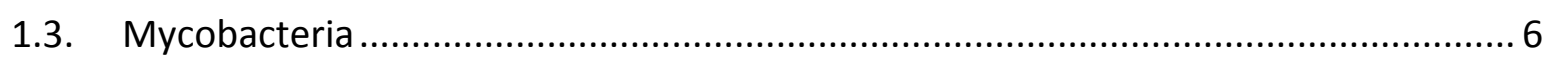

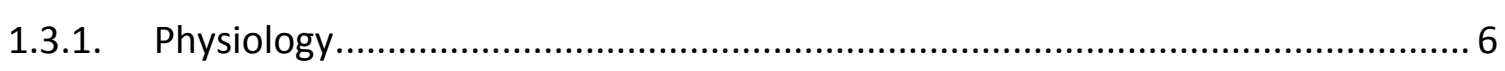

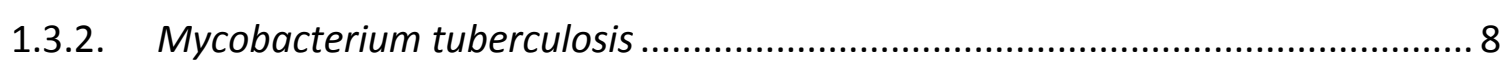

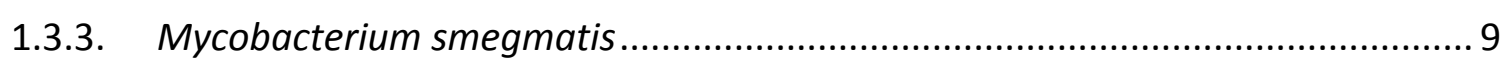

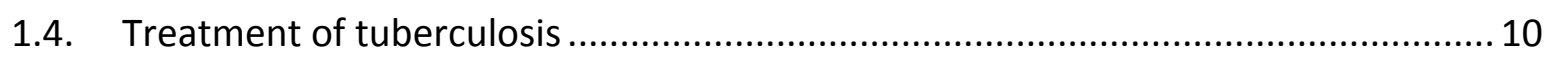

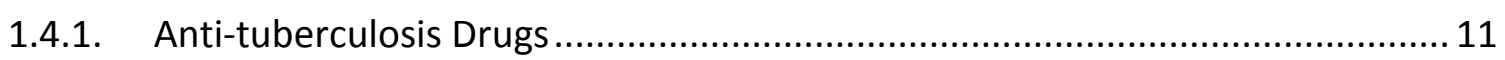

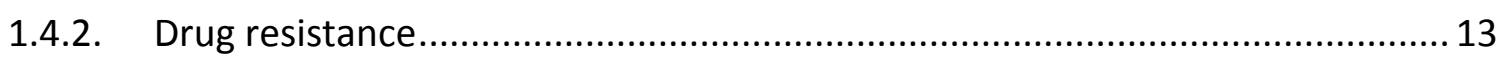

1.4.3. Modes of action of the anti-tuberculosis drugs to be examined in this study.. 13

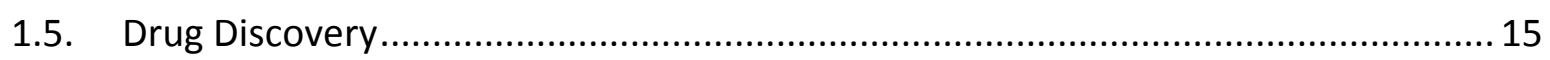

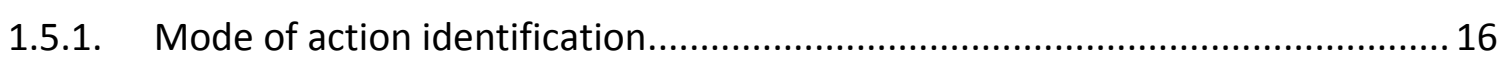

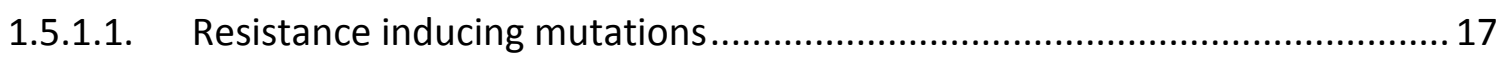

1.5.1.2. Altered transcription with drug treatment ............................................. 17

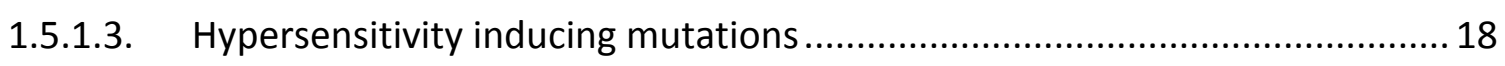

1.6. Random transposon mutagenesis of $M$. smegmatis ........................................... 19

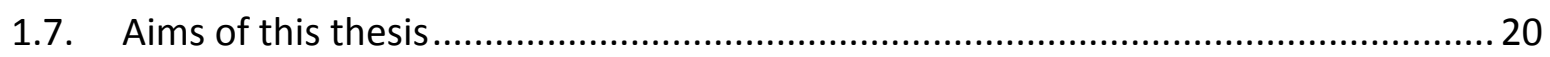

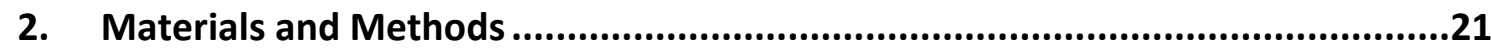

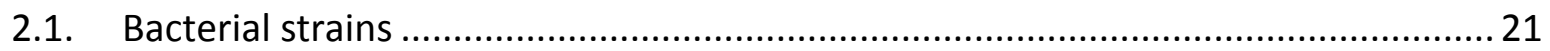

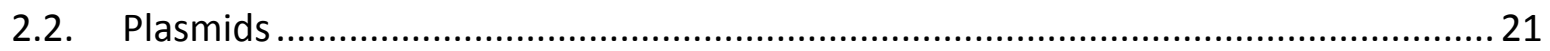

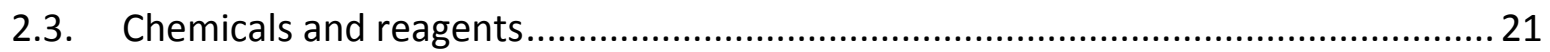

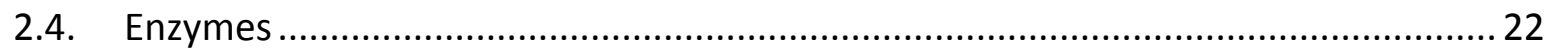




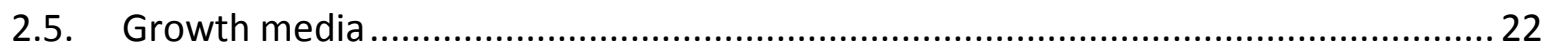

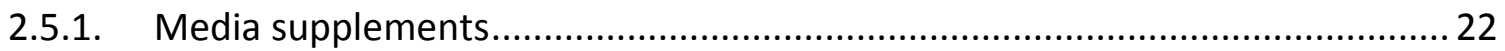

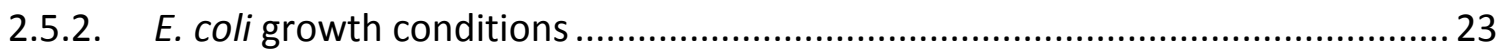

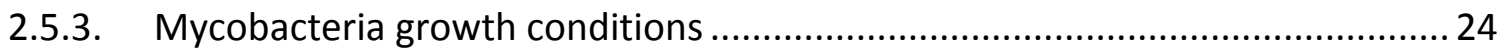

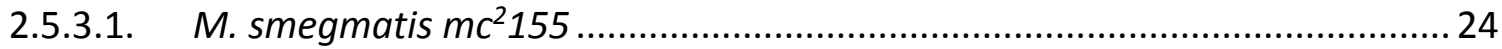

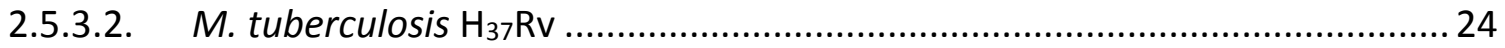

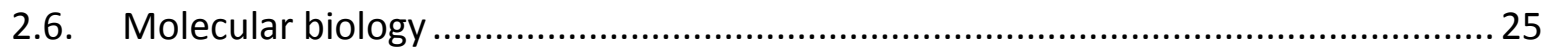

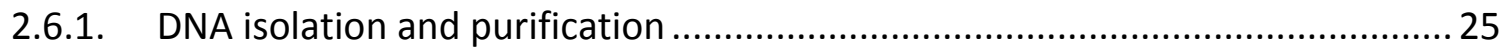

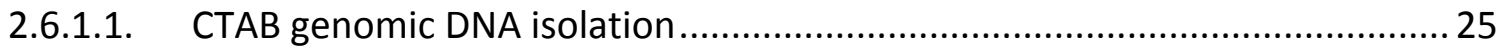

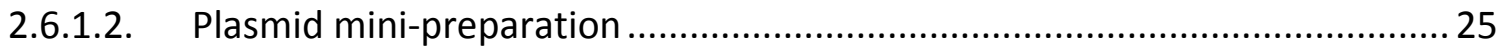

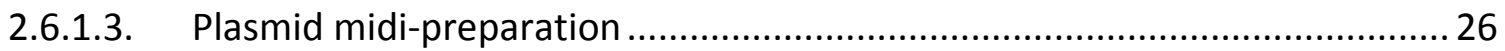

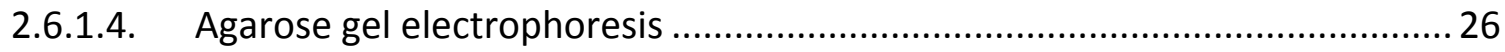

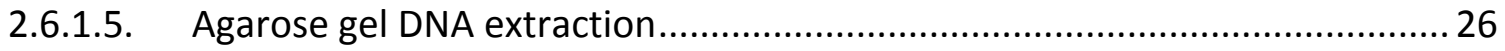

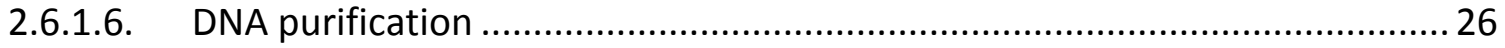

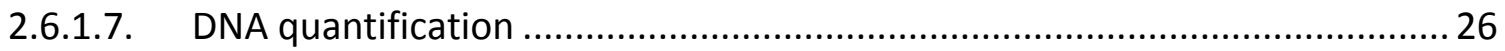

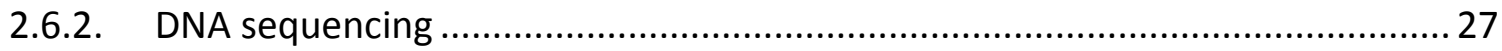

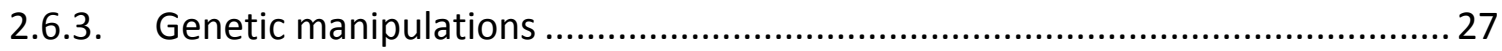

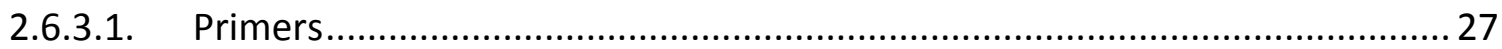

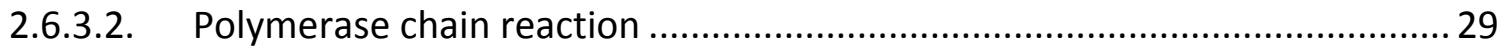

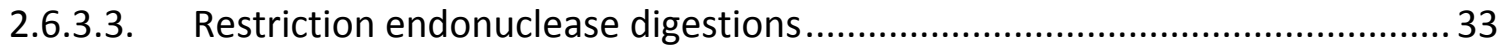

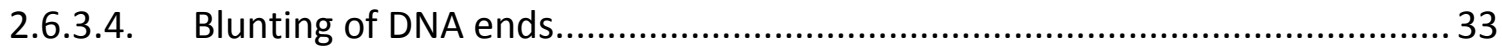

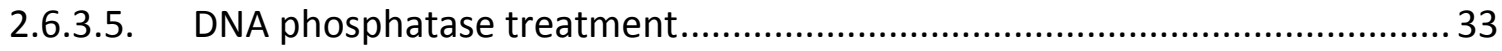

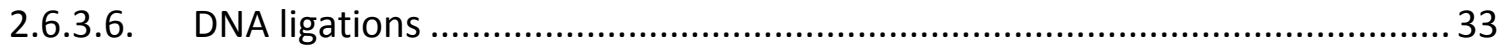

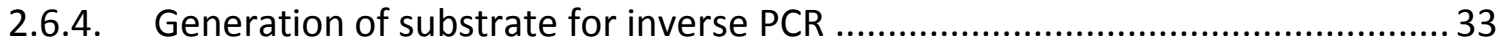

2.6.5. Generation of substrate for ligation mediated PCR ......................................... 34

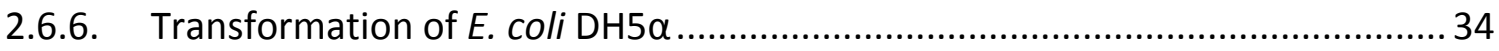

2.6.6.1. Generation of chemically competent $E$. coli cells ....................................... 34

2.6.6.2. Heat shock transformation of $E$. coli cells ................................................... 35

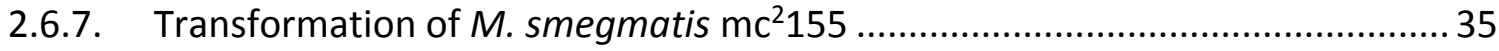

2.6.7.1. Generation of electrocompetant M. smegmatis cells.................................. 35

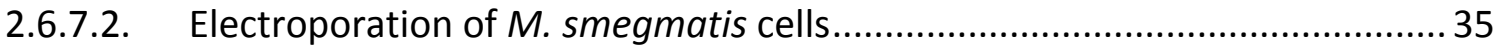

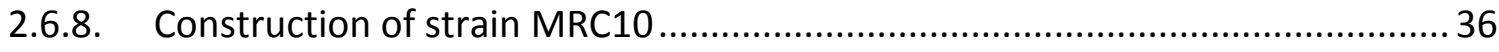

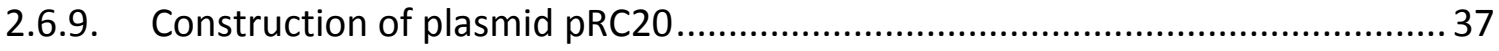

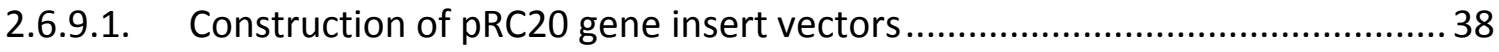

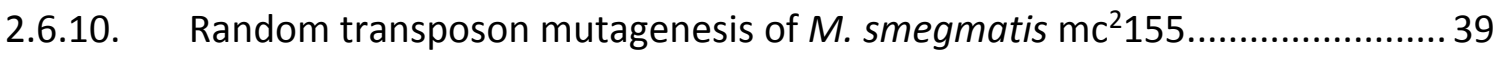




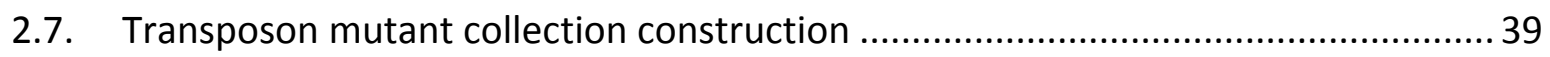

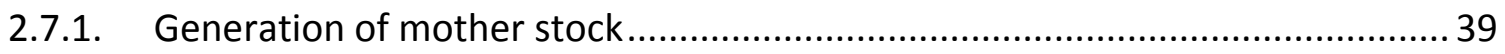

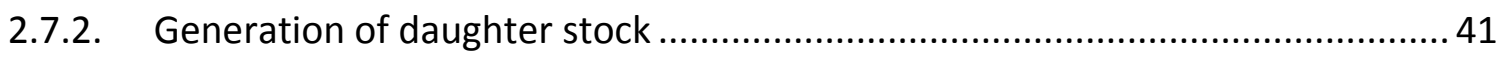

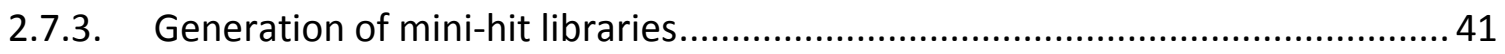

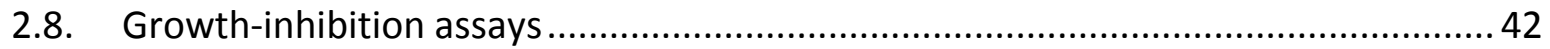

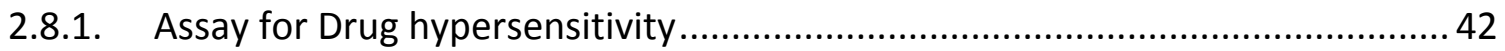

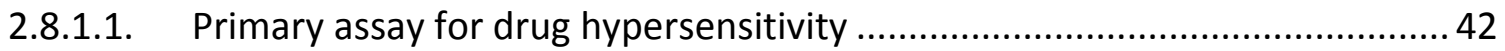

2.8.1.2. Secondary assay for drug-hypersensitivity ................................................. 44

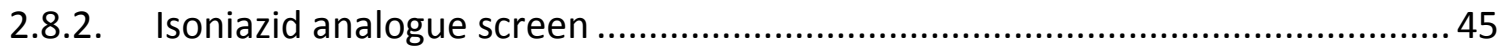

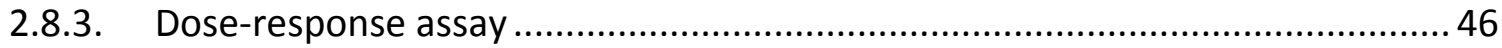

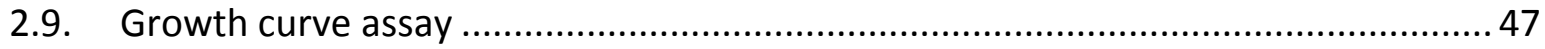

3. Isolation of drug hypersensitive mutants of $M$. smegmatis $\mathrm{mc}^{2} 155 \ldots \ldots \ldots \ldots \ldots . . . . .49$

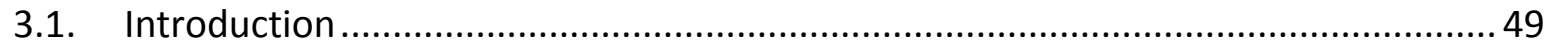

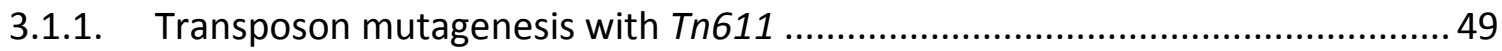

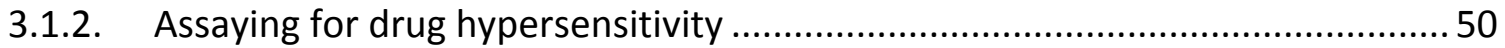

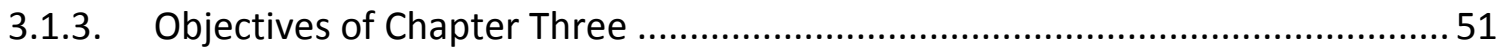

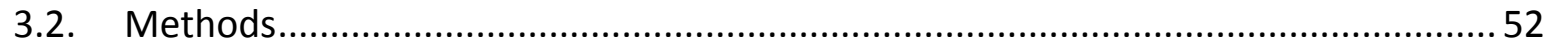

3.2.1. Estimation of unique mutants versus library size ......................................... 52

3.2.2. Construction of the transposon mutant collection.......................................... 53

3.2.3. Primary assay for drug hypersensitivity ........................................................ 54

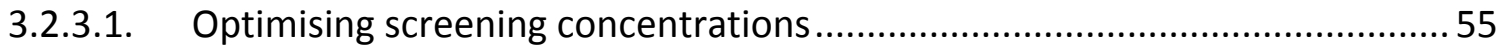

3.2.4. Secondary assay for drug hypersensitivity........................................................ 56

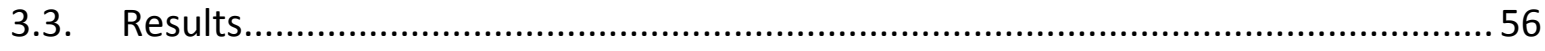

3.3.1. Drug screening concentration optimisation ................................................... 56

3.3.2. Growth of the treated-MRC10 control strain in LB...................................... 57

3.3.3. Control strains for quantitating drug hypersensitivity ................................... 57

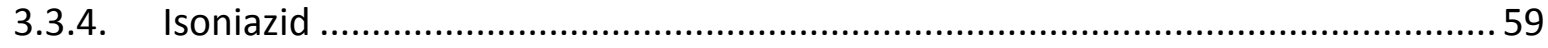

3.3.4.1. Primary assay for drug hypersensitivity ........................................................ 59

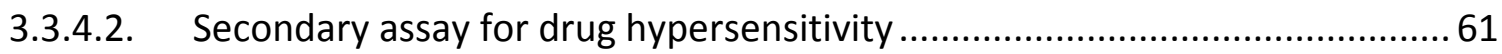

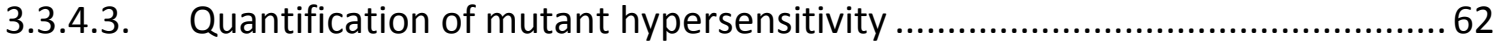

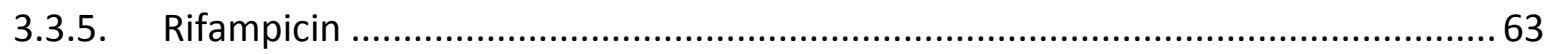

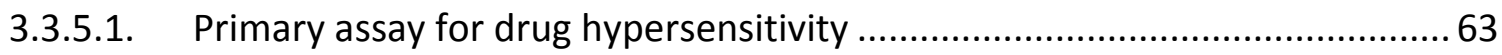

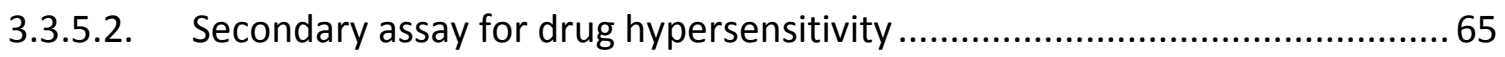

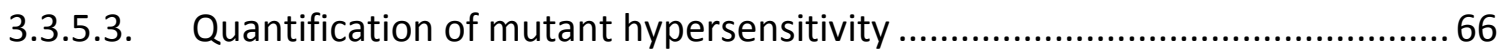


3.3.5.4. Growth curve of rifampicin hypersensitive mutant myco4005 ...................67 67

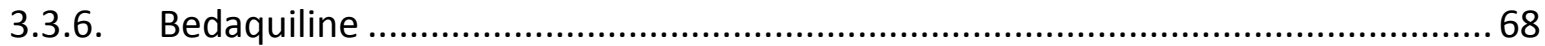

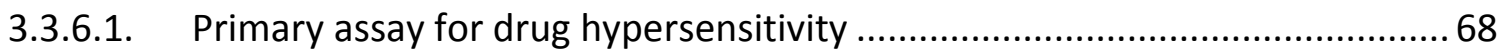

3.3.6.2. Secondary assay for drug hypersensitivity ................................................ 70

3.3.6.3. Quantification of mutant hypersensitivity ................................................ 71

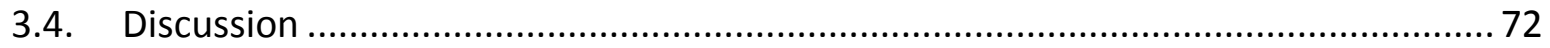

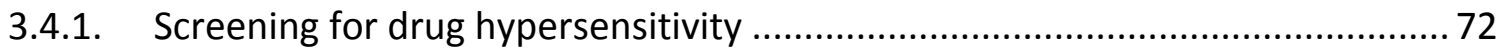

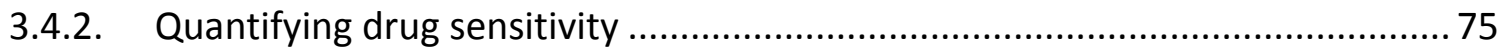

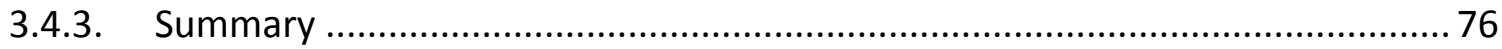

4. Genotypic validation of drug hypersensitive mutants of $M$. smegmatis ............77

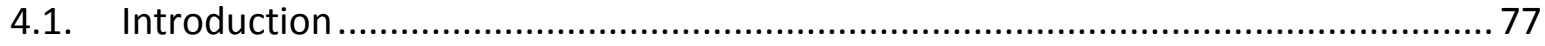

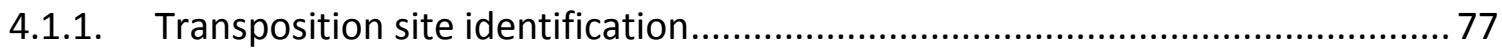

4.1.2. Conditional gene expression in mycobacteria ................................................ 78

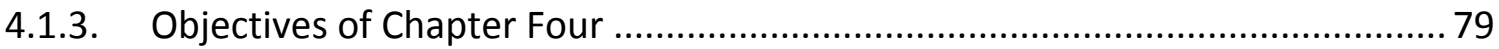

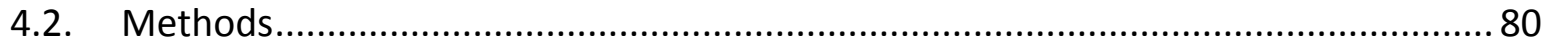

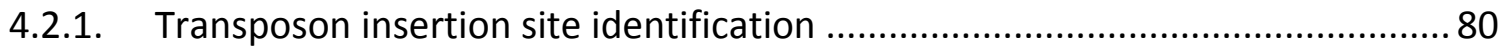

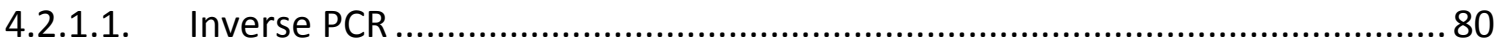

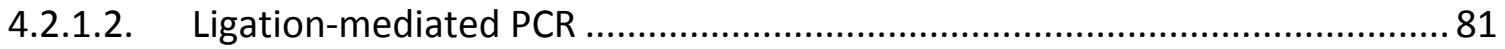

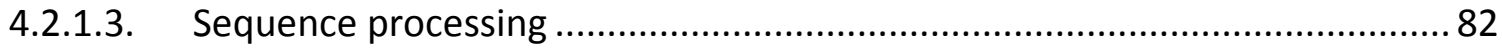

4.2.2. Optimisation of tetracycline concentration for induction of gene expression

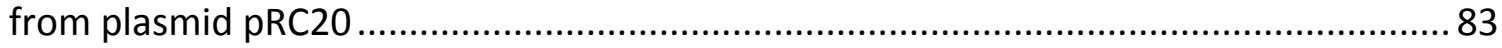

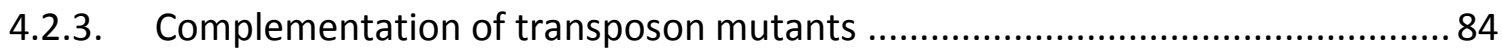

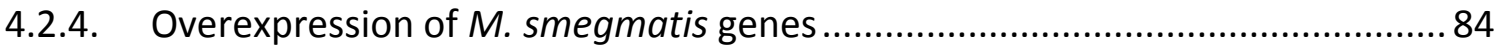

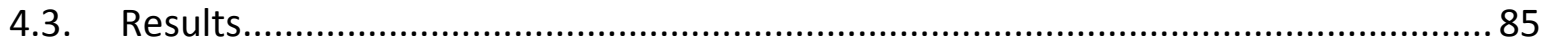

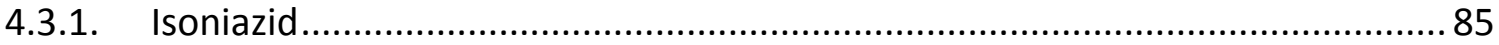

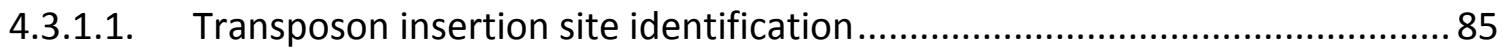

4.3.1.2. Complementation of isoniazid hypersensitive mutants............................... 87

4.3.1.3. Overexpression of genes related to isoniazid hypersensitivity...................... 90

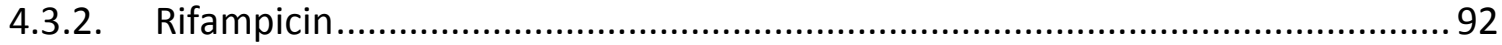

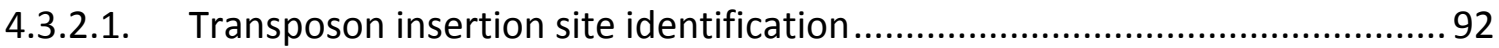

4.3.2.2. Complementation of rifampicin hypersensitive mutants ............................. 94

4.3.2.3. Overexpression of genes related to rifampicin hypersensitivity .................. 95

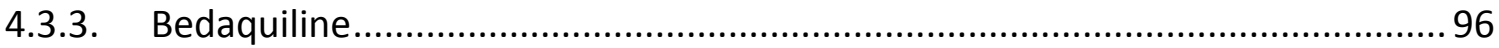

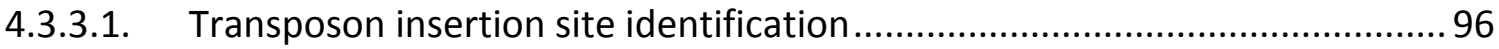




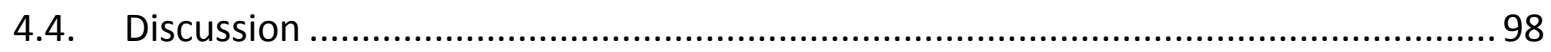

4.4.1. Identification of transposon insertion sites .................................................... 98

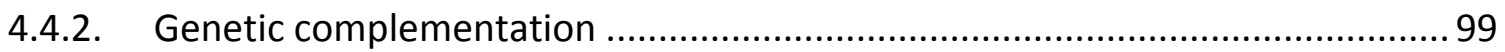

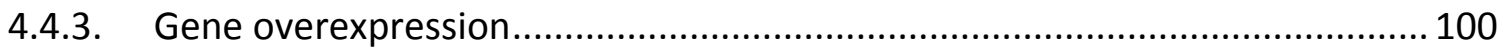

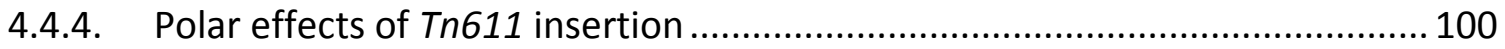

4.4.5. Genotypes related to isoniazid hypersensitivity........................................ 101

4.4.5.1. Links to known isoniazid mode of action and resistance mechanisms........ 101

4.4.5.2. Identification of potentially novel aspects of isoniazid's mode of action.... 103

4.4.6. Genotypes related to rifampicin hypersensitivity........................................ 106

4.4.6.1. Links to known rifampicin mode of action and resistance mechanisms...... 106

4.4.6.2. Identification of potentially novel aspects of rifampicin's mode of action . 107

4.4.7. Genotypes related to bedaquiline hypersensitivity ..................................... 109

4.4.7.1. Links to known bedaquiline mode of action and resistance mechanisms... 109

4.4.7.2. Identification of potentially novel aspects of bedaquiline's mode of action 110

4.4.8. Summary

5. Characterising drug sensitivity phenotypes of Tn611 mutants

.114

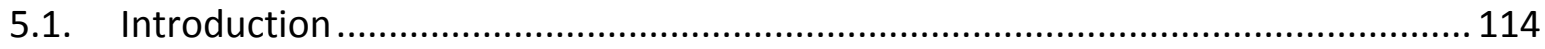

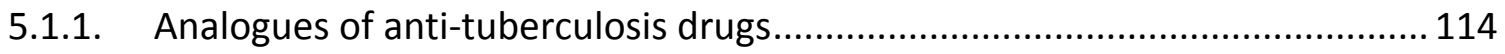

5.1.2. Isoniazid, rifampicin, and bedaquiline hypersensitive mutants ..................... 115

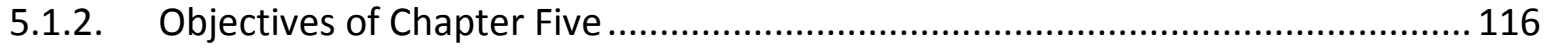

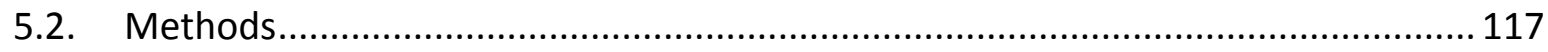

5.2.1. Cross sensitivity testing of transposon mutants to other drugs ...................... 117

5.2.2. Differential-sensitivity testing of transposon mutants to drug analogues...... 117

5.2.2.1. Selection of isoniazid analogues for differential-sensitivity testing............. 118

5.2.3. Overexpression strains versus isoniazid analogues ....................................... 118

5.2.3.1. katG overexpression strain versus isoniazid analogues ............................. 118

5.2.4. M. tuberculosis $\mathrm{H}_{37} \mathrm{Rv}$ versus isoniazid analogues ....................................... 119

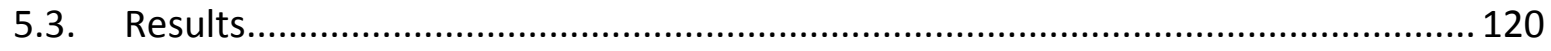

5.3.1. Cross-sensitivities of hypersensitive mutants............................................... 120

5.3.2. Differential-sensitivity of hypersensitive mutants ........................................ 120

5.3.2.1. Rifapentine versus rifampicin hypersensitive transposon mutants............. 120

5.3.2.2. Isoniazid analogues tested in isoniazid hypersensitive transposon mutants 121 
5.1.1. Isoniazid analogues versus overexpression strains.

5.1.2. Activity of isoniazid analogues in wild-type mycobacterial strains ................ 129

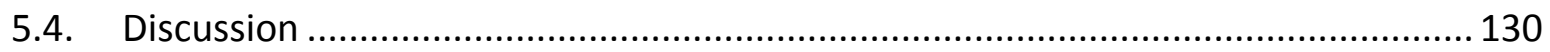

5.4.1. Cross-sensitivity of hypersensitive transposon mutants .............................. 130

5.4.2. Differential-sensitivities of hypersensitive transposon mutants to drug

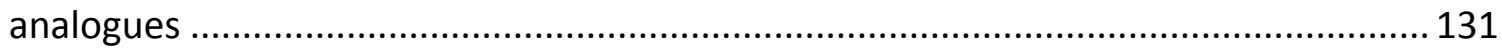

5.4.2.1. Role of KatG in activation of isoniazid analogues ..................................... 134

5.4.3. Activity of isoniazid analogues against M. tuberculosis ................................. 135

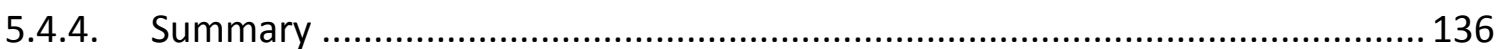

6. General Discussion

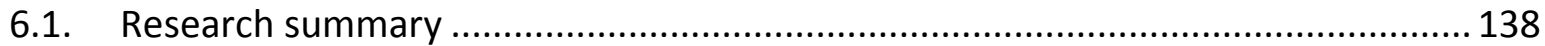

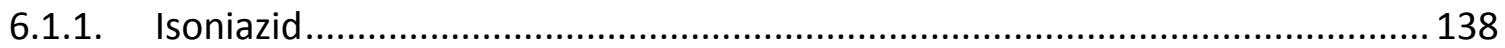

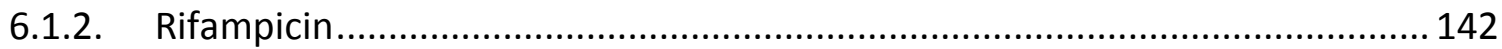

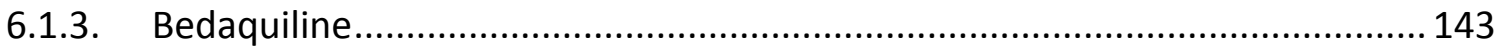

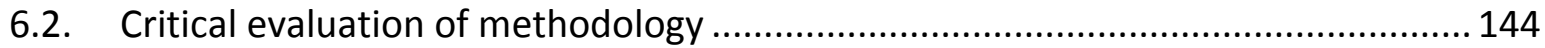

6.2.1. Limitation of using M. smegmatis $\mathrm{mc}^{2} 155$ as the genetic model................... 144

6.2.2. Use of Tn611 for transposon mutagenesis ................................................. 145

6.1.1. Transposon mutant collection coverage...................................................... 146

6.1.2. Quantifying mutant hypersensitivity ........................................................ 148

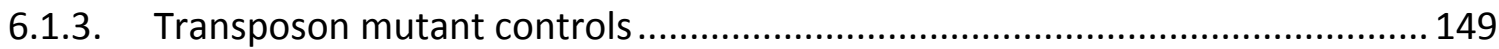

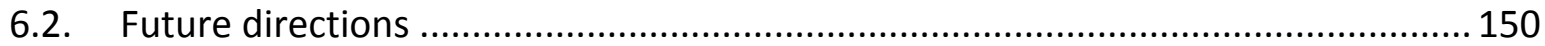

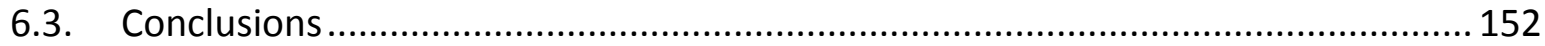

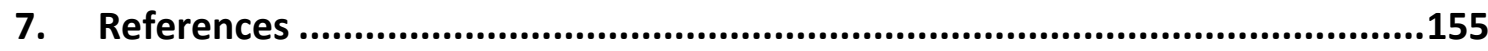

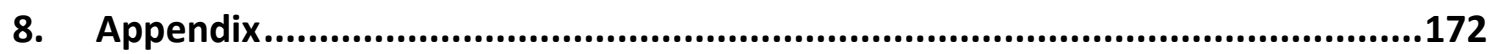

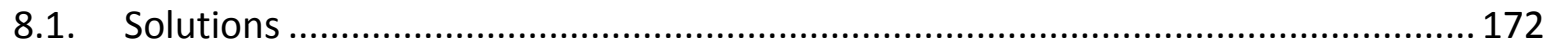

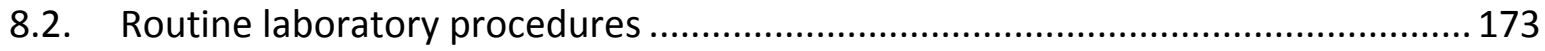

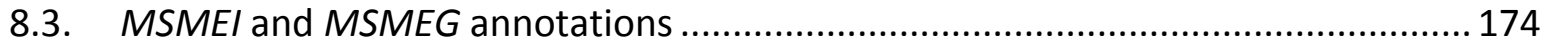

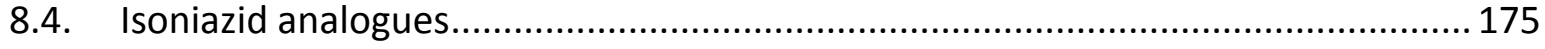

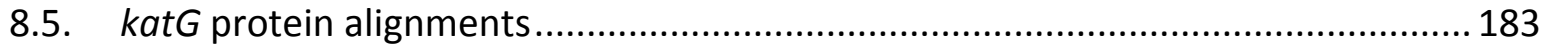

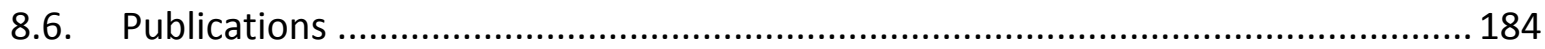




\section{List of Figures}

Figure 1.1: Estimated global tuberculosis incidence rates for 2013.................................... 2

Figure 1.2: Estimated number of global multi-drug resistant tuberculosis cases for $2013 \ldots \ldots . .3$

Figure 1.3: Ziehl-Neelsen-stained $M$. smegmatis............................................................. 7

Figure 1.4: Structure of the mycobacterial cell wall. ........................................................... 8

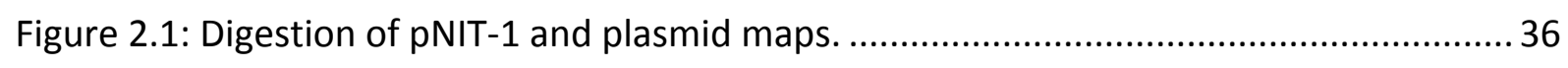

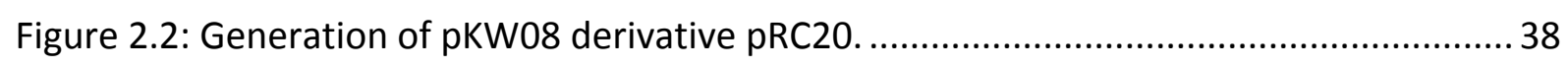

Figure 2.3: Layout of transposon mutant collection plates. ............................................... 40

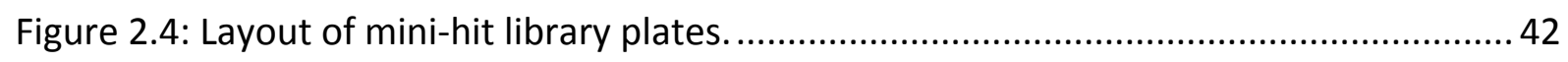

Figure 2.5: Plate setup for primary assays for drug hypersensitivity. .................................. 43

Figure 2.6: Plate setup for secondary assays for drug hypersensitivity................................ 44

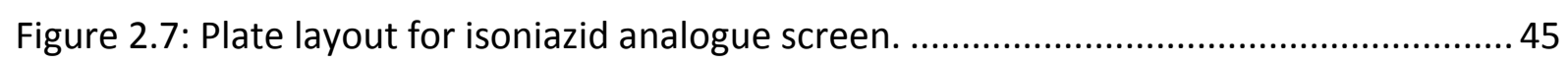

Figure 2.8: Plate layout for dose-response assays. ........................................................... 46

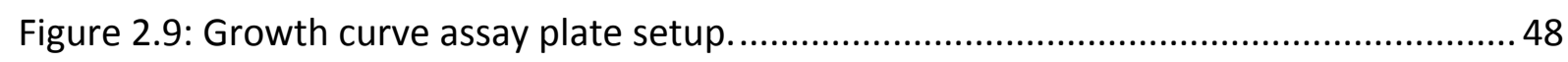

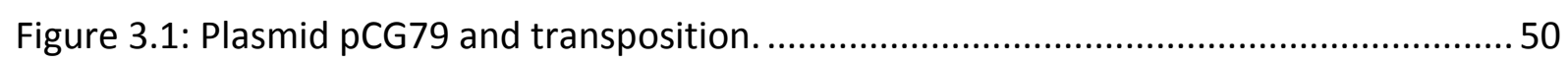

Figure 3.2: Number of unique mutants versus library size................................................ 53

Figure 3.3: Comparison of MIC of random mutant control strains. ...................................... 58

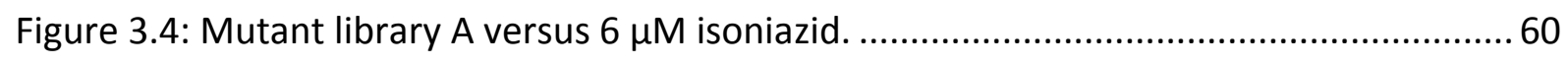

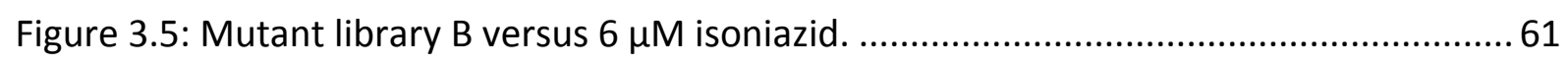

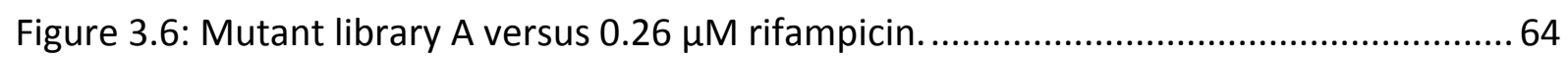

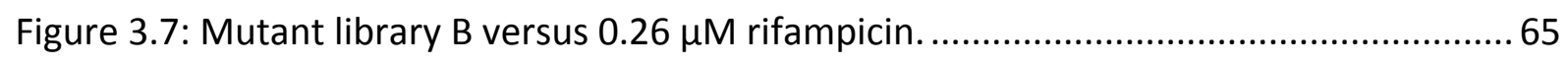

Figure 3.8: Growth curve of rifampicin hypersensitive strains........................................... 68

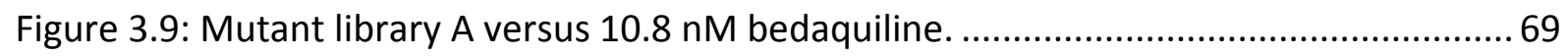

Figure 3.10: Mutant library B versus $10.8 \mathrm{nM}$ bedaquiline. ................................................ 70

Figure 4.1: Tetracycline inducible promoter of plasmid pKW08. ........................................ 79

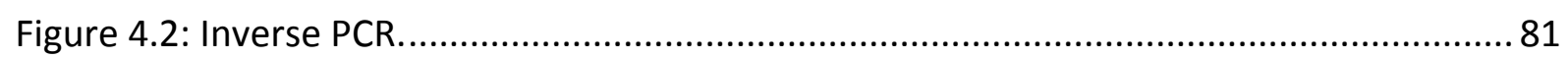

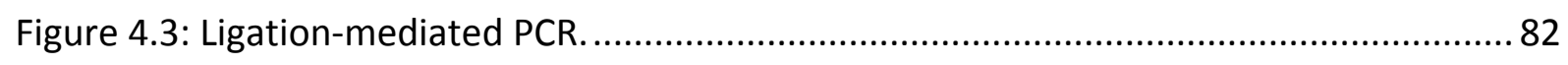

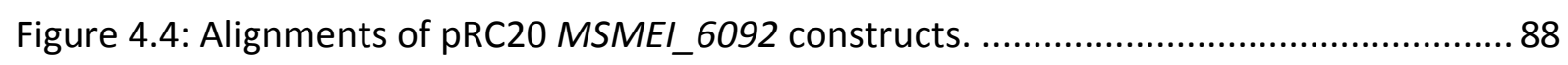

Figure 4.5: Optimisation of inducer concentration for complementation assays.................. 89

Figure 4.6: Complementation of isoniazid hypersensitive transposon mutants.................... 90

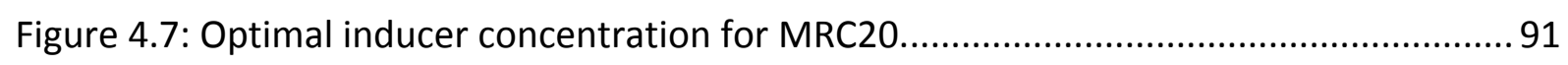


Figure 4.8: Dose response curve of MRC20 versus isoniazid \pm inducer. 91

Figure 4.9: Overexpression strains versus isoniazid. 92

Figure 4.10: Dose response curve of MRC20 versus rifampicin \pm inducer. 95

Figure 4.11: Overexpression strains versus rifampicin. .... .96

Figure 5.1: Structure of isoniazid analogues active against $M$. smegmatis $\mathrm{mc}^{2} 155$ 122

Figure 5.2: Growth-inhibition of WT M. smegmatis $\mathrm{mc}^{2} 155$ by isoniazid analogues. 123

Figure 5.3: Overexpression strains tested with isoniazid analogues. 127

Figure 5.4: $k a t G_{s m}$ overexpression strain versus isoniazid. 128

Figure 5.5: $\mathrm{katG}_{s m}$ overexpression strain versus isoniazid analogues. 129 


\section{List of Tables}

Table 1.1: Modes of action of current anti-tuberculosis drugs. .......................................... 12

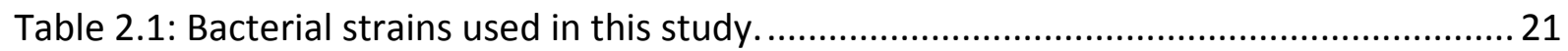

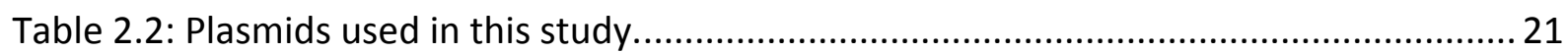

Table 2.3: Antibiotic concentrations used for marker selection......................................... 23

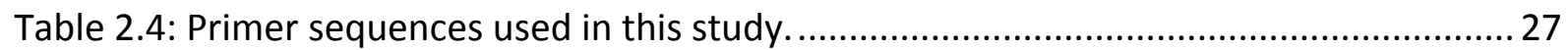

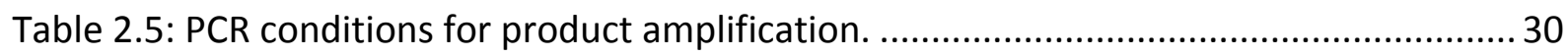

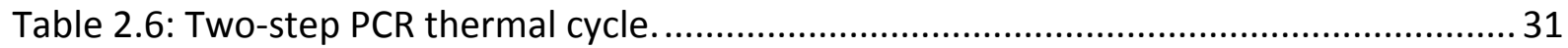

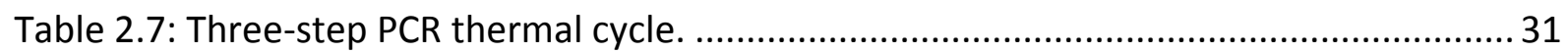

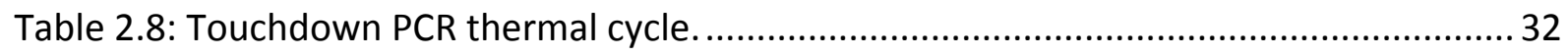

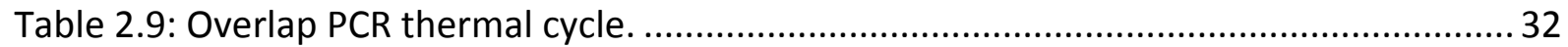

Table 3.1: MIC values for anti-TB drugs versus MRC10 and optimised screening

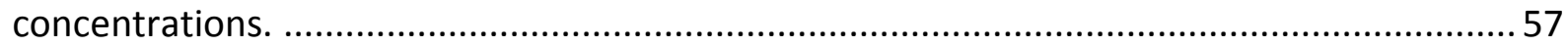

Table 3.2: Growth-inhibition of top secondary assay hits versus isoniazid...........................62 62

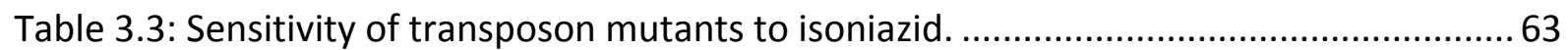

Table 3.4: Growth-inhibition of top secondary assay hits versus rifampicin..........................66

Table 3.5: Sensitivity of transposon mutants to rifampicin............................................... 67

Table 3.6: Growth-inhibition percentage of top secondary assay hits versus bedaquiline..... 71

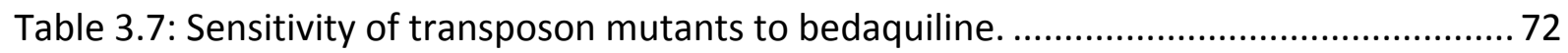

Table 4.1: Transposon insertion sites in isoniazid hypersensitive mutants............................. 86

Table 4.2: Protein similarity matrix of Fur proteins from M. smegmatis $\mathrm{mc}^{2} 155$ and

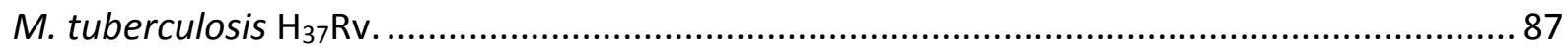

Table 4.3: Transposon insertion sites in rifampicin hypersensitive mutants. ....................... 93

Table 4.4: Transposon insertion site for bedaquiline hypersensitive mutants. ......................97

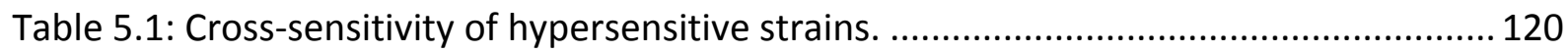

Table 5.2: Sensitivity of rifampicin hypersensitive mutants to rifapentine......................... 121

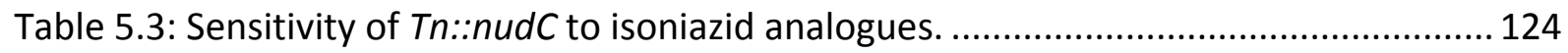

Table 5.4: MICs of isoniazid analogues for WT M. smegmatis and M. tuberculosis.............. 130 


\section{List of Abbreviations}

\begin{tabular}{|c|c|}
\hline AIDS & Acquired immune deficiency syndrome \\
\hline ATP & Adenine triphosphate \\
\hline BLAST & Basic Local Alignment Search Tool \\
\hline bp & Base pair \\
\hline CFU & Colony forming unit \\
\hline BCG & Bacillus Calmette-Guérin \\
\hline $\mathrm{ddH}_{2} \mathrm{O}$ & Distilled deionised water \\
\hline DMSO & Dimethyl sulfoxide \\
\hline DNA & Deoxyribonucleic acid \\
\hline DTP & Developmental therapeutics program \\
\hline E. coli & Escherichia coli \\
\hline EDTA & Ethylenediaminetetraacetic acid \\
\hline $\mathrm{F}_{1}$ & Factor of inhibition \\
\hline gDNA & Genomic DNA \\
\hline GFP & Green fluorescent protein \\
\hline HIV & Human immunodeficiency virus \\
\hline Hyg & Hygromycin B \\
\hline $\mathrm{Hyg}^{\mathrm{R}}$ & Hygromycin resistance \\
\hline INH & Isoniazid \\
\hline Kan & Kanamycin \\
\hline $\operatorname{Kan}^{R}$ & Kanamycin resistance \\
\hline $\mathrm{Kb}$ & Kilobase \\
\hline LA & LB agar \\
\hline LB & Luria broth \\
\hline MCS & Multiple cloning site \\
\hline MIC & Minimum inhibitory concentration \\
\hline MDR-TB & Multi-drug resistant tuberculosis \\
\hline M. africanum & Mycobacterium africanum \\
\hline M. bovis & Mycobacterium bovis \\
\hline M. canetti & Mycobacterium canetti \\
\hline M. microti & Mycobacterium microti \\
\hline M. orygis & Mycobacterium orygis \\
\hline M. pinnipedi & Mycobacterium pippinedi \\
\hline M. smegmatis & Mycobacterium smegmatis \\
\hline M. tuberculosis & Mycobacterium tuberculosis \\
\hline mRNA & Messenger RNA \\
\hline NIH & National Institute of Health \\
\hline NAD & Nicotinamide adenine dinucleotide \\
\hline $\mathrm{NADH}$ & Nicotinamide adenine dinucleotide hydride \\
\hline NZ & New Zealand \\
\hline OADC & Oleic acid, albumin, dextrose, catalase \\
\hline $\mathrm{OD}_{600}$ & Optical density at $600 \mathrm{~nm}$ \\
\hline ORF & Open reading frame \\
\hline OriE & E. coli origin of replication \\
\hline OriM & Mycobacterial origin of replication \\
\hline
\end{tabular}




$\begin{array}{ll}\text { PCR } & \text { Polymerase chain reaction } \\ \text { RD1 } & \text { Region of difference 1 } \\ \text { RFU } & \text { Relative fluorescence unit } \\ \text { RNA } & \text { Ribonucleic acid } \\ \text { rRNA } & \text { Ribosomal ribonucleic acid } \\ \text { Spec }^{R} & \text { Spectinomycin resistance } \\ \text { Strep }^{R} & \text { Streptomycin resistance } \\ \text { TAE } & \text { Tris acetate-EDTA } \\ \text { TB } & \text { Tuberculosis } \\ \text { TC } & \text { Tetracycline } \\ \text { TE } & \text { Tris-EDTA } \\ \text { ts } & \text { Temperature sensitive } \\ \text { V/v } & \text { Volume/volume } \\ \text { VUW } & \text { Victoria University of Wellington } \\ \text { WHO } & \text { World health organisation } \\ \text { WT } & \text { Wild-type } \\ \text { W/v } & \text { Weight/volume } \\ \text { XDR-TB } & \text { Extensively-drug resistant tuberculosis }\end{array}$




\section{Publications}

Campen, R.L., Ackerley, D.F., Cook, G.M., and O'Toole, R.F. (2015). Development of a Mycobacterium smegmatis transposon mutant array for characterising the mechanism of action of tuberculosis drugs: Findings with isoniazid and its structural analogues. Tuberculosis Article In Press. 


\section{General Introduction}

\subsection{Tuberculosis}

TB is a disease primarily caused by Mycobacterium tuberculosis, and is the first equal cause of mortality by a single pathogen along with the human immunodeficiency virus / acquired immune deficiency syndrome (HIV/AIDS) (WHO, 2014). The incidence of TB is worst amongst the poor due to factors such as inadequate health care, overcrowding and malnutrition (Bucher et al., 1999; Marais et al., 2013). As such, improvements in social economic conditions have been shown to improve TB control (Stop TB Partnership, 2006). Other significant risk factors for TB include substance abuse, smoking, chronic lung disease, and the presence of co-morbidities such as HIV and diabetes (Marais et al., 2013). These risk factors either compromise the immune system or lung integrity, increasing the rate of developing active disease, and often affecting treatment outcomes. It is estimated that one third of the world's population is infected with the etiological agent of TB, M. tuberculosis (Dye et al., 1999). Susceptibility to TB varies with age, with high rates in young children, lower rates in older children, and higher rates again after adolescence (Dubos and Dubos, 1952). M. tuberculosis can be divided into six lineages based upon genetic polymorphisms, with the different lineages varying in terms of geographic distribution, virulence, and drug resistance (Click et al., 2012; Gagneux and Small, 2007; Gagneux et al., 2006; Parwati et al., 2010; Reed et al., 2009).

In 2013 there were an estimated 9 million new cases of TB in the world, and 1.5 million deaths (WHO, 2014). Of the 9 million new cases, approximately 56\% of cases occurred in South-East Asia and the Western Pacific region, with China accounting for $11 \%$ and India $24 \%$ of cases (Figure 1.1). A further $25 \%$ of all new cases were from Africa, which had the highest incidence rate along with the highest mortality rate of any region. 

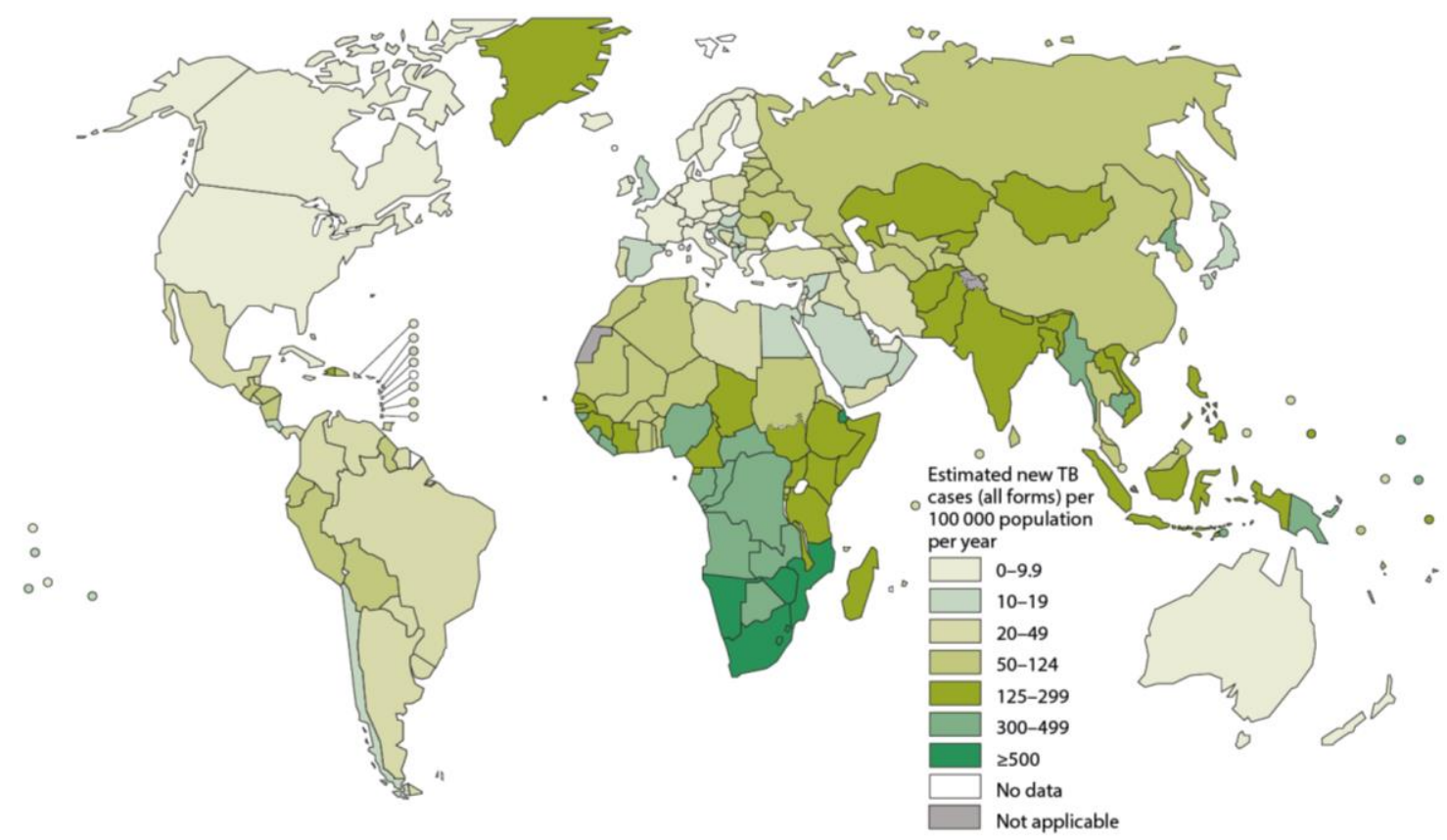

Figure 1.1: Estimated global tuberculosis incidence rates for 2013. Image reproduced from the Global tuberculosis report 2014 with permission (WHO, 2014).

Resistance to anti-TB drugs is widespread with drug resistant cases reported in all countries surveyed by the World Health Organisation (WHO) (Figure 1.2). Multi-drug resistant TB (MDR-TB), defined as resistant to at least the two front line TB drugs isoniazid and rifampicin, is of significant clinical importance as it has an associated lower cure rate $(48 \%$ globally in 2011) and higher mortality rate. The incidence of new cases of MDR-TB globally was around 3.5\% in 2013 (480,000); however, in some Asian and Eastern European counties the incidence was as high as 35\% for new cases, and $75 \%$ for previously treated cases. Combined, India, China, and the Russian Federation account for over $50 \%$ of all MDR-TB cases. Approximately 9\% of MDR-TB cases in 2013 were extensively drug resistant (XDR-TB), which displays additional resistances to fluoroquinolone and a second-line injectable agent. XDR-TB has a much lower cure rate $(22 \%$ in 2011$)$ and has now been detected in over 100 countries, with several cases of totally drug resistant XDR-TB reported (Udwadia et al., 2012; WHO, 2014). 

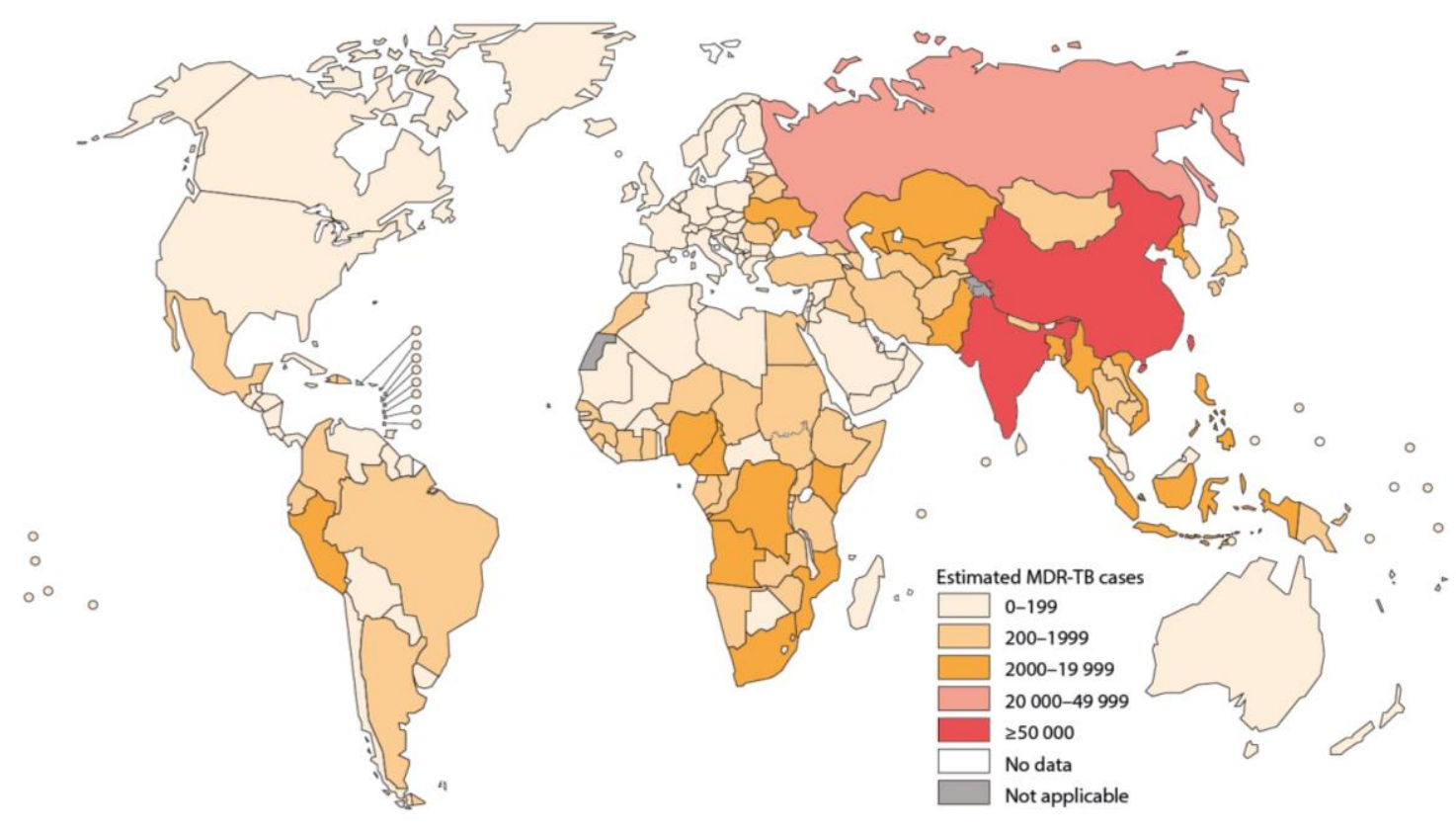

Figure 1.2: Estimated number of global multi-drug resistant tuberculosis cases for 2013. Image reproduced from the Global tuberculosis report 2014 with permission (WHO, 2014).

In the 1980's TB was thought to largely be under control but by the 1990's the HIV pandemic was driving a resurgence of $M$. tuberculosis infection (Murray et al., 1990). This was particularly true in sub-Saharan Africa where HIV/AIDS was becoming a leading cause of morbidity and mortality. HIV infection is associated with increased susceptibility to TB due to the immune-compromising effects of the HIV virus (Hopewell, 1992). HIV co-infection increases the reactivation risk of TB from a 5-10\% lifetime risk to a $10 \%$ risk per year (Shenoi and Friedland, 2009; Suthar et al., 2012). HIV co-infection also shows a significant association with TB drug resistance, including MDR-TB and XDR-TB (Shenoi and Friedland, 2009). M. tuberculosis infection in HIV positive cases represents over $10 \%$ of total cases annually, of which $80 \%$ occur in Africa, with TB responsible for $25 \%$ of deaths in HIV positive individuals (WHO, 2014). A major obstacle in treating HIV/TB co-infected individuals is that the front line TB drug rifampicin activates the cytochrome p450 system that metabolises common anti-retroviral drugs used to treat HIV, thereby reducing their efficacy (L'homme et al., 2009; Niemi et al., 2003). Diabetes is another significant comorbidity for TB, with diabetics three times more likely to develop active TB, primarily due to a defective host immune response (Lönnroth et al., 2014). Additionally, treatment of TB is less effective in diabetics, resulting in lower cure rates and higher mortality (Baker et al., 2011). In 2013, 15\% of all adult cases of TB were associated with diabetes. The rates of diabetes in those countries most at risk to TB 
has increased over the past decade and is proposed to be in part responsible for the lower than expected reduction in the number of cases of TB in these regions during that time frame.

\subsubsection{Tuberculosis in New Zealand}

The incidence rate for TB in New Zealand (NZ) is 6.6 cases per 100,000 with a total of 279 new cases in 2012 (Lim and Heffernan, 2013). The majority of these cases (75\%) occurred in individuals born outside of NZ, indicating that place of origin is a significant risk factor for TB in NZ. There were four cases of MDR-TB in 2012 in NZ and a total of 32 cases of MDR-TB over the previous 10 years. Of the cases of MDR-TB in NZ only two out of the 32 were NZ born, and it is assumed the other 30 were infected with MDR-TB outside of NZ (Lim and Heffernan, 2013). To date there has only been a single case of XDR-TB, which occurred during 2010. The predominant TB lineage in NZ cases is lineage four (Euro-American), followed by lineages one (Indo-Oceanic), two (East Asian), and three (East African Indian) (Yen et al., 2013). NZ and Pacific Island born patients were predominantly lineage four; whereas, individuals born outside of NZ predominately had lineages common to their place of origin.

\subsection{Pathogenesis}

M. tuberculosis is an obligate pathogen for which humans are the primary host (Saviola and Bishai, 2006). Unlike other infectious mycobacteria such as M. bovis, that routinely causes disease in a large number of mammals (including humans); M. tuberculosis does not have any significant environmental reservoirs. Disease caused by $M$. tuberculosis most commonly manifests as a pulmonary infection, with $70 \%$ of TB cases occurring in the lungs (Harisinghani et al., 2000; Young et al., 2008). Extra-pulmonary TB will occur in $10-42 \%$ of patients, dependent on additional factors such as age, ethnicity, additional underlying disease, specific M. tuberculosis strain, and host immune state (Caws et al., 2008). During HIV co-infection, extra-pulmonary disease can be more common than pulmonary, with little or no lung involvement, and is often diagnosed as another disease and only identified as TB upon autopsy (Mudenda et al., 2012; von Reyn et al., 2011). The characteristic symptoms of 
pulmonary TB include a chronic cough, production of sputum, haemoptysis, a loss of appetite, weight loss, and night sweats (Lawn and Zumla, 2011). Infection spreads by inhalation of bacteria-containing droplets expelled from infected individual's lungs. The number of bacteria required to start a new infection has previously been reported to be as few as 1-3 bacilli (Riley, 1957); however, the number required for a successful infection in humans may be significantly higher (Orme, 2014). Once inhaled, the bacilli enter the alveoli of the lungs where they are phagocytosed by resident alveolar macrophages (Clark-Curtiss and Haydel, 2003; Mehta et al., 1996; Saunders and Cooper, 2000). The macrophages are then activated by T cells, stimulating the formation of the acidified phagolysosome, killing the phagocytosed bacteria (Clark-Curtiss and Haydel, 2003). However, M. tuberculosis can live within host macrophage phagosomes by preventing their fusion with lysosomes and therefore their acidification (Brown et al., 1969; Mwandumba et al., 2004). The immune system's response to $M$. tuberculosis infection is to wall off the site of infection, forming a granuloma or 'tubercule' within which the tissue undergoes the caseous necrosis characteristic of TB (Saunders and Cooper, 2000).

In $10 \%$ of cases, acute disease will continue as the bacilli spread throughout the lung and later into other organ systems including the kidneys, liver, and brain (Caws et al., 2008). In the remaining $90 \%$ of cases the infection is either successfully cleared or enters a latent disease state within which the bacteria are believed to be in a dormant growth phase, possibly similar to the sporulation phase exhibited by other bacterial species (Bentrup and Russell, 2001; Parrish et al., 1998). Evidence, however, is mounting that M. tuberculosis continues to replicate, albeit at a reduced rate, within the host during chronic infection (Gill et al., 2009). Individuals with latent TB have a $10 \%$ lifetime reactivation risk, although this rate becomes much higher if the patient is immunocompromised (Girardi et al., 1999; Selwyn et al., 1989). For example, HIV-positive individuals are 37 times more likely to develop active TB than HIV negative individuals (WHO, 2010), with HIV co-infection increasing reactivation to a $10 \%$ per year risk (Shenoi and Friedland, 2009; Suthar et al., 2012). Additionally, individuals with diabetes also have a three-fold higher risk of developing active disease (Dye and Williams, 2010). 


\subsection{Mycobacteria}

Mycobacteria belong to the family Mycobacteriaceae within the order Actinomycetales and are characteristically acid fast, have a mycolic-acid rich cell wall, and a high GC genome content (61-71\%) (Pitulle et al., 1992; Shinnick and Good, 1994). Mycobacteria are commonly divided into two groups, the fast growing and the slow growing mycobacteria, whereby the slow growing mycobacteria are classified as those that take longer than seven days for colonies to appear on solid media; whereas, the colonies of the fast growing species appear in less than seven days (Pitulle et al., 1992; Shinnick and Good, 1994).

The slow growing mycobacteria include those belonging to the M. tuberculosis complex that consists of the bacterial species $M$. tuberculosis, M. bovis, M. africanum, M. microti, $M$. orygis, M. pinnipedi, and M. canetti (Pitulle et al., 1992; Shinnick and Good, 1994). Each member of the M. tuberculosis complex has a preferred host, but is capable of causing disease in other mammals (Smith et al., 2006). This is in part due to their very similar genetic makeup, sharing more than 99.9\% genetic identity (Fleischmann et al., 2002; Gutacker et al., 2002; Huard et al., 2006). Evidence suggests M. tuberculosis-like bacteria have existed for around three million years, evolving in Africa alongside humans (Galagan, 2014). The fast growing mycobacteria, of which approximately 40 species have been identified, are primarily saprophytic and can inhabit a wide variety of habitats in the environment (Saviola and Bishai, 2006; Shinnick and Good, 1994). It was thought for a long time that the fast growers were simply environmental bacteria; however, more species are now demonstrating their ability to cause disease as opportunistic pathogens (Howard and Byrd, 2000).

\subsubsection{Physiology}

Mycobacteria are aflagellate, irregular rod shaped bacilli of approximately $0.3-0.5 \mu \mathrm{m}$ in diameter with variable length (Figure 1.3)(Martinez et al., 1999; Shinnick and Good, 1994). Mycobacteria are often referred to as gram-positive; however, they have a structurally different cell wall from most gram-positive bacteria and are better defined as acid-fast, as determined by Ziehl-Neelsen staining (Figure 1.3). 


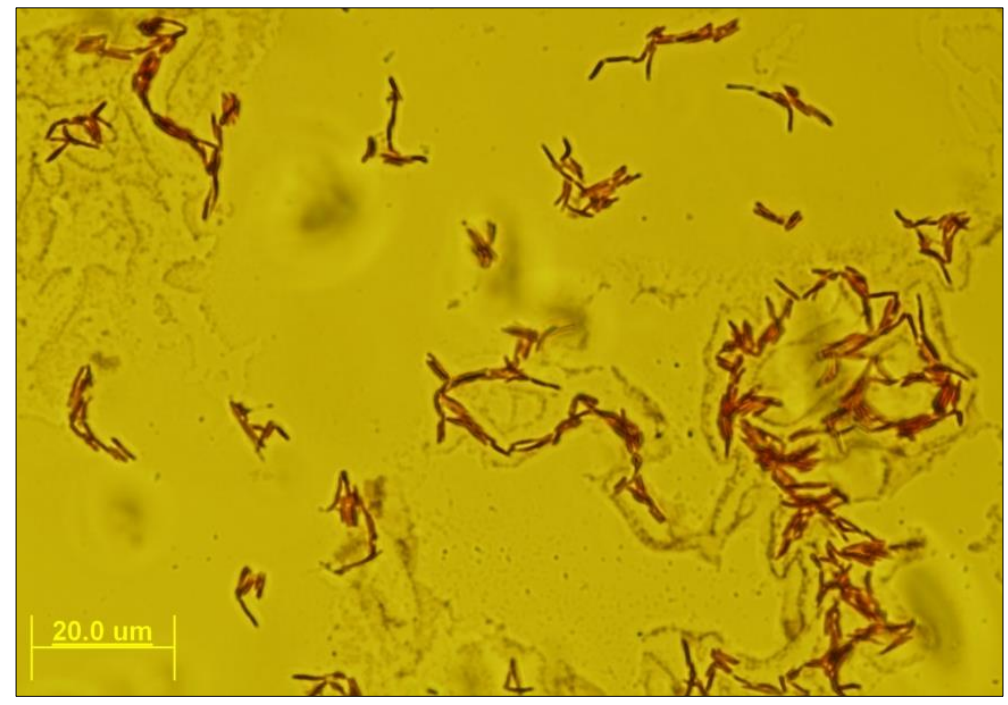

Figure 1.3: Ziehl-Neelsen-stained $M$. smegmatis. The lipid rich mycobacterial cell wall resists decolourisation during Zeihl-Neelsen staining; therefore, mycobacteria retain the red colour of the primary stain carbol fuchin. Non acid-fast bacteria lose the primary stain and show up blue from the counterstain methylene blue. Characteristic clumping of the mycobacteria can be observed in this image.

The cell wall of mycobacteria is composed of two segments: an inner segment, consisting of the peptidoglycan layer and the arabinogalactan layer with bound mycolic acids, and an outer segment that consists of free lipids (Figure 1.4)(Brennan, 2003). The free lipids in the outer segment along with the mycolic acids in the inner segment form an asymmetric lipid bilayer that gives mycobacteria their characteristic acid fastness. This outer lipid layer also contributes to the innate drug resistance of mycobacteria by limiting drug permeability (Jarlier and Nikaido, 1994). 


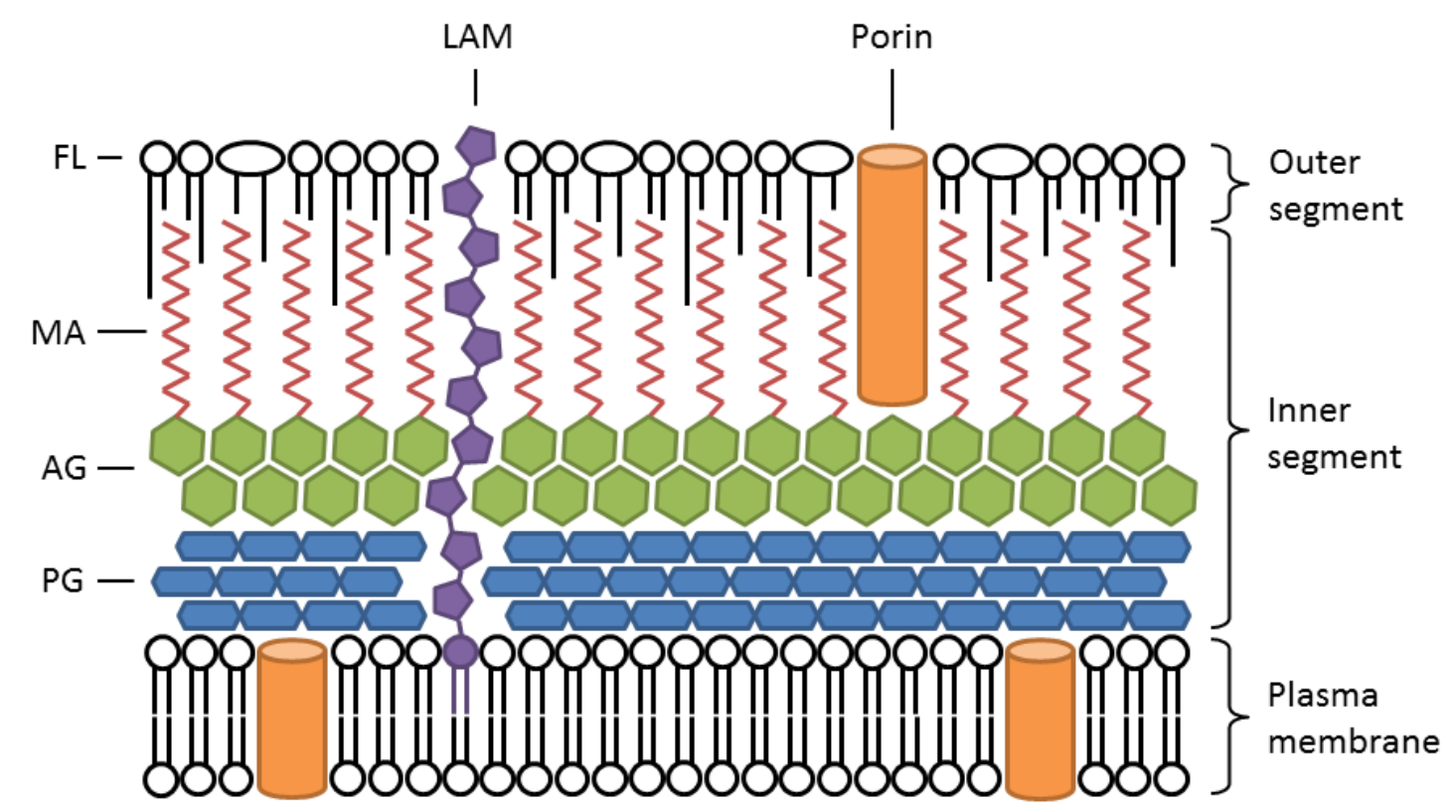

Figure 1.4: Structure of the mycobacterial cell wall. Working outwards, the mycobacterial envelope consists of the plasma membrane, a peptidoglycan (PG) layer, an arabinogalactan (AG) layer, a mycolic acid (MA) layer covalently bound to the arabinogalactan layer, and an outer segment consisting of free lipids (FL). The free lipids include phosphatidylinositol mannoses, dimycolytrehalose, phthiocerol dimycocerosate, and sulfolipids. The cell wall also contains lipoarabinomannas (LAM) that span the double-layered membrane, and various porins and cell wall proteins.

\subsubsection{Mycobacterium tuberculosis}

TB was first identified as a contagious disease in 1868 when it was shown to be transmitted from humans to rabbits (Saviola and Bishai, 2006), although it wasn't until 1882 that Robert Koch first directly observed the bacilli of $M$. tuberculosis responsible for the disease (Adler and Rose, 1996). M. tuberculosis belongs to the M. tuberculosis complex and is therefore a slow growing mycobacterium, with a doubling time of approximately 24 hours (Shinnick and Good, 1994). The M. tuberculosis genome encodes approximately 4000 genes. The genome of the common laboratory strain $\mathrm{H}_{37} \mathrm{Rv}$ was first sequenced in 1998 (Cole et al., 1998). As M. tuberculosis is an airborne pathogen, its handling requires specific safety precautions (Alderton and Smith, 2001), and as not all laboratories are equipped to handle virulent M. tuberculosis, surrogate strains are often used instead, including the attenuated $\mathrm{H}_{37} \mathrm{Ra}$ strain, $M$. bovis Bacillus Calmette-Guérin (BCG), or the low virulence environmental 
mycobacterium M. smegmatis (Altaf et al., 2010; Chaturvedi et al., 2007; Steenken et al., 1934).

\subsubsection{Mycobacterium smegmatis}

M. smegmatis is a soil dwelling saprophyte with a doubling time of approximately four hours, taking around three days for visible colonies to form on rich solid media (Shiloh and DiGiuseppe Champion, 2010). The fast doubling time of $M$. smegmatis makes it a useful surrogate for $M$. tuberculosis in high-throughput drug screening and large scale genetic based assays. Genetic studies of $M$. smegmatis were greatly facilitated by the discovery of a transformation efficient mutant, $m c^{2} 155$, that has a $10-100,000$ increase in efficiency (Snapper et al., 1990). Interestingly, M. smegmatis $\mathrm{mc}^{2} 155$ contains a genomic duplication of approximately 56 kilobases $(\mathrm{Kb})$ not present in the parental strain (Wang et al., 2008). This duplication contains the alcohol dehydrogenase gene $a d h C$, a gene previously predicted to be duplicated in the M. smegmatis $\mathrm{mc}^{2} 155$ geneome (Galamba et al., 2001). The gene encoding the $\beta$ subunit of the $F_{1}$ domain of the adenosine triphosphate (ATP) synthase, $a t p D$, is also predicted to be duplicated in the genome of $M$. smegmatis $\mathrm{mc}^{2} 155$ (Tran and Cook, 2005). However, atpD does not appear to be duplicated in the genome sequence. This suggests there may be more regions of the genome of $M$. smegmatis that are duplicated other than the characterised $56 \mathrm{~Kb}$ region. The original $M$. smegmatis reference genome sequence (NCBI Accession CP000480) had 6947 predicted genes, based mostly on homology to other known gene sequences. More recently, efforts to improve the accuracy of M. smegmatis $\mathrm{mc}^{2} 155$ 's genome annotations led to a newer updated genome sequence (NCBI Accession CP001663). Confirmation of gene sequences with protein sequence data led to refinement of the $M$. smegmatis $\mathrm{mc}^{2} 155$ genome, and this new reference sequence encodes only 6752 genes (Deshayes et al., 2007; Gallien et al., 2009; Perrodou et al., 2006).

Significant genetic differences exist between $M$. smegmatis and $M$. tuberculosis, whereby approximately half of the $M$. smegmatis genome contains genes that do not have homologues in M. tuberculosis (Altaf et al., 2010). This translates into phenotypic differences including different sensitivities to various compounds. However, M. smegmatis has a demonstrated ability to identify growth-inhibitory compounds that are active against 
M. tuberculosis in high-throughput assays (Altaf et al., 2010). Although the number of false positives are higher than when using the closer biological model $M$. bovis BCG, the majority of compounds active against $M$. tuberculosis can be identified using M. smegmatis. On balance, the benefits provided by the fast doubling time of $M$. smegmatis outweigh the disadvantages arising from genetic differences to M.tuberculosis. Any findings in M. smegmatis can ultimately be confirmed in lower throughput validation assays with M. tuberculosis.

\subsection{Treatment of tuberculosis}

Directly observed therapy, short course (DOTS) was a strategy developed to help control TB, encompassing policies around funding, case detection, treatment, logistics of drug supply, and monitoring (WHO, 2010). The Stop TB Strategy was developed as the successor to DOTS with the goal "to dramatically reduce the global burden of TB by 2015 in line with the Millennium Development Goals and the Stop TB Partnership targets" (Uplekar et al., 2006). Specifically, the WHO's Millennium Development Goals are to reverse the incidence of TB by 2015 (WHO, 2010); while, the Stop TB partnership has the additional goals of halving the mortality rates by 2015 compared to 1990 levels, and eliminating TB by 2050 (WHO, 2010).

Currently, treatment of drug-susceptible TB consists of a two-month course of isoniazid, rifampicin, pyrazinamide, and ethambutol, followed by a further four months of isoniazid and rifampicin alone (WHO, 2014). Treatment of drug-resistant TB requires the use of alternative second-line drugs that are generally more expensive, less effective, have higher associated toxicities, and lower cure rates. Treatment of drug-sensitive TB has a cure rate of over 90\%; while, the cure rate for MDR-TB is lower at only 50-70\% (WHO, 2010). Treatment of MDR-TB takes on average two years, while in extreme cases of XDR-TB there is no successful treatment. It is a commonly held belief that the current tools for the treatment and prevention of TB are ineffective, with a great need for drugs that can both reduce the treatment time and effectively treat drug-resistant TB, especially MDR-TB and XDR-TB (Duncan, 2003; O’Brien and Nunn, 2001; WHO, 2010; Young et al., 2008). 


\subsubsection{Anti-tuberculosis Drugs}

Prior to the advent of the first antibiotic, treatment of TB involved travelling to a sanatorium, where it was believed rest and a healthy diet would help to cure patients (Davis, 1996). Streptomycin, discovered in 1945, was the first antibiotic that was successful in treating TB infections (Pyle, 1947). Para-aminosalicylic acid was discovered almost simultaneously and was found to be effective for treating TB, especially in combination with streptomycin (Lienhardt et al., 2012). Isoniazid was discovered in 1951, demonstrating even greater activity against $M$. tuberculosis than either para-aminosalicylic acid or streptomycin and enabling the first three-drug treatment of TB (Pansy et al., 1952). By the mid-1980's, ethambutol and pyrazinamide had replaced para-aminosalicylic acid and streptomycin, and rifampicin had been introduced as well, giving the core first-line multidrug treatment for TB still used today. In cases of drug resistance, second-line drugs are utilised, including the aminoglycosides (including streptomycin), fluoroquinolones (e.g. ciprofloxacin), capreomycin, and the macrolides.

The current anti-TB drugs target a limited range of cellular processes, namely cell wall biosynthesis, DNA replication, transcription, translation, and folate biosynthesis (Table 1.1). Discovery of drugs that can inhibit novel targets is crucial for improving the current treatment regimens of TB, particularly drug-resistant TB (Duncan, 2003; O'Brien and Nunn, 2001; Young et al., 2008). 
Table 1.1: Modes of action of current anti-tuberculosis drugs.

\begin{tabular}{|c|c|c|}
\hline Drug & Mode of Action & References \\
\hline $\begin{array}{l}\text { Rifamycins } \\
\text { (including } \\
\text { rifampacin) }\end{array}$ & $\begin{array}{l}\text { Binds to the } \beta \text { Subunit of RNA polymerase, inhibiting } \\
\text { transcription }\end{array}$ & $\begin{array}{l}\text { (Wehrli et al., } \\
\text { 1968) }\end{array}$ \\
\hline Isoniazid & Inhibits mycolic acid biosynthesis & $\begin{array}{l}\text { (Banerjee et al., } \\
\text { 1994) }\end{array}$ \\
\hline Ethionamide & Inhibits mycolic acid biosynthesis & $\begin{array}{l}\text { (Banerjee et al., } \\
\text { 1994) }\end{array}$ \\
\hline Ethambutol & Inhibits arabinogalactan layer biosynthesis & $\begin{array}{l}\text { (Deng et al., } \\
\text { 1995) }\end{array}$ \\
\hline D-Cycloserine & $\begin{array}{l}\text { Prevents D-serine insertion into peptidoglycan, inhibiting } \\
\text { cell wall synthesis }\end{array}$ & $\begin{array}{l}\text { (Prosser and de } \\
\text { Carvalho, 2013) }\end{array}$ \\
\hline Fluoroquinolones & Inhibits DNA gyrase, inhibiting DNA synthesis & $\begin{array}{l}\text { (Fernandes, } \\
\text { 1988) }\end{array}$ \\
\hline Capreomycin & Interferes with the ribosome inhibiting protein synthesis & $\begin{array}{l}\text { (Maus et al., } \\
\text { 2005) }\end{array}$ \\
\hline Macrolides & Binds the 50 s ribosomal subunit, inhibiting translation & $\begin{array}{l}\text { (Chopra and } \\
\text { Brennan, 1998) }\end{array}$ \\
\hline P-aminosalicylic acid & $\begin{array}{l}\text { Inhibits dihydropteroate synthetase, inhibiting folate } \\
\text { biosynthesis }\end{array}$ & $\begin{array}{l}\text { (Chakraborty et } \\
\text { al., 2013) }\end{array}$ \\
\hline Aminoglycosides & Binds the 30 s ribosomal subunit, inhibiting translation & $\begin{array}{l}\text { (Moazed and } \\
\text { Noller, 1987) }\end{array}$ \\
\hline Pyrazinamide & Inhibits trans-translation & (Shi et al., 2011) \\
\hline
\end{tabular}

For 40 years after the discovery of rifampicin in the 1950s no new drug classes were developed for the treatment of TB. Research into anti-TB drugs has only become more active in the last decade. There are currently 10 drugs in the various phases of clinical trials, and a further nine in preclinical development (WHO, 2014). Of the 10 drugs currently in clinical trials, four have novel modes of action and are active against MDR-TB: Delamanid, PA-824, bedaquiline, and SQ109. Delamanid, previously known as OPC 67683, is a nitroimidazole that targets mycolic acid biosynthesis, and is similar to another recently discovered nitroimidazole PA-824 (Matsumoto et al., 2006; Singh et al., 2008; Stover et al., 2000). Delamanid is currently undergoing phase III clinical trials, while PA-824 is undergoing phase II clinical trials. Bedaquiline, previously known as TMC207, is a diarylquinoline, a novel class of anti-TB drug that targets the $C$ subunit of the ATP synthase $F_{0}$ domain. The two drugs, bedaquiline and delamanid, have been approved for use in treating restricted cases of MDR-TB (Villemagne et al., 2012). The only other anti-TB drug with a novel mode of action currently in clinical trials is SQ109, which is currently in phase II clinical trials. SQ109 inhibits integration of mycolic acid into the cell wall by inhibition of the transporter MmpL3, which 
appears to be involved in translocating mycolic acids to outside the cell (Protopopova et al., 2005; Tahlan et al., 2012).

\subsubsection{Drug resistance}

Clinical resistance has been reported for all of the drugs used to treat TB, with the first cases identified shortly after the initial use of streptomycin. Spontaneous drug resistance, in the clinical setting or in a laboratory environment, most often occurs from point mutations in the gene encoding the drug's target, reducing the affinity of the drug for its target. For example, mutations in the target of rifampicin, $r p o B$, led to rifampicin resistance (Ramaswamy and Musser, 1998), whereas mutations in the fluoroquinolone targets, the DNA gyrase encoding genes gyrA and gyrB, led to fluoroquinolone resistance (Kocagöz et al., 1996). Alternatively, if the drug is a prodrug, resistance often occurs from mutations in the prodrug activating enzyme, e.g. mutations in katG which encodes the catalase peroxidase enzyme KatG responsible for activating isoniazid lead to isoniazid resistance.

The long treatment time for TB (i.e. 6 months or longer) has been shown to play a significant role in the generation of drug resistance due to treatment non-compliance (Chao and Rubin, 2010; Claxton et al., 2001). This non-compliance selects for drug-resistant populations and has been demonstrated by Saunders et al. (2011) who sequenced serial isolates from a patient who was not adhering to the treatment regimen. Over the course of treatment the isolates developed sequential isoniazid then rifampicin resistance, with only two single nucleotide changes identified in the genome, one in $k a t G$ and one in $r p o B$.

\subsubsection{Modes of action of the anti-tuberculosis drugs to be examined in this study}

Isoniazid is an inhibitor of mycolic acid biosynthesis, an important substrate of the mycobacterial cell wall. Specifically, isoniazid inhibits the fatty acid synthase II (FASII) enoyl-acyl carrier protein reductase (InhA). Isoniazid was first shown to have anti-TB activity in 1952, and was far superior to any agents in use at the time (Bernstein et al., 1952; Pansy et al., 1952). Despite its long history of use, little was known about the mode of action of 
isoniazid until recently. Loss of catalase activity was long known to accompany isoniazid resistance in mycobacteria, but it was not until the advent of genetic techniques that mutations in the catalase/peroxidase katG were identified as the cause of both isoniazid resistance and catalase negative phenotypes (Zhang et al., 1992). InhA was first identified in the isoniazid mode of action when a catalase positive mutant resistant to isoniazid was identified carrying mutations in inhA (Banerjee et al., 1994). Subsequent studies have shown that isoniazid is a pro-drug that is activated by KatG into a reactive species that reacts with nicotinamide adenine dinucleotide (NAD) to form the isoniazid-NAD (INH-NAD) adduct responsible for inhibition of InhA (Johnsson and Schultz, 1994; Rozwarski et al., 1998; Wilming and Johnsson, 1999). Other targets have been suggested for isoniazid since, including ketoacyl synthase A (KasA) (Mdluli et al., 1998) and dihydrofolate reductase (DhfR) (Argyrou et al., 2006), although the lack of correlation between overexpression of these proteins and increased isoniazid resistance has helped support the hypothesis that InhA is the drug's primary target (Larsen et al., 2002; Wang et al., 2010). However, multiple isoniazid radicals have been demonstrated (Wengenack and Rusnak, 2001), as well as a range of secondary radicals (Ito et al., 1992; Timmins et al., 2004; Timperio et al., 2005; Van Zyl and Van Der Walt, 1994) whose activity within the mycobacterial cell supports the idea that the mode of action of isoniazid is broader than just inhibition of InhA.

Rifampicin is a transcriptional inhibitor which binds to, and inhibits the $\beta$ subunit of the DNA dependent RNA polymerase enzyme (RpoB) in bacteria (Wehrli et al., 1968), including mycobacteria (Levin and Hatfull, 1993). Rifampicin is a semi-synthetic drug derived from the natural product rifamycin (Maggi et al., 1966). This derivative was found to have improved activity against gram-negative bacteria and the actinobacteria including mycobacteria. Clinical resistance to rifampicin is almost entirely due to mutations in $r p o B$, with $96 \%$ of mutations in rifampicin-resistant clinical isolates occurring within an 81 base pair (bp) sequence of the gene (Ramaswamy and Musser, 1998).

Bedaquiline is a diarylquinoline, the first novel class of anti-mycobacterials to be discovered in 40 years (Andries et al., 2005). The primary target for bedaquiline is the $C$ subunit of the $F_{0}$ ATP synthase domain encoded by atpE (Andries et al., 2005; Koul et al., 2007). Due to its recent discovery, limited work has been done investigating the mode of action of bedaquiline beyond the primary ATP synthase target. 


\subsection{Drug Discovery}

The TB drug development pipeline involves identification of compounds that are bacteriostatic or bactericidal against $M$. tuberculosis, development of lead compounds including identification of drug modes of action, and finally progression into clinical trials (Duncan, 2003). These anti-TB compounds are identified from high-throughput screening of chemical libraries against mycobacteria (whole-cell screening), or from screening of isolated enzyme targets (Balganesh et al., 2008). Whole-cell screening has been the most successful approach to drug discovery as it simultaneously assesses all factors related to a drug's efficacy (Lechartier et al., 2014; Payne et al., 2007). Numerous variations on whole cell screening exist using a number of different model systems including macrophage infection, nutrient starvation, non-replicating, latent systems, and incorporating various different animal models. While these approaches have the potential to identify compounds that will be active in the clinic, care needs to be taken as each model makes an assumption about M. tuberculosis infection that can lead to misinterpretation of results (Miller et al., 2009; Pethe et al., 2010).

A disadvantage to whole-cell based screening for anti-mycobacterial compounds is that no information on the mode of action of the drug is generated. The absence of any target information from whole-cell screening prevents the rational design of structural modifications to improve drug activity without further investigation of the drug's mode of action (Koul et al., 2011). However, whole-cell screening remains an effective way to identify new anti-TB drugs, including those with new modes of action. Whole-cell screening was used to identify bedaquiline, delamanid, PA-824, and SQ109, (Lechartier et al., 2014).

Screening of isolated enzyme targets has been largely unsuccessful in identifying anti-TB compounds due to difficulty with translating good target inhibition in biochemical assays into good bactericidal or bacteriostatic activity against whole cells (loerger et al., 2013). This difficulty largely stems from an incomplete understanding of what makes an enzyme a good drug target. An alternative approach is target-based whole-cell screening, whereby target information is incorporated into the whole-cell screen (Abrahams et al., 2012; loerger et al., 2013; Nisa et al., 2010). A common strategy in this type of screen is the conditional repression of a gene of interest with a system such as the tetracycline inducer and repressor 
system (Ehrt et al., 2005; Williams et al., 2010). By reducing the levels of the target protein the cells become more sensitive to compounds that inhibit that protein, with specific inhibitors demonstrating differential activity between the conditional mutant and wild-type (WT). This approach has been used to find inhibitors of an enzyme essential for cell division (ParA) (Nisa et al., 2010), an enzyme involved in vitamin B5 biosynthesis (PanC) (Abrahams et al., 2012), and the malate synthase enzyme (Krieger et al., 2012).

\subsubsection{Mode of action identification}

Mode of action identification studies are essential during development of novel anti-TB drugs, generating information on the primary drug target, secondary off target effects, and mechanisms of drug resistance (Azad and Wright, 2012; Lechartier et al., 2014). Drugs are pleiotropic by nature, and determining the primary and secondary targets provides information on whether drug-drug interactions are likely to occur when the novel drug is used in combination with current drugs, a consideration important in the multi-drug treatment of TB (Ginsberg and Spigelman, 2007). Additionally, identification of the primary and secondary targets enables a rational approach to chemical modifications to drugs to improve their efficacy against both the primary and secondary drug targets (Balganesh et al., 2008; Campbell et al., 2001; loerger et al., 2013).

There are three commonly used genetic techniques to investigate the mode of action of a drug: a) identification of genes involved in drug resistance, b) identification of genes whose transcription is altered upon drug treatment, and c) identification of genes involved in drug hypersensitivity. These three different genetic approaches complement each other well, and utilising all of them, combined with biochemical data, gives the most information on drug mode of action. Past studies have focused on the identification of resistance inducing mutations, and altered transcription upon gene treatment. To the best of my knowledge, only two previous studies have examined drug sensitising mutations in mycobacteria, one study by Alexander et al. (2003) looking for rifampicin hypersensitive mutants, and another by Daugherty et al. (2011) looking for mutants hypersensitive to a ubiquitin derived peptide. Therefore, the study of sensitising mutations for current anti-TB drugs has the potential to generate novel information on drug mode of action. 


\subsubsection{Resistance inducing mutations}

The majority of primary targets that have been identified for the current anti-mycobacterial drugs were found by studying genes involved in drug resistance (Andries et al., 2005; Banerjee et al., 1994; Belanger et al., 1996). Resistance to most TB drugs occurs through point mutations in the gene encoding the primary target. Alternatively, resistance can occur due to mutations in genes that are functionally interacting with the drug target. Therefore, identification of point mutations in drug resistant mutants has facilitated target identification. This approach can also identify enzymes responsible for activating pro-drugs when the primary resistance mechanism is loss of pro drug activation (Lechartier et al., 2014). Transposon mutagenesis has been used in a similar manner, although this type of mutation typically causes loss of function and consequently is not useful for determination of the primary targets (Billman-Jacobe et al., 2006). Drug resistance studies have a very narrow focus, singling out individual genes that directly interact with the compound, such as the primary drug target, pro-drug activating enzymes, or enzymes responsible for drug detoxification.

\subsubsection{Altered transcription with drug treatment}

Investigation into which genes are up/down-regulated in response to drug treatment provides a broader picture of drug mode of action. Transcriptional analysis is useful for identifying pathways that are inhibited by a drug, including both the primary and secondary target pathways. Transcriptional analysis can be performed on a small scale, using quantitative polymerase chain reaction (PCR) to measure changes in messenger RNA (mRNA) expression levels of individual genes, or on a larger scale, using micro-arrays to measure changes in mRNA expression levels of large sets of genes, or even the whole transcriptome (Boshoff, 2004). Large scale transcriptional analysis is useful for grouping compounds with similar modes of actions in mycobacteria. For example, Boshoff et al. (2004) measured the transcriptional response of $M$. tuberculosis to a range of growth inhibitory conditions, including drug treatment, and clustered the transcriptional profiles for each condition. This approach was able to group conditions with similar modes of action, e.g. isoniazid and ethionamide clustered together along with thiolactomycin, three drugs that all inhibit 
components of the FASII machinery. More recently, transcriptional profiling identified the mode of action of the novel anti-TB drug PA-824 as involving both inhibition of mycolic acid biosynthesis and nitric oxide induced (NO) respiratory poisoning (Manjunatha et al., 2009).

A limitation of the transcriptional analysis approach is that while affected pathways are determined, the specific gene target cannot be directly identified. Additionally, this approach is only as strong as the size of the gene set against which each new drug is tested. Finally, transcriptional analysis gives an incomplete description of a drug's mode of action (Sassetti et al., 2001). For example, transcriptional analysis does not provide information on constitutively expressed genes, or genes that are post-transcriptionally regulated.

\subsubsection{Hypersensitivity inducing mutations}

Analysis of mutations that cause drug hypersensitivity provides information on genes that are conditionally essential to maintaining WT drug sensitivity. Similar to transcriptional studies, this approach yields information on which pathways are inhibited by the drug (primary and secondary target pathways), as opposed to the specific molecular target. This approach is routinely used in the budding yeast Saccharomyces cerevisiae to study drug mode of action where it is termed chemical genetics (Parsons et al., 2004, 2006). A viable loss of function mutation within a pathway targeted by the drug will sensitise the organism to the drug, thereby identifying that pathway as being involved in the drug's mode of action. This approach also identifies specific proteins responsible for maintaining basal drug resistance, i.e. drug detoxification mechanisms (Alexander et al., 2003). It is necessary to isolate mutants and assay them individually for increased drug-sensitivity as the mutations of interest produce a recessive phenotype. Therefore, a common limitation of this technique is a poor genomic coverage for the collection of mutants being studied. This is especially pertinent when using a mutagenesis approach such as random transposon mutagenesis which is unable to create mutations in essential genes (Griffin et al., 2011; Sassetti et al., 2001, 2003). Despite this limitation, random transposon mutagenesis is routinely used to generate collections of random mutants for phenotypic screening, including screening for drug hypersensitivity, because of the ease of identifying the site of insertion, and the 
consistent nature of the mutation (loss of function) (Alexander et al., 2003; Daugherty et al., 2011; Gutacker et al., 2002).

\subsection{Random transposon mutagenesis of $M$. smegmatis}

Assaying for drug hypersensitivity requires the prior construction of a collection of viable mutants. This collection should be randomly generated, with good genome coverage and minimal overlap. Insertional mutagenesis using transposons is favoured for the generation of random bacterial mutants due to the inherent labelling of the site of mutation with the transposon DNA, which facilitates the identification of the genetic basis for the mutant phenotype (Billman-Jacobe et al., 2006; Prod'hom et al., 1998; Sassetti et al., 2001). A transposon insertion into a gene leads to a loss of function of the gene product, which has the important implication that only genes not essential for the organism's survival can harbour a transposon insertion (Sassetti et al., 2001). As such, transposon mutant libraries will only contain those genes that are non-essential for survival in the conditions used. This characteristic has been utilised for determining the varying set of essential genes under a range of conditions, including in vitro in various media, intra-macrophage survival, in vivo mouse models, and under different drug treatment conditions (Alexander et al., 2003; Billman-Jacobe et al., 2006; Daugherty et al., 2011; McAdam et al., 2002; Rengarajan et al., 2005; Sassetti et al., 2001, 2003).

Two different delivery systems exist for transposon mutagenesis in mycobacteria: phage based, such as фMycoMarT7, which delivers the Himar1 transposon (Sassetti et al., 2001), or plasmid based systems such as pCG79, which delivers the Tn611 transposon (Guilhot et al., 1994). Transposon mutagenesis systems often use delivery vectors which are temperature sensitive for replication in mycobacteria, which allows the process of transfection or transformation to be separated from the process of transposition. Transposition is a relatively rare event, but appropriate selection techniques can improve the yield of mutants obtained (Bardarov et al., 1997). Transformed/transfected cells can be plated out at a non-permissive temperature for plasmid/phage replication and only successful transposition events will grow. These strains can then be stored individually for later study. 


\subsection{Aims of this thesis}

The aim of this thesis was to generate information on drug mode of action by identifying the genetic basis of drug hypersensitivity in mutants of $M$. smegmatis $m^{2} 155$. To the best of my knowledge, only two previous studies have investigated the mode of action of anti-TB compounds through studying drug hypersensitive mutants, one published after this present study had begun (Alexander et al., 2003; Daugherty et al., 2011). To enable the identification of drug hypersensitive mutants of $M$. smegmatis $m^{2} 155$ we also aimed to develop a high-throughput assay for drug hypersensitivity. M. smegmatis $\mathrm{mc}^{2} 155$ was selected as a model for M. tuberculosis in this study as it was able to be used in the physical containment 2 (PC2) laboratories available, and as it provided time advantages over the slow growing models. Transposon mutagenesis was selected for generating the mutants of $M$. smegmatis $\mathrm{mc}^{2} 155$ due to the reported consistent nature of the mutations, and the ease of identifying the site of the genetic lesion responsible for the hypersensitivity phenotype.

The front line TB drugs isoniazid and rifampicin were selected for this study as the known aspects of their modes of action would enable validation of our methodology. Bedaquiline, which has recently been approved for limited treatment of MDR-TB, was also included in this study as little is known about its mode of action other than the primary target. Due to the absence of prior studies of this nature, there was a large potential to identify novel aspects of the modes of action of these three anti-TB drugs. A better understanding of the modes of action of these drugs would enable rational modifications of the drugs to improve treatment of TB. To this end the following specific aims were addressed:

1. Identification of isoniazid, rifampicin, and bedaquiline hypersensitive transposon mutants of $M$. smegmatis $\mathrm{mc}^{2} 155$. To achieve this aim the following sub aims were addressed: Construction of a transposon mutant collection in $M$. smegmatis $\mathrm{mc}^{2} 155$, and development of a high-throughput assay for drug hypersensitivity.

2. Identification and validation of the genotypic basis for the observed drug hypersensitivity to generate information about each drug's mode of action.

3. Characterisation of drug sensitivity phenotypes, including cross-sensitivities and differential-sensitivities to other anti-TB drugs and drug analogues, respectively. 


\section{Materials and Methods}

\subsection{Bacterial strains}

Strains used in this study are listed in Table 2.1 along with the relevant genotypic and phenotypic properties. Stocks of all strains were stored at $-80^{\circ} \mathrm{C}$ in $20 \%(\mathrm{v} / \mathrm{v})$ glycerol.

Table 2.1: Bacterial strains used in this study.

\begin{tabular}{|c|c|c|}
\hline Strain & Genotype and/or phenotype & Reference \\
\hline & 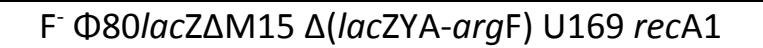 & \\
\hline E. coli $\mathrm{DH} 5 \alpha$ & $\begin{array}{c}\text { endA1 } h s d \mathrm{R} 17\left(\mathrm{r}_{\mathrm{k}}^{-}, \mathrm{m}_{\mathrm{k}}^{+}\right) \text {phoA supE44 thi-1 } \\
\text { gyrA96relA1 } \lambda^{-}\end{array}$ & Invitrogen ${ }^{\mathrm{TM}}$ \\
\hline $\begin{array}{l}\text { M. smegmatis } \\
\mathrm{mc}^{2} 155\end{array}$ & $\begin{array}{l}\text { High plasmid transformation efficiency strain of } \\
\text { M. smegmatis }\end{array}$ & (Snapper et al., 1990) \\
\hline $\begin{array}{l}\text { M. tuberculosis } \\
\qquad \mathrm{H}_{37} \mathrm{Rv}\end{array}$ & $\begin{array}{l}\text { Virulent laboratory reference strain of } \\
\text { M. tuberculosis }\end{array}$ & $\begin{array}{c}\text { (Oatway Jr. and } \\
\text { Steenken Jr., 1936; } \\
\text { Steenken et al., 1934) }\end{array}$ \\
\hline
\end{tabular}

\subsection{Plasmids}

Plasmids used in this study are listed in Table 2.2 along with the relevant properties.

Table 2.2: Plasmids used in this study.

\begin{tabular}{|c|c|c|}
\hline Plasmid & $\begin{array}{c}\text { Properties } \\
\end{array}$ & Reference \\
\hline pCG79 & $\begin{array}{c}\text { OriM (ts pAL5000), } \text { Kan }^{R} \text {, Strep } / \mathrm{Spec}^{\mathrm{R}}, \operatorname{Tn} 611 \\
\text { transposon }\end{array}$ & (Guilhot et al., 1994) \\
\hline pNIT-1 & $\begin{array}{c}\text { Mycobacterial conditional expression vector, OriE, } \\
\text { OriM, Kan }{ }^{R} \text {, NIT operon, MCS }\end{array}$ & (Pandey et al., 2009) \\
\hline pRC10 & Derivative of pNIT-1 with NIT operon removed & This study \\
\hline pLL192_hsp60 & OriE, OriM, Kan ${ }^{R}$, hsp60 driven GFP expression & $\begin{array}{l}\text { (Srivastava et al., } \\
\text { 2007) }\end{array}$ \\
\hline pKW08_LX & $\begin{array}{c}\text { Mycobacterial conditional expression vector, OriE, } \\
\text { OriM, } \mathrm{Hyg}^{\mathrm{R}} \text {, inducible } \mathrm{P}_{\mathrm{Tet}}, \mathrm{MCS}, \operatorname{lux} A B\end{array}$ & (Williams et al., 2010) \\
\hline pRC20 & $\begin{array}{c}\text { Derivative of pKW08_Lx with luxAB replaced with } \\
\text { GFP }\end{array}$ & This study \\
\hline
\end{tabular}

\subsection{Chemicals and reagents}

Unless otherwise specified all general reagents and chemicals were obtained from Sigma-Aldrich. HyperLadder ${ }^{\mathrm{TM}} 1 \mathrm{~Kb}$ DNA ladder and DNA loading dye were obtained from Bioline (Auckland, NZ). 


\subsection{Enzymes}

All restriction endonuclease enzymes were purchased from New England Biolabs (Auckland, NZ), as were Antarctic phosphatase, T4 DNA polymerase, and T4 polynucleotide kinase. The PCR enzyme master mixes BioMix ${ }^{\mathrm{TM}}$ Red and AccuSure ${ }^{\mathrm{TM}}$ were obtained from Bioline (Auckland, NZ). T4 DNA ligase, Proteinase $\mathrm{K}$ and lysozyme were obtained from Invitrogen. Phusion ${ }^{\circledR}$ polymerase was obtained from Thermo-Fisher Scientific.

\subsection{Growth media}

All media were made up in sterile distilled and deionised $\mathrm{H}_{2} \mathrm{O}\left(\mathrm{dd}_{2} \mathrm{O}\right)$ according to the manufacturer's instructions and sterilised by autoclaving at $121{ }^{\circ} \mathrm{C}$ for 30 minutes, unless stated otherwise.

\section{Luria Broth}

All Luria Broth (LB) and LB agar (LA) low salt powders (Duchefa Biochemie) were purchased from Total Lab Systems (Auckland, NZ). All agar plates, and liquid media containing antibiotics, were stored at $4{ }^{\circ} \mathrm{C}$; liquid media without antibiotics were stored at room temperature.

\section{H9}

Difco $^{\text {TM }}$ Middlebrook 7H9 broth was ordered from Becton Dickson. 7H9 broth was made up according to the manufacturer's instructions in $\mathrm{ddH}_{2} \mathrm{O}$, containing $0.05 \%$ Tween $^{\text {TM }} 80(\mathrm{v} / \mathrm{v})$, $0.05 \%$ glycerol (v/v) and $10 \%$ oleic acid, albumin, dextrose, catalase (OADC) supplement, and stored at $4{ }^{\circ} \mathrm{C}$

\subsubsection{Media supplements}

\section{Tween $^{\text {TM }} 80$}

Tween $^{\mathrm{TM}} 80$ was made up as a $20 \%(\mathrm{v} / \mathrm{v})$ stock solution using $\mathrm{ddH}_{2} \mathrm{O}$ and sterilised by autoclaving at $121^{\circ} \mathrm{C}$ for 30 minutes. Tween ${ }^{\mathrm{TM}} 80$ stocks were stored at room temperature. 


\section{D-arabinose}

D-arabinose was made up as a $100 \mathrm{mg} \cdot \mathrm{mL}^{-1}$ stock solution in $\mathrm{ddH}_{2} \mathrm{O}$ and filter sterilised through a $0.22 \mu \mathrm{M}$ nylon syringe filter unit. D-arabinose stocks were stored at $4^{\circ} \mathrm{C}$.

Oleic acid, albumin, dextrose, catalase enrichment (OADC)

OADC was purchased from Fort Richard Laboratories and was stored at $4{ }^{\circ} \mathrm{C}$

\section{Glycerol}

Glycerol was made up as a $10 \%(\mathrm{v} / \mathrm{v})$ stock and sterilised by autoclaving at $121{ }^{\circ} \mathrm{C}$ for 30 minutes. Glycerol stocks were stored at $4{ }^{\circ} \mathrm{C}$.

\section{Antibiotics}

All antibiotics used to select for or maintain plasmids/transposons were made up in $\mathrm{ddH}_{2} \mathrm{O}$ and filter sterilised through a $0.22 \mu \mathrm{M}$ nylon syringe filter before storage at $-20^{\circ} \mathrm{C}$, with the exception of hygromycin $B$, which was purchased as a $52.5 \mathrm{mg} \cdot \mathrm{mL}^{-1}$ aqueous solution and stored at $4{ }^{\circ} \mathrm{C}$. Antibiotic concentrations used for different bacterial strains are listed in Table 2.3.

Table 2.3: Antibiotic concentrations used for marker selection.

\begin{tabular}{cccc}
\hline \multirow{3}{*}{ Antibiotic } & \multirow{3}{*}{ Strain } \\
\cline { 2 - 4 } & & \multicolumn{2}{c}{ M. smegmatis } \\
\hline Ampicillin (Amp) & $200 \mu \mathrm{g} \cdot \mathrm{mL}^{-1}$ & $\mathrm{n} / \mathrm{a}$ & $\mathrm{n} / \mathrm{a}$ \\
Apramycin (Apr) & $50 \mu \mathrm{g} \cdot \mathrm{mL}^{-1}$ & $25 \mu \mathrm{g} \cdot \mathrm{mL}^{-1}$ & $25 \mu \mathrm{g} \cdot \mathrm{mL}^{-1}$ \\
Hygromycin B (Hyg) & $200 \mu \mathrm{g} \cdot \mathrm{mL}^{-1}$ & $20 \mu \mathrm{g} \cdot \mathrm{mL}^{-1}$ & $50 \mu \mathrm{g} \cdot \mathrm{mL}^{-1}$ \\
Kanamycin (Kan) & $50 \mu \mathrm{g} \cdot \mathrm{mL}^{-1}$ & $20 \mu \mathrm{g} \cdot \mathrm{mL}^{-1}$ & $50 \mu \mathrm{g} \cdot \mathrm{mL}^{-1}$ \\
\hline
\end{tabular}

\subsubsection{E. coli growth conditions}

Escherichia coli (E. coli) was routinely grown in LB or on LA (section 2.5) at $37^{\circ} \mathrm{C}$. Liquid cultures were aerated with shaking at $200 \mathrm{rpm}$ unless otherwise stated. All E. coli strains generated were stored as freezer stocks in $20 \%$ glycerol $(\mathrm{v} / \mathrm{v})$ at $-80^{\circ} \mathrm{C}$. Cultures of E. coli were prepared from freezer stocks by streaking out an inoculating loop of cells from the frozen stock onto appropriately supplemented LA plates. Plates were incubated overnight, with single colonies used to inoculate $5 \mathrm{~mL}$ of LB broth the next day. These $5 \mathrm{~mL}$ starter cultures were grown for a further 24 hours before being diluted 100-fold in fresh LB medium 
and grown for a further 24 hours. These cultures were used for generating chemically competent $E$. coli, or for plasmid preparation.

\subsubsection{Mycobacteria growth conditions}

\subsubsection{M. smegmatis $\mathrm{mc}^{2} 155$}

M. smegmatis $\mathrm{mc}^{2} 155$ was routinely grown in LB broth or on LA (section 2.5) at $37^{\circ} \mathrm{C}$. Liquid cultures were aerated with shaking at $200 \mathrm{rpm}$ unless otherwise stated. Tn611 transposon mutants of $M$. smegmatis $\mathrm{mc}^{2} 155$ were grown at $41{ }^{\circ} \mathrm{C}$ with shaking at $150 \mathrm{rpm}$. Liquid cultures of $M$. smegmatis $\mathrm{mc}^{2} 155$ were supplemented with $0.05 \%(\mathrm{v} / \mathrm{v})$ Tween $^{\mathrm{TM}} 80$ and $100 \mu \mathrm{g} \cdot \mathrm{mL}^{-1} \mathrm{D}$-arabinose (section 2.5.1) to minimise cell clumping (Allen, 1998; Anton et al., 1996). All M. smegmatis strains generated, except the transposon mutant collection, were stored as freezer stocks in $20 \%$ glycerol $(\mathrm{v} / \mathrm{v})$ at $-80^{\circ} \mathrm{C}$. The description of storage of the transposon mutant collection is described in section 2.7. Cultures of $M$. smegmatis $\mathrm{mc}^{2} 155$ were prepared from freezer stocks by streaking out an inoculating loop of cells from the frozen stock onto appropriately supplemented LA plates. Plates were incubated for $3-5$ days until colonies appeared. An individual colony was inoculated into $1 \mathrm{~mL}$ of appropriately supplemented LB broth and grown for 72 hours. The $1 \mathrm{~mL}$ starter cultures were then diluted 100 -fold in fresh LB broth and grown for a further 24 hours. These cultures of M. smegmatis $\mathrm{mc}^{2} 155$ were used for the generation of electrocompetant cells, or in assays.

\subsubsection{M. tuberculosis $\mathrm{H}_{37} \mathrm{Rv}$}

Growth and assaying of $M$. tuberculosis $\mathrm{H}_{37} \mathrm{Rv}$ was performed at University College Dublin, in Dublin Ireland, in the physical containment 3 (PC3) laboratory of Professor Stephen Gordon. M. tuberculosis $\mathrm{H}_{37} \mathrm{Rv}$ was routinely grown in $7 \mathrm{H} 9$ broth at $37{ }^{\circ} \mathrm{C}$, with liquid cultures aerated with shaking at $200 \mathrm{rpm}$ unless otherwise stated. M. tuberculosis $\mathrm{H}_{37} \mathrm{Rv}$ stocks were maintained by continuous culture in roller bottles as described by Bacon and Hatch (2009). 


\subsection{Molecular biology}

\subsubsection{DNA isolation and purification}

\subsubsection{CTAB genomic DNA isolation}

Genomic DNA (gDNA) was isolated from bacterial strains using cetyltrimethylammonium bromide (CTAB) as described by Bull et al. (2000). Briefly, $10 \mathrm{~mL}$ overnight liquid cultures of M. smegmatis $\mathrm{mc}^{2} 155$ (section 2.5.3.1) were spun down at $2700 \mathrm{xg}$ for 20 minutes to pellet cells, then re-suspended in $400 \mu \mathrm{L}$ of $1 \times$ TE buffer (Appendix section 8.1) and transferred to $1.5 \mathrm{~mL}$ micro-centrifuge tubes. Cells were heat killed for 20 minutes at $80^{\circ} \mathrm{C}$ in a heat block, then left in a rack to cool to room temperature. Cells were then incubated at $37^{\circ} \mathrm{C}$ for one hour with $0.5 \mathrm{mg}$ lysozyme. To the cells 0.15 volumes of $10 \%$ SDS (w/v) and 0.01 volumes of $10 \mathrm{mg} \cdot \mathrm{mL}^{-1}$ proteinase $\mathrm{K}$ (Appendix section 8.1 ) were added before being briefly vortexed then incubated at $65{ }^{\circ} \mathrm{C}$ for 10 minutes. Following this, 0.2 volumes of $5 \mathrm{M} \mathrm{NaCl}$ and 0.2 volumes of a prewarmed $\left(65^{\circ} \mathrm{C}\right) \mathrm{CTAB} / \mathrm{NaCl}$ (Appendix section 8.1) solution were added. The cells were vortexed until the solution turned white. The cells were then incubated at $65^{\circ} \mathrm{C}$ for 10 minutes. To purify the DNA, 1 volume chloroform was added to the cells followed by brief vortexing then spinning in a micro centrifuge for 8 minutes at $12,000 \mathrm{xg}$. The aqueous phase was removed and 0.6 volumes of isopropanol was added before a further spin for 15 minutes at $12,000 \mathrm{xg}$. The supernatant was removed and the DNA pellet washed with $1 \mathrm{~mL}$ cold $70 \%(\mathrm{v} / \mathrm{v})$ ethanol before a final spin for 5 minutes at $12,000 \mathrm{xg}$. The ethanol was removed and the pellet left to air dry before suspension of the DNA in $50 \mu \mathrm{L} d \mathrm{ddH}_{2} \mathrm{O}$ and storage at $-20^{\circ} \mathrm{C}$.

\subsubsection{Plasmid mini-preparation}

Small scale extraction of plasmid DNA from bacterial cells was performed using either the Zymo Research Zyppy ${ }^{\mathrm{TM}}$ Plasmid Mini-Prep kit or the Qiagen QIAprep Spin Miniprep kit according to the relevant manufacturer's instructions. Plasmid DNA was extracted from $3-5$ $\mathrm{mL}$ of an overnight culture of $E$. coli (section 2.5.2), eluted in $50 \mu \mathrm{L} \mathrm{dd}_{2} \mathrm{O}$ and stored at $-20^{\circ} \mathrm{C}$. 


\subsubsection{Plasmid midi-preparation}

Medium scale extraction of plasmid DNA from bacterial cells was performed using the Invitrogen ${ }^{\mathrm{TM}}$ PureLink ${ }^{\mathrm{TM}}$ HighPure Plasmid DNA Midi-Prep kit according to the manufacturer's instructions. Plasmid DNA was extracted from $50-100 \mathrm{~mL}$ of an overnight culture of $E$. coli

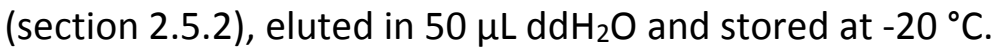

\subsubsection{Agarose gel electrophoresis}

DNA samples were run on $1 \%$ agarose $(w / v)$ gels in $1 x$ Tris acetate-EDTA (TAE) buffer (Appendix section 8.1). Samples were run with $1 x$ DNA loading buffer and alongside the HyperLadder $^{\mathrm{TM}} 1 \mathrm{~Kb}$ DNA ladder. Samples were electrophoresed using constant voltage at $120 \mathrm{~V}$ for $25-40$ minutes until suitable band separation was achieved.

\subsubsection{Agarose gel DNA extraction}

DNA bands were extracted from agarose gels following electrophoresis using the Zymoclean $^{\mathrm{TM}}$ Gel DNA Recovery Kit (Zymo Research) according to the manufacturer's instructions. DNA was eluted in $12 \mu \mathrm{LddH} \mathrm{H}_{2} \mathrm{O}$ and stored at $-20^{\circ} \mathrm{C}$.

\subsubsection{DNA purification}

DNA in solution was purified using the DNA Clean \& Concentrator ${ }^{\mathrm{TM}}-5$ kit (Zymo Research) according to the manufacturer's instructions. DNA was eluted in $12 \mu \mathrm{L} d \mathrm{dH}_{2} \mathrm{O}$ and stored at $-20^{\circ} \mathrm{C}$.

\subsubsection{DNA quantification}

DNA was quantified using a NanoDrop ${ }^{\circledR}$ ND-100 Spectrophotometer (NanoDrop Technologies, Auckland, NZ) and the NanoDrop ${ }^{\circledR} 3.1 .0$ software using the DNA setting. A $1 \mu \mathrm{L}$ aliquot of $\mathrm{ddH}_{2} \mathrm{O}$ was used to initialise the NanoDrop ${ }^{\circledR}$. A $1 \mu \mathrm{L}$ aliquot of $\mathrm{dd}_{2} \mathrm{O}$ was used to generate a blank reading before DNA concentrations were read at $230 \mathrm{~nm}$ using $1 \mu \mathrm{L}$ 
of sample. The ratio of the reading at $260 \mathrm{~nm}$ and $280 \mathrm{~nm}$ was recorded to assess sample purity.

\subsubsection{DNA sequencing}

DNA sequencing was performed by Macrogen Inc. (South Korea), using their standard sequencing service. A minimum of $200 \mathrm{ng}$ PCR or plasmid DNA was sent for sequencing in a minimum volume of $10 \mu \mathrm{L}$. Primers were sent at $10 \mu \mathrm{M}$ concentrations or synthesised by Macrogen Inc. using their primer synthesis service.

\subsubsection{Genetic manipulations}

\subsubsection{Primers}

Primers were designed using the Geneious 5.5.6 bioinformatics software and ordered from IDT. Primers were designed to include 15-25 nucleotides of the ends of the DNA sequence to be amplified, a restriction site if applicable, and a 5' CTAG motif. Primers were reconstituted in $\mathrm{ddH}_{2} \mathrm{O}$ to a concentration of $100 \mu \mathrm{M}$ and working stocks of $10 \mu \mathrm{M}$ were made up in $\mathrm{ddH}_{2} \mathrm{O}$ and stored at $-20^{\circ} \mathrm{C}$. Primers used in this study are listed in Table 2.4. 
Table 2.4: Primer sequences used in this study.

\begin{tabular}{|c|c|c|c|}
\hline Product & Primer Name & Sequence $\left(5^{\prime}-3^{\prime}\right)$ & $\begin{array}{l}T_{\mathrm{m}} \\
\left({ }^{\circ} \mathrm{C}\right)\end{array}$ \\
\hline \multirow[t]{2}{*}{ GFP } & GFP_RBS_F & $\begin{array}{c}\text { CTAGGGATCCTTTAAGAAGGAGATATACATATGAGT } \\
\text { AAAGGAGAA }\end{array}$ & 60.8 \\
\hline & GFP_R & CTAGGGATCCTTATTATTTGTATAGTTCATCCATGCC & 57.6 \\
\hline \multirow{2}{*}{$\begin{array}{l}\text { pRC20 MCS } \\
\text { primers }\end{array}$} & TetRo_int & GCCGACCTCTGCGGTTTGCT & 63.2 \\
\hline & GFP_int & AAGGACAGGGCCATCGCCAA & 62.0 \\
\hline \multirow{2}{*}{$\begin{array}{c}\text { nudC } \\
\text { (MSMEI_1904) }\end{array}$} & pRC20_1904_F & CTAGGGATCCATGAGCGAACACCGCACGTTCGG & 68.3 \\
\hline & pRC20_1904_R & CTAGGGATCCTCAGTCGAGTGCGGCCCAGGATT & 68.7 \\
\hline \multirow{3}{*}{ MSMEI_1905 } & pRC20_1905_F & $\begin{array}{l}\text { CTAGGEATCCATGACTACTGCTTCCAGCGAACTCAT } \\
\text { CATG }\end{array}$ & 65.7 \\
\hline & pRC20_1905_R & CTAGGGATCCTCAGCCGAGCTTGGCCTTGACCT & 68.9 \\
\hline & $\begin{array}{l}\text { 1905_BamHInull } \\
\text { _59mer }\end{array}$ & $\begin{array}{l}\text { TCAGCCGAGCTTGGCCTTGACCTGCTTGATGGTCGG } \\
\text { GTTGGTCAGCGTGGATCCGTCGG }\end{array}$ & 75.2 \\
\hline \multirow{2}{*}{ MSMEI_4267 } & pRC20_4267_F & CTAGGGATCCATGAAACTCCAGGTCACACA & 61.0 \\
\hline & pRC20_4267_R & CTAGGGATCCCTATAGATCGAGTGTTAATTTCGCG & 61.1 \\
\hline \multirow{2}{*}{ MSMEI_6092 } & pRC20_6092_F & CTAGGGATCCGTGACCACAGTGCAT & 62.2 \\
\hline & pRC20_6092_R & CTAGGGATCCCTAGATGGTGCTGCT & 61.5 \\
\hline \multirow{2}{*}{$\begin{array}{c}\text { inhA } \\
\text { (MSMEI_3070) }\end{array}$} & pRC20_3070_F & CTAGGGATCCATGACAGGCCTACTCGAAGG & 62.5 \\
\hline & pRC20_3070_R & CTAGGGATCCTCACAACAGCTGCGTGCTGG & 65.5 \\
\hline \multirow{2}{*}{$\begin{array}{c}\text { arr } \\
\left(M S M E I \_1186\right)\end{array}$} & pRC20_1186_F & CTAGGGATCCGTGGCGAATCCGCCG & 66.8 \\
\hline & pRC20_1186_R & CTAGGGATCCCTAGTCATAGATGACCGCCA & 63.0 \\
\hline \multirow{2}{*}{ MSMEI_3093 } & pRC20_3093_F & CTAGGGATCCGTGGACACCCGGTCGAT & 66.4 \\
\hline & pRC20_3093_R & CTAGGGATCCTCACAGTGCGTGCCGG & 66.2 \\
\hline \multirow{4}{*}{$\begin{array}{c}\text { katG } \\
\left(M S M E I \_6216\right)\end{array}$} & pRC20_6216_F & CTAGGGATCCTTGCCTGAGGATCGCC & 61.9 \\
\hline & pRC20_6216_R & CTAGGGATCCTCAGGCGACGTCGAA & 61.8 \\
\hline & 6216_int_F & CAACGGCAATCCGGACCCGCAGGCCTCGGC & 73.6 \\
\hline & 6216_int_R & TCCGGATTGCCGTTGGGGCCTTCGGGGTTG & 71.7 \\
\hline \multirow{2}{*}{$\begin{array}{l}\text { ligation- } \\
\text { mediated PCR }\end{array}$} & Salpt & $\underline{\text { TCGAGCTCGTGC }}$ & 47.0 \\
\hline & Salgd & TAGCTTATTCCTCAAGGCACGAGC & 58.8 \\
\hline \multirow{4}{*}{ IS6100 primers } & 3053 & GAACCGCTTCGCTGCCTTG & 45.0 \\
\hline & 3054 & AACCACCATTTCGCAGCAGC & 44.0 \\
\hline & OligoF & AAGAATTCATCGTTCCGTCCGTCCAATCTCC & 62.9 \\
\hline & OligoG & GAGCGACAGCCTACCTCTGACT & 60.4 \\
\hline
\end{tabular}

Sequences of primers used to amplify genes, or used for sequencing, along with their melting temperatures $\left(T_{\mathrm{m}}\right)$. Underlined bases represent restriction endonuclease recognition sites introduced into the primer. Bases in bold in primer GFP_RBS_F represent a shine-delgano ribosomal binding site sequence introduced into the primer. Italicised bases in sequences salgd and salpt represent homologous regions of these two oligonucleotides used to generate a linker during ligation-mediated PCR. 


\subsubsection{Polymerase chain reaction}

PCR was run using the conditions as listed in Table 2.5. All PCR mixes were prepared according to the manufacturer's instructions. AccuSure ${ }^{\mathrm{TM}}$ mix or Phusion ${ }^{\circledR}$ polymerase was used for reactions requiring high fidelity such as amplification of genes for downstream cloning into expression vectors. AccuSure ${ }^{\mathrm{TM}}$ was also used for all touchdown PCR and ligation-mediated PCR reactions. Biomix ${ }^{\mathrm{TM}}$ Red was used for inverse PCR and the overlap PCR of katG. Specific PCR reaction protocols used are listed in Table 2.6 - Table 2.9. All reactions used 100 - 200 ng of template; plasmid pll192_hsp60 was used as the template for GFP amplification; $M$. smegmatis $\mathrm{mc}^{2} 155$ gDNA (section 2.6.1.1) was used as the template for amplification of $M$. smegmatis genes; the left and right amplified fragments of katG were used as templates for the katG overlap PCR reaction; generation of the template for inverse PCR and ligation-mediated PCR are described in detail in sections 2.6.4 and 2.6.5 respectively. 
Table 2.5: PCR conditions for product amplification.

\begin{tabular}{|c|c|c|c|c|c|}
\hline Product & Primer pair & Protocol & Enzyme & $\begin{array}{l}\text { Annealing } \\
\text { Temp. }\left({ }^{\circ} \mathrm{C}\right)^{a}\end{array}$ & $\begin{array}{l}\text { Extension } \\
\text { time } \\
\text { (minutes) }\end{array}$ \\
\hline GFP & GFP_RBS_F/GFP_R & Touchdown & AccuSure ${ }^{\mathrm{TM}}$ & $68(-1) / 55$ & $1: 30$ \\
\hline $\begin{array}{c}\text { nudC } \\
\text { (MSMEI_1904) }\end{array}$ & $\begin{array}{l}\text { PRC20_1904_F/ } \\
\text { pRC20_1904_R }\end{array}$ & Touchdown & AccuSure $^{\mathrm{TM}}$ & $72(-1) / 62$ & $2: 10$ \\
\hline $\begin{array}{c}\text { MSMEI_1905 } \\
\text { (removing } \\
\text { BamHI site) }\end{array}$ & $\begin{array}{c}\text { pRC20_1905_F/ } \\
\text { 1905_BamHInull_59mer }\end{array}$ & Two-step & AccuSure ${ }^{\mathrm{TM}}$ & $\mathrm{n} / \mathrm{a}$ & $0: 40$ \\
\hline MSMEI_1905 & $\begin{array}{l}\text { pRC20_1905_F/ } \\
\text { pRC20_1905_R }\end{array}$ & Touchdown & AccuSure $^{\mathrm{TM}}$ & $71(-1) / 59$ & $0: 40$ \\
\hline MSMEI_4267 & $\begin{array}{l}\text { pRC20_4267_F/ } \\
\text { pRC20_4267_R }\end{array}$ & Touchdown & AccuSure $^{\mathrm{TM}}$ & $68(-1) / 57$ & $2: 10$ \\
\hline MSMEI_6092 & $\begin{array}{l}\text { pRC20_6092_F/ } \\
\text { pRC20_6092_R }\end{array}$ & Touchdown & AccuSure ${ }^{\mathrm{TM}}$ & $68(-1) / 58$ & $2: 00$ \\
\hline $\begin{array}{c}\text { inhA } \\
\text { (MSMEI_3070) }\end{array}$ & $\begin{array}{l}\text { pRC20_3070_F/ } \\
\text { pRC20_3070_R }\end{array}$ & Touchdown & AccuSure ${ }^{\mathrm{TM}}$ & $68(-1) / 57$ & $2: 10$ \\
\hline $\begin{array}{c}a r r \\
\text { (MSMEI_1186) }\end{array}$ & $\begin{array}{l}\text { pRC20_1186_F/ } \\
\text { pRC20_1186_R }\end{array}$ & Two-step & Phusion $^{\circledR}$ & $\mathrm{n} / \mathrm{a}$ & $0: 15$ \\
\hline MSMEI_3093 & $\begin{array}{l}\text { pRC20_3093_F/ } \\
\text { pRC20_3093_R }\end{array}$ & Two-step & Phusion $^{\circledR}$ & $\mathrm{n} / \mathrm{a}$ & $0: 25$ \\
\hline $\begin{array}{c}\text { katG left } \\
\text { fragment } \\
(M S M E I \text { 6216) }\end{array}$ & $\begin{array}{c}\text { pRC20_6216_F/ } \\
\text { 6216_int_R }\end{array}$ & Two-step & Phusion $^{\circledR}$ & $\mathrm{n} / \mathrm{a}$ & $0: 12$ \\
\hline $\begin{array}{c}\text { katG right } \\
\text { fragment } \\
(M S M E I \text { 6216) }\end{array}$ & $\begin{array}{c}\text { 6216_int_F/ } \\
\text { pRC20_6216_R }\end{array}$ & Two-step & Phusion $^{\circledR}$ & $\mathrm{n} / \mathrm{a}$ & $0: 23$ \\
\hline $\begin{array}{c}\text { katG } \\
\text { (MSMEI_6216) }\end{array}$ & $\begin{array}{l}\text { pRC20_6216_F/ } \\
\text { pRC20_6216_R }\end{array}$ & Overlap & Biomix $^{\mathrm{TM}}$ Red & \multicolumn{2}{|c|}{ See Table 2.9} \\
\hline Inverse PCR & $3053 / 3054$ & Three-step & Biomix $^{T M}$ Red & 44 & $2: 00$ \\
\hline $\begin{array}{c}\text { Ligation- } \\
\text { mediated PCR }\end{array}$ & salgd/OligoF or OligoG & Three-step & AccuSure $^{T M}$ & 55 & $2: 20$ \\
\hline
\end{tabular}

a Times for touchdown PCR reactions are in the format: phase I annealing temperature (temperature change per step) / phase II annealing temperature. 


\section{Two-step PCR}

A two-step PCR protocol was used when the $T_{\mathrm{m}}$ of one or both primers in a primer pair was higher than $70{ }^{\circ} \mathrm{C}$ when using Phusion ${ }^{\circledR}$ polymerase, or $72{ }^{\circ} \mathrm{C}$ when using any other DNA polymerase.

Table 2.6: Two-step PCR thermal cycle.

\begin{tabular}{cccc}
\hline Step & ${\text { Temperature }\left({ }^{\circ} \mathrm{C}\right)^{\mathrm{a}}}$ & ${\text { Time }(\min )^{\mathrm{a}}}$ & No. of cycles \\
\hline Initial Denature & $98 / 95$ & $2: 00 / 7: 00$ & 1 \\
\hline Denaturation & $98 / 95$ & $0: 30$ & 30 \\
Extension & $68 / 72$ & $0: 30 / 1: 30 \mathrm{~Kb}^{-1}$ & \\
\hline Final Extension & $68 / 72$ & $10: 00$ & 1 \\
\hline
\end{tabular}

${ }^{\text {a }}$ Denaturation temperature and time, and extension temperature and time were dependant on the polymerase used (Phusion ${ }^{\circledR} /$ AccuSure $^{\mathrm{TM}}$ ).

\section{Three-step PCR}

A three-step PCR was used when the average $T_{\mathrm{m}}$ of a primer pair was below $65^{\circ} \mathrm{C}$ and the $T_{\mathrm{m}}$ of the primers were within $2^{\circ} \mathrm{C}$ of each other.

Table 2.7: Three-step PCR thermal cycle.

\begin{tabular}{cccc}
\hline Step & Temperature $\left.^{\circ}{ }^{\circ} \mathbf{C}\right)^{\text {a }}$ & Time $(\min )^{\text {a }}$ & No. of cycles \\
\hline Initial Denature & 95 & $2: 00 / 7: 00$ & 1 \\
Denaturation & 95 & $0: 30$ & \\
Annealing & See Table 2.5 & $0: 30$ & 30 \\
Extension & 72 & $0: 30 / 1: 30 \mathrm{~Kb}^{-1}$ & \\
\hline Final Extension & 72 & $10: 00$ & 1
\end{tabular}

${ }^{\text {a }}$ Denaturation and extension time were dependant on the polymerase mix used (Biomix ${ }^{\mathrm{TM}}$ Red / AccuSure $\left.{ }^{\mathrm{TM}}\right)$.

\section{Touchdown PCR}

A Touchdown PCR was used to amplify targets when the average $T_{\mathrm{m}}$ of a primer pair was greater than $65^{\circ} \mathrm{C}$ and less than $72{ }^{\circ} \mathrm{C}$ and/or when the temperature difference within a primer pair was more than $2{ }^{\circ} \mathrm{C}$. All touchdown PCR reactions were performed with AccuSure ${ }^{\mathrm{TM}}$. 
Table 2.8: Touchdown PCR thermal cycle.

\begin{tabular}{ccccc}
\hline Phase & Step & Temperature $\left({ }^{\circ} \mathbf{C}\right)^{\text {a }}$ & ${\text { Time }(\mathbf{m i n})^{\text {a }}}$ & No. of cycles \\
\hline & Initial Denature & 95 & $7: 00$ & 1 \\
& $\begin{array}{c}\text { Denaturation } \\
\text { Phase I }\end{array}$ & 95 & $0: 30$ & \\
& $\begin{array}{c}\text { Touchdown } \\
\text { Annealing } \\
\left(-1{ }^{\circ} \mathrm{C} / \text { cycle }\right)\end{array}$ & See Table 2.5 & $0: 30$ & $8-15$ cycles \\
& Extension & 72 & & \\
& Denaturation & 95 & $1: 30 \mathrm{~Kb}^{-1}$ & \\
Phase II & Annealing & See Table 2.5 & $0: 30$ & \\
& Extension & 72 & $0: 30$ & 25 cycles \\
& Final Extension & 72 & $1: 30 \mathrm{~Kb}^{-1}$ & \\
\hline
\end{tabular}

${ }^{\text {a }}$ The number of cycles performed during phase I was equal to the number required to get from the phase I annealing temperature to the phase II annealing temperature using $1{ }^{\circ} \mathrm{C}$ increments per cycle.

\section{Overlap PCR}

Overlap PCR was used to remove the BamHI restriction site from the katG gene MSMEI_6216. Phase I used 1x Biomix ${ }^{\mathrm{TM}}$ Red, equimolar katG left and right fragments as the template (70 nM each), and $\mathrm{ddH}_{2} \mathrm{O}$ to bring the reaction volume to $25 \mu \mathrm{L}$. The kat $G$ left and right fragments were amplified using the protocols described in Table 2.5, and cleaned and concentrated as described in section 2.6.1.6, before use in the overlap PCR reaction. The primers listed in Table 2.5 were added prior to starting phase II, in a volume equal to that of the reaction in phase $\mathrm{I}$, containing $1 \times \mathrm{Biomix}^{\mathrm{TM}}$ Red and sufficient $d \mathrm{dH}_{2} \mathrm{O}$ to bring the final reaction volume to $50 \mu \mathrm{L}$.

Table 2.9: Overlap PCR thermal cycle.

\begin{tabular}{ccccc}
\hline Phase & Step & Temperature $\left({ }^{\circ} \mathbf{C}\right)$ & Time $(\mathbf{m i n})$ & No. of cycles \\
\hline & Initial Denature & 95 & $2: 00$ & 1 \\
\hline \multirow{3}{*}{ Phase I } & Denaturation & 95 & $0: 30$ & 15 cycles \\
& Annealing & 39 & $0: 30$ & \\
& Extension & 72 & $1: 00$ & \\
\multirow{4}{*}{ Phase II } & $95^{\circ} \mathrm{C}$ hold to add reagents for phase II & 25 cycles \\
& Denaturation & 95 & $0: 30$ & \\
& Annealing & $0: 30$ & $0: 30$ & 1 \\
\hline
\end{tabular}




\subsubsection{Restriction endonuclease digestions}

Restriction endonuclease reactions were run according to the manufacturer's instructions with the supplied buffers unless otherwise specified. Restriction endonuclease reactions were heat inactivated according to the manufactures instructions where specified, and cleaned and concentrated as described in section 2.6.1.6. Completed restriction reactions were electrophoresed (section 2.6.1.4) alongside undigested control DNA to confirm successful digestion.

\subsubsection{Blunting of DNA ends}

T4 DNA polymerase was used to blunt the ends of DNA fragments according to the manufacturer's protocol. NEBuffer 2 was used in place of the supplied CutSmart ${ }^{\mathrm{TM}}$ buffer. Blunting reactions were inactivated with $10 \mathrm{mM}$ ethylenediaminetetraacetic acid (EDTA) at $75^{\circ} \mathrm{C}$ for 20 minutes.

\subsubsection{DNA phosphatase treatment}

Antarctic phosphatase was used to remove the phosphates from the $5^{\prime}$ ends of DNA fragments according to the manufacturer's protocol.

\subsubsection{DNA ligations}

T4 DNA ligase was used to ligate DNA fragments together according to the manufacturer's protocol. Ligations were run overnight at room temperature and diluted five-fold prior to use in transforming chemically competent cells.

\subsubsection{Generation of substrate for inverse PCR}

Inverse PCR was used to identify transposon insertion sites in the genome of $M$. smegmatis $\mathrm{mc}^{2} 155$ and was performed as described by Billman-Jacobe et al. (2006). Briefly, gDNA was isolated from mutant strains as described in section 2.6.1.1. Digests were performed with $600 \mathrm{ng}$ gDNA and the restriction enzymes Rsal or Eagl in $20 \mu \mathrm{L}$ reactions according to the 
manufacturer's protocol (section 2.6.3.3). The reactions were heat inactivated but not cleaned and concentrated. An $18 \mu \mathrm{L}$ aliquot of the restriction digest was then transferred to a ligation reaction setup according to the manufacturer's instructions (section 2.6.3.6) in a total volume of $50 \mu \mathrm{L}$. Ligations were incubated overnight at room temperature, and then stored at $-20^{\circ} \mathrm{C}$. For inverse PCR reactions $8 \mu \mathrm{L}$ ligation mixture was used in a total reaction volume of $25 \mu \mathrm{L}$.

\subsubsection{Generation of substrate for ligation mediated PCR}

Ligation-mediated PCR was used to identify insertion sites in the genome of $M$. smegmatis $\mathrm{mc}^{2} 155$ and was performed essentially as described by Prod'hom et al. (1998). Briefly, the linker was made by annealing the oligonucleotides salgd and salpt (Table 2.4) in equimolar $(25 \mu \mathrm{M})$ concentrations in $1 \mathrm{x}$ Phusion ${ }^{\circledR}$ high-fidelity PCR buffer in a $200 \mu \mathrm{L}$ PCR tube. The mixture was heated to $80^{\circ} \mathrm{C}$ in a thermal cycler and cooled to $4{ }^{\circ} \mathrm{C}$ over one hour. Digests were performed with $250 \mathrm{ng}$ gDNA isolated from mutants as describe in section 2.6.1.1 using the restriction enzyme Sall in $20 \mu \mathrm{L}$ reactions according to the manufacturer's instructions (section 2.6.3.3). Digest reactions were heat inactivated but not cleaned and concentrated. The linker was ligated to the Sall digested gDNA (section 2.6.3.6) according to the manufacturer's instructions for one hour at $16{ }^{\circ} \mathrm{C}$. Ligation reactions were performed in 10 $\mu \mathrm{L}$ volumes containing $5 \mu \mathrm{L}$ of the digest reaction mixture and 25 pmol linker. Ligations were heat inactivated at $65^{\circ} \mathrm{C}$ for 10 minutes then re-digested with Sall in a $15 \mu \mathrm{L}$ reaction according to the manufacturer's instructions. Digestion reactions were heat inactivated but not cleaned and concentrated, and diluted 10-fold in $\mathrm{dd}_{2} \mathrm{O}$. A $5 \mu \mathrm{L}$ aliquot of the diluted restriction digest mixture was used as template in the ligation-mediated PCR (section 2.6.3.2) in a total reaction volume of $50 \mu \mathrm{L}$.

\subsubsection{Transformation of E. coli DH5 $\alpha$}

\subsubsection{Generation of chemically competent $E$. coli cells}

Generation of chemically competent E. coli $\mathrm{DH} 5 \alpha$ cells was performed as described by Sambrook \& Russell (2001) with the following alterations. Cells were re-suspended in twice 
the volume of $0.1 \mathrm{M} \mathrm{CaCl}_{2}, 14 \%(\mathrm{v} / \mathrm{v})$ glycerol than stated, and frozen in $100 \mu \mathrm{L}$ aliquots at $-80^{\circ} \mathrm{C}$.

\subsubsection{Heat shock transformation of $E$. coli cells}

Chemically competent $E$. coli $\mathrm{DH} 5 \alpha$ were transformed by heat shocked as described by Sambrook \& Russell (2001). Briefly, aliquots of cells stored at $-80^{\circ} \mathrm{C}$ were thawed on ice for 20 minutes following the addition of up to $10 \mu \mathrm{L}$ of the DNA to be transformed. A sample of E. coli was also transformed with $10 \mu \mathrm{L} \mathrm{dd}_{2} \mathrm{O}$ to act as a negative control. Cells were heat-shocked in a heat block at $42^{\circ} \mathrm{C}$ for 90 seconds, and then chilled on ice for five minutes. Following heat-shock $900 \mu \mathrm{L}$ LB was added to the cells before recovery for 1 hour at $37^{\circ} \mathrm{C}$ before plating on LA supplemented with the appropriate antibiotics (section 2.5.1).

\subsubsection{Transformation of $M$. smegmatis $\mathbf{m c}^{2} 155$}

\subsubsection{Generation of electrocompetant $M$. smegmatis cells}

Generation of electroporation competent M. smegmatis $\mathrm{mc}^{2} 155$ cells was performed as described by Goude and Parish (2009). Cells were frozen in $200 \mu \mathrm{L}$ aliquots at $-80^{\circ} \mathrm{C}$.

\subsubsection{Electroporation of $M$. smegmatis cells}

Electrocompetant cells of $M$. smegmatis $\mathrm{mc}^{2} 155$ were transformed by electroporation as described by Goude and Parish (2009). Briefly, $200 \mu \mathrm{L} 10 \%$ glycerol (v/v) cooled to $-20{ }^{\circ} \mathrm{C}$ was added to $200 \mu \mathrm{L}$ of frozen cells which were thawed on ice for 1.5 hours in $2 \mathrm{~mm}$ gap electroporation cuvettes. A maximum of $5 \mu \mathrm{L}$ of the plasmid to be transformed was added before electroporation, which was performed at $2500 \mathrm{~V}, 25 \mu \mathrm{F}$, and $1000 \Omega$. Cells were then added to $600 \mu \mathrm{L}$ LB in $1.5 \mathrm{~mL}$ micro-centrifuge tubes and recovered for two hours at $37^{\circ} \mathrm{C}$ before plating on LA supplemented with the appropriate antibiotics (section 2.5.1). 


\subsubsection{Construction of strain MRC10}

The plasmid pRC10 was constructed by removing the nitrile inducible operon from plasmid pNIT-1 as depicted in Figure 2.2. A digest was setup with $1 \mu \mathrm{g}$ of plasmid pNIT-1 and the restriction enzymes $\mathrm{Xbal}$ and Notl according to the manufacturer's instructions (section 2.6.3.3). Both single $\mathrm{Xbal}$ and Notl digestion reactions, as well as a double $\mathrm{Xbal} /$ Notl reaction were performed. The $4.8 \mathrm{~Kb}$ band in the $\mathrm{Xbal} / \mathrm{Notl}$ double digestion reaction, which was the backbone of the pNIT-1 plasmid, was gel extracted (section 2.6.1.5). To blunt the ends of the DNA fragment, 250 ng of the pNIT-1 plasmid backbone was treated with T4 DNA polymerase (section 2.6.3.4). The blunted pNIT-1 plasmid backbone was then ligated with T4 DNA ligase (section 2.6.3.6) under dilute conditions $\left(0.5 \mathrm{ng} \cdot \mathrm{mL}^{-1} \mathrm{DNA}\right)$ to promote circularisation. Ligation products were transformed into E. coli $\mathrm{DH} 5 \alpha$ (section 2.6.6.2), and selected on LA with $50 \mu \mathrm{g} \cdot \mathrm{mL}^{-1}$ kanamycin. Several individual transformants were grown up overnight and plasmid mini-prepped (section 2.6.1.2). These plasmids were digested with Spel (section 2.6.3.3), which cuts the pNIT-1 backbone once, to check the size of the plasmid. This plasmid was called pRC10, and was transformed into M. smegmatis $\mathrm{mc}^{2} 155$ (section 2.6.7.2) to generate strain MRC10.
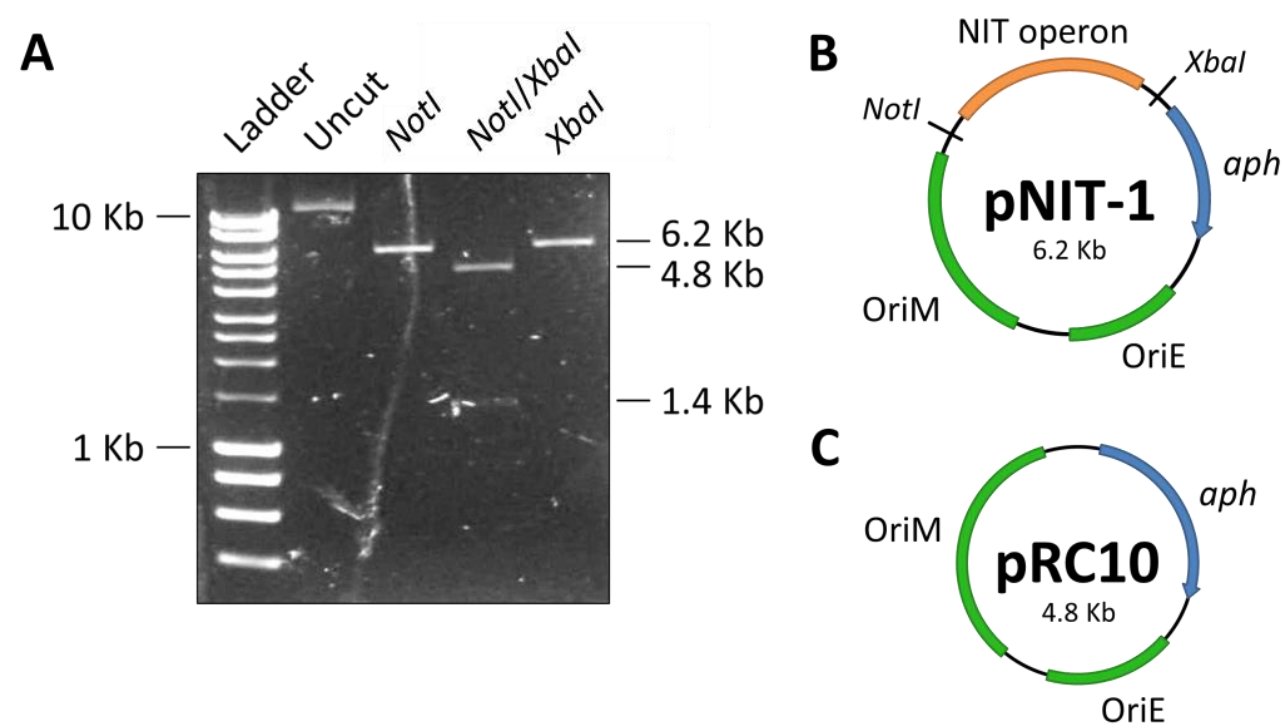

Figure 2.1: Digestion of pNIT-1 and plasmid maps. (A) Single and double restriction endonuclease digestions of plasmid pNIT-1. Single digests with Notl and $\mathrm{Xbal}$ resulted in a $6.2 \mathrm{~Kb}$ fragment. The double digest resulted in a 4.8 and $1.4 \mathrm{~Kb}$ fragment (pNIT-1 plasmid backbone and nitrile inducible operon respectively). Plasmid maps of (B) pNIT-1 and (C) pRC10 with relevant features shown: OriE, E. coli pUC origin of replication; OriM, mycobacterial pAL5000 origin of replication; aph, kanamycin resistance gene; NIT operon, nitrile inducible operon. 


\subsubsection{Construction of plasmid pRC20}

Plasmid pRC20 was constructed to enable tetracycline inducible gene expression in M. smegmatis $\mathrm{mc}^{2} 155$, with semi-quantitative measurement of gene expression provided by a green fluorescent protein (GFP) reporter gene. The plasmid pRC20 was constructed by removing the luciferase genes ( $u x A B)$ from the plasmid pKW08_Lx, and replacing it with the GFP gene as depicted in Figure 2.2. A digest was performed with $3 \mu \mathrm{g}$ of plasmid pKW08_Lx and the restriction enzymes BamHI and HindIII according to the manufacturer's instructions (section 2.6.3.3). Both single BamHI and HindIII digestions, as well as a double BamHI/HindIII digestion were performed. The $5.9 \mathrm{~Kb}$ band that corresponded to the pKW08_Lx plasmid backbone was gel extracted (section 2.6.1.5). The GFP gene was amplified by PCR from plasmid pLL192_hsp60 as described in section 2.6.3.2, and digested with BamHI as described in section 2.6.3.3. The GFP PCR product was cleaned and concentrated as described in section 2.6.1.6. GFP was ligated into the backbone of plasmid pKW08_Lx as described in section 2.6.3.6 using a 3:1 insert to vector molar ratio. Ligations were transformed into $E$. coli $\mathrm{DH} 5 \alpha$ (section 2.6.6.2), and selected on LA with $50 \mu \mathrm{g} \cdot \mathrm{mL}^{-1}$ hygromycin B. Several individual transformants were grown up overnight and plasmid midi-prepped (section 2.6.1.3). These plasmids were digested with BamHI (section 2.6.3.3), which cuts the pKW08_Lx backbone once, to check the size of the plasmid. This plasmid was called pRC20 and was further verified by sequencing with the primer TetRO_int (Table 2.4) which targets the multiple cloning site, and reads into the downstream GFP gene. 
A

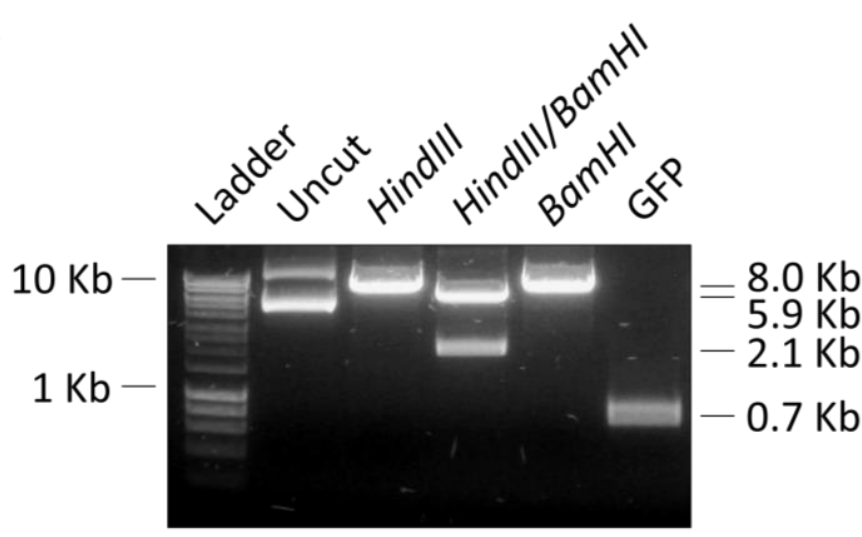

B

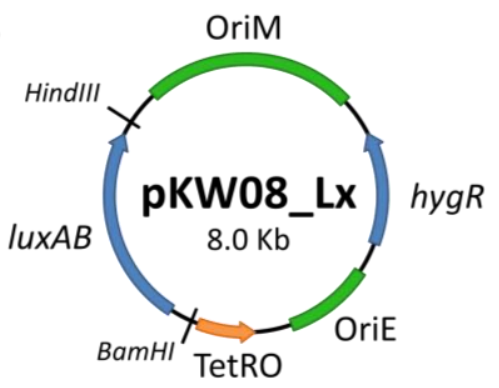

C

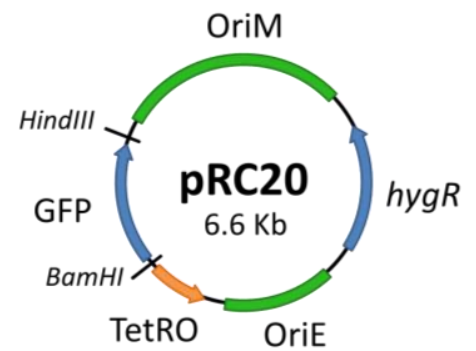

Figure 2.2: Generation of pKW08 derivative pRC20. (A) Single and double restriction endonuclease digestions of plasmid pKW08_Lx were performed. Single digests with HindIII and BamHI resulted in an $8.0 \mathrm{~Kb}$ fragment. A double digest resulted in 5.9 and $2.1 \mathrm{~Kb}$ fragments (pKW08_Lx plasmid backbone and luxAB genes respectively). The GFP gene was also digested with BamHI/HindIII. Plasmid map of (B) pKW08_Lx and (C) pRC20 with relevant features shown: OriE, E. coli pUC origin of replication; OriM, mycobacterial PAL5000 origin of replication; hygR, hygromycin B resistance gene; lux $A B$, luciferase genes $A$ and $B ; G F P$, green fluorescent protein; TetRO, tetracycline regulon including the gene $t e t R$, the operator tet $O$, and the promoter $\mathrm{P}_{\text {tet. }}$

\subsubsection{Construction of $\mathrm{pRC} 20$ gene insert vectors}

Genes were cloned into pRC20 to generate the expression vectors for the complementation and overexpression assays. Gene inserts were amplified by PCR as described in section 2.6.3.2, and were digested along with plasmid pRC2O with $\mathrm{BamHI}$ as described in section 2.6.3.3. To decrease self-ligation of the plasmid backbone, pRC20 was treated with Antarctic phosphatase as described in section 2.6.3.5. Gene inserts were then ligated into the pRC20 backbone using T4 DNA ligase as described in section 2.6.3.6, using a 3:1 insert to vector molar ratio. Ligations were transformed into $E$. coli $\mathrm{DH} 5 \alpha$ (section 2.6.6.2), and selected on LA with $50 \mu \mathrm{g} \cdot \mathrm{mL}^{-1}$ hygromycin B. Several transformants were selected and grown up in overnight cultures as described in section 2.5.2, with the cultures used for plasmid mini-preparation the next day (section 2.6.1.2). These isolated plasmids were sent for 
sequencing using the primer TetRO_int to confirm gene insert orientation, and sequence fidelity. Correct plasmid constructs were transformed into the relevant strains as described in section 2.6.7.2.

\subsubsection{0. $\quad$ Random transposon mutagenesis of $M$. smegmatis mc $^{2} 155$}

Random transposon mutants of $M$. smegmatis $m^{2} 155$ were generated using the pCG79 plasmid as described by Guilhot et al. (1994). Briefly, electrocompetent M. smegmatis $\mathrm{mc}^{2} 155$ cells were transformed with plasmid pCG79 which harbours the Tn611 transposon (Figure 3.1) and selected on LA supplemented with $20 \mu \mathrm{g} \cdot \mathrm{mL}^{-1}$ kanamycin at $30{ }^{\circ} \mathrm{C}$ for $4-5$ days. Several successful transformants were colony purified by re-streaking on fresh LA and left for another $4-5$ days at $30^{\circ} \mathrm{C}$. Cells were scraped off the plates and re-suspended in supplemented LB (section 2.5) containing $14 \%$ glycerol (v/v). Dilutions of $1 \times 10^{-2}-1 \times 10^{-6}$ of the cell suspensions were frozen down at $-80{ }^{\circ} \mathrm{C}$. Aliquots of the dilutions were plated in duplicate on fresh LA plates, and incubated at $41{ }^{\circ} \mathrm{C}$ to determine the optimal dilution for obtaining individual transposon mutants (approximately 100-200 colonies per plate). Further mutants were then generated by plating the optimal dilution on supplemented LA plates with incubation at $41^{\circ} \mathrm{C}$ for $3-5$ days.

\subsection{Transposon mutant collection construction}

The transposon mutant collection was generated as a mother stock that was sub inoculated to generate the daughter stock. The daughter stock was used for screening purposes. The mother stock was reserved as a backup for regenerating plates from the daughter stock if a plate was damaged, lost, or contaminated, etc.

\subsubsection{Generation of mother stock}

Each plate of the transposon mutant collection contained both random transposon mutants and a control strain MRC10 (section 2.6.8) as depicted in Figure 2.3. The control strain MRC10 was built into the library to provide a standard against which the other mutants on 
the plate could be compared, enabling comparison of different mutants across separate plates of the collection, and between different conditions. MRC10 was used as the control as it met the minimum standards for growing under assay conditions (kanamycin resistant). Random transposon mutants of $M$. smegmatis $\mathrm{mc}^{2} 155$ were generated as described in section 2.6.9.1, and transferred to individual wells of 384-well plates containing $50 \mu \mathrm{L}$ of supplemented LB (section 2.5) using sterile toothpicks. A $50 \mu \mathrm{L}$ aliquot of a mid-log phase culture of the control strain MRC10 was added to each of the control wells, and $50 \mu \mathrm{LddH} \mathrm{H}_{2} \mathrm{O}$ was added to the outside wells. Plates were wrapped in plastic cling film to prevent evaporation, and incubated at $41{ }^{\circ} \mathrm{C}$ with shaking at $150 \mathrm{rpm}$ for 48 hours prior to the addition of $35 \mu \mathrm{L}$ of $80 \%(\mathrm{v} / \mathrm{v})$ glycerol to each well. Plates were sealed with AlumaSeal ${ }^{\circledR} \mathrm{CS}$ aluminium plate seals (Sigma-Aldrich) before brief vortexing, and storage at $-80{ }^{\circ} \mathrm{C}$. This process was repeated for 32 separate 384 -well plates to generate the mother stock of the complete transposon mutant collection of 7680 mutants. The plates of the mother stock were numbered M01 - M32.

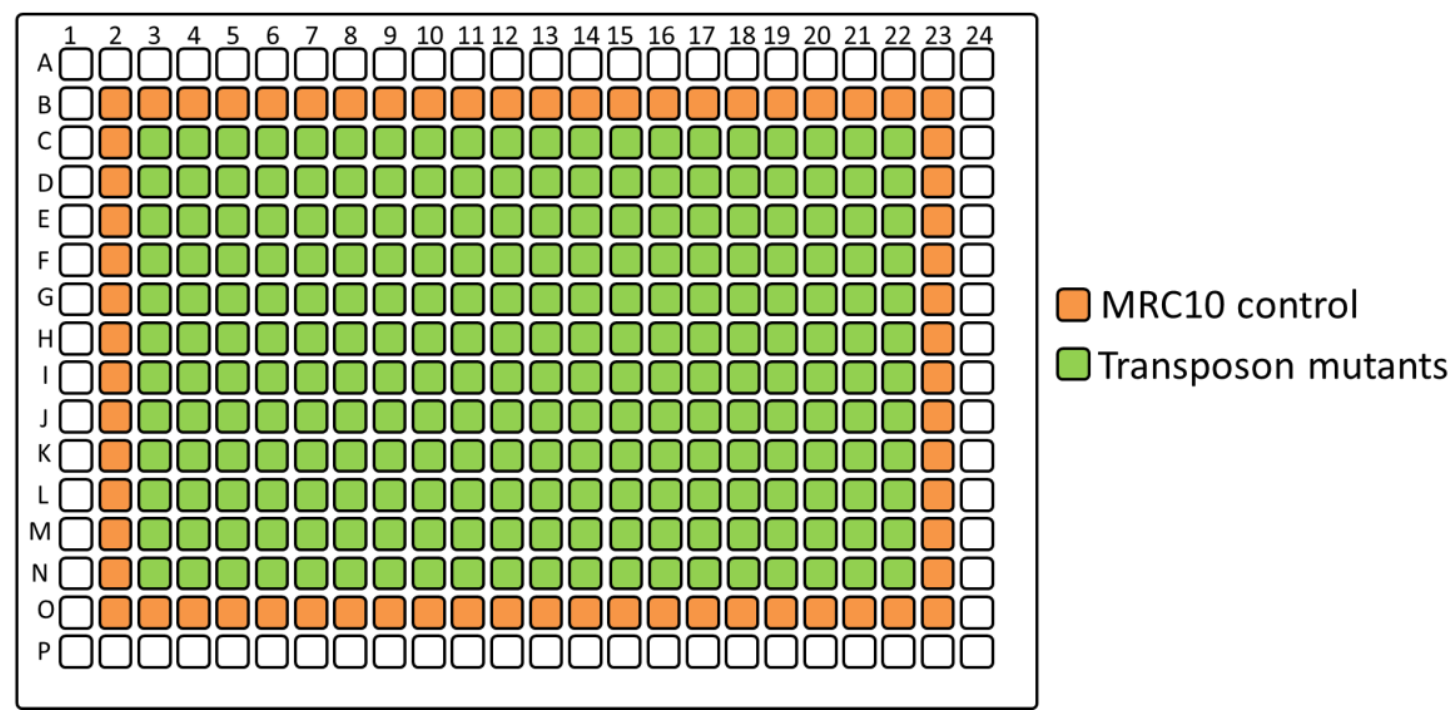

Figure 2.3: Layout of transposon mutant collection plates. Each plate of the collection contained 240 individual transposon mutants with each well inoculated with a separate mutant strain. The control strain MRC10 was added to the second outermost ring of wells. 


\subsubsection{Generation of daughter stock}

The daughter stock was generated before the addition of glycerol and freezing down of the mother plates for the first time. Plates of the daughter stock were generated by sub inoculating from the mother stock plates to fresh 384-well plates setup as descried in section 2.7.1. Sub inoculation was performed by pinning with 384-pin long pin RePads ${ }^{\circledR}$ (Singer). RePads $^{\circledR}$ were processed after each use as described in appendix section 1 . Plates were wrapped in plastic cling film and incubated at $41{ }^{\circ} \mathrm{C}$ with shaking at $150 \mathrm{rpm}$ for 48 hours before the addition of glycerol and freezing as in section 2.7.1. This process was repeated to generate a daughter plate for each mother plate of the transposon mutant collection. Plates of the daughter stock were labelled D01 - D32 with the numbers corresponding to the plates in the mother stock.

\subsubsection{Generation of mini-hit libraries}

The transposon mutant collection was assayed for drug hypersensitivity, as described in section 2.8.1, with the most sensitive mutants from the primary assay transferred to smaller mini-hit libraries. The 120 most sensitive mutants for each drug identified in the primary assay for drug hypersensitivity were picked into mini-hit libraries in the layout described in Figure 2.4. Three mini-hit libraries were constructed, one for isoniazid, one for rifampicin, and one for bedaquiline. The mini-hit libraries consisted of two 96-well plates, each with 60 transposon mutants corresponding to the 60 most sensitive mutants identified from the transposon mutant libraries $A$ and $B$ respectively. The mini-hit libraries plates contained $100 \mu \mathrm{L}$ supplemented LB (section 2.5) in the inner 60 wells, and $100 \mu \mathrm{L} \mathrm{ddH}_{2} \mathrm{O}$ in the outermost wells. The 120 most sensitive mutants were picked into individual wells of the mini-hit libraries using sterile toothpicks. Mutants were transferred into the mini-hit library in the order that they appeared in the complete transposon mutant collection. Following strain sub-inoculation from the transposon mutant collection, plates were grown at $41^{\circ} \mathrm{C}$ with shaking at $150 \mathrm{rpm}$ for 48 hours. To each well of the mini-hit libraries $70 \mu \mathrm{L} 80 \%$ glycerol ( $\mathrm{v} / \mathrm{v})$ was added, the plates were sealed with AlumaSeal ${ }^{\circledR}$ CS plate seals and briefly vortexed before storage at $-80^{\circ} \mathrm{C}$. 


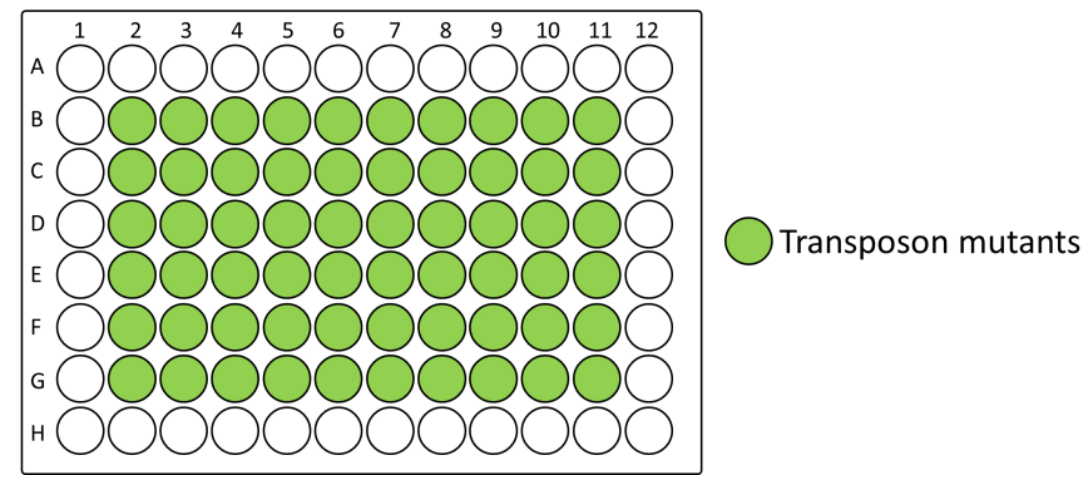

Figure 2.4: Layout of mini-hit library plates. Each plate of the mini-hit library contained 60 transposon mutants in the internal wells.

\subsection{Growth-inhibition assays}

\subsubsection{Assay for Drug hypersensitivity}

The transposon mutant collection was assayed for drug hypersensitivity using a primary high-throughput assay that utilised resazurin reduction as a surrogate measure of growth-inhibition. A secondary assay was run on the most sensitive mutants identified in the primary assay to validate their sensitivity. The secondary assay used endpoint optical density at $600 \mathrm{~nm}\left(\mathrm{OD}_{600}\right)$ to determine growth-inhibition. All assays using transposon mutants were assayed at $41{ }^{\circ} \mathrm{C}$ in order to maintain the transposon insertion while under the potential selective pressure caused by drug treatment.

\subsubsection{Primary assay for drug hypersensitivity}

For the primary assay for drug hypersensitivity the transposon mutant collection was split into two halves (Library A and Library B) with 16 plates per library. These libraries were assayed separately and their results processed independently.

The plates of a mutant library were sub-inoculated from the frozen daughter stock by pinning into fresh 384-plates setup as in section 2.7.1. Plates were wrapped in cling film and incubated at $41{ }^{\circ} \mathrm{C}$ with shaking at $150 \mathrm{rpm}$ for 72 hours. Plates were then sub-inoculated 
into fresh 384-well plates setup identically, and incubated for a further 24 hours. Plates were then used to sub-inoculate 384-well plates setup to assay for drug sensitivity as described in Figure 2.5, using the screening concentration of drug which was determined as described in section 3.2.3.1.

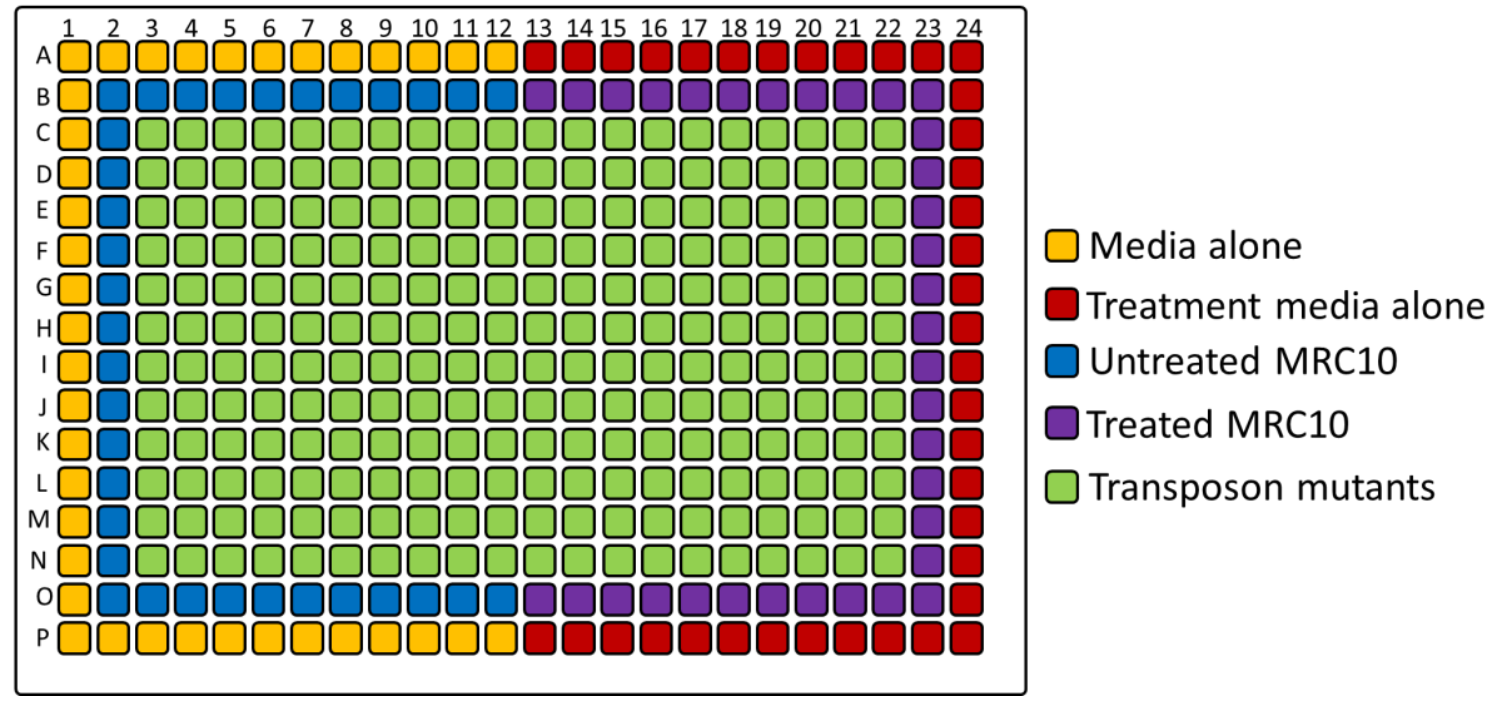

Figure 2.5: Plate setup for primary assays for drug hypersensitivity. The outermost wells were split into two halves, the media alone and treatment media controls. The MRC10 controls were also split into two, the untreated MRC10 controls and the treated MRC10 controls. The media alone and untreated MRC10 controls received no drug treatment in all assays, while the treatment media and treated MRC10 controls received the same treatment as the transposon mutants.

Assay plates were wrapped in cling film then incubated for 48 hours at $41{ }^{\circ} \mathrm{C}$ with shaking at $150 \mathrm{rpm}$. To each well 0.4 volumes of $0.03 \%$ resazurin sodium salt (w/v) (appendix section 8.1) was then added. Plates were left for a further two hours at $41{ }^{\circ} \mathrm{C}$ without shaking, before reading the fluorescence of the wells of each plate using a PerkinElmer EnSpire ${ }^{\circledR}$, 2300 multilable reader. Fluorescence measurements were performed at an excitation wavelength of $530 \mathrm{~nm}$ and an emission wavelength of $590 \mathrm{~nm}$. The primary assay for drug hypersensitivity was run three independent times for each drug and for media alone. Processing of the resulting data is described in section 3.2.3. 


\subsubsection{Secondary assay for drug-hypersensitivity}

The mini-hit libraries constructed as described in section 2.7.3 were assayed for drug hypersensitivity using the secondary assay. The same drug concentration was used in the secondary assay as was used in the primary assay (section 3.2.3.1).

The frozen mini-hit library stocks were used to sub-inoculate new 96-well plates setup as described in section 2.7.3. Sub-inoculation of the mini-hit libraries was performed using a metal 96-pin metal pinning tool, which was processed after each pinning as described in appendix section 1. Plates were wrapped in cling film and incubated at $41{ }^{\circ} \mathrm{C}$ for 48 hours, before pinning into fresh 96-well plates setup as described in section 2.7 .3 , and incubated for a further 24 hours. Assay plates were setup containing either $100 \mu \mathrm{L}$ media, or $100 \mu \mathrm{L}$ treatment containing the drug at the screening concentration, in every well of a 96-well plate as described in Figure 2.6.

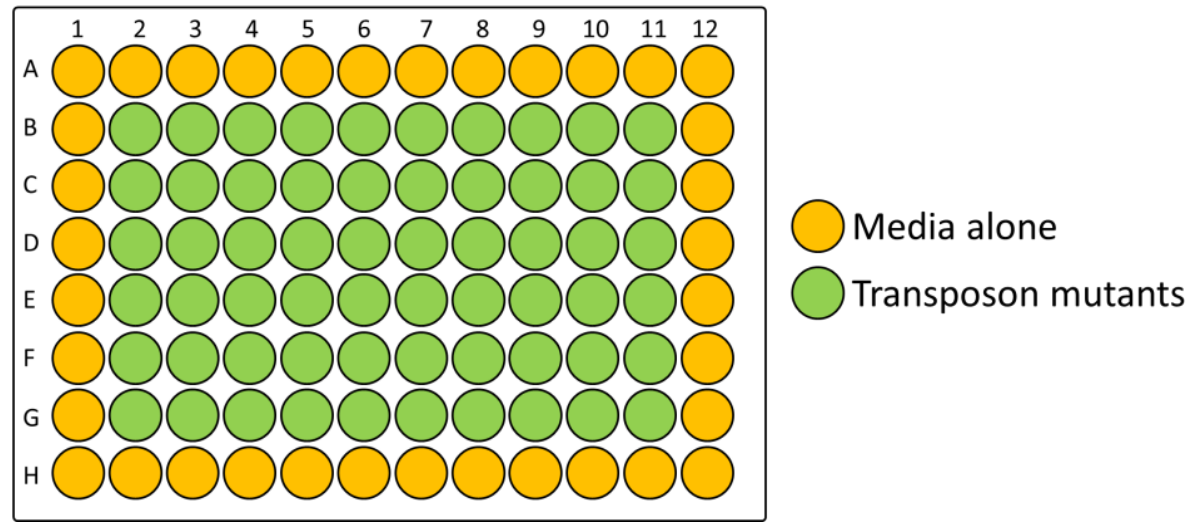

Figure 2.6: Plate setup for secondary assays for drug hypersensitivity. The outermost wells contained media alone (treated or untreated), while the inner 60 wells contained the transposon mutants and media (treated or untreated).

The media alone and media with drug assay plates were run in parallel, sub-inoculated form the same overnight mini-hit library plate. Additionally, two of each media alone and treatment assay plate were run at the same time to serve as biological duplicates. The assay plates were inoculated from the overnight mini-hit library plate using the 96-pin tool before incubation at $41{ }^{\circ} \mathrm{C}$ for 48 hours. At the assay endpoint the $\mathrm{OD}_{600}$ of each well of the assay plates was read using the PerkinElmer EnSpire ${ }^{\circledR}, 2300$ multilable reader. Multiple readings were taken for each well using the three-by-three circular pattern function in the plate-reader software, reading 21 individual points per well, and outputting the average 
$\mathrm{OD}_{600}$ for each well. The experiment was run three independent times for each secondary assay. Assay data was processed as described in section 3.2.4.

\subsubsection{Isoniazid analogue screen}

Isoniazid analogues were tested for their activity against WT $M$. smegmatis $\mathrm{mc}^{2} 155$. To a 96-well plate $100 \mu \mathrm{L}$ of LB was added to all wells. As described in Figure $2.7,100 \mu \mathrm{g} \cdot \mathrm{mL}^{-1}$ of each of the 30 analogues as well as an isoniazid control were added to separate wells. A further $50 \mu \mathrm{L}$ media was added to the outermost wells, and $50 \mu \mathrm{L}$ of a mid-log phase culture of M. smegmatis $\mathrm{mc}^{2} 155$ diluted to an $\mathrm{OD}_{600}$ of 0.05 was added to each of the 60 internal wells.

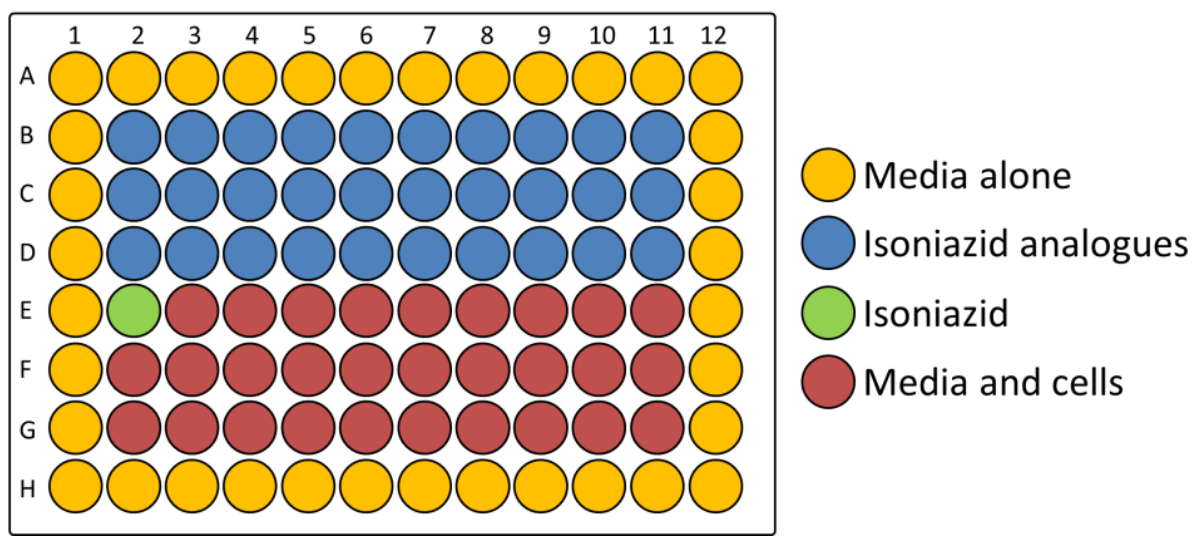

Figure 2.7: Plate layout for isoniazid analogue screen. The outermost wells contained media alone, while the inner 60 wells received $M$. smegmatis cells alone, or cells and drug (isoniazid or isoniazid analogue). Each of the blue wells on the figure received a separate isoniazid analogue.

The assay plate was wrapped in plastic cling film and incubated at $37{ }^{\circ} \mathrm{C}$ for 48 hours. The endpoint $\mathrm{OD}_{600}$ was measured using the PerkinElmer EnSpire ${ }^{\circledR}, 2300$ multilable reader as described in section 2.8.1.2. The $\mathrm{OD}_{600}$ values for each well were normalised by subtracting the median $\mathrm{OD}_{600}$ of the media only wells. The growth percentage was calculated for M. smegmatis in each of the wells containing isoniazid or an isoniazid analogue compared to the median $\mathrm{OD}_{600}$ of the media and cells control wells. 


\subsubsection{Dose-response assay}

Dose-response assays were performed using serially diluted drug to determine drug minimum inhibitory concentration (MIC). The MIC was defined as the minimum concentration of drug to inhibit growth by more than $90 \%$. Dose-response assays were run in 96-well plates with the plate setup as shown in Figure 2.8. Each plate was dived into two halves (dilutions A and B), with separate drugs run in each half for the basic dose-response assays, or the same drug with and without tetracycline for the complementation and overexpression assays Each dilution (A or B) provided three technical replicates at each drug concentration, one replicate per row. The three technical replicates were setup using the same bacterial culture All dose-response assays were setup with mid-log phase cultures at a final $\mathrm{OD}_{600}$ of 0.05 for M. smegmatis $\mathrm{mc}^{2} 155$ or 0.1 for M. tuberculosis $\mathrm{H}_{37} \mathrm{Rv}$.

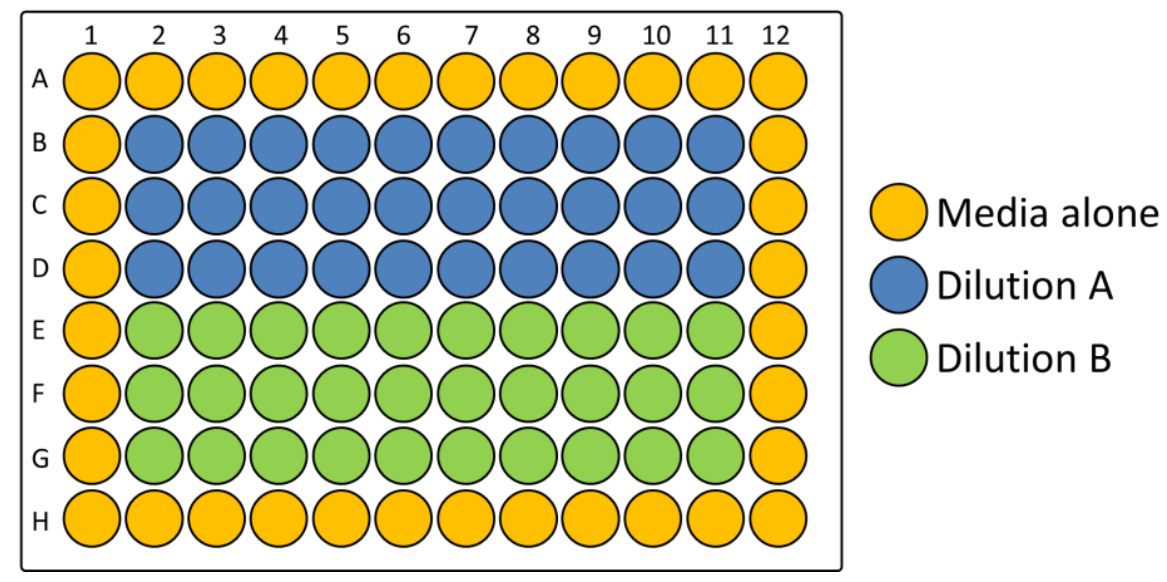

Figure 2.8: Plate layout for dose-response assays. The outermost wells contained media alone, columns $2-10$ contained two-fold serially diluted drug, and column 11 contained no drug and served as the $100 \%$ growth control. Cells were added to all 60 internal wells.

To all internal plate wells except column $2,50 \mu \mathrm{L}$ media was added, and $100 \mu \mathrm{L}$ media added to all external wells. To column $2,100 \mu \mathrm{L}$ media less the volume of drug to be added was aliquoted. The drug was added to column 2 to give a concentration in the column 2 wells at twice the final desired concentration. The drug was then serially diluted across the plate left to right (column 2 - column 10) transferring $50 \mu \mathrm{L}$ from column to column with mixing by pipetting up and down 10 times. After mixing the final dilution in row $10,50 \mu \mathrm{L}$ was drawn into the pipette and discarded. To each of the internal 60 wells, $50 \mu \mathrm{L}$ of cells were then added at a concentration twice the desired final concentration $\left(\mathrm{OD}_{600}\right.$ of 0.05 for 
M. smegmatis or an $\mathrm{OD}_{600}$ of 0.1 for M. tuberculosis). Plates were wrapped with cling film and incubated for 48 hours under the conditions specific for each strain as described in section 2.5.3. Endpoint $\mathrm{OD}_{600}$ was measured with the PerkinElmer EnSpire ${ }^{\circledR}, 2300$ multilable reader as described in section 2.8.1.2. The $\mathrm{OD}_{600}$ values were normalised by subtracting the median $\mathrm{OD}_{600}$ of the media only wells. The $\mathrm{OD}_{600}$ of each drug dilution triplicate was averaged, and compared to the average $\mathrm{OD}_{600}$ of the $100 \%$ growth control triplicate to calculate the growth-inhibition at each drug concentration. MICs were calculated for each separate assay, with experiments repeated three independent times and the mean MIC calculated across the separate assays.

\subsection{Growth curve assay}

Growth curve assays were performed in 96-well plates, using the plate setup described in Figure 2.9. To each of the wells $50 \mu \mathrm{L}$ supplemented LB (section 2.5) was added, with an additional $50 \mu \mathrm{L}$ added to the outside wells. To the internal 60 wells $50 \mu \mathrm{L}$ aliquots of an overnight culture for each strain (section 2.5.3.1), diluted to an $\mathrm{OD}_{600}$ of 0.05 , were added. $\mathrm{A}$ single culture of each strain was used to setup the assay, with each culture aliquoted into multiple wells to provide biological replicates for each strain, enabling an averaging of the growth rates. Plates were wrapped in plastic cling film and incubated at $41{ }^{\circ} \mathrm{C}$ with shaking at $150 \mathrm{rpm}$ for 72 hours total. Every 24 hours, starting at 0 hours, the $\mathrm{OD}_{600}$ of the wells was measured using the PerkinElmer EnSpire ${ }^{\circledR}, 2300$ multilable reader as described in section 2.8.1.2. The median $\mathrm{OD}_{600}$ of the on plate biological replicates for each strain were averaged for each time point. The assay was run three independent times, with the mean $\mathrm{OD}_{600}$ of each strain averaged across the three assays for each time point. 


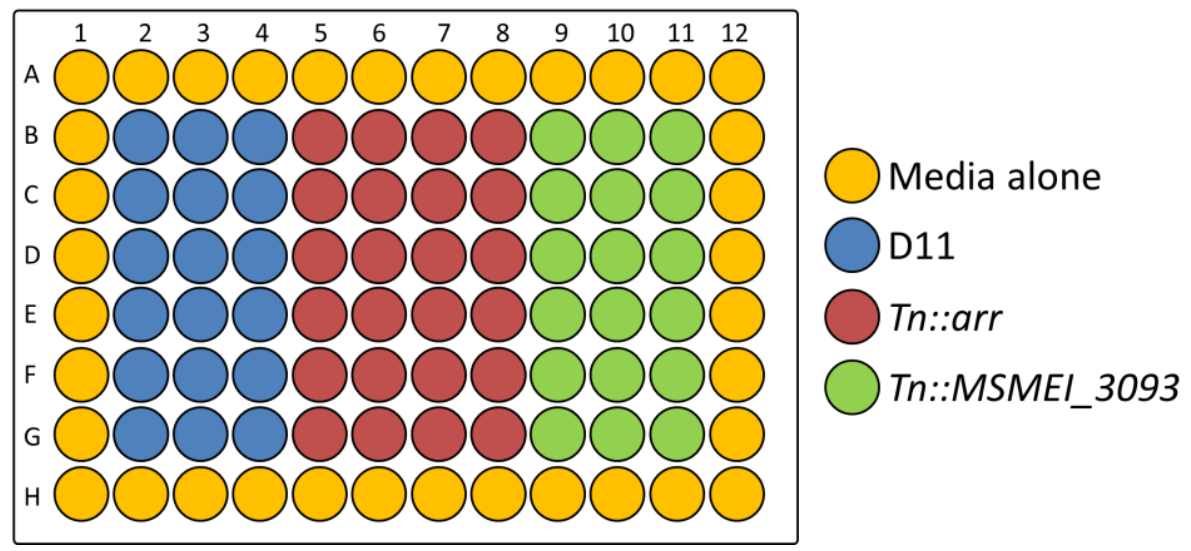

Figure 2.9: Growth curve assay plate setup. The outermost wells contained media alone. The inner 60 wells were divided between the three strains tested, D11, Tn::arr, and Tn::MSMEI_3093. 


\section{Isolation of drug hypersensitive mutants of $M$. smegmatis mc ${ }^{2} 155$}

\subsection{Introduction}

To date, little work has been done on studying drug sensitising mutations for the anti-mycobacterial drugs isoniazid, rifampicin, and bedaquiline, with most research instead focused on studying resistance inducing mutations and altered transcription upon drug exposure. As drug hypersensitivity is a recessive phenotype it cannot be selected for in the same manner as resistant mutants, i.e. in a heterogeneous culture, as sensitive mutants will fail to grow and be lost. This limitation is overcome by the prior construction of a collection of mutants that are then assessed for sensitivity independently. Different methods for the generation of mycobacterial mutant collections for phenotypic screening exist, with transposon mutagenesis favoured due to the inherent labelling of the site of the mutation by the transposon insertion.

\subsubsection{Transposon mutagenesis with Tn611}

Tn611 is based on the Tn610 transposon, which has two inverted IS6100 insertion elements flanking a sulphonamide resistance gene sul3, replaced with a gene for kanamycin resistance (aph) to generate Tn611. Insertion of Tn611 into a vector that contained both a temperature sensitive pAL5000 mycobacterial origin of replication and an E. coli origin (pUC) of replication generated a plasmid named pCG79 (Figure 3.1). This transposon has been shown to insert into the genome of $M$. smegmatis randomly with no insertional hotspots detected (BillmanJacobe et al., 2006; Guilhot et al., 1994); additionally, insertion is a rare event with only one insertion per cell being reported (Billman-Jacobe et al., 2006; Guilhot et al., 1994). Insertion into the mycobacterial genome occurs by a "copy and paste" mechanism resulting in duplication of one of the insertion elements (Martin et al., 1990), although other possible insertional methods have been reported such as a "cut and paste" mechanism (Guilhot et al., 1994). Insertion of the Tn611 transposon into the genome has been shown to be stable in the absence of any selective pressure (Guilhot et al., 1994). However, the stability of the insertion under selective pressure is not known. 


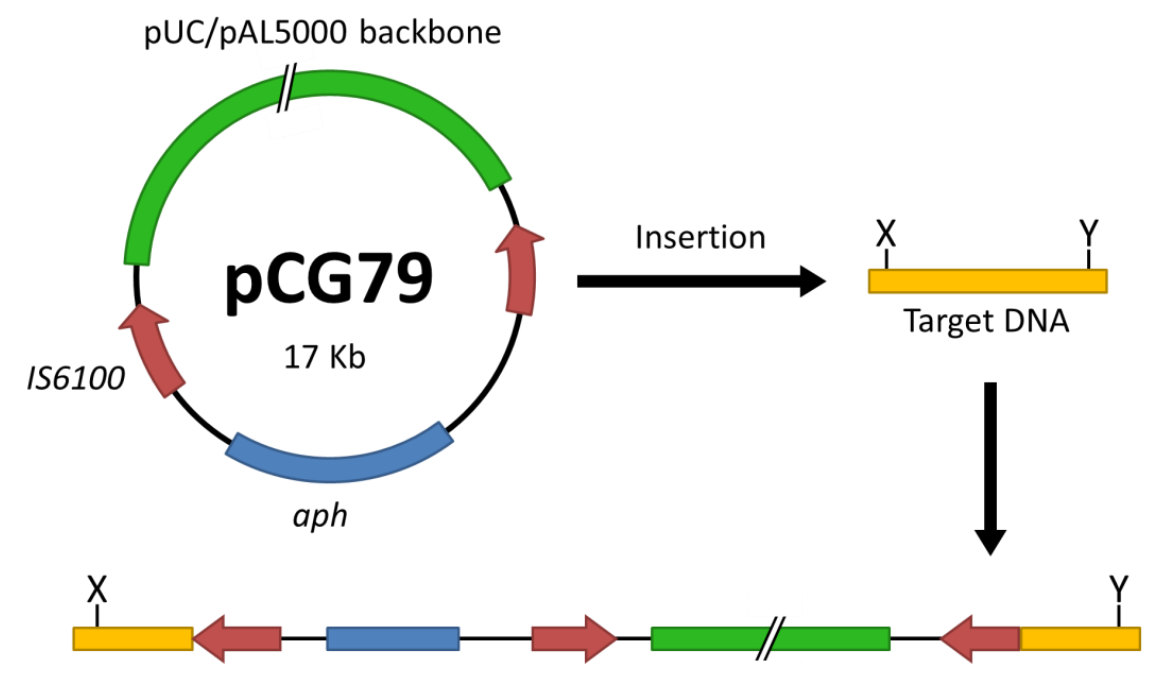

Figure 3.1: Plasmid pCG79 and transposition. Insertion of Tn611 into the target DNA via a replicative mechanism results in duplication of one of the two 156100 elements and insertion of the entire pCG79 vector.

\subsubsection{Assaying for drug hypersensitivity}

Growth-inhibition is commonly measured to assess the inhibitory effect of different conditions in bacteria. A number of techniques are used to measure growth, and therefore growth-inhibition, with direct counting of colony forming units (CFUs) being the gold standard as it is the most direct observation of cell number. However, $\mathrm{OD}_{600}$ is commonly used as a surrogate for CFU measurements as CFU measurements are impractical for higher throughput analyses. Recently, resazurin, a blue weakly fluorescent dye, has been utilised as another alternate method to CFUs for measuring the growth of mycobacteria (Palomino and Portaels, 1999). Resazurin is reduced to the pink, highly fluorescent product resorufin in the presence of NADH, and is used as a measure of oxidative phosphorylation. The dye is commonly used as a viability indicator as only living cells will produce the required NADH, however, recent work has demonstrated a strong correlation between the reduction of resazurin and CFUs for measuring the growth of logarithmic cultures of $M$. smegmatis (Bassett et al., 2013). Measurement of resazurin reduction as an indicator of growth in micro-titre plates is much more suitable for high throughput analyses of mycobacteria than the more direct measure of growth OD. This is because resazurin, and the reduced resorufin, diffuse easily through a liquid medium resulting in a uniform fluorescence and therefore instil a requirement of only one measurement per well. In contrast, OD based measurements 
of mycobacteria in micro-titre plates require multiple readings per well to generate reliable measurements of cell growth, owing to the characteristic cell clumping. However, unlike CFU and OD based measurements, the fluorescence generated from the reduction of resazurin is a relative measure of growth, with longer incubation times resulting in different absolute fluorescence values. For the purposes of screening a transposon mutant collection, the inclusion of a standard strain that acts as an untreated (100\% growth) control in each library plate allows standardisation of the growth-inhibition as measured by resazurin reduction, enabling the direct comparison between separate library plates. The reduction of resazurin is also affected by the metabolic states of the cultures which, similar to other indirect measure of cell number, can lead to false positives (Pethe et al., 2010). This limitation can be overcome by using a secondary assay to validate the drug hypersensitivity of mutants using an endpoint OD measurement. Although using OD results in a lower throughput assay, using it as a validation assay for a limited number of the most sensitive mutants from the primary assay overcomes this limitation.

\subsubsection{Objectives of Chapter Three}

The goal of Chapter Three was to generate drug hypersensitive mutants of $M$. smegmatis $m c^{2} 155$. These mutants were then studied to identify the underlying genetic basis and the information used to investigate drug mode of action in Chapters Four and Five of this thesis

To achieve this goal the following specific aims were addressed:

- Construction of a random transposon mutant collection in M. smegmatis $\mathrm{mc}^{2} 155$ using Tn611

- Development of a high-throughput assay for identifying drug hypersensitive mutants

- Development of a secondary assay to validate mutants identified as drug hypersensitive in the primary assay

Drug hypersensitive mutants were generated in order to identify novel components of the mode of action of the front line anti-TB drugs isoniazid, rifampicin, and bedaquiline. $A$ collection of $M$. smegmatis $\mathrm{mc}^{2} 155$ transposon mutants were generated in order to assay for increased drug hypersensitivity and identify mutant strains of interest. Sensitivity of these mutants was ultimately characterised in terms of MICs. 


\subsection{Methods}

\subsubsection{Estimation of unique mutants versus library size}

The expected number of unique mutants in a random transposon mutant collection was estimated for a range of collection sizes using the Poisson distribution (Equation 3.1 and Equation 3.2), and the following assumptions:

- The percentage of essential genes in $M$. smegmatis $\mathrm{mc}^{2} 155$ is the same as that previously described for M. tuberculosis (Sassetti et al., 2001)

- The Tn611 transposon inserts into the genome of M. smegmatis in a random manner, i.e. lacks insertional hot spots (Guilhot et al., 1994)

- The probability of the transposon inserting into an open reading frame (ORF) is identical for all ORFs regardless of their size

$$
P(x)=\frac{e^{-\frac{L}{G}} \cdot(L / G)}{x}
$$

Equation 3.1: Poisson estimation of the standard distribution. Where $L$ is equal to the total number of mutants in the library, and $G$ is equal to the number of genes in the $M$. smegmatis genome that the transposon can insert into.

$$
m=G\left(1-e^{-\frac{L}{G}}\right)
$$

Equation 3.2: Calculation for the estimated number of unique mutants in a library of size L. For a given library size $(L)$ and based upon the above assumptions the number of unique mutants is estimated to be equal to $m$.

$G$ was estimated based on the percentage of non-essential genes in $M$. tuberculosis $\mathrm{H}_{37} \mathrm{Rv}$, i.e. $86.3 \%$ of its genome; $G$ is therefore equal to $86.3 \%$ of the 6752 genes in the M. smegmatis $\mathrm{mc}^{2} 155$ genome or 5827 genes. It was determined that a library size of approximately 7680 transposon mutants, which equates to approximately 1.1-fold whole genome coverage, would be suitable for screening for drug hypersensitivity. At this library size it is expected to contain 4267 unique mutants, interrupting $73.2 \%$ of the genes predicted to be able to sustain transposon insertions (non-essential genes) (Figure 3.2). In order to increase the proportion of genes knocked out, a significant increase in the number 
of mutants would be required. For example, 20,000 or 60,000 mutants would be required to obtain $95 \%$ or $100 \%$ of genes knocked out, respectively. The aim of this thesis was to identify pathways related to drug mode of action, as opposed to a single gene whose product was affected by a drug and therefore, it was not critical to have every possible gene knocked out within this library. Additionally, the smaller size of the library constructed in this thesis facilitates screening in high-throughput. Libraries with 1.1-fold genome coverage have been used previously for similar studies (Alexander et al., 2003; Daugherty et al., 2011).

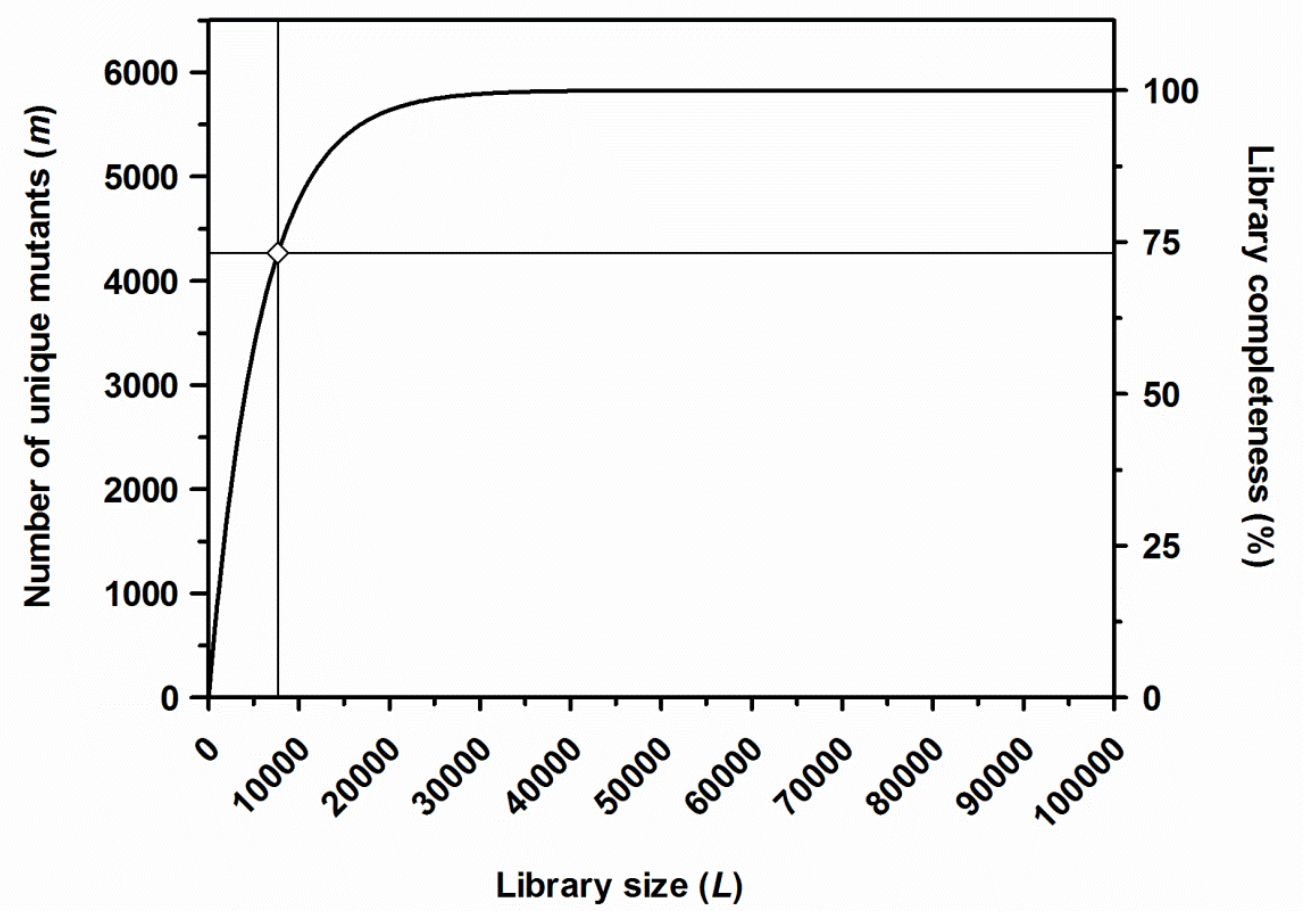

Figure 3.2: Number of unique mutants versus library size. Line graph describing the expected number of unique mutants $(m)$ for a given library size $(L)$ estimated using the Poisson distribution. Library completeness is the number of expected mutants $(m)$ as a percentage of the number of the predicted non-essential genes for M. smegmatis. Line intersect indicates chosen library size of 7680 transposon mutants.

\subsubsection{Construction of the transposon mutant collection}

A collection of 7680 transposon mutants was built in 32 384-well plates, with 240 individual transposon mutants per plate as described in section 2.7. As discussed in the chapter introduction, the fluorescence from resazurin reduction generates a relative measure of growth-inhibition for that plate. In order to compare the fluorescence values and therefore 
growth-inhibition of mutants across multiple plates a standard was included in each plate of the library. The selected standard was an untreated control strain (MRC10) that would represent maximal growth (maximal resazurin reduction) for that plate, against which the resazurin reduction of the mutant strains on that plate were standardised. To compare growth-inhibition of mutants across separate plates of a library it was essential the treatment conditions were the same. To test this, half of the MRC10 control strain wells remained untreated, while the other half were treated with the same drug concentration as the transposon mutants, with statistical testing being used to check that the treated-MRC10 control behaved the same across all plates of a library.

\subsubsection{Primary assay for drug hypersensitivity}

The assays for identifying drug hypersensitive mutants were performed using drug concentrations that gave minor but measureable growth-inhibition, with screening concentrations for each drug optimised as described below in section 3.2.3.1. At these concentrations mutants that displayed hypersensitivity to a drug could be differentiated from the average growth-inhibition levels of the mutant libraries. Drugs were screened against the transposon mutant libraries as described in section 2.8.1. For logistical reasons the 32 plate transposon mutant collection was split into two smaller 16 plate libraries for the purpose of performing the primary assay, Library A and Library B. The data analysis for the primary assays against each library were performed independently to prevent any bias resulting from different assay conditions. To quantify drug sensitivity, a factor of inhibition $\left(F_{I}\right)$ was calculated for each mutant strain for each drug. This value is the reciprocal of the growth of the strain when treated versus untreated, with higher values representing increased inhibition in the assay (Equation 3.4). The output from the primary assay was a list of mutants ordered from highest to lowest sensitivity to the drug used.

To calculate the $F_{1}$, the relative fluorescence values from the reduction of resazurin were normalised by removing the background, giving the relative fluorescence units (RFU) for each well of the plate. The in-plate untreated-MRC10 control, which represented maximal resazurin reduction (therefore maximal growth), was averaged to give RFU UTMRC10 that was 
used to standardise the RFU values of the treated-MRC10 control and mutant strains across the rest of the plate using Equation 3.3.

$$
\mathrm{sRFU}_{\text {myco }}=100 \cdot\left(\frac{\mathrm{RFU}_{\text {myco }}}{\mathrm{RFU}_{\text {UTMRC10 }}}\right)
$$

Equation 3.3: Calculation of standardised RFU values for transposon mutants. Where $R_{F U}$ myco is the normalised RFU and $\mathrm{SRFU} \mathrm{myco}_{\text {is }}$ the standardised RFU for a given strain.

The $F_{1}$ for each mutant strain (and treated-MRC10 control) was calculated according to Equation 3.4. An $F_{1}$ value of 1 represented no inhibition while a value greater than 1 represented an increased inhibition, with the greater the $F_{\text {I }}$ value the greater the strain was inhibited.

$$
F_{I}=\frac{1}{\left(\frac{\mu_{\text {treated }}}{\mu_{\text {untreated }}}\right)}
$$

Equation 3.4: Calculation of $F_{1}$ for mutants. Where $\mu_{\text {treated }}$ and $\mu_{\text {untreated }}$ are the mean $s R F U_{\text {myco }}$ values with and without drug treatment respectively. Means were calculated over three independent experiments.

The on plate treated-MRC10 control was used to test that all plates for a given condition were behaving the same throughout all experimental replicates. The median SRFU values of the treated-MRC10 from each plate of a library were pooled and tested for normal distribution (D'agostino and Pearson omnibus normality test), with data normalised as required using transformations listed in the results sections for each drug. A one-way ANOVA was performed to test if there was a significant difference in the average inhibition of the treated-MRC10 control strain across all plates of a mutant library before directly comparing the growth-inhibition of the mutants to determine the 60 most sensitive. The 60 most sensitive mutants from each library (Library A and Library B), for each drug, were combined into mini-hit libraries as described in section 2.7.3.

\subsubsection{Optimising screening concentrations}

Two separate plates from the transposon mutant libraries, selected at random, were grown with and without drug to optimise drug screening concentrations. A range of concentrations 
of drug were used, and these were selected by determining the MIC versus MRC10 as a starting reference point, with MICs determined by dose-response assay described in section 2.8.3. The median $F_{1}$ for each different drug concentration test plate was determined by averaging the $F_{1}$ values for all mutants within a given condition. A drug concentration that gave a median $F_{\mid}$value in the range of 1.18 - 1.42 was selected as the screening concentration for that drug. This $F_{1}$ range represents a median inhibition of $15-30 \%$.

\subsubsection{Secondary assay for drug hypersensitivity}

The secondary assay was run to reduce the number of false positives resulting from the use of resazurin reduction as a measure of growth, instead using the more biologically relevant measure of growth, $O D$ at $600 \mathrm{~nm}\left(O D_{600}\right)$ as discussed in the introduction to this chapter. The mini-hit libraries comprised of the 60 most sensitive mutants for a give drug from each library (120 total), and were expected to capture most if not all the true positive hits from the initial screen, while minimising the number of mutants for validation. These mini-hit libraries were screened as described in 2.8.1.2. A plate containing only the strain MRC10 was also run against each drug for comparison.

\subsection{Results}

\subsubsection{Drug screening concentration optimisation}

The two frontline anti-TB drugs isoniazid and rifampicin, and the novel anti-mycobacterial bedaquiline, were assayed against the transposon mutant libraries to find drug hypersensitive mutants. The MIC concentrations for these compounds against the control strain MRC10, and the screening concentrations used are listed in Table 3.1. Screening concentrations were chosen as described in section 3.2.3.1. Work on bedaquiline was performed in collaboration with the laboratory of Professor Gregory Cook at the University of Otago. 
Table 3.1: MIC values for anti-TB drugs versus MRC10 and optimised screening concentrations.

\begin{tabular}{ccc}
\hline Drug & MIC $^{\mathrm{a}} \pm$ se & $\begin{array}{c}\text { Screening } \\
\text { concentration }\end{array}$ \\
\hline Isoniazid & $6.12 \pm 0.56 \mu \mathrm{M}$ & $6.00 \mu \mathrm{M}$ \\
Rifampicin & $0.72 \pm 0.12 \mu \mathrm{M}$ & $0.26 \mu \mathrm{M}$ \\
Bedaquiline & $29.3 \pm 7.32 \mathrm{nM}$ & $10.8 \mathrm{nM}$ \\
\hline
\end{tabular}

${ }^{\mathrm{a}} \mathrm{MIC}$ values represent mean values across three independent experiments with standard error (se) presented.

\subsubsection{Growth of the treated-MRC10 control strain in LB}

The growth of the treated-MRC10 controls were examined for variability between plates within each library when grown in media (LB) alone. The SRFU values of the treated-MRC10 control strain were normally distributed in both libraries, and were examined using a one-way ANOVA. No significant difference between the SRFU values of the treated-MRC10 control strain across the separate plates from the three independent experimental replicates was found for either library; therefore, screening for hypersensitive transposon mutants proceeded.

\subsubsection{Control strains for quantitating drug hypersensitivity}

Ten mutant strains were randomly selected from the mutant libraries to act as control strains for the dose-response assays. The MICs of these control strains for isoniazid, rifampicin, and bedaquiline were determined (Figure 3.3). The MIC values of the control strains used were consistent for each of the drugs tested (Kruskal-Wallis test; significance cut off of $p<0.05)$. The mean of all 10 control strains was used for the initial dose-response assays to quantify drug hypersensitivity. 
A

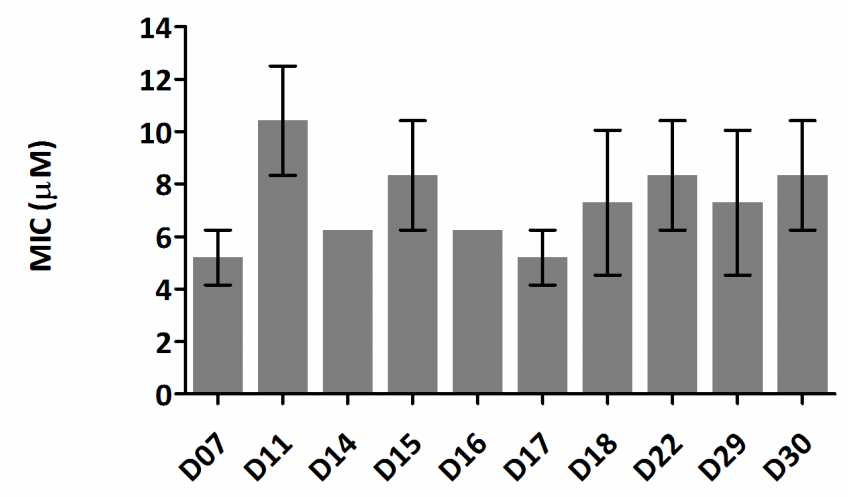

B

Mutant Strain

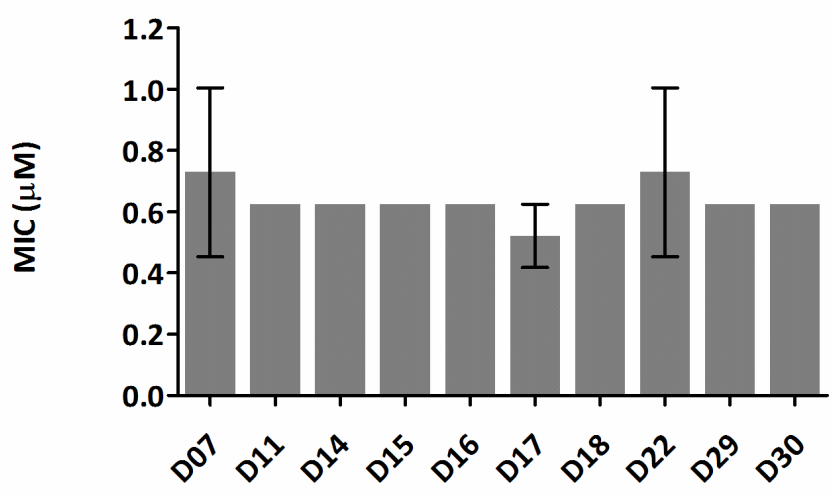

C

Mutant Strain

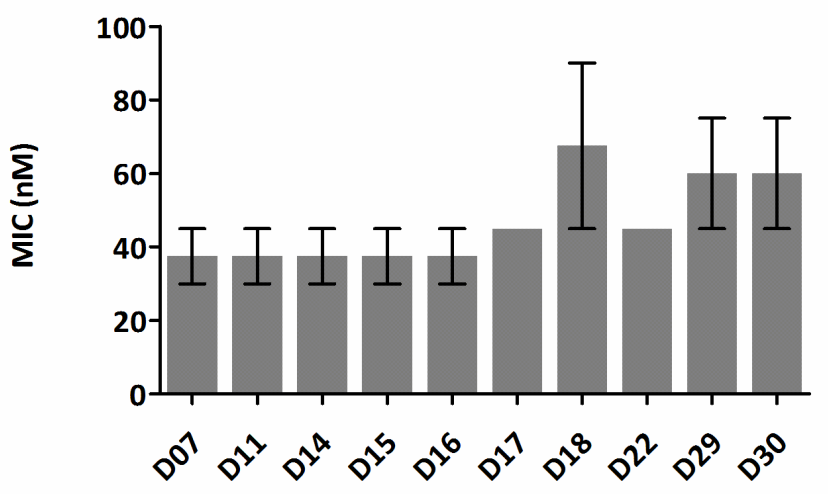

Mutant Strain

Figure 3.3: Comparison of MIC of random mutant control strains. Average MIC values of 10 random control transposon mutant strains versus (A) isoniazid, (B) rifampicin, and (C) bedaquiline. Mutants are labelled according to the mutant library plate they were picked from. Column heights represent mean MICs across three independent experiments with standard error bars shown. 


\subsubsection{Isoniazid}

\subsubsection{Primary assay for drug hypersensitivity}

The primary assay for isoniazid hypersensitivity was performed at a concentration of $6 \mu \mathrm{M}$ (Table 3.1). As the sRFU values for the treated-MRC10 controls within each library were normally distributed one-way ANOVAs were performed on untransformed data. The one-way ANOVAs did not show a significant difference in mean sRFU for the treated-MRC10 control across all plates within either library (A or B) $(p>0.05)$, therefore analysis of the assay data continued as described in section 3.2.3.

The median inhibition of mutant library A versus isoniazid was approximately $19 \%$ (Figure 3.4). The 60 most sensitive mutants from library A, representing mutants that were at least 1.9 times more sensitive to isoniazid than the library median, were picked into the isoniazid mini-hit library. 

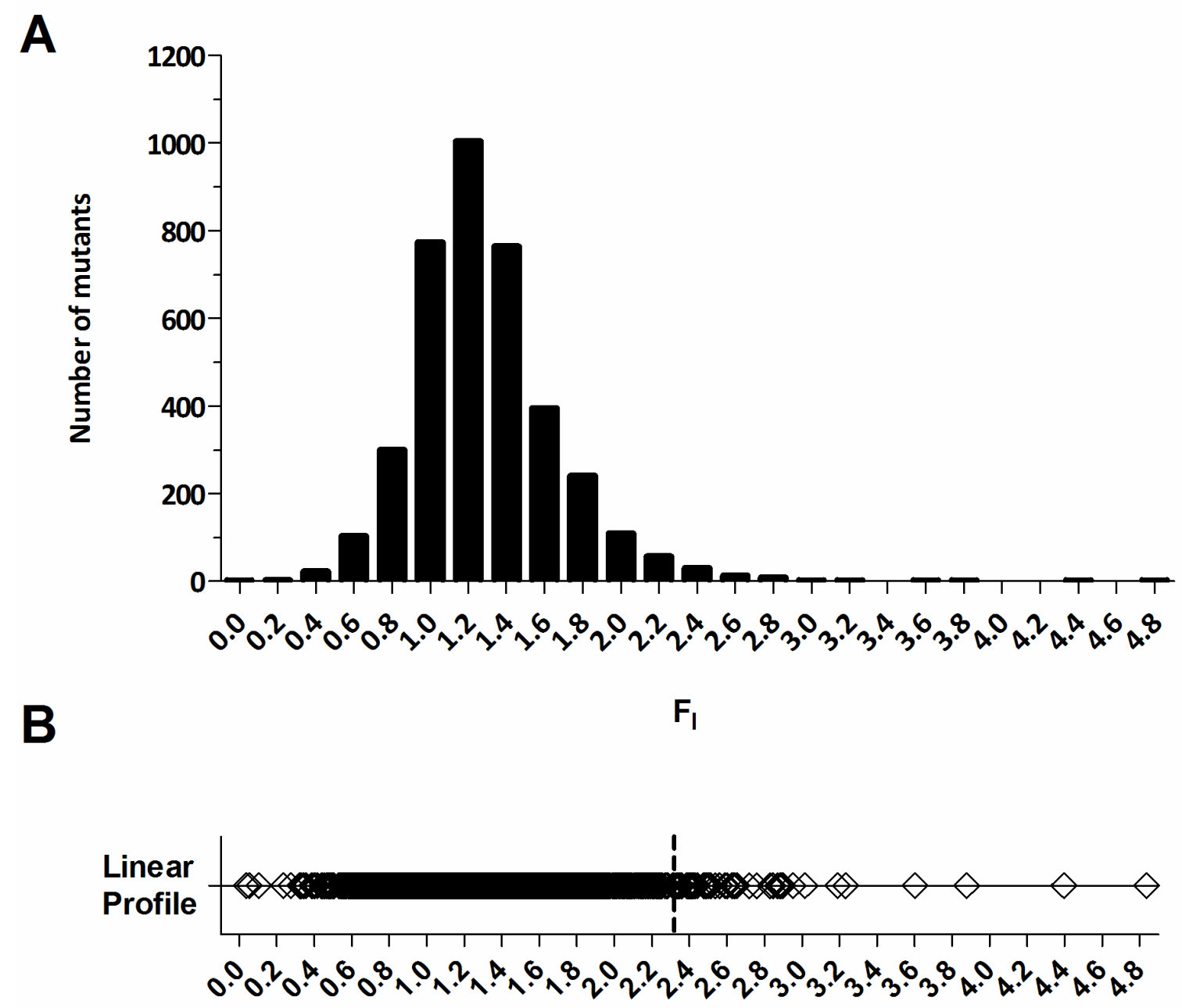

$\mathbf{F}_{\mathbf{I}}$

Figure 3.4: Mutant library A versus $6 \mu \mathrm{M}$ isoniazid. (A) Frequency distribution of $F_{1}$ values, and (B) $F_{1}$ linear profile, from library A versus $6 \mu \mathrm{M}$ isoniazid. The median $F_{1}$ across mutant library A was 1.21, and the median $F_{1}$ for the treated-MRC10 control was 1.26. The dotted line indicates the cut-off for the 60 most sensitive mutants, and lies at $F_{1}=2.30$. The $F_{1}$ value for each mutant represents the mean $F_{I}$ across three independent experiments.

The median inhibition of mutant library B versus isoniazid was approximately $13 \%$ (Figure 3.5). The 60 most sensitive mutants from library $B$, representing mutants that were at least 2.0 times more sensitive to isoniazid than the library median, were picked into the isoniazid mini-hit library. 


\section{A}
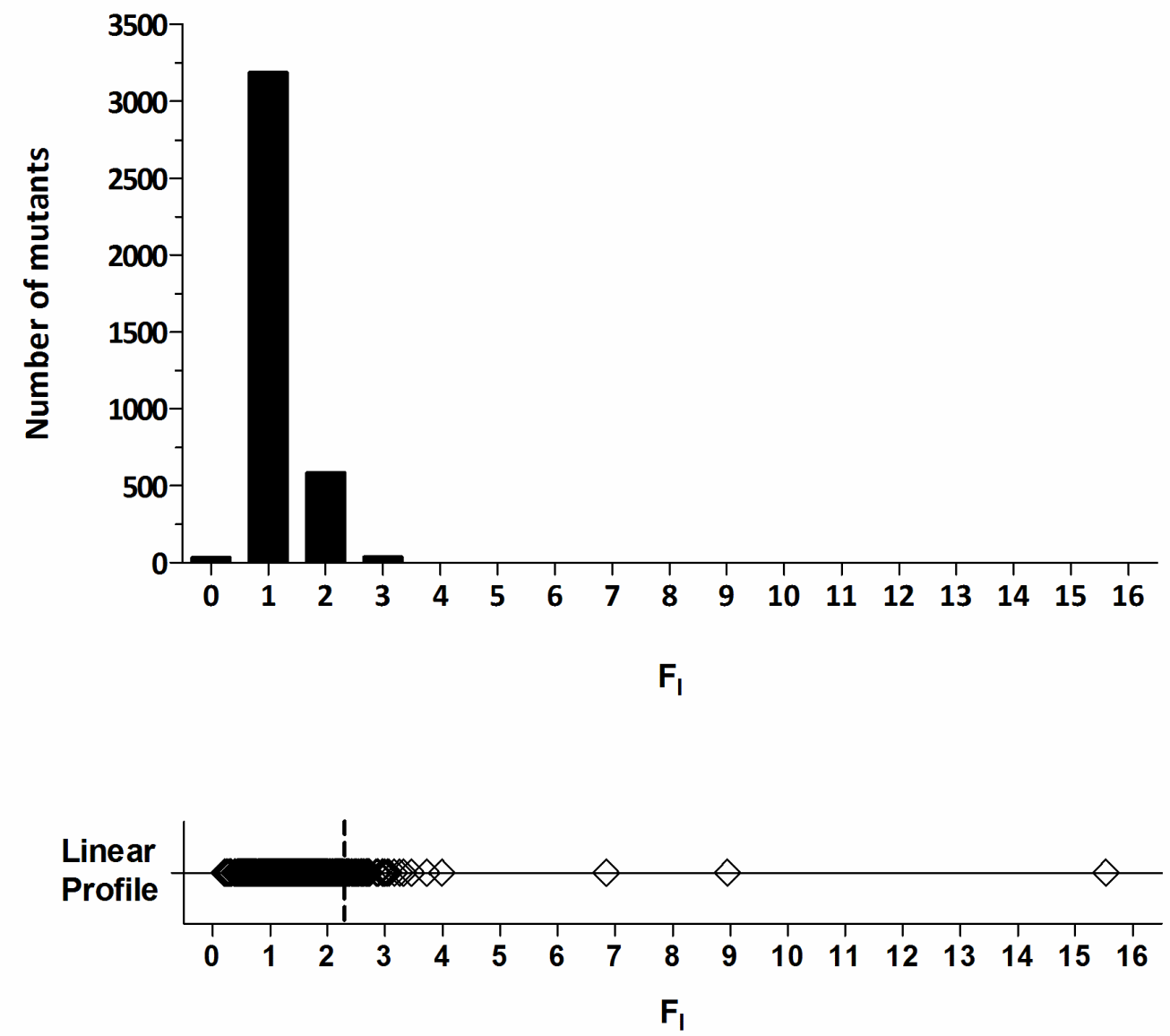

Figure 3.5: Mutant library B versus $6 \mu \mathrm{M}$ isoniazid. (A) Frequency distribution of $F_{1}$ values, and (B) $F_{1}$ linear profile, from library B versus $6 \mu \mathrm{M}$ isoniazid. The median $F_{I}$ across mutant library B was 1.15, and the median $F_{1}$ for the treated-MRC10 control was 1.19. The dotted line indicates the cut-off for the 60 most sensitive mutants, and lies at $F_{1}=2.29$. The $F_{1}$ value for each mutant represents the mean $F_{l}$ across three independent experiments.

\subsubsection{Secondary assay for drug hypersensitivity}

A secondary assay was performed in order to validate the hypersensitivity to isoniazid of the 120 most sensitive mutants (60 from each library) identified in the primary assay. Each hypersensitive mutant was tested against the screening concentration of isoniazid (Table 3.1) and growth-inhibition was determined by endpoint $O D_{600}$ measurement as described in section 3.2.4. The concentration of isoniazid used in the secondary assay was the same as that used in the primary assay $(6 \mu \mathrm{M})$. Table 3.2 shows the 12 most sensitive mutants from 
the secondary assay against the isoniazid mini-hit library. The growth-inhibition of mutants in the secondary assay against the isoniazid mini-hit library showed a median growth-inhibition of $23.0 \%$, similar to the growth-inhibition of the MRC10 control strain at 23.6\%. Three mutants, myco7437, myco2576, and myco5016 stood out, showing greater than $80 \%$ inhibition.

Table 3.2: Growth-inhibition of top secondary assay hits versus isoniazid.

\begin{tabular}{cc}
\hline Strain & Growth-inhibition $^{\mathrm{a}} \pm$ se (\%) \\
\hline myco7437 & $97.9 \pm 0.84$ \\
myco2576 & $87.3 \pm 9.29$ \\
myco5016 & $85.5 \pm 5.91$ \\
$m y c 03113$ & $61.9 \pm 19.8$ \\
myco0409 & $54.3 \pm 14.3$ \\
myco6166 & $54.2 \pm 12.4$ \\
myco2100 & $49.8 \pm 13.2$ \\
$m y c 01897$ & $49.6 \pm 15.6$ \\
myco6192 & $48.7 \pm 18.5$ \\
myco6482 & $46.5 \pm 12.0$ \\
myco2659 & $45.5 \pm 2.78$ \\
myco7102 & $45.2 \pm 12.6$ \\
MRC10 & $23.6 \pm 5.48$
\end{tabular}

${ }^{\mathrm{a}}$ Growth-inhibition values represent mean values across three independent assays with standard errors (se) presented.

\subsubsection{Quantification of mutant hypersensitivity}

The isoniazid MIC was determined by dose-response assay (section 2.8.3) for the 12 most sensitive mutants from the secondary assay in order to quantify mutant sensitivity. Isoniazid MICs for the 12 strains were compared to an MIC value averaged across the 10 random transposon mutant control strains (section 3.3.3). All of the 12 isoniazid hypersensitive mutants from the secondary assay showed some level of increased sensitivity compared to the control MIC (Table 3.3). The three mutants that stood out in the secondary assay also showed the greatest change in isoniazid MIC. These mutants, myco2576, myco5016, and myco7437, showed a greatly increased sensitivity to isoniazid with a decrease in MIC of greater than two-fold compared to the control MIC, with myco2576 and myco5016 showing a greater than four-fold decrease in MIC. 
Table 3.3: Sensitivity of transposon mutants to isoniazid.

\begin{tabular}{cc}
\hline Strain & MIC \pm se $(\boldsymbol{\mu M})$ \\
\hline myco2576 & $1.56 \pm 0.00$ \\
myco5016 & $1.56 \pm 0.00$ \\
myco7437 & $3.12 \pm 0.00$ \\
myco2100 & $4.17 \pm 1.04$ \\
myco3113 & $5.21 \pm 1.04$ \\
myco0409 & $5.21 \pm 1.04$ \\
myco6166 & $5.21 \pm 1.04$ \\
myco1897 & $6.25 \pm 0.00$ \\
myco6192 & $6.25 \pm 0.00$ \\
myco6482 & $6.25 \pm 0.00$ \\
myco2659 & $6.25 \pm 0.00$ \\
myco7102 & $6.25 \pm 0.00$ \\
Tn Control & $7.08 \pm 0.54$ \\
\hline
\end{tabular}

Isoniazid MICs for the 12 mutants most sensitive to isoniazid identified in the secondary assay, averaged over three independent experiments. The transposon $(\mathrm{Tn})$ control value represents the mean of the 10 randomly selected mutant control strains. Standard errors (se) are presented.

\subsubsection{Rifampicin}

\subsubsection{Primary assay for drug hypersensitivity}

The primary assay for rifampicin hypersensitivity was performed at a concentration of $0.26 \mu \mathrm{M}$ (Table 3.1). As the SRFU values for the treated-MRC10 controls within each library were normally distributed one-way ANOVAs were performed on untransformed data. The one-way ANOVAs did not show a significant difference in mean SRFU for the treated-MRC10 control across all plates of either library A or B $(p>0.05)$, therefore analysis of the assay data continued as described in section 3.2.3.

The median inhibition of mutant library A versus rifampicin was approximately $24 \%$ (Figure 3.6). The 60 most sensitive mutants from library $A$, representing mutants that were at least 1.78 times more sensitive to rifampicin than the library median, were picked into the rifampicin mini-hit library. 
A

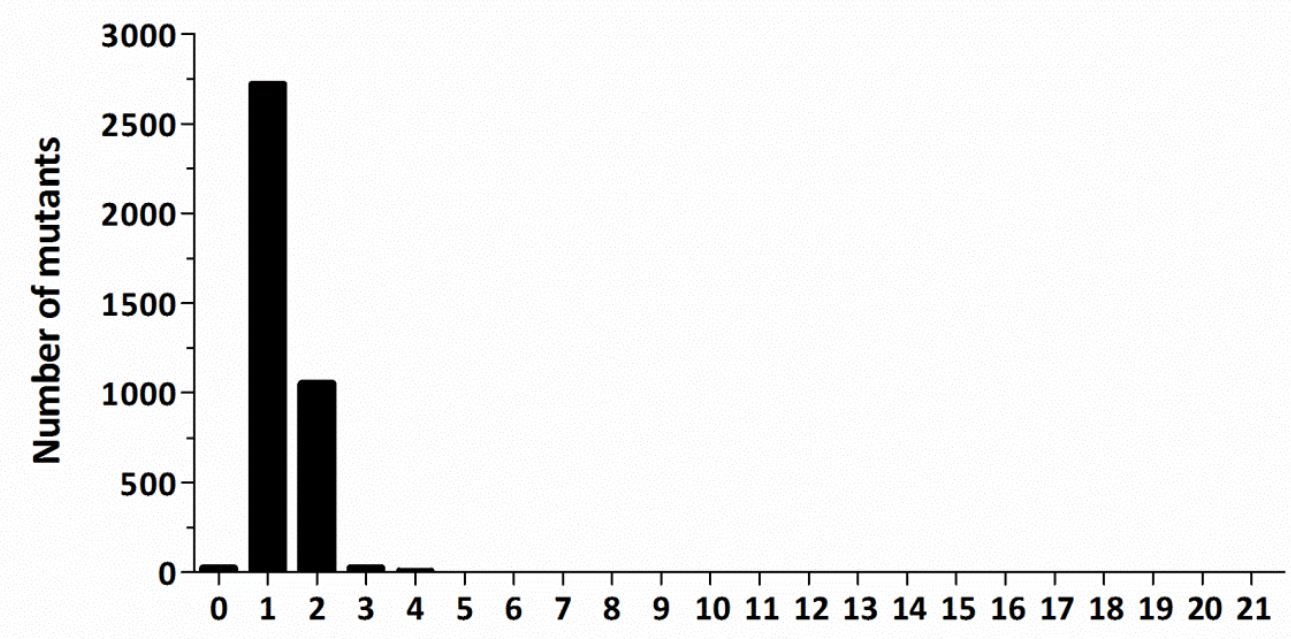

B

$F_{1}$

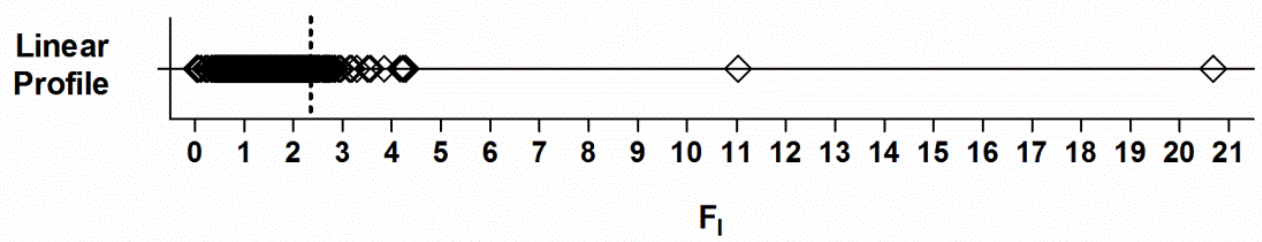

Figure 3.6: Mutant library A versus $0.26 \mu \mathrm{M}$ rifampicin. (A) Frequency distribution of $F_{1}$ values, and (B) $F_{1}$ linear profile, from library A versus $0.26 \mu \mathrm{M}$ rifampicin. The median $F_{I}$ across mutant library A was 1.32 , and the median $F_{1}$ for the treated-MRC10 control was 1.60 . The dotted line indicates the cut-off for the 60 most sensitive mutants, and lies at $F_{1}=2.35$. The $F_{1}$ value for each mutant represents the mean $\mathrm{F}_{1}$ across three independent experiments.

The median inhibition of mutant library B versus rifampicin was approximately $5 \%$ (Figure 3.7). The 60 most sensitive mutants from library $B$, representing mutants that were at least 1.72 times more sensitive to rifampicin than the library median, were picked into the rifampicin mini-hit library. 

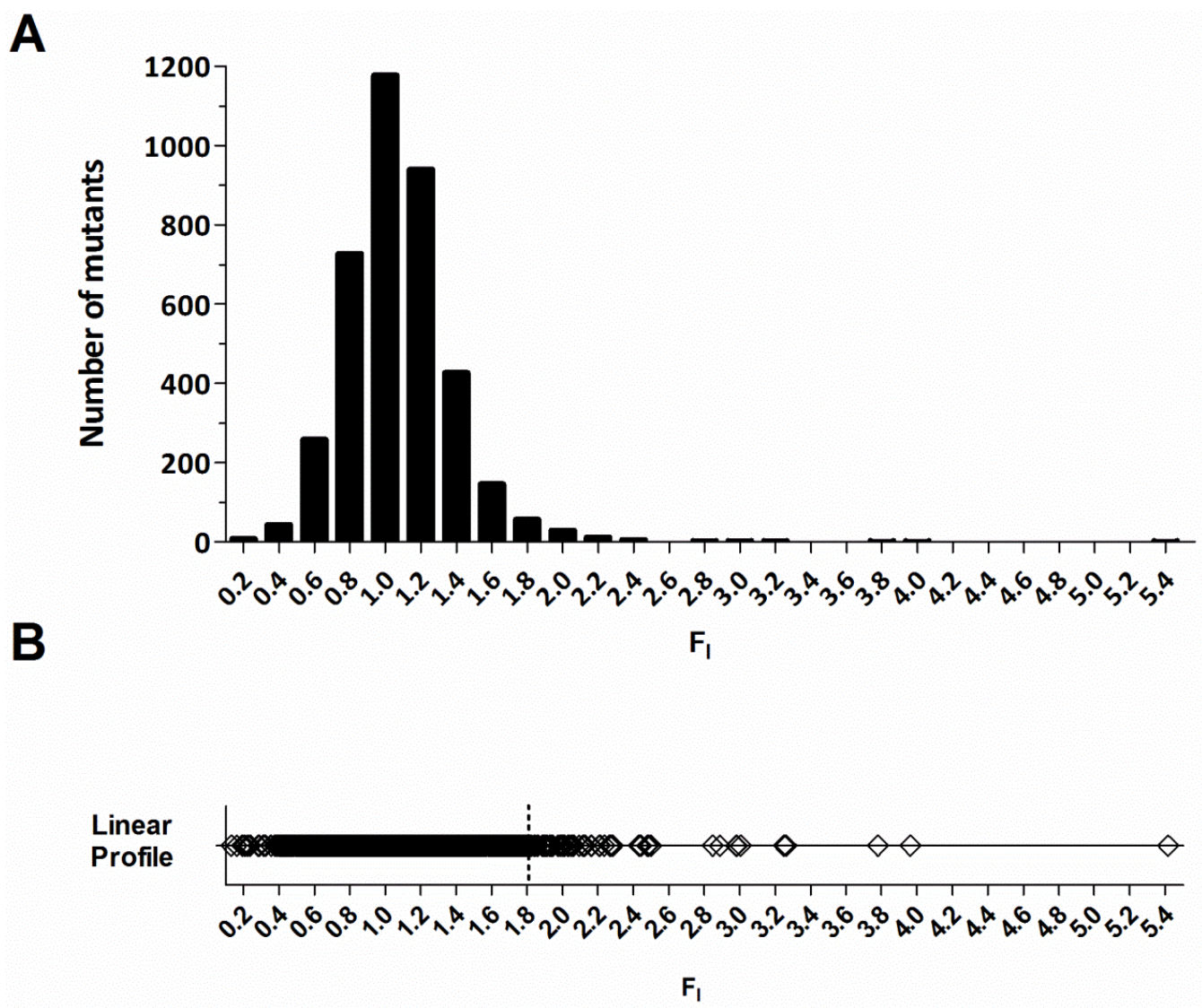

Figure 3.7: Mutant library $B$ versus $0.26 \mu M$ rifampicin. (A) Frequency distribution of $F_{1}$ values, and (B) $F_{I}$ linear profile, from library B versus $0.26 \mu \mathrm{M}$ rifampicin. The median $F_{I}$ across mutant library $B$ was 1.05 , and the median $F_{1}$ for the treated-MRC10 control was 1.36 . The dotted line indicates the cut-off for the 120 most sensitive mutants, and lies at $F_{1}=1.81$. The $F_{1}$ value for each mutant represents the mean $\mathrm{F}_{1}$ across three independent experiments.

\subsubsection{Secondary assay for drug hypersensitivity}

A secondary assay was performed In order to validate the hypersensitivity to rifampicin of the 120 most sensitive mutants (60 from each library) identified in the primary assay. Each hypersensitive mutant was tested against the screening concentration of rifampicin (Table 3.1) and growth-inhibition was determined by endpoint $O D_{600}$ measurement as described in section 3.2.4. The concentration of rifampicin used in the secondary assay was the same as that used in the primary assay $(0.26 \mu \mathrm{M})$. Table 3.4 shows the 12 most sensitive mutants from the secondary assay against the rifampicin mini-hit library. The median growth-inhibition of the entire mini-hit library was $8.46 \%$, compared to $13.4 \%$ 
growth-inhibition for the control strain MRC10. The mutant with the highest sensitivity was myco2694, which showed greater than 95\% inhibition. In addition, two mutants myco1030 and myco4003, stood out with greater than $60 \%$ inhibition.

Table 3.4: Growth-inhibition of top secondary assay hits versus rifampicin.

\begin{tabular}{|c|c|}
\hline Strain & Growth-inhibition $^{\mathrm{a}} \pm$ se (\%) \\
\hline myco2694 & $97.7 \pm 1.92$ \\
\hline myco4005 & $70.0 \pm 22.0$ \\
\hline myco1030 & $61.0 \pm 19.1$ \\
\hline myco5231 & $45.0 \pm 17.2$ \\
\hline myco0163 & $39.1 \pm 20.0$ \\
\hline myco7011 & $38.1 \pm 31.9$ \\
\hline myco891 & $38.0 \pm 25.8$ \\
\hline myco5826 & $35.2 \pm 21.6$ \\
\hline myco6762 & $35.1 \pm 33.8$ \\
\hline myco6859 & $33.6 \pm 4.56$ \\
\hline myco2642 & $31.3 \pm 11.3$ \\
\hline$m y c 05883$ & $31.1 \pm 0.73$ \\
\hline MRC10 & $13.4 \pm 0.30$ \\
\hline
\end{tabular}

${ }^{a}$ Growth-inhibition values represent mean values across three independent assays with standard errors (se) presented.

\subsubsection{Quantification of mutant hypersensitivity}

The rifampicin MIC was determined by dose-response assay (section 2.8.3) for the 12 most sensitive mutants from the secondary assay in order to quantify mutant sensitivity. Rifampicin MICs for the 12 strains were compared to an MIC value averaged across the 10 random transposon mutant control strains (section 3.3.3). In general, the mutant strains showed an increased sensitivity to rifampicin compared to the control strain with 11 of the 12 strains showing a decrease in MIC compared to the control MIC (Table 3.5). The two most sensitive mutants from the secondary assay had the greatest decrease in rifampicin MIC, whereby myco2694 showed an almost eight-fold decrease in MIC, while mutant myco4005 showed a four-fold decrease in MIC. The remaining mutants showed a two-fold or less decrease in MIC to rifampicin compared to the control strain. 
Table 3.5: Sensitivity of transposon mutants to rifampicin.

\begin{tabular}{cc}
\hline Strain & MIC \pm se $(\mu \mathrm{M})$ \\
\hline myco2694 & $0.09 \pm 0.03$ \\
myco4005 & $0.16 \pm 0.00$ \\
myco5826 & $0.26 \pm 0.05$ \\
myco6762 & $0.26 \pm 0.05$ \\
myco1030 & $0.31 \pm 0.00$ \\
myco0163 & $0.31 \pm 0.00$ \\
myco7011 & $0.31 \pm 0.00$ \\
myco5231 & $0.52 \pm 0.10$ \\
myco891 & $0.52 \pm 0.10$ \\
myco2642 & $0.52 \pm 0.10$ \\
myco5883 & $0.52 \pm 0.10$ \\
myco6859 & $0.63 \pm 0.00$ \\
Tn Control & $0.64 \pm 0.04$ \\
\hline
\end{tabular}

Rifampicin MICs for the 12 mutants most sensitive to rifampicin identified in the secondary assay, averaged over three independent experiments. The transposon $(\mathrm{Tn})$ control value represents the mean of the 10 randomly selected mutant control strains. Standard errors (se) are presented.

\subsubsection{Growth curve of rifampicin hypersensitive mutant myco4005}

Interestingly, it was noticed that the rifampicin hypersensitive strain myco4005 (with a four-fold reduction in MIC) took longer to grow than all other rifampicin hypersensitive mutant strains. A growth curve experiment (section 2.9) was performed to measure the reduced growth rate. A control strain (random transposon mutant strain D11), and the rifampicin hypersensitive mutant myco2694 reached stationary phase by 24 hours, whereas the strain myco4005 took approximately 24 hours longer (48 hours total) to reach stationary phase (Figure 3.8). 


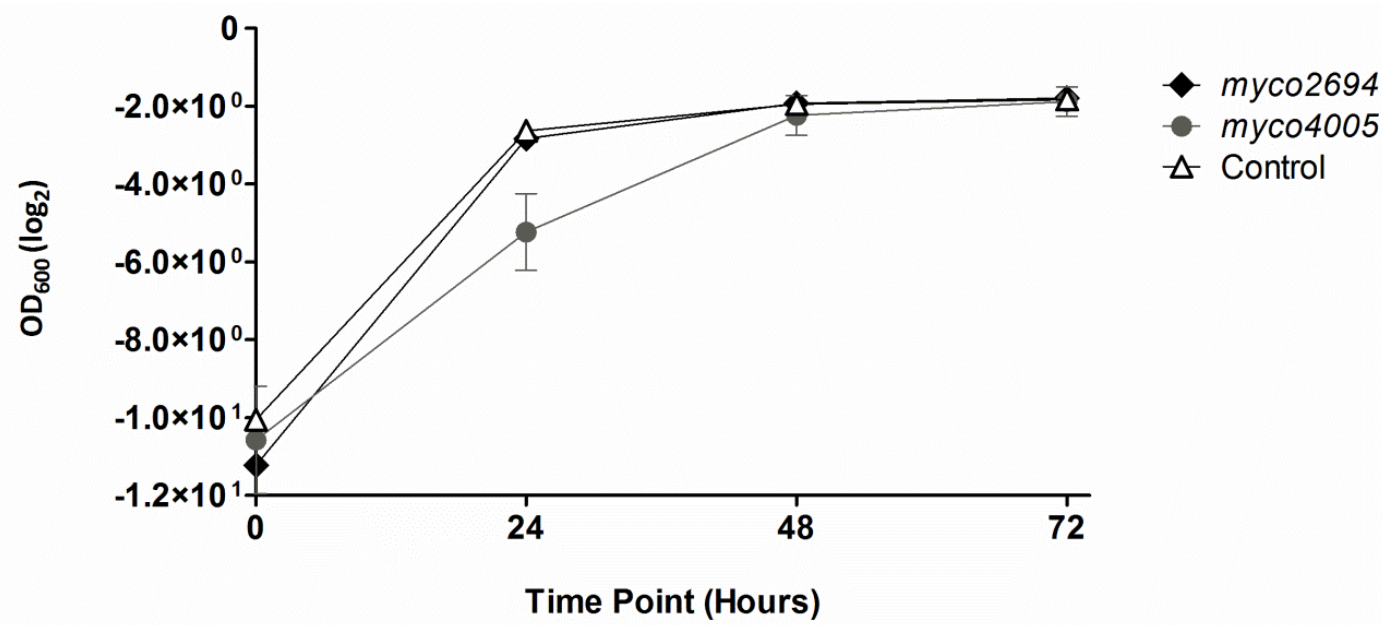

Figure 3.8: Growth curve of rifampicin hypersensitive strains. Growth of the rifampicin hypersensitive strains myco2694 and myco4005, and the random transposon mutant control strain D11, was measured every 24 hours by $\mathrm{OD}_{600}$ up to 72 hours. Values shown are $\log _{2}$ transformed mean $\mathrm{OD}_{600}$ values across three independent experiments with standard error bars.

\subsubsection{Bedaquiline}

\subsubsection{Primary assay for drug hypersensitivity}

The primary assay for bedaquiline hypersensitivity was performed at a concentration of $10.8 \mathrm{nM}$ (Table 3.1). As the sRFU values for the treated-MRC10 controls within each library were normally distributed one-way ANOVA were performed on untransformed data. The one-way ANOVAs did not show a significant difference in mean sRFU for the treated-MRC10 control across all plates or either library A or B $(p>0.05)$, therefore analysis of the assay data continued as described in section 3.2.3.

The median inhibition of mutant library A versus bedaquiline was approximately $49 \%$ (Figure 3.9). The 60 most sensitive mutants from library $A$, representing mutants that were at least 1.76 times more sensitive to bedaquiline than the library median, were picked into the bedaquiline mini-hit library. 
A

B

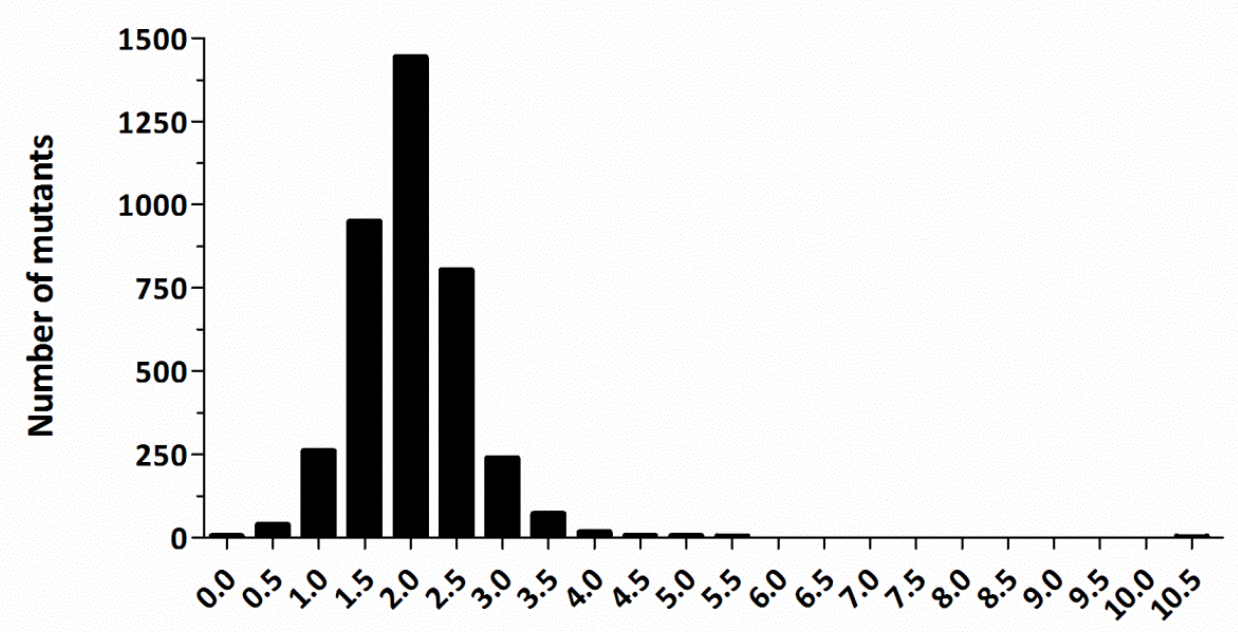

$F_{1}$

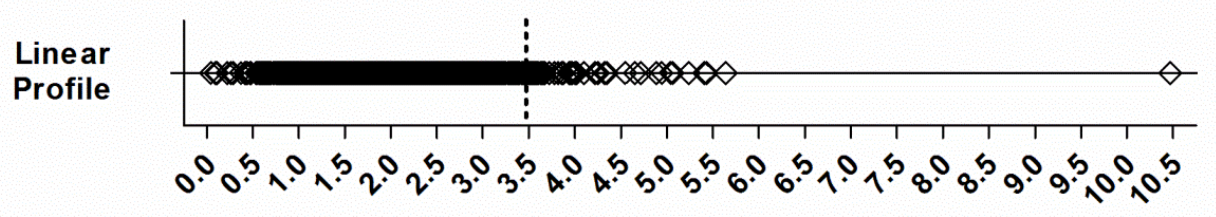

$F_{1}$

Figure 3.9: Mutant library A versus $10.8 \mathrm{nM}$ bedaquiline. (A) Frequency distribution of $F_{1}$ values, and (B) $F_{1}$ linear profile, from library A versus $10 \mathrm{nM}$ bedaquiline. The median $F_{1}$ across mutant library A was 1.97 , and the median $F_{1}$ for the treated-MRC10 control was 2.37 . The dotted line indicates the cut-off for the 60 most sensitive mutants, and lies at $F_{1}=3.47$. The $F_{1}$ value for each mutant represents the mean $F_{l}$ across three independent experiments.

The median inhibition of mutant library B versus bedaquiline was approximately $37 \%$ (Figure 3.10). The 60 most sensitive mutants from library $B$, representing mutants that were at least 1.6 times more sensitive to bedaquiline than the library median, were picked into the bedaquiline mini-hit library. 
A

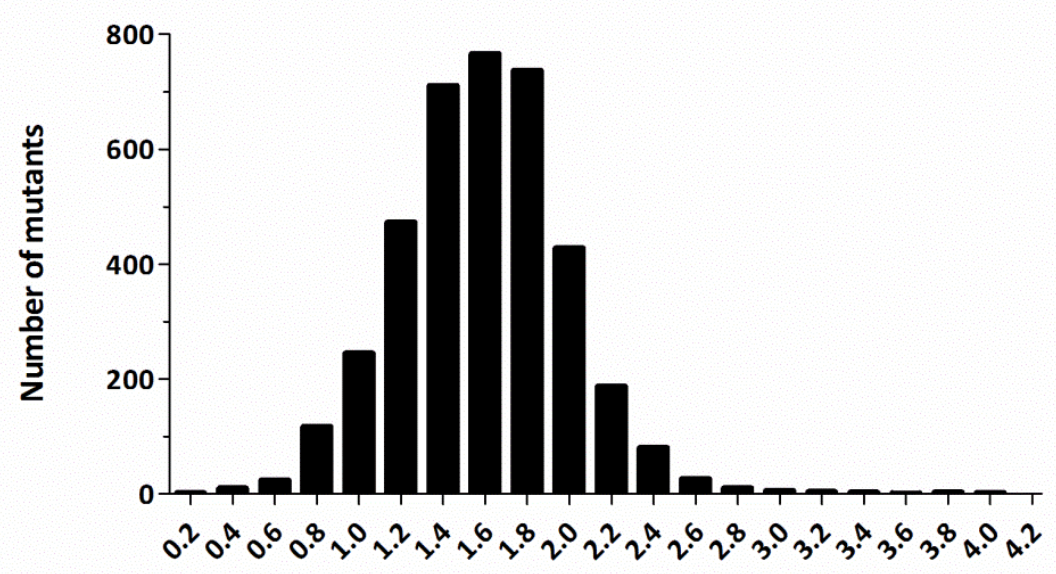

B

$F_{1}$

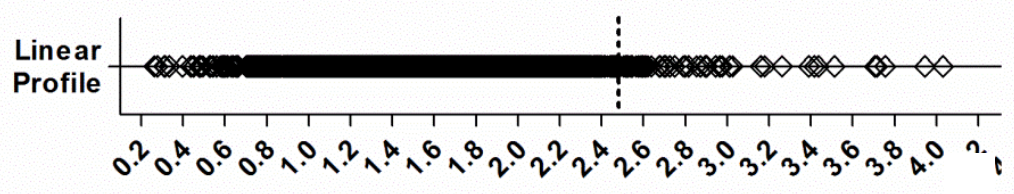

$F_{1}$

Figure 3.10: Mutant library $B$ versus $10.8 \mathrm{nM}$ bedaquiline. (A) Frequency distribution of $F_{1}$ values, and (B) $F_{1}$ linear profile, from library $B$ versus $10.8 \mathrm{nM}$ bedaquiline. The median $F_{1}$ across mutant library $B$ was 1.58 , and the median $F_{1}$ for the treated-MRC10 control was 1.90 . The dotted line indicates the cut-off for the 60 most sensitive mutants, and lies at $F_{1}=2.53$. The $F_{1}$ value for each mutant represents the mean $F_{1}$ across three independent experiments.

\subsubsection{Secondary assay for drug hypersensitivity}

A secondary assay was performed In order to validate the hypersensitivity to bedaquiline of the 120 most sensitive mutants (60 from each library) identified in the primary screen. Each hypersensitive mutant was tested against the screening concentration of bedaquiline (Table 3.1) and growth-inhibition was determined by endpoint $\mathrm{OD}_{600}$ measurement as described in section 3.2.4. The concentration of bedaquiline used in the secondary assay was the same as that used in the primary assay $(10.8 \mathrm{nM})$. Table 3.6 shows the 12 most sensitive mutants from the secondary assay against the bedaquiline mini-hit library. Data for the mutant myco2908 is included as sequencing by collaborators at the University of Otago revealed that it had a transposon insertion in $\operatorname{atp} B$. This gene encodes the A subunit of the ATPase $F_{0}$ domain, linking it to the target of bedaquiline, the $C$ subunit of the ATPase $F_{0}$ domain. 
Mutant myco2908 was the $18^{\text {th }}$ most sensitive mutant identified in the secondary assay for bedaquiline. The mutants in the secondary assay against the bedaquiline mini-hit library showed a median inhibition of $39.3 \%$ compared to only $15.5 \%$ inhibition for the control MRC10. Three mutants, myco3011, myco5040, and myco6564, stood out, showing greater than $80 \%$ inhibition, while the remaining 10 mutants all showed over $50 \%$ inhibition.

Table 3.6: Growth-inhibition percentage of top secondary assay hits versus bedaquiline.

\begin{tabular}{cc}
\hline Strain & Growth-inhibition ${ }^{\mathrm{a}} \pm$ se (\%) \\
\hline myco3011 & $83.8 \pm 2.94$ \\
myco5040 & $83.5 \pm 5.68$ \\
myco6564 & $82.7 \pm 0.96$ \\
myco2833 & $77.2 \pm 1.67$ \\
myco4393 & $73.4 \pm 3.98$ \\
myco1994 & $72.5 \pm 6.19$ \\
myco3491 & $70.5 \pm 9.21$ \\
myco2664 & $70.5 \pm 5.07$ \\
myco1917 & $69.5 \pm 0.52$ \\
myco2176 & $68.4 \pm 5.41$ \\
myco0934 & $61.5 \pm 6.75$ \\
$m y c 06539$ & $61.1 \pm 8.37$ \\
myco2908 & $54.3 \pm 8.65$ \\
MRC10 & $15.5 \pm 2.40$
\end{tabular}

aGrowth-inhibition values represent mean values across three independent assays with standard error (se) presented.

\subsubsection{Quantification of mutant hypersensitivity}

The bedaquiline MIC was determined by dose-response assay (section 2.8.3) for the 12 most sensitive mutants from the secondary assay in order to quantify mutant sensitivity. Bedaquiline MICs for the 12 strains were compared to an MIC value averaged across the 10 random transposon mutant control strains (section 3.3.3). Of the 12 mutants most sensitive to bedaquiline from the secondary assay, only nine showed some level of increased sensitivity when quantified according to their MIC (Table 3.7). Two strains showed a two-fold or greater decrease in MIC compared to the control strain, myco1994 and myco2908. Interestingly, three strains, myco934, myco4393, and myco6539 had higher MIC values than the control mutant average (Table 3.7). 
Table 3.7: Sensitivity of transposon mutants to bedaquiline.

\begin{tabular}{cc}
\hline Strain & MIC \pm se (nM) \\
\hline myco1994 & $18.6 \pm 3.75$ \\
myco2908 & $22.5 \pm 0.00$ \\
myco2833 & $26.3 \pm 9.92$ \\
myco2664 & $33.8 \pm 11.3$ \\
myco3011 & $33.8 \pm 11.3$ \\
myco3491 & $37.5 \pm 7.50$ \\
myco5040 & $37.5 \pm 7.50$ \\
myco6564 & $37.5 \pm 7.50$ \\
myco1917 & $37.5 \pm 7.50$ \\
myco2176 & $37.5 \pm 7.50$ \\
Tn Control & $45.8 \pm 3.25$ \\
Myco0934 & $60.0 \pm 15.0$ \\
myco4393 & $60.0 \pm 15.0$ \\
myco6539 & $75.0 \pm 15.0$ \\
\hline
\end{tabular}

Bedaquiline MICs for the 12 mutants most sensitive to bedaquiline identified in the secondary assay, and myco2908, averaged over three independent experiments. The transposon ( $\mathrm{Tn}$ ) control value represents the mean of the 10 randomly-selected mutant control strains. Standard errors (se) are presented.

\subsection{Discussion}

The aim of this chapter was to identify mutants of $M$. smegmatis $\mathrm{mc}^{2} 155$ that were hypersensitive to isoniazid, rifampicin, and bedaquiline. To this end, a library of random Tn611 transposon mutants was constructed and screened for drug hypersensitivity. Mutants that were hypersensitive were identified for all three drugs and the sensitivities quantified by MIC for the 12 mutants most sensitive in each secondary assay.

\subsubsection{Screening for drug hypersensitivity}

The assay undertaken in this thesis to identify drug hypersensitive mutants in a Tn611 transposon mutant collection was successful, with mutants identified that were sensitive to all of the drugs tested (isoniazid, rifampicin, and bedaquiline). The assay was run at a concentration of drug that caused minor growth-inhibition across the mutant libraries, allowing those mutants that were hypersensitive to the drug to be differentiated from the rest of the transposon mutants. The original target was to use a drug concentration that 
caused $15-30 \%$ median growth-inhibition across a library, however after performing the assays it became apparent that greater variation in the compound's screening concentration can still successfully identify hypersensitive mutants. Specifically, the lowest average inhibition was $5 \%$ in the rifampicin versus library B assay; and the highest was $49 \%$ in the bedaquiline versus library $A$ assay. These results indicate the assay for drug hypersensitivity developed in this thesis was more sensitive than initially suspected. This conclusion is also supported by the increased number of rifampicin hypersensitive mutants identified in this assay compared to a previous one performed by Alexander et al. (2003). In the previous study only one mutant was isolated that showed increased rifampicin sensitivity, and is likely a combined effect of the different type of assay and drug concentration used. Other factors such as library coverage may have also had an impact; both the collection of mutants used in this assay, and that by Alexander et al. (2003) contained 7680 mutants, therefore there would likely be a large, but not complete overlap between the two different libraries. The assay by Alexander et al. (2003) used an endpoint metric that was the presence or absence of growth on solid media containing antibiotic compared to the assay setup used in this study, where a strain's growth does not need to be completely inhibited to be detected. This demonstrates the general higher sensitivity achieved with a qualitative endpoint growthinhibition assay compared to a quantitative growth versus no growth metric.

The assay for drug hypersensitivity was designed for high-throughput with the reduction of resazurin used as the endpoint measure of growth-inhibition. As discussed in the introduction resazurin reduction provides a relative measure of growth-inhibition, with other factors including incubation time with the dye affecting the absolute fluorescence achieved by a culture. The library plates were designed to contain an in-plate control strain, MRC10, to enable standardisation whereby the fluorescence of untreated MRC10 was used as the maximum fluorescence standard to which all other strains on the plate were compared. The reliability of this control was confirmed when the libraries were grown in LB alone and the median standardised fluorescence of the treated-MRC10 showed no significant difference across all plates within a library. The treated-MRC10 controls were also compared for the assays against isoniazid, rifampicin, and bedaquiline, and showed no significant differences within either library for all drugs. This result indicates that the treatment conditions were the same for all plates within a library, and that it was valid to 
directly compare the sensitivity of the mutants between separate plates within a library. As the libraries were screened separately the data analysis was also performed separately to avoid any library bias due to different treatment conditions between libraries $A$ and $B$. It was noted that the treatment conditions were significantly different between the assays against Library A and Library B for rifampicin and bedaquiline, therefore a library bias would have existed had the data been analysed together.

As discussed in the chapter introduction, the use of resazurin, while useful in improving assay throughput, also added a risk of introducing false positives. To overcome this, a secondary assay that used endpoint $\mathrm{OD}_{600}$ was used to validate the sensitivity of the 60 most sensitive mutants from each library for each drug. It was expected that this number of mutants would contain most if not all the true positives, while being small enough to screen in the lower throughput of $\mathrm{OD}_{600}$ based measurement of growth. To this end, the median growth-inhibition of the mini-hit libraries for each of the three drugs was compared to the MRC10 control. It was observed that the median inhibition of the isoniazid and rifampicin mini-hit libraries by the respective drugs was approximately equal to the inhibition of MRC10. This suggests that the majority of mutants within these mini-hit libraries were not sensitive to the drugs and were false positives. Interestingly, median inhibition of the bedaquiline mini-hit library was approximately twice that of the MRC10 strain, suggesting that most of the mutants in the bedaquiline mini-hit library were true positives and hypersensitive to bedaquiline. This high true positive rate may be due to the mode of action of bedaquiline, which inhibits the ATPase enzyme, with mutations in many of the pathways that utilise ATP potentially sensitising the bacteria to bedaquiline (Andries et al., 2005; Koul et al., 2007). These results confirm the original assumption that the top 60 mutants from each library would contain most if not all of the true hits.

It was observed that the growth-inhibition values generated from the secondary assay had relatively large errors associated with them, likely due to the way in which the assay was setup. In order to sub-inoculate the 96 -well plates for the secondary assay, a 96 well pinning tool was used in order to replicate the manner in which the primary assay was setup. This pinning resulted in the occasional transfer of visible clumps of cells, and this variation in starting cell number likely affected the final growth measurement. Two biological replicates were performed for each assay replicate, however increasing this to three of four biological 
replicates would likely have reduced the errors; additionally, sub-inoculation of the assay plates by pipetting would have likely decreased variation in the assay. Despite these larger errors the secondary assay was successfully used to validate the sensitivity of the mutants identified in the primary assay.

\subsubsection{Quantifying drug sensitivity}

MICs were determined for the top $10 \%$ of sensitive mutants (12 strains) from the secondary assays for each of the drugs tested, and were used as a convenient metric for quantifying drug sensitivity. For bedaquiline an additional mutant, myco2908, was included as it contained a transposon insertion within $a t p B$, a gene associated with the known mode of action of bedaquiline, as identified by collaborators at the University of Otago. The control for this assay was an averaged MIC across 10 random mutant strains from the transposon libraries. The random mutant strains were included to account for differences in basal sensitivity between a transposon mutant and the non-transposon mutant control MRC10 as a result of the transposon insertion. These 10 mutant control strains were not identified as hypersensitive to isoniazid, rifampicin, or bedaquiline in either the primary or secondary assays. However, to eliminate the likelihood of accidently choosing a single random transposon mutant strain that had an altered sensitivity to one of the drugs, an average across the 10 strains was used. It was found that there was no significant difference in the MIC values between the control strains for isoniazid, rifampicin, or bedaquiline.

Most of the top $10 \%$ of drug hypersensitive mutants from the secondary assay examined showed a two-fold or less decrease in MIC compared to the control average. Several mutants with a greater than two-fold difference in MIC were identified for each respective drug: three for isoniazid, two for rifampicin, and two for bedaquiline. These were fewer mutants than expected, possibly representing a limitation of using MIC to quantify the sensitivity of these mutants. Additionally, the transposon control average MIC to bedaquiline was found to be lower than that of several validated bedaquiline sensitive mutants. This would indicate that the 10 random mutant control strains may also be hypersensitive to bedaquiline, which would agree with the high true positive rate observed for the secondary 
assay for bedaquiline. Therefore, these random transposon strains may not have been the most reliable control for the assay determining MIC values for bedaquiline.

\subsubsection{Summary}

A transposon mutant collection was constructed in a 384-well format and was screened in high-throughput for mutants that were hypersensitive to isoniazid, rifampicin, and bedaquiline. For each drug, 12 validated hypersensitive mutants had their sensitivities quantified by determining their MICs for the relevant drug, with three mutants from the isoniazid assay, two from the rifampicin assay, and one mutant from the bedaquiline assay, standing out. The genotypic basis for drug hypersensitivity for these 36 validated mutants will be further examined in Chapter Four. 


\section{Genotypic validation of drug hypersensitive mutants of M. smegmatis}

\subsection{Introduction}

As discussed in Chapter Three, the advantage of transposon mutagenesis is that the site of the genetic mutation responsible for the drug hypersensitivity is labelled by the transposon sequence. Rapid identification of the transposon insertion site facilitates downstream genetic analysis including complementation of the transposon mutant with an intact plasmid-borne copy of the gene, or overexpression of the identified gene in a WT strain. Complementation determines whether the identified gene is responsible for the drug hypersensitivity phenotype. In mutants where loss of function of the mutated gene causes drug hypersensitivity, complementation of this gene will likely restore the drug sensitivity to levels similar to that seen in WT. Over-expression of these genes of interest in WT allows further characterisation of their role in drug sensitivity, such as whether overexpression results in sensitivity similar to WT or a dramatic increase in drug resistance. Complementation and over-expression experiments, along with information gleaned from the literature, can be combined to generate a more encompassing description of drug mode of action.

\subsubsection{Transposition site identification}

A major advantage of the use of transposons for random mutagenesis is that the site of insertion is labelled with a known DNA sequence (i.e. the transposon). The first step in identifying the site of mutation is to digest the mutant gDNA with a restriction enzyme to yield DNA fragments that contain the junction between the end of the transposon and the flanking gDNA sequence. Multiple techniques exist for amplifying this transposon DNA/gDNA junction; for example, inverse PCR involves ligating the hybrid transposon DNA/gDNA fragments under dilute conditions to produce circularised DNA. The flanking gDNA region can then be amplified from two primers based within the transposon DNA sequence (Billman-Jacobe et al., 2006). An alternative method is ligation-mediated PCR, where a linker is ligated to the transposon DNA/gDNA junction containing fragments and this construct is amplified using a primer from the linker and a primer from the transposon sequence 
(Prod'hom et al., 1998). Sequencing of the PCR products from either inverse PCR or ligation-mediated PCR allows pinpointing of the junction between transposon and gDNA and therefore the specific transposon insertion site.

\subsubsection{Conditional gene expression in mycobacteria}

A number of different constitutive and conditional promoter systems have been developed to allow the extra-chromosomal or integrated expression of genes in mycobacteria (Blokpoel et al., 2005; Brown and Parish, 2006; Carroll et al., 2005, 2007; Ehrt et al., 2005; Pandey et al., 2009; Stover et al., 1991; Triccas et al., 1998). Conditional promoters come in a number of varieties including on/off type promoters and titrateable promoters, both of which can be actively induced and/or repressed by small molecules depending on the specific promoter. Tetracycline inducible promoters are routinely used in mycobacteria for conditional gene expression, and are activated when tetracycline binds to a repressor protein, allowing the RNA polymerase access to the promoter and resulting in induction of gene expression (Figure 4.1). The common limitation of the conditional expression vectors, including the tetracycline inducible vectors, is that the promoter can be leaky, with potentially high levels of basal expression in the absence of the inducer tetracycline. Newer generation vectors, such as plasmid pKW08 (Williams et al., 2010), have been constructed to limit basal expression in mycobacteria, although the vector is still leaky in $E$. coli, which is routinely used for sub-cloning. A derivative of plasmid pKW08 will be used for the conditional gene expression in $M$. smegmatis $\mathrm{mc}^{2} 155$ in this thesis. 
A

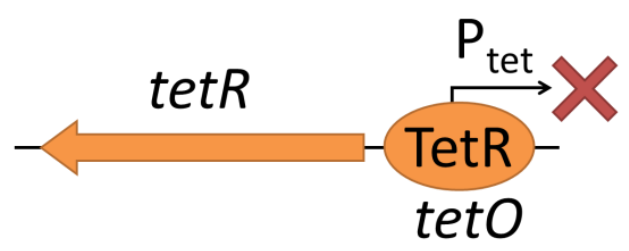

B

TetR

- Tetracycline

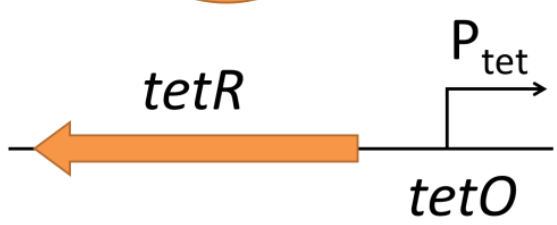

Figure 4.1: Tetracycline inducible promoter of plasmid pKW08. (A) The repressor protein TetR, encoded by tet $R$, binds the tetO operator region and prevents transcription from the promoter $\mathrm{P}_{\text {tet }}$. (B) The inducer (tetracycline) binds the repressor protein (TetR) and prevents binding of TetR to tetO, allowing transcription from $P_{\text {tet }}$.

\subsubsection{Objectives of Chapter Four}

The goal of Chapter Four was to identify the genetic basis for the drug hypersensitivity phenotype of the 12 most sensitive transposon mutants validated in each secondary assay (isoniazid, rifampicin, and bedaquiline assays for drug hypersensitivity) in Chapter Three.

To achieve this goal the following specific aims were addressed:

- Identification of the transposon insertion site in the 12 mutants most hypersensitive to isoniazid, rifampicin, and bedaquiline from Chapter Three

- Complementation of a select few transposon mutants to confirm the role of the gene containing the transposon insertion in these strains drug sensitivity

- Overexpression of genes containing or adjacent to Tn611 insertions in drug hypersensitive mutants to further characterise their role in drug sensitivity

The site of Tn611 insertion in the isoniazid, rifampicin, and bedaquiline hypersensitive mutants of $M$. smegmatis $\mathrm{mc}^{2} 155$ was determined using either inverse PCR or ligation-mediated PCR. Genes identified as containing, or adjacent to Tn611 insertions were 
expressed from the tetracycline inducible promoter of pRC20, a derivative of plasmid pKW08, in Tn611 mutants for complementation assays, or in WT M. smegmatis mc $^{2} 155$ for overexpression assays.

\subsection{Methods}

\subsubsection{Transposon insertion site identification}

Two different methods were used to identify the insertion site of the Tn611 in the mutant strains of $M$. smegmatis $\mathrm{mc}^{2} 155$ : inverse PCR or ligation-mediated PCR. Inverse PCR was performed initially, however was not successful for generating sequences for all of the mutants tested. In such cases, ligation-mediated PCR was subsequently performed to enable identification of the transposon insertion site.

\subsubsection{Inverse PCR}

Substrates for inverse PCR were generated as described in section 2.6.4, and inverse PCR performed as described in section 2.6.3.2. The products from inverse PCR were electrophoresed on a $1 \%$ agarose gel (section 2.6.1.4). Inverse PCR produced two products: one of constant size (approximately $1 \mathrm{~Kb}$ ) that corresponded to an internal transposon PCR product; and a variable sized band that correlated to the product containing the gDNA/junction. Gel extraction was performed to isolate the variably sized band of interest as described in section 2.6.1.5. The gDNA flanking the Tn611 insertion was subsequently sequenced using primer 3054 ( Table 2.4 . 
Table 2.4). If a PCR product could not be generated by inverse PCR from the Rsal generated fragments, or the PCR product did not provide a useful sequence, the inverse PCR process was repeated using another restriction enzyme, Eagl. The protocol for Eagl based inverse PCR was identical to Rsal based inverse PCR except that the DNA fragments were generated by Eagl digestion instead (section 2.6.4.)

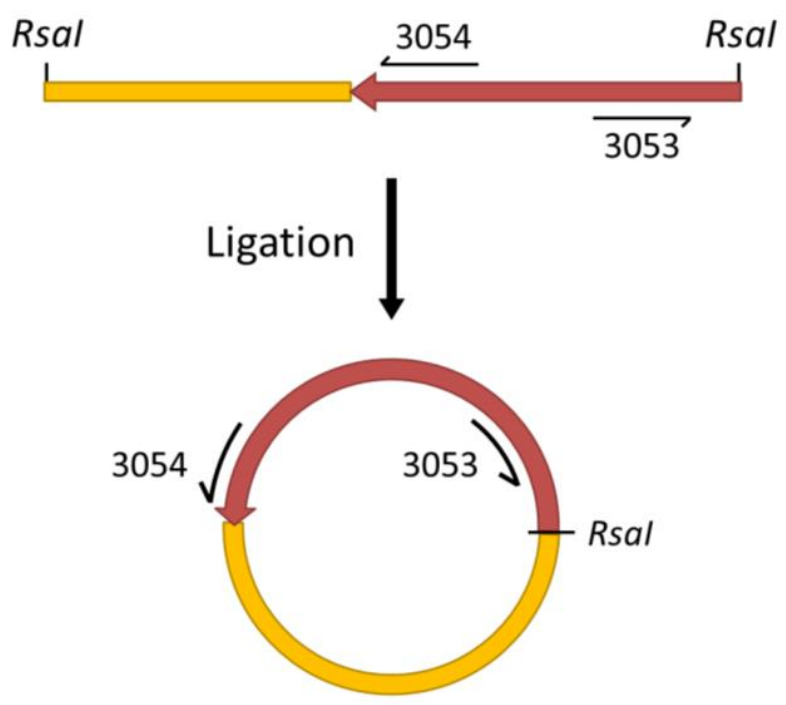

Figure 4.2: Inverse PCR. Rsal digested gDNA from a Tn611 insertional mutant is circularised by ligating under dilute conditions. The outward facing transposon primers 3053 and 3054 are then able to amplify the gDNA/transposon DNA junction. Red bars represent Tn611 DNA, while yellow bars represent $M$. smegmatis $\mathrm{mc}^{2} 155$ gDNA.

\subsubsection{Ligation-mediated PCR}

In cases where inverse PCR with either Rsal or Eagl digestion failed to produce a PCR product or produced a PCR product that failed to give a useful sequence, ligation-mediated PCR was used instead. ligation-mediated PCR was performed as described previously by Prod'hom et al., (1998) and as depicted in Figure 4.3. The substrate for ligation-mediated PCR was generated as described in section 2.6.5, and ligation-mediated PCR performed as described in section 2.6.3.2. PCR products were electrophoresed on $1 \%$ agarose gels (section 2.6.1.4) and successful PCR reactions were cleaned and concentrated (section 2.6.1.6) and sequenced with salgd and OligoF (Table 2.4). 


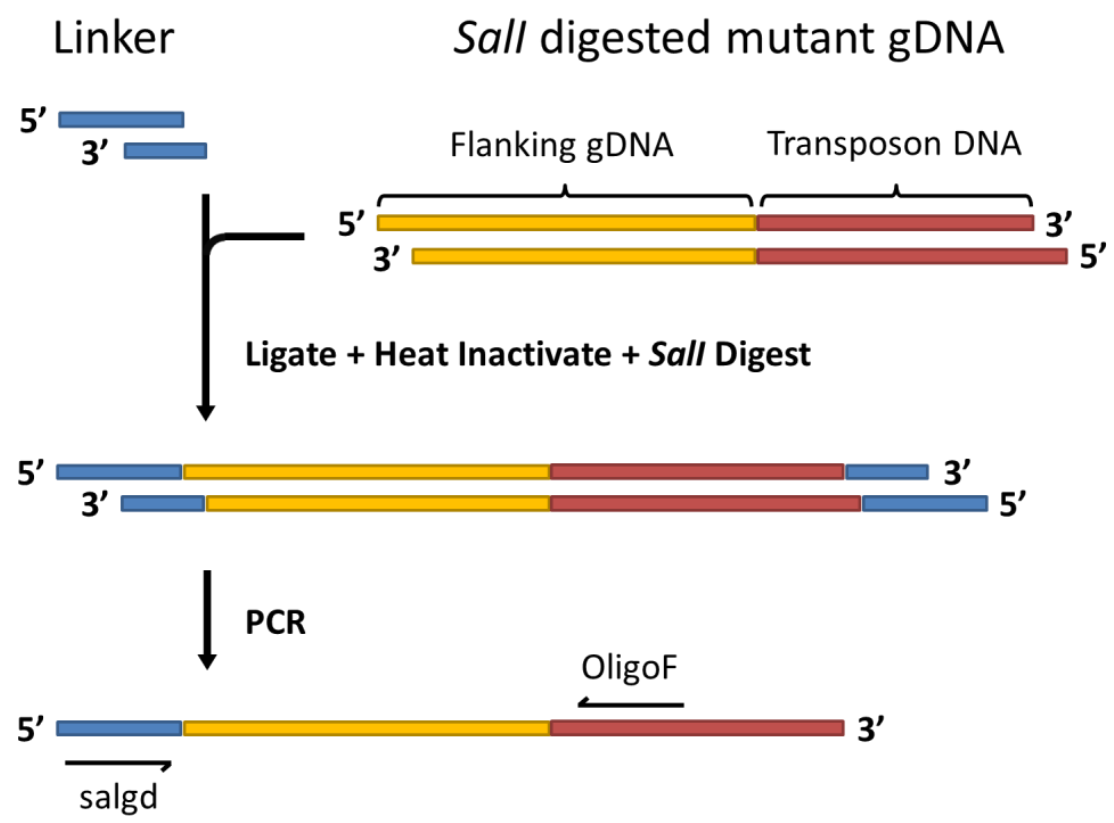

Figure 4.3: Ligation-mediated PCR. The linker (blue), composed of the non-phosphorylated oligos salgd and salpt, is ligated to Sall digested transposon mutant DNA fragments. While the longer salgd oligo strand can ligate to the DNA fragment due to the fragments 5 ' phosphate group, the shorter salpt strand lacks its own phosphate so cannot ligate and subsequent heating during PCR results in the salpt oligo being displaced. The second digestion with Sall ensures no unwanted products are amplified during PCR due to improper initial Sall digestion. The fragment containing the gDNA flanking the transposon mutant is selectively amplified by PCR using the linker primer salgd, and OligoF.

\subsubsection{Sequence processing}

The PCR products generated by inverse PCR or ligation-mediated PCR as described previously, were sequenced as described in section 2.6.2. Sequences of the PCR products generated from inverse PCR or ligation-mediated PCR were analysed identically using the bioinformatics software Geneious 5.6.6 (www.geneious.com) as follows. The returned DNA sequence was aligned with the DNA sequence from the end of the Tn611 transposon using the Geneious "pairwise align" function with default settings to identify the junction between the transposon and gDNA. The sequence of the gDNA from the transposon DNA/gDNA junction until the first relevant restriction site (Rsal or Eagl or Sall), or until the end of the 
sequence (whichever was shorter), was used as the input for a nucleotide Basic Local Alignment Search Tool (BLAST) analysis at the ncbi BLAST website (http://blast.ncbi.nlm.nih.gov). The nucleotide BLAST was performed against the nonredundant nucleotide database using default settings to identify the genomic region matching the gDNA sequence generated from inverse PCR/ligation-mediated PCR. Once identified, the corresponding genomic region was used as a reference sequence against which the PCR generated gDNA sequence was aligned in Geneious using the "align to reference" tool with default settings. From this reference alignment the exact location of the Tn611 insertion in the M. smegmatis $\mathrm{mc}^{2} 155$ genome was determined. The identity, function, and known M. tuberculosis homologues of genes containing transposon insertions, or genes adjacent to intergenic transposon insertions, was determined using databases including tuberculist (Lew et al., 2011), smegmalist (Kapopoulou et al., 2011), and the TBDB (Galagan et al., 2010). If no M. tuberculosis homologue was given for a $M$. smegmatis gene on any of these databases then none was reported in the tables of results for each drug later in this chapter.

\subsubsection{Optimisation of tetracycline concentration for induction of gene expression from plasmid pRC20}

The plasmid pRC20 was used for both the complementation and overexpression assays, and the optimal tetracycline inducer concentration was determined for each of these assays separately. Dose-response assays measuring endpoint GFP fluorescence and growth-inhibition with varying concentrations of tetracycline were performed to optimise the tetracycline concentrations (section 2.8.3). For the complementation assays, the complementation strains and empty vector controls were grown in $0,2.5,5,10,20$, or 40 $\mathrm{ng} \cdot \mathrm{mL}^{-1}$ tetracycline, under the same conditions as the complementation assays (specifically $41{ }^{\circ} \mathrm{C}$ and $20 \mu \mathrm{g} \cdot \mathrm{mL}^{-1}$ kanamycin and hygromycin B). For the overexpression assays, the empty vector control strain MRC20 was grown in $0,5,10,20,40$, or $80 \mathrm{ng} \cdot \mathrm{mL}^{-1}$ tetracycline, under the same conditions as the overexpression assays $\left(37^{\circ} \mathrm{C}\right.$ and $50 \mu \mathrm{g} \cdot \mathrm{mL}^{-1}$ hygromycin B). The optimal tetracycline concentration for each assay was determined as the 
concentration that provided maximal GFP fluorescence, and therefore gene expression from the tetracycline inducible promoter of pRC20, while causing minimal growth-inhibition.

\subsubsection{Complementation of transposon mutants}

Complementation was performed using the vector pRC20 expressing the WT copy of the gene containing or adjacent to the Tn611 insertion in the relevant mutant strain. The WT copies of the genes were amplified by PCR from $M$. smegmatis $m^{2} 155$ (section 2.6.3.2) before being cloned into the BamHI site in plasmid pRC2O as described in section 2.6.9.1. Sequencing was performed to check the orientation and fidelity of the insert as described in section 2.6.2. Constructs that were in the correct orientation and did not contain errors were transformed into the relevant transposon mutant strain (section 2.6.7.2). One gene used for complementation, MSMEI_1905, contained a BamHI site within its ORF which was removed by PCR using a long (60-mer) primer to remove the restriction site by creating a silent mutation (Table 2.5). The removal of the restriction site was confirmed by sequencing as described in section 2.6.2.

Empty vector control strains were generated for each transposon mutant strain by transforming the transposon mutant strains with pRC20 (section 2.6.9). Complementation assays were performed as dose-response assays as described in section 2.8.3. The MIC of the relevant drug was determined for the complementation strains and empty vector controls, both with and without vector induction by tetracycline.

\subsubsection{Overexpression of $M$. smegmatis genes}

The vectors expressing the genes of interest generated for the complementation assays were also transformed into WT $M$. smegmatis $\mathrm{mc}^{2} 155$ to generate the corresponding overexpression strains. Overexpression assays were performed as dose-response assays as described in section 2.8.3. The MIC of the relevant drug was determined for the overexpression strains, both with and without vector induction by tetracycline. Control strains for the overexpression assay included an empty vector negative control strain (MRC20), and an additional negative control strain that expressed a random gene from 
M. smegmatis $\mathrm{mc}^{2} 155$. This negative control strain overexpressed MSMEI_4267, the gene in which the random transposon control strain D11 contained a Tn611 insertion. MSMEI_4267 encodes a putative iron-sulfur oxidoreductase. For the overexpression assays against isoniazid an additional positive control expressing the gene encoding the known target of isoniazid, inhA (MSMEI_3070), was included.

\subsection{Results}

\subsubsection{Isoniazid}

\subsubsection{Transposon insertion site identification}

The transposon insertion sites were able to be identified for the 12 mutants most sensitive to isoniazid identified in Chapter Three, using either inverse PCR or ligation-mediated PCR as described in section 4.2.1. (Table 4.1). 
Table 4.1: Transposon insertion sites in isoniazid hypersensitive mutants.

\begin{tabular}{|c|c|c|c|c|c|c|}
\hline $\begin{array}{l}\text { Mutant } \\
\text { Strain }\end{array}$ & Tn611 insertion location (ORF) & Putative Identification & $\begin{array}{l}\text { Common } \\
\text { Name }\end{array}$ & $\begin{array}{l}\text { Nearest } \mathrm{H}_{37} \mathrm{Rv} \\
\text { homologue }\end{array}$ & $\begin{array}{l}\text { Gene } \\
\text { Length (bp) }\end{array}$ & POI \\
\hline myco0409 & MSMEI_3684 & acetylornithine aminotransferase & $\arg D$ & $\operatorname{Rv} 1655$ & 1173 & 152 \\
\hline myco1897 & MSMEI_5538 & Multiple antibiotic resistance regulator & $\operatorname{mar} R$ & $R v 0880$ & 432 & 385 \\
\hline myco2100 & $\begin{array}{c}55 \text { bp downstream of } M S M E I \_1210 \\
96 \text { bp upstream of } M S M E I \_1211\end{array}$ & $\begin{array}{c}\text { MSMEI_1210 - PAPS reductase } \\
\text { MSMEI_1211 - conserved hypothetical protein }\end{array}$ & - & - & $\begin{array}{c}909 \\
1437\end{array}$ & - \\
\hline myco2576 & MSMEI_1904 & NADH pyrophosphatase & nudC & Rv3199c & 936 & 710 \\
\hline myco2659 & MSMEI_0062 & ftsk/spoiiie family protein & $e c c C a_{1}$ & $R v 3870$ & 2229 & 2072 \\
\hline myco3113 & $\begin{array}{l}47 \text { bp upstream } M S M E I \_4423 \\
338 \text { bp upstream of } M S M E I \_4424\end{array}$ & $\begin{array}{c}\text { MSMEI_4423 - conserved hypothetical protein } \\
\text { MSMEI_4424 - Major Membrane Protein } 1\end{array}$ & - & - & $\begin{array}{c}1278 \\
924\end{array}$ & - \\
\hline myco5016 & MSMEI_1905 & Glutaredoxin-like protein & - & $\operatorname{Rv} 3198 A$ & 249 & 59 \\
\hline myco6166 & MSMEI_5554 & Conserved hypothetical protein & - & $R v 0862 c$ & 2256 & 666 \\
\hline myco6192 & MSMEI_5244 & $\mathrm{K}+$ transporting ATPase, $\mathrm{A}$ subunit & $k d p A$ & $R v 1029$ & 1671 & 391 \\
\hline myco6482 & MSMEI_5460 & putative transcriptional regulator & - & - & 951 & 332 \\
\hline myco7102 & $\begin{array}{l}103 \text { bp upstream of } M S M E I \text { 4764 } \\
\text { 36bp downstream of MSMEI_4765 }\end{array}$ & $\begin{array}{c}\text { MSMEI_4764 - integral membrane transporter } \\
\text { MSMEI_4765 - Alkylhydroperoxidase }\end{array}$ & $\stackrel{-}{a h p D}$ & $\begin{array}{c}- \\
R v 2429\end{array}$ & $\begin{array}{c}1323 \\
534\end{array}$ & - \\
\hline myco7437 & MSMEI_6092 & Fur Family Transcriptional Regulator & - & - & 465 & 204 \\
\hline
\end{tabular}

Isoniazid hypersensitive mutants are organised by their location in the mutant collection in ascending order. Transposon point of insertion (POI) is counted from the start of the gene. 
The myco7437 mutant strain contained a transposon insertion in a putative ferric uptake regulator (Fur) type transcriptional regulator (MSMEI_6092). KatG, the enzyme responsible for the activation of isoniazid, is regulated by the Fur type regulator FurA. Due to the relationship between a Fur type regulator and the mode of action of isoniazid, the similarity between MSMEI_6092 and other Fur type regulators (FurA and FurB type) from M. smegmatis and $M$. tuberculosis was investigated. A protein alignment (Geneious "pairwise alignment", default settings) was performed with the two known M. smegmatis $\mathrm{mc}^{2} 155$ homologs of FurA, FurA1 $1_{\text {SMEG }}\left(M S M E I_{\text {_6215) }}\right.$ and FurA2 $2_{\text {SMEG }}$ (MSMEI_3379), as well as FurB (MSMEI_4376), along with FurA and FurB from M. tuberculosis $\mathrm{H}_{37} \mathrm{Rv}$ (Rv1909c and Rv2359 respectively). The protein alignment showed that MSMEI_6092 is much more similar to the FurA proteins than FurB (Table 4.2). However, the two M. smegmatis FurA proteins are much more similar to each other and Rv1909c than to MSMEI_6092.

Table 4.2: Protein similarity matrix of Fur proteins from $M$. smegmatis $\mathbf{m c}^{2} 155$ and $M$. tuberculosis $\mathrm{H}_{37} \mathrm{Rv}$.

\begin{tabular}{|c|c|c|c|c|c|c|}
\hline Protein & Fur $_{\text {TB }}$ & FurA1 $1_{\text {SMEG }}$ & FurA2 ${ }_{\text {SMEG }}$ & $\begin{array}{c}\text { MSMEI_ } \\
6092\end{array}$ & FurB $B_{\text {SMEG }}$ & FurB $_{\text {TB }}$ \\
\hline Fur $A_{T B}$ & - & 68 & 69.1 & 56.4 & 24.3 & 24.6 \\
\hline FurA1 $_{\text {SMEG }}$ & 68 & - & 65.1 & 56.6 & 22.1 & 24.6 \\
\hline FurA2 $2_{\text {SMEG }}$ & 69.1 & 65.1 & - & 53.4 & 22.1 & 21.6 \\
\hline MSMEI_6092 & 56.4 & 56.6 & 53.4 & - & 25.7 & 26.9 \\
\hline FurB & 24.3 & 22.1 & 22.1 & 25.7 & - & 76.7 \\
\hline FurB $_{\text {TB }}$ & 24.6 & 24.6 & 21.6 & 26.9 & 76.7 & - \\
\hline
\end{tabular}

Percentage similarity of amino acid sequences for M. smegmatis $\mathrm{mc}^{2} 155$ and M. tuberculosis $\mathrm{H}_{37} \mathrm{Rv}$ FurA and FurB proteins and the Fur-like protein encoded by MSMEI_6092.

\subsubsection{Complementation of isoniazid hypersensitive mutants}

The three mutants most hypersensitive to isoniazid identified in Chapter Three, myco2576, myco5016, and myco7437, were selected for complementation using the genes that were identified in 4.3.1.1 as containing the Tn611 insertion in these strains. These strains were selected for complementation as they were greater than two-fold more sensitive to isoniazid than the control whereas the remaining nine hypersensitive strains were between 1.1- and 1.7-fold more sensitive (section 3.3.4.3). It was expected that the greater sensitivity of the selected strains would make it easier to determine whether or not complementation was 
successful. The mutant strains myco2576, myc05016, and myco7437 are henceforth referred to by the ORF containing the Tn611 insertion: Tn::nudC, Tn::MSMEI_1905, and Tn::MSMEI_6092 respectively. A complementation construct containing MSMEI_6092 was unable to be produced. Sequencing results showed that expression plasmids with MSMEI_6092 in the forward direction could be recovered, with a number of constructs containing no insert, or deletions of part of the downstream GFP gene (Figure 4.4).

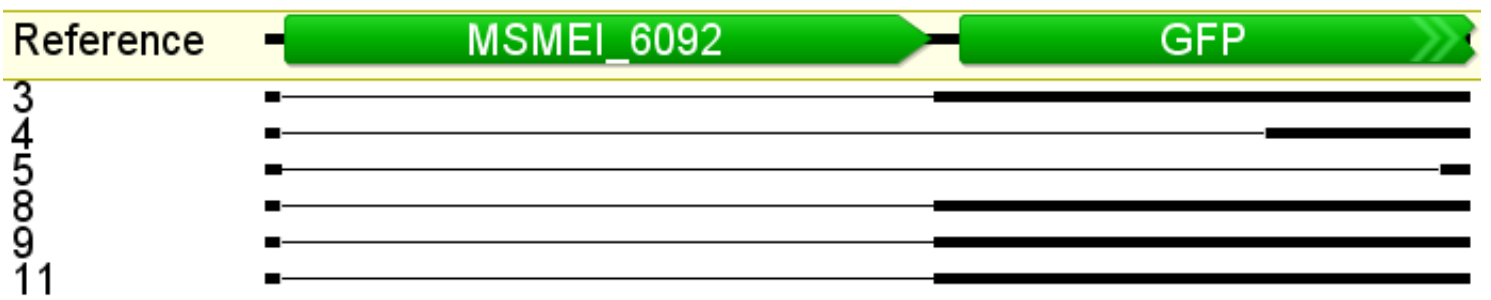

Figure 4.4: Alignments of pRC20 MSMEI_6092 constructs. Sequencing results from the pRC20 constructs containing MSMEI_6092, aligned with a reference sequence. Construct sequences from six recovered clones are shown, with clone number shown on the left hand side. Solid black bars represent sequence that matches the reference, while the thin black lines represent gaps in the sequence. Constructs $3,8,9$, and 11 appear to have no insert, while constructs 4 and 5 contain deletions of part of the GFP gene. All other constructs recovered had MSMEI_6092 in the reverse orientation with no deletions.

The complementation strains for Tn::nudC and Tn::MSMEI_1905 were created by transforming each mutant strain with the pRC20 vector containing either the nudC or MSMEI_1905 construct respectively. Each mutant strain was also transformed with pRC20 that did not contain a construct (i.e. empty vector controls). The inducer concentration was optimised for the complementation studies, with GFP fluorescence used as a relative measure of vector induction, as described in section 4.2.2. As there was no difference between the response of any of the mutant strains or the empty vector strains, these results were analysed together. A tetracycline concentration of $20 \mathrm{ng} \cdot \mathrm{mL}^{-1}$ was found to provide the highest fluorescence, however this concentration showed a substantial increase in growth-inhibition compared to the lower tetracycline concentrations (Figure 4.5). A tetracycline concentration of $10 \mathrm{ng} \cdot \mathrm{mL}^{-1}$ provided an approximate four-fold increase in 
fluorescence, with minimal growth-inhibition, when averaging the response of the four strains, and was therefore selected as the inducer concentration to be used for the complementation assays.

A

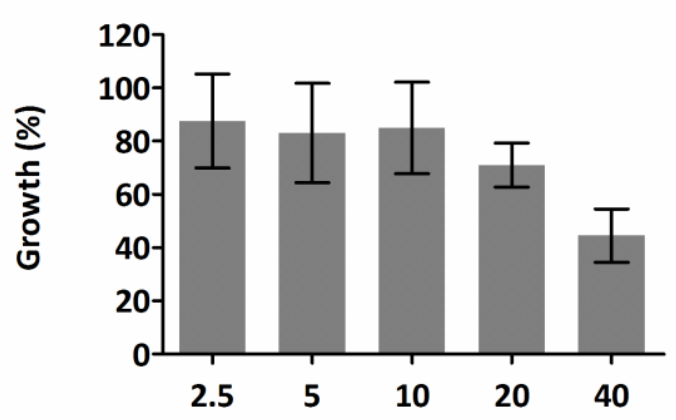

$[\mathrm{Tc}]\left(\mathrm{ng} \cdot \mathrm{mL}^{-1}\right)$
B

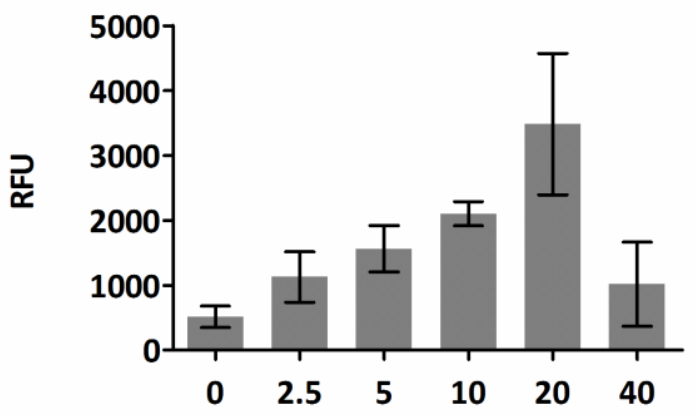

$[\mathrm{Tc}]\left(\mathrm{ng} \cdot \mathrm{mL}^{-1}\right)$

Figure 4.5: Optimisation of inducer concentration for complementation assays. (A) Average endpoint growth relative to the untreated control, and (B) average endpoint RFU of Tn::nudC and Tn::MSMEI_1905 empty vector control and complemented strains grown in different tetracycline (Tc) concentrations. Column heights represent mean values from three independent experiments with standard error bars shown.

The MIC of isoniazid for the relevant complementation strains and the empty vector controls was determined by a dose-response assay (Figure 4.6). There was no change in the MIC of isoniazid for either of the empty vector controls upon induction with tetracycline. The complemented $T n:: n u d C$ strain showed an increased resistance to isoniazid upon induction, with a greater than 10 -fold increase in the MIC, while there was no change in the MIC of isoniazid for the complemented Tn::MSMEI_1905 strain upon vector induction. 


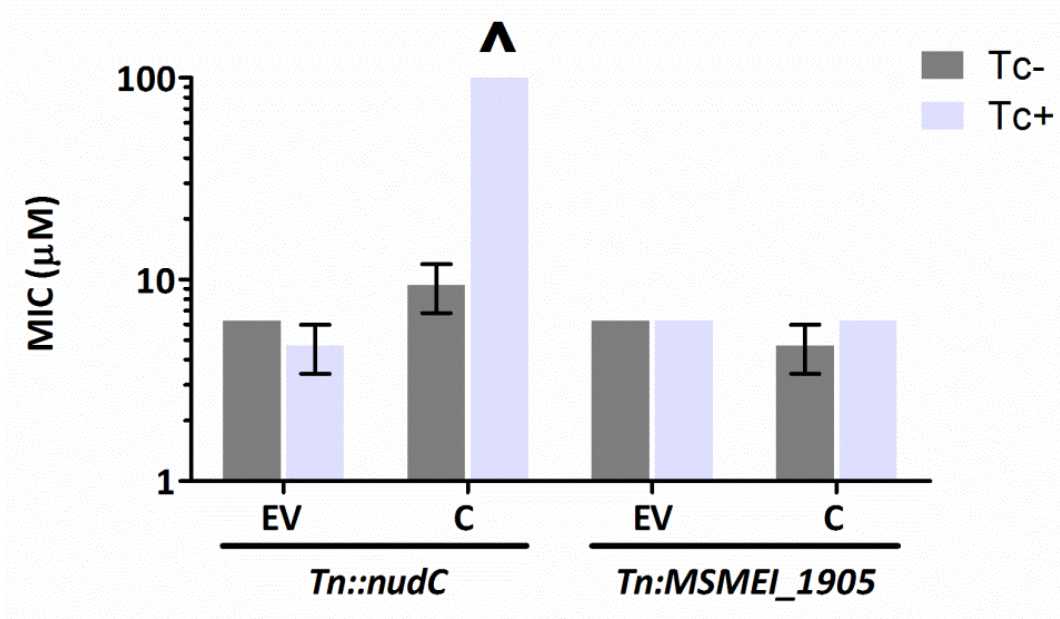

Strain

Figure 4.6: Complementation of isoniazid hypersensitive transposon mutants. The MIC of isoniazid for the Tn::nudC and Tn::MSMEI_1905 empty vector controls (EV) and complemented strains (C) was determined with and without vector induction with $10 \mathrm{ng} \cdot \mathrm{mL}^{-1}$ tetracycline (Tc). Column heights represent mean MIC over three independent experiments, with standard error bars shown. For those columns that have no error bars the standard error was zero. The highest tested concentration of isoniazid was $100 \mu \mathrm{M}$ and MICs greater than the highest tested concentration are indicated by a caret (^).

\subsubsection{Overexpression of genes related to isoniazid hypersensitivity}

The inducer concentration for the overexpression assay was optimised by growing the empty vector control strain (MRC20) in different concentrations of the inducer tetracycline. It was found that a tetracycline concentration of $20 \mathrm{ng} \cdot \mathrm{mL}^{-1}$ gave the highest increase in fluorescence (six-fold) with minimum growth-inhibition. Therefore, $20 \mathrm{ng} \cdot \mathrm{mL}^{-1}$ was selected as the tetracycline concentration for the overexpression assays (Figure 4.7). 
A

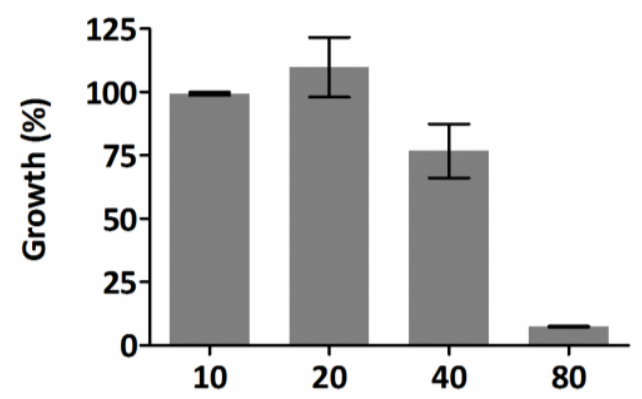

[Tc] (ng $\left.\cdot \mathrm{mL}^{-1}\right)$
B

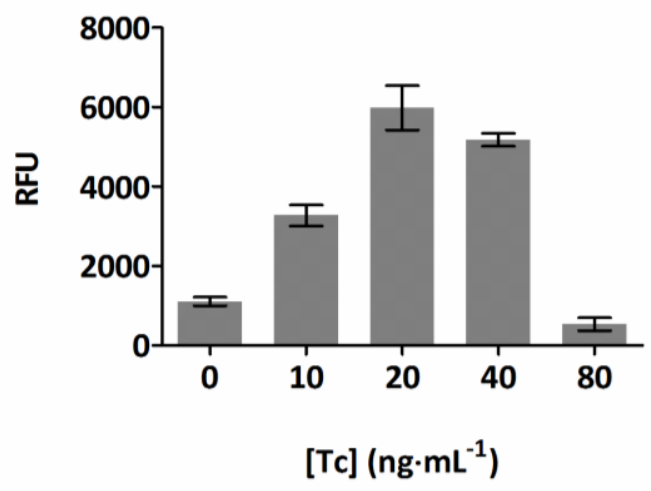

Figure 4.7: Optimal inducer concentration for MRC20. (A) Average endpoint growth relative to the untreated control, and (B) average endpoint RFU of the empty vector control strain MRC20 grown in different tetracycline (Tc) concentrations. Column heights represent mean values from three independent experiments with standard error bars shown.

The roles of the genes nudC and MSMEI_1905 in isoniazid sensitivity were further examined by overexpressing each gene in WT M. smegmatis $\mathrm{mc}^{2} 155$. Background sensitivity of WT M. smegmatis $\mathrm{mc}^{2} 155$ to isoniazid upon vector induction was determined by way of a dose-response assay against the empty vector control strain MRC20. A two-fold increase in the MIC of isoniazid was observed upon vector induction with $20 \mathrm{ng} \cdot \mathrm{mL}^{-1}$ tetracycline (Figure 4.8). Therefore for the genes of interest, only changes in the MIC of isoniazid that were greater than two-fold after induction were considered to be biologically significant.

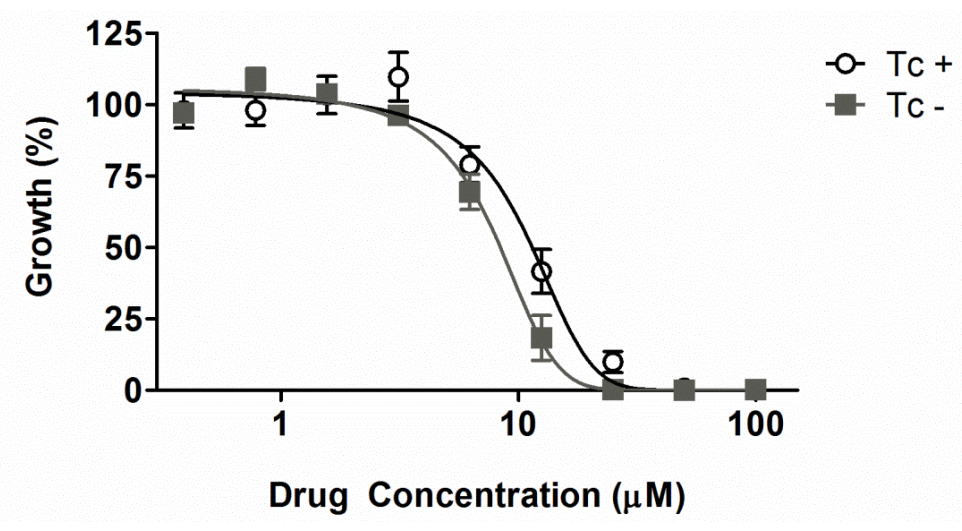

Figure 4.8: Dose response curve of MRC20 versus isoniazid \pm inducer. Data points represent the mean growth of the MRC20 strain with or without $20 \mathrm{ng} \cdot \mathrm{mL}^{-1}$ of the inducer tetracycline (Tc) over three independent experiments with standard error bars shown. 
The MICs of isoniazid for the nudC and MSMEI_1905 overexpression strains were determined by dose-response assay, along with the following control strains: an empty vector control, an inhA overexpression control, and an MSMEI_4267 random gene overexpression control (Figure 4.9). Overexpression of nudC and inhA resulted in a greater than 100 -fold increase in the MIC of isoniazid, from approximately $10 \mu \mathrm{M}$ in the un-induced overexpression strain, to greater than the highest tested concentration of $1 \mathrm{mM}$ in the induced overexpression strain. The MSMEI_1905 overexpression strain did not show any change in the MIC of isoniazid following induction, nor did the random gene expression control strain (MSMEI_4267).

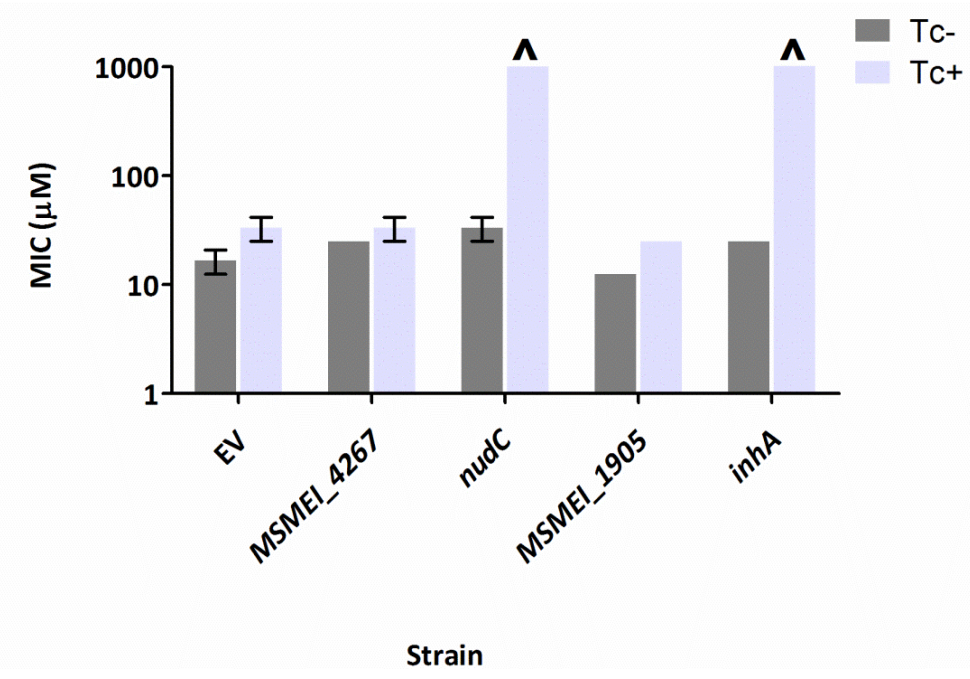

Figure 4.9: Overexpression strains versus isoniazid. MICs of isoniazid for gene overexpression strains including empty vector (EV) control MRC20, with and without induction with $20 \mathrm{ng} \cdot \mathrm{mL}^{-1}$ tetracycline (Tc). Column heights represent mean MICs over three independent experiments with standard error bars shown. For those columns that have no error bars the standard error was zero. The highest tested concentration of isoniazid was $1 \mathrm{mM}$, with MIC values greater than the highest tested concentration indicated with a caret (^).

\subsubsection{Rifampicin}

\subsubsection{Transposon insertion site identification}

The transposon insertion sites were able to be identified for 11 of the 12 mutants most hypersensitive to rifampicin identified in Chapter Three, using either inverse PCR or ligation-mediated PCR as described in section 4.2.1. (Table 4.3). 
Table 4.3: Transposon insertion sites in rifampicin hypersensitive mutants.

\begin{tabular}{|c|c|c|c|c|c|c|}
\hline $\begin{array}{l}\text { Mutant } \\
\text { Strain }\end{array}$ & Tn611 insertion location (ORF) & Putative Identification & $\begin{array}{l}\text { Common } \\
\text { Name }\end{array}$ & $\begin{array}{c}\text { Nearest } \\
\mathrm{H}_{37} \mathrm{Rv} \\
\text { homologue }\end{array}$ & $\begin{array}{c}\text { Gene } \\
\text { Length (bp) }\end{array}$ & POI \\
\hline myco0163 & MSMEI_6730 & proline-rich $28 \mathrm{kDa}$ antigen & $m t c 28$ & $R v 0040 c$ & 945 & 399 \\
\hline myco0891 & Undefined & Transposase & ISMsm2 & - & $987-1086$ & 97 \\
\hline myco1030 & MSMEI_6040 & penicillin binding protein & ponA2 & $R v 3682$ & 2454 & 260 \\
\hline myco2642 & $\begin{array}{l}19 \text { bp downstream of MSMEI_6263 } \\
>1000 \text { bp upstream of MSMEI_6264 }\end{array}$ & $\begin{array}{l}\text { MSMEI_6263 - ESX-1 transcriptional regulator } \\
\text { MSMEI_6264 - Conserved hypothetical protein }\end{array}$ & espR & $\begin{array}{c}R v 3849 \\
-\end{array}$ & $\begin{array}{l}405 \\
492\end{array}$ & - \\
\hline myco2694 & MSMEI_1186 & rifampicin ADP-ribosyl transferase & $a r r$ & - & 429 & 224 \\
\hline myco4005 & MSMEI_3093 & $\begin{array}{c}\text { ribosomal large subunit pseudouridine synthase } \\
\text { D }\end{array}$ & & $R v 1540$ & 930 & 528 \\
\hline myco5826 & $\begin{array}{c}98 \text { bp downstream of } M S M E I / 1800 \\
52 \text { bp upstream of } M S M E I \_1801\end{array}$ & $\begin{array}{c}\text { MSMEI_1800 - TetR like transcriptional regulator } \\
\text { MSMEI_1801 - Adenosylhomocysteinase }\end{array}$ & ahcY & $\begin{array}{l}R v 3249 C \\
R v 3248 C\end{array}$ & $\begin{array}{c}714 \\
1458\end{array}$ & - \\
\hline myco5883 & Undefined & Transposase & ISMsm4 & - & 1323 & 348 \\
\hline myco6762 & MSMEI_0227 & metallopeptidase & $z m p 1$ & $R v 0198 c$ & 2001 & 774 \\
\hline myco6859 & MSMEI_6301 & O-methyltransferase & omt & $R v 1153 c$ & 819 & 722 \\
\hline myco7011 & MSMEI_5621 & Cell envelope-related transcriptional attenuator & - & $R v 0822 c$ & 2379 & 502 \\
\hline
\end{tabular}

Rifampicin hypersensitive mutants are organised by their location in the mutant collection in ascending order. Transposon point of insertion (POI) is counted from the start of the gene. For the undefined mutant myco0891 the range of gene lengths of the four possible insertion sites is given. 
Two mutants, myco0891 and myco5883, contained Tn611 insertions in two different insertion elements, each of which is present in multiple copies within the genome of M. smegmatis $\mathrm{mc}^{2} 155$. Myco0891 contains a transposon insertion in an ISMsm2 transposase gene that occurs four times in the M. smegmatis $\mathrm{mc}^{2} 155$ genome at MSMEI_0054, MSMEI_3431, MSMEI_4411, and MSMEI_5518. Myco5883 contains a transposon insertion within an ISMsm4 transposase that occurs four times in the $M$. smegmatis $\mathrm{mc}^{2} 155$ genome at MSMEI_0419, MSMEI_1205, MSMEI_2757, and MSMEI_5245. The entire sequences generated for both of these mutants were within the ORFs of the transposase genes, therefore the specific copies of ISMsm2 or ISMsm4 that contained the insertions could not be determined by inverse PCR or ligation-mediated PCR.

\subsubsection{Complementation of rifampicin hypersensitive mutants}

The mutant strains selected for complementation, myco2694 and myco4005, were eight-fold and four-fold more sensitive to rifampicin respectively (Chapter Three) compared with a less than two-fold increase in sensitivity for the other mutant strains. It was expected that the greater sensitivity of these strains would make it easier to determine whether or not complementation was successful. The mutant strains myco2694 and myco4005 are henceforth referred to by the ORF containing the Tn611 insertion: Tn::arr and Tn::MSMEI_3093 respectively. Although vectors for the complementation of each strain were constructed, an empty vector control strain was not constructed for Tn::MSMEI_3093. No colonies grew when Tn::MSMEI_3093 was transformed with the empty vector pRC20, despite four experimental repeats. As there was no suitable control, complementation of Tn::MSMEI_3093 was not performed. Due to the already well characterised activity of Arr in M. smegmatis, the complementation of the Tn::arr mutant was to serve as a positive control for the complementation of rifampicin hypersensitive mutants. As such, complementation of Tn::arr was not pursued once it was decided to not pursue complementation of Tn::MSMEI_3093. 


\subsubsection{Overexpression of genes related to rifampicin hypersensitivity}

Background sensitivity of WT $M$. smegmatis to rifampicin upon vector induction was determined by way of a dose-response assay against the empty vector control strain MRC20. No change in the MIC of rifampicin for MRC20 was observed upon induction with $20 \mathrm{ng} \cdot \mathrm{mL}^{-1}$ tetracycline (Figure 4.10).

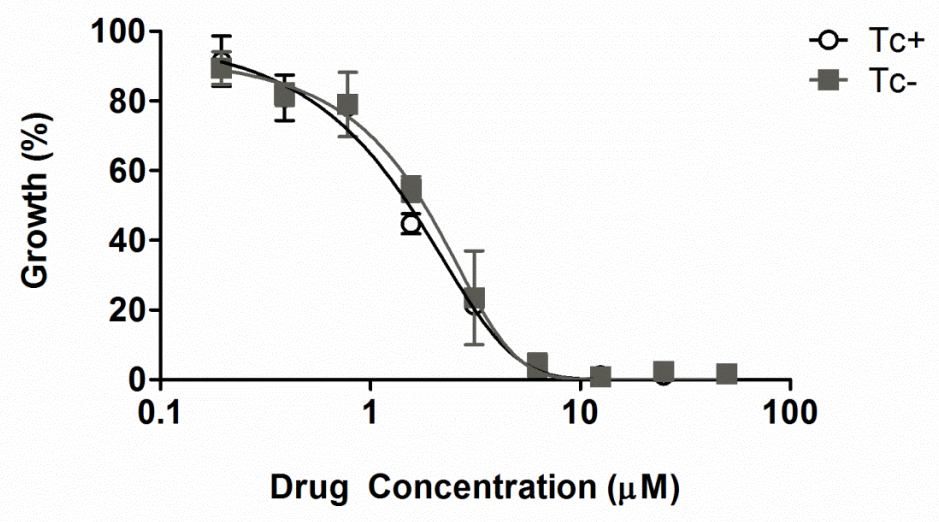

Figure 4.10: Dose response curve of MRC20 versus rifampicin \pm inducer. Data points represent the mean growth of the MRC20 strain with or without $20 \mathrm{ng} \cdot \mathrm{mL}^{-1}$ of the inducer tetracycline (Tc) over three independent experiments with standard error bars shown.

The role of MSMEI_3093 in rifampicin sensitivity was examined by overexpressing MSMEI_3093 in WT M. smegmatis $\mathrm{mc}^{2} 155$. The MIC of rifampicin for the empty vector control strain MRC20, the MSMEI_3093 overexpression strain, and the MSMEI_4267 random gene overexpression control strain were determined by a dose-response assay with and without induction with $20 \mathrm{ng} \cdot \mathrm{mL}^{-1}$ tetracycline (Figure 4.11). The MIC of rifampicin did not change for any of the three strains upon vector induction, with all strains showing an approximately equal MIC. 


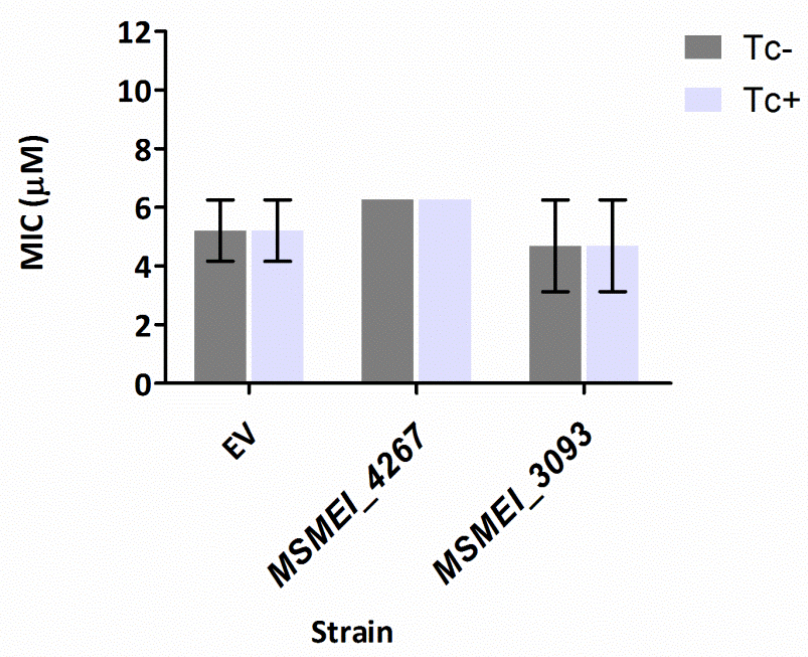

Figure 4.11: Overexpression strains versus rifampicin. MICs of rifampicin for gene overexpression strains including empty vector (EV) control MRC20, with and without induction with $20 \mathrm{ng} \cdot \mathrm{mL}^{-1}$ tetracycline (Tc). Column heights represent mean MICs over three independent experiments with standard error bars shown. For those columns that have no error bars the standard error was zero.

\subsubsection{Bedaquiline}

\subsubsection{Transposon insertion site identification}

The transposon insertion sites were able to be identified for eight of the 12 mutants most hypersensitive to bedaquiline identified in Chapter Three, using either inverse PCR or ligation-mediated PCR. Table 4.4 summarises the sequencing results. As mentioned in Chapter Three, the decision to pursue bedaquiline was made late into the thesis and due to time constraints the study of bedaquiline hypersensitive mutants did not progress to complementation or overexpression assays. 
Table 4.4: Transposon insertion site for bedaquiline hypersensitive mutants.

\begin{tabular}{|c|c|c|c|c|c|c|}
\hline $\begin{array}{l}\text { Mutant } \\
\text { Strain }\end{array}$ & Tn611 insertion location (ORF) & Putative Identification & $\begin{array}{l}\text { Common } \\
\text { Name }\end{array}$ & $\begin{array}{c}\text { Nearest } \mathrm{H}_{37} \mathrm{Rv} \\
\text { homologue }\end{array}$ & $\begin{array}{l}\text { Gene Length } \\
\text { (bp) }\end{array}$ & POI \\
\hline myco0934 & MSMEI_3416 & MmpL family protein & $M m p L 5$ & $R v 0676 c$ & 2910 & 1961 \\
\hline myco1994 & MSMEI_3189 & Glutamyl aminopeptidase & - & - & 1002 & 931 \\
\hline myco2176 & MSMEI_3358 & Esterase & - & $R v 1288$ & 1353 & 827 \\
\hline \multirow{2}{*}{ myco2833 } & 77 bp upstream of MSMEI_2671 & Biotin sulfoxide reductase & bisC & $R v 1442$ & 2292 & - \\
\hline & 181 bp upstream of MSMEI_2672 & Conserved hypothetical protein & - & $R v 2721 c$ & 2436 & - \\
\hline myco2908 & MSMEI_4815 & ATP synthase $F_{0}$ domain, $A$ subunit & atpB & $R v 1304$ & 777 & 563 \\
\hline myco3011 & MSMEI_4123 & Conserved hypothetical protein & yfiH & $R v 2149 c$ & 714 & 462 \\
\hline myco4393 & MSMEI_5136 & Transmembrane protein & - & $R v 1072$ & 837 & 770 \\
\hline myco5040 & MSMEI_5828 & PPE family protein & - & - & 1107 & 423 \\
\hline
\end{tabular}

Bedaquiline hypersensitive mutants are organised by their location in the mutant collection in ascending order. Transposon point of insertion (POI) is counted from the start of the gene. 


\subsection{Discussion}

\subsubsection{Identification of transposon insertion sites}

Two different techniques, inverse PCR and ligation-mediated PCR, were used in the identification of the Tn611 insertion sites in the drug hypersensitive mutants. Both inverse PCR and ligation-mediated PCR rely upon endonuclease digestion of the transposon mutant DNA to generate fragments that contain both the end of the transposon, and the adjacent gDNA into which it has inserted. Inverse PCR was initially used for identifying the Tn611 insertion site using Rsal digestion as described by Billman-Jacobe et al. (2006). In this study inverse PCR was unable to generate the product containing the Tn611 end and the flanking gDNA for some of the mutant strains, amplifying only the internal transposon product. Additionally, in some cases a PCR product was generated but the end of the transposon was too close to an Rsal site resulting in very short fragments from which no reliable sequence matches could be made. In order to overcome this, the inverse PCR method was repeated using Eagl digestion for those mutant strains where Rsal based inverse PCR failed. Inverse PCR using these Eagl digested samples enabled identification of the Tn611 insertion site for several more, but not all of the transposon mutants, likely due to the same reasons as the failure of Rsal based inverse PCR. Ligation-mediated PCR was used as an alternative method for identifying the mutation site for those mutant strains where inverse PCR using either restriction enzyme was unsuccessful. Ligation-mediated PCR utilises Sall digestion to generate DNA fragments containing the transposon DNA/gDNA junction, and a DNA linker to allow selective amplification of this junction. Utilisation of ligation-mediated PCR resulted in identification of the mutation site for the majority of mutants where inverse PCR was unsuccessful. However, the mutation sites could not be identified for one rifampicin hypersensitive mutant and for five of the bedaquiline hypersensitive mutants, using either inverse PCR or ligation-mediated PCR due to an inability to generate PCR products for sequencing. The reason for the inability to generate products for these mutant strains is unknown, although due to the late inclusion of bedaquiline in the study significantly less time was spent troubleshooting the identification of Tn611 insertion in the bedaquiline hypersensitive mutants than those for isoniazid and rifampicin. Future investigations utilising other methods such as ligating the DNA fragments containing the gDNA/transposon DNA 
junction into a vector and sub-cloning into E.coli (Billman-Jacobe et al., 2006) may yield more information on the transposon insertion site for these remaining mutants.

\subsubsection{Genetic complementation}

The three mutants most sensitive to isoniazid and two mutants most sensitive to rifampicin as determined in Chapter Three were selected for complementation. These particular mutants were selected for complementation as they showed a large change in the MIC of isoniazid or rifampicin than the remainder of the 12 most sensitive mutants. These mutants were prioritised for complementation as it was expected that the large decrease in the MIC of the relevant drug for these mutants would make it easier to determine whether or not complementation was successful.

Complementation was performed for the isoniazid hypersensitive mutant strains $T n:: n u d C$ and Tn::MSMEI_1905. Complementation of a third isoniazid hypersensitive mutant, Tn::MSMEI_6092, was unable to be completed due to deletions that occurred in all of the MSMEI_6092 expression constructs that did not contain the gene in the reverse orientation. The deletions that occurred in the MSMEI_6092 construct may have been due to the expression of this gene being toxic to E. coli. Although expression of MSMEI_6092 was not induced when the vector was sub-cloned, the tetracycline inducible promoter of pRC20 is leaky in E. coli $\mathrm{DH} 5 \alpha$ in which the vector sub-cloning was performed.

Additionally, although a complementation strain was able to be constructed for the rifampicin hypersensitive mutant Tn::MSMEI_3093, no empty vector control strain could be built (section 4.3.2.2). The mutant Tn::MSMEI_3093 contains the Tn611 insertion within the ribosomal large subunit pseudouridine synthase gene and therefore may have defective translation. This mutant displayed a slow growth phenotype and it is possible that the addition of the hygromycin B antibiotic, an aminoglycoside and inhibitor of transcription, required to maintain the plasmid within the host, prevented the strain Tn::MSMEI_3093 containing pRC20 from growing. Interestingly, the ability to generate the Tn::MSMEI_3093 complementation strain suggests that it was possible to complement whatever was preventing the generation of the empty vector control e.g. hygromycin sensitivity. However, this was not examined further in the present study. As no empty vector control could be 
built for Tn::MSMEI_3093 complementation of this strain was not completed. Consequently, it was decided to not attempt complementation of Tn::arr by itself, as the activity of Arr in M. smegmatis is already well characterised (Alexander et al., 2003; Dabbs et al., 1995; Quan et al., 1999), and the complementation of Tn::arr was only to serve as a control for the complementation of rifampicin hypersensitive mutants.

\subsubsection{Gene overexpression}

To further characterise the genetic basis of drug hypersensitivity for mutants hypersensitive to isoniazid (Tn::nudC and Tn::MSMEI_1905), and rifampicin (Tn::MSMEI_3093) the genes containing the Tn611 insertions were overexpressed in WT M. smegmatis $\mathrm{mc}^{2} 155$. It was found that MRC20 in the presence of the inducer tetracycline had an approximate two-fold increase in the MIC of isoniazid, while no change in the MIC of rifampicin was observed. The reason for the increased isoniazid MIC observed upon vector induction is unknown. Further investigation into the interaction between isoniazid and tetracycline may reveal whether there is an overlap between the modes of action of the two drugs or whether the expression of GFP influences susceptibility to isoniazid.

\subsubsection{Polar effects of Tn611 insertion}

At least six of the hypersensitive transposon mutants contained the Tn611 insertion within an intergenic region, suggesting that Tn611 exerts a polar effect on the genes surrounding the insertion site. A polar effect also appears responsible for the isoniazid hypersensitive phenotype of the Tn::MSMEI_1905 mutant as complementation of this mutant with MSMEI_1905 failed to recover WT isoniazid sensitivity. Interestingly, the gene that is adjacent to MSMEI_1905 is nudC, which is known to be involved in isoniazid detoxification.

Therefore, although the most likely candidate genes responsible for the drug hypersensitivity phenotype are those that contain a transposon insertion, it is possible that polar effects on the adjacent genes also play a role. As complementation was not performed for all of the most sensitive mutants, it is not possible to say with certainty which gene is responsible for the sensitivity phenotype for the remaining hypersensitive mutants 
identified in this study. Further investigations into the effect of complementing the remaining hypersensitive mutants may reveal the extent that polar effects of Tn611 play a role in drug hypersensitivity.

\subsubsection{Genotypes related to isoniazid hypersensitivity}

\subsubsection{Links to known isoniazid mode of action and resistance mechanisms}

Four of the 12 mutants most hypersensitive to isoniazid, as determined in Chapter Three, had a transposon insertion in or adjacent to genetic loci connected to aspects of the known mode of action and/or resistance mechanisms for isoniazid. These genes were: nudC, which encodes the NADH pyrophosphatase NudC (two mutants: Tn::nudC and Tn::MSMEI_1905); $e c c \mathrm{Ca}_{1}$, the ESX-1 conserved component $\mathrm{Ca}$; and the $\operatorname{ahpCD}$ operon, which encodes the heterodimeric alkylhydro peroxide reductase AhpCD. The identification of these four mutants with known links to the mode of action of isoniazid validates the use of the assays in Chapter Three for investigating isoniazid mode of action.

NudC (MSMEI_1904) is known to be involved in isoniazid detoxification in M. smegmatis $\mathrm{mc}^{2} 155$ and $M$. bovis BCG (Wang et al., 2011). Specifically, NudC has been shown to hydrolyse the INH-NAD adduct, which is the active form of isoniazid. The mutant Tn::nudC generated in this thesis contains a transposon insertion within the ORF of nudC, and likely represents a loss of function mutation. Interestingly, the mutant Tn::MSMEI_1905 contained a Tn611 insertion in the gene MSMEI_1905 which is immediately adjacent to the nudC gene in $M$. smegmatis $\mathrm{mc}^{2} 155$. Complementation experiments showed that expression of nudC was able to complement the isoniazid hypersensitive phenotype measured in the Tn::nudC mutant, while expression of MSMEI_1905 had no effect on the isoniazid hypersensitivity of the Tn::MSMEI_1905 mutant. Therefore, it is possible that the isoniazid hypersensitivity of the Tn::MSMEI_1905 mutant was due to a polar effect on the expression of the adjacent nudC gene. Further investigation into this, such as measuring nudC gene expression levels using qPCR, would reveal whether nudC expression levels are lower in the Tn::MSMEI_1905 mutant than in WT M. smegmatis. To further characterise the role of nudC and MSMEI_1905 in isoniazid sensitivity each gene was overexpressed in WT M. smegmatis as described in 
section 4.3.1.3. Overexpression of nudC resulted in increased resistance to isoniazid, consistent with the complementation experiments. These results corroborate those published by Wang et al. (2011), and strongly support the role of NudC in the detoxification of the INH-NAD adduct. Overexpression of MSMEI_1905 did not alter sensitivity to isoniazid, again consistent with the results from the complementation experiments. The overexpression results further confirm that MSMEI_1905 was not responsible for the isoniazid hypersensitive phenotype in this mutant strain.

Myco2659 contained an insertion in the ESX-1 conserved component $\mathrm{Ca}_{1}$ (eccCa, MSMEI_0062) which is also known to be involved in the mode of action of isoniazid. ESX-1 is a type-VII secretion system that plays an important role in excreting proteins involved in virulence in mycobacterial species (Pym et al., 2002, 2003). It is loss of the ESX-1 locus, which is located in the region of difference 1 (RD1), that is partly responsible for the attenuation of the M. bovis BCG vaccine strain (Brosch et al., 2007; Pym et al., 2002, 2003). It has previously been shown that EccCa 1 is required for proper function of the ESX-1 system, and that loss of function mutants of $e c c \mathrm{Ca}_{1}$ in a related mycobacterial species, M. marinum, exhibit reduced secretion of ESAT-6, a protein secreted by the ESX-1 machinery (Joshi et al., 2012). In addition, EccCa1 interacts with components of the cell wall biosynthesis machinery, including components of the FASII system which is the pathway inhibited by isoniazid (Joshi et al., 2012). Joshi et al. (2012) demonstrated that $T n:: e c c C a_{1}$ mutants produced $30 \%-40 \%$ less total mycolic acid compared to WT, suggesting that EccCa 1 stabilizes mycolic acid biosynthesis. Interestingly, $T n:: e c c C a_{1}$ mutants were also more sensitive to ethionamide, an analogue of isoniazid that also inhibits InhA. Therefore, it is likely that the $T n:: e c c C a_{1}$ mutant (myco2659) identified in this thesis is hypersensitive to isoniazid due to a partially inhibited mycolic-acid biosynthesis pathway resulting from an absence of functional EccCa1.

The third genetic locus related to the mode of action of isoniazid that was identified in this thesis was the $a h p C D$ operon, with the mutant myco7102 containing a Tn611 insertion 36 bp downstream of the $a h p C D$ operon (MSMEI_4765/4766). The proteins AhpC and AhpD together function as a heterodimeric alkylhydro peroxidase that protects mycobacteria from oxidative damage induced by alkylhydro peroxides (Chen et al., 1998). M. tuberculosis isoniazid resistant katG mutants often have reduced catalase activity resulting in increased oxidative stress. $A h p C D$ is up regulated in $M$. tuberculosis katG mutants, where it 
compensates for the reduced KatG activity and resulting increased oxidative stress (Dhandayuthapani et al., 1996; Sherman et al., 1996). Overexpression of AhpCD has been shown to increase resistance to isoniazid, while conversely, loss of function ahpCD mutants appear to show increased sensitivity to isoniazid, due to an altered oxidative stress response and concomitant altered katG expression (Zhang et al., 1996). Although myco7102 does not contain a Tn611 insertion within the $a h p C$ or $a h p D$ ORFs, its close proximity likely has a polar effect upon the expression of the $a h p C D$ operon resulting in the observed isoniazid hypersensitivity. This could be confirmed by quantitative PCR to measure the expression of ahpCD in the myco7102 mutant.

\subsubsection{Identification of potentially novel aspects of isoniazid's mode of action}

Other isoniazid hypersensitive mutants identified in this thesis were found to contain $\operatorname{Tn} 611$ insertions in genes that represent potentially novel aspects to the aforementioned known modes of action. Other mutants contain Tn611 insertions in genes that have no known links to the known mode of action of isoniazid, and represent either novel aspects of the known mode of action, or aspects of novel modes of action.

Tn::MSMEI_6092 contained a Tn611 insertion in a gene encoding a Fur-like protein (MSMEI_6092). There are two classes of Fur proteins, Fur A and Fur B, and MSMEI_6092 was determined to be more closely related to the FurA type proteins. Fur like regulators regulate various aspects of the interlinked process of iron metabolism and the oxidative stress response (Dussurget and Smith, 1998). FurA is the regulator for expression of KatG, a catalase/peroxidase responsible for detoxification of hydrogen peroxides, and the activation of isoniazid. M. smegmatis $\mathrm{mc}^{2} 155$ has two copies of $k a t G$, each with its own furA regulator immediately upstream of the katG ORF. The furA like gene encoded by MSMEI_6092 is not near a katG, or katG like gene, and has no direct homolog in $M$. tuberculosis. As an expression construct for MSMEI_6092 could not be constructed in this thesis it was not possible to complement the Tn::MSMEI_6092 strain and confirm if the mutation of this gene was in fact responsible for the isoniazid hypersensitivity of this strain. It is possible that the Tn611 insertion in Tn::MSMEI_6092 had a polar effect on a nearby gene, however the known 
link between the oxidative stress response, isoniazid, and the pro-drug activating role of KatG, strongly suggests it is loss of function of the MSMEI_6092 gene product that results in the isoniazid hypersensitivity phenotype of this strain. The furA like gene encoded by MSMEI_6092 may have a regulatory role for one or both of the katG genes in M. smegmatis. Measurement of katG expression levels in the Tn::MSMEI_6092 mutant would reveal the relationship between this furA like gene and KatG. Alternatively, MSMEI_6092 may regulate other components of the oxidative stress response, indirectly effecting katG expression.

Myco1897 and myco6482 contained transposon insertions within transcriptional regulators that have homologs in other bacterial species that are involved in multi-drug resistant phenotypes. Myco1897 contained an insertion in a multiple antibiotic resistance (Mar) type regulator (MarR, MSMEI_5538). In E. coli MarR mediates multiple antibiotic resistance through regulation of the genes marA and $\operatorname{mar} B$ (Cohen et al., 1993), for which mycobacteria lack obvious homologs. Interestingly, expression of E. coli marA in M. smegmatis has been shown to increase resistance to a range of antibiotics including isoniazid (McDermott et al., 1998). A MarRAB system was recently characterised in M. smegmatis that MSMEI_5538 was not a part of, and which showed no role in isoniazid sensitivity (Zhang et al., 2014). This suggests that $M$. smegmatis may have more than one MarRAB system, perhaps with different substrate specificities.

Myco6482 contained a transposon insertion in MSMEI_5460, which encodes a putative transcriptional regulator of unknown function, and has no clear homolog in $M$. tuberculosis. Bowman and Ghosh (2014) found that expression of MSMEI_5460 was up-regulated in a multi-drug resistant KO mutant of MSMEI_5969, which encodes an anti-sigma factor proposed to be involved in regulating the 28 sigma factors in $M$. smegmatis. It was proposed that up-regulation of genes including MSMEI_5460 was responsible for the observed multi-drug resistant phenotype of this KO strain. The role of these transcriptional regulators in a multi-drug resistance phenotype could be further investigated by examining the cross-sensitivity of these strains to other antibiotics, and determining which genes they regulate.

Myco2100 and myco3113 contained Tn611 insertions within gene clusters that appear to be involved in sulfur metabolism. Altered sulfur metabolism is linked to a deficient oxidative 
stress response (Dussurget and Smith, 1998). These strains may compensate for the increased level of basal oxidative stress by increasing katG expression, resulting in an increasing sensitivity to isoniazid as in AhpCD mutants of mycobacteria (Dhandayuthapani et al., 1996; Sherman et al., 1996). Further investigation into the expression levels of katG in myco2100 and myco3113 may reveal whether this is the case.

Myco0409 contained an insertion within $\arg D$, which lies in the middle of the arginine biosynthesis cluster. Because of this genetic organisation, even if the cause of isoniazid sensitivity in this strain is not due to a mutation in $\arg D$ specifically but due to a polar effect, it is very likely that arginine biosynthesis would be disrupted. Almost all of the genes in the arginine biosynthesis cluster have been shown to be essential for in vitro growth of M. tuberculosis $\mathrm{H}_{37} \mathrm{Rv}$ by transposon based mutagenesis (Sassetti et al., 2003), with the exception of $\operatorname{argR}$, which is a negative regulator of arginine biosynthesis (Maas, 1994). To the best of my knowledge, there has not been any literature published examining a link between arginine metabolism and isoniazid activity. In addition, arginine biosynthesis is not known to be linked to the oxidative stress pathway. Therefore, these results may represent a novel aspect of the mode of action of isoniazid and a novel role for the arginine biosynthesis machinery.

Myco6166 contained a transposon insertion in a conserved hypothetical protein with predicted helicase and DNA binding activity (MSMEI_5554). MSMEI_5554 likely encodes a transcriptional regulatory protein and may regulate genes involved in the isoniazid mode of action, such as oxidative stress response genes. However, the $\mathrm{H}_{37} \mathrm{Rv}$ homolog $\mathrm{Rv} 0862 \mathrm{c}$ has no known reported function so the role of MSMEI_5554 remains unclear.

Myco6192 contains a transposon insertion within the potassium transporter encoding gene $k d p A$. The $\mathrm{KdpA}$ protein functions in a complex with $\mathrm{KdpB}$ and $\mathrm{KdpC}(\mathrm{KdpABC})$ as a potassium transporter, and is stabilised by the co-transcribed gene product KdpF (Gannoun-Zaki et al., 2013). Overexpression of KdpF caused altered cell morphology in $M$. bovis BCG in a study by Gannoun-Zaki et al., (2013). Although, no change in isoniazid resistance was seen when KdpF was overexpressed; however, investigation into altered resistance in $\mathrm{KdpF}$ loss of function mutants was not performed. 


\subsubsection{Genotypes related to rifampicin hypersensitivity}

\subsubsection{Links to known rifampicin mode of action and resistance mechanisms}

After sequencing the Tn611 insertion sites of 11 of the 12 mutants most hypersensitive to rifampicin, as determined in Chapter Three, it was found that three mutants had a connection to known rifampicin resistance mechanisms. These three mutants had Tn611 insertions in arr, which encodes the rifampicin ADP-ribosyl transferase Arr, and ponA2, which encodes the penicillin binding protein PonA2. The third mutant had an insertion in the gene adjacent to the lipoprotein signal peptidase IspA. The identification of these three mutants with known links to the mode of action of rifampicin validates the use of the assay used in Chapter Three for investigating rifampicin mode of action.

Arr is involved in a known mechanism of rifampicin inactivation in M. smegmatis, whereby Arr attaches a ribose moiety to rifampicin resulting in loss of activity (Dabbs et al., 1995; Quan et al., 1999; Tanaka et al., 1996). A Tn::arr mutant of M. smegmatis has previously been identified in an assay for hypersensitive rifampicin transposon mutants (Alexander et al., 2003). As the activity of arr in WT M. smegmatis is already well characterised, complementation of the Tn::arr mutant, and overexpression of arr in WT M. smegmatis were not performed in this thesis.

The second mutant with known links to the mode of action of rifampicin was myco1030, which contained a transposon insertion in ponA2, a penicillin binding protein. PonA2 is suspected to play a role in altering peptidoglycan cross-linking in the mycobacterial cell wall during the switch to stationary phase in M. tuberculosis (Vandal et al., 2009). Vandal et al. (2009), showed that a M. tuberculosis $\mathrm{H}_{37} \mathrm{Rv}$ Tn::ponA2 mutant had slightly increased sensitivity to rifampicin and other lipophilic drugs, but no increased sensitivity to non-lipophilic drugs such as isoniazid. A ponA2 KO mutant of M. smegmatis also previously demonstrated increased sensitivity to rifampicin, along with reduced stationary-phase survival and an altered cell wall (Patru and Pavelka, 2010). The findings from these previous studies suggest that the sensitivity of the Tn::ponA2 mutant identified in this thesis is not rifampicin specific; however, this was not further investigated in this thesis. 
Myco4005 contained a transposon insertion in a gene encoding the ribosomal large subunit pseudouridine synthase D (MSMEI_3093). M. smegmatis $\mathrm{mc}^{2} 155$ MSMEI_3092 and MSMEI_3093 share the same genetic organisation as the $\mathrm{H}_{37} \mathrm{Rv}$ homologs, which are co-transcribed. Therefore, MSMEI_3093 is likely also co-transcribed with MSMEI_3092, which encodes the lipoprotein signal peptidase IspA. A previous study demonstrated that overexpression of $I s p A$ in $\mathrm{H}_{37} \mathrm{Rv}$ leads to increased resistance to rifampicin (Pathak, 2013). Interestingly, Pathak (2013) also showed that overexpression of IspA also led to increased resistance to isoniazid in $M$. tuberculosis. In this thesis it was found that overexpression of MSMEI_3093 resulted in no change in the MIC of rifampicin, which may indicate that a polar effect on IspA is the genotypic cause for the rifampicin hypersensitivity observed in this strain. Complementation with MSMEI_3093 would determine the role of this gene in the rifampicin hypersensitivity of the Tn::MSMEI_3093 strain. Complementation of this strain was not performed in this study as no suitable empty vector control could be constructed. Future complementation of this strain with MSMEI_3093 and IspA would allow the gene responsible for hypersensitivity to be determined.

\subsubsection{Identification of potentially novel aspects of rifampicin's mode of action}

The remaining rifampicin hypersensitive mutants that the Tn611 insertion site was identified for have either tentative links, or no clear links to the known mode of action and/or detoxification mechanisms for rifampicin, and represent potentially novel modes of action or detoxification mechanisms.

Myco0163 contained a transposon insertion within $m t c 28$, which encodes a $28 \mathrm{kDa}$ secreted antigen. Mtc28 contains large proline rich regions that have been implicated in modulating the interaction between penicillin binding proteins (PBP) and peptidoglycan (PG) metabolism (Manca et al., 1997; Patru and Pavelka, 2010). Therefore, as with the ponA2 mutant, the hypersensitivity of this strain may not be specific to rifampicin; however, this was not further investigated in this thesis.

The mutant myco2642 contained a Tn611 insertion 19 bp downstream of the ESX-1 regulator EspR (MSMEI_6263), and over 1000 bp upstream of MSMEI_6264. EspR drives expression of 
ESX-1 genes, increasing pump activity (Raghavan et al., 2008). As discussed in section 4.4.5.1, the ESX-1 system is involved in secretion of proteins that are important for mycobacterial virulence. The transposon mutant myco2642 is predicted to have a loss of activity of the $\operatorname{esp} R$ gene product and therefore decreased ESX-1 activity. To the best of my knowledge, no links between a defect in EspR and/or the ESX-1 system and rifampicin sensitivity exist within the literature. As ESX-1 is involved in pumping out many substrates the specific mechanism for rifampicin hypersensitivity in myco2642 is unknown, however it represents a potentially novel role for EspR, and by association ESX-1, in the rifampicin mode of action, or its detoxification.

Myco5826 contains a transposon insertion $98 \mathrm{bp}$ downstream of a gene encoding a predicted TetR like transcriptional regulator (MSMEI_1800). To the best of my knowledge no links between the rifampicin mode of action and this gene, or the M. tuberculosis homolog (Rv3249c), exist in the literature. Identification of the genes regulated by this TetR transcriptional regulator would facilitate understanding the basis for the rifampicin hypersensitivity of this strain

Myco6762 contains a transposon insertion in the gene encoding the metallopeptidase Zmp1 (MSMEI_0227). The M. tuberculosis Zmp1 homolog has been shown to be involved in pathogenicity whereby Zmp1 prevents formation of the inflammasome, a protein complex involved in modulating the host response to M. tuberculosis infection (Ferraris and Rizzi, 2011; Ferraris et al., 2011; Master et al., 2008). No links between this process and the mode of action of rifampicin could be identified in the literature so the mechanism of rifampicin hypersensitivity in myco6762 remains unknown.

Myco6859 contains a Tn611 insertion in MSMEI_6301, which encodes the O-methyltransferase (omt). O-methyltransferase is involved in methylation of the cell wall of M. smegmatis (Jeevarajah et al., 2002). No link between omt or the M. tuberculosis homolog $R v 1153 c$, and altered rifampicin sensitivity could be found in the literature.

Myco7011 contained a Tn611 insertion in a gene encoding a cell envelope-related transcriptional attenuator (MSMEI_5621). The M. tuberculosis homolog, Rv0822c, has been shown to be significantly up-regulated in an RD1 deletion mutant of $\mathrm{H}_{37} \mathrm{Rv}$ (Mostowy et al., 2004). RD1 is one of the major regions of the genome of M. bovis BCG that is missing 
compared to the genome of $M$. bovis and $M$. tuberculosis. The absence of RD1 has been shown to be largely responsible for the attenuation of BCG, due to the loss of the ESX-1 type-VII secretion system. The relationship of this gene to the RD1 locus suggests this gene may be involved in the regulation of ESX-1, similar to the rifampicin hypersensitive Tn::espR mutant also identified in this thesis. Therefore, these results strongly implicate a role for ESX-1 in the rifampicin mode of action or detoxification.

\subsubsection{Genotypes related to bedaquiline hypersensitivity}

\subsubsection{Links to known bedaquiline mode of action and resistance mechanisms}

Characterisation of the Tn611 insertion sites of the 12 mutants most hypersensitive to bedaquiline as determined in Chapter Three, revealed that two mutants had connections to known aspects of the mode of action and/or resistance mechanisms for bedaquiline These two mutants had Tn611 insertions in $\operatorname{atpB}$, which encodes the ATP synthase $F_{0}$ domain A subunit, and $m m p L 5$, which encodes a transmembrane transporter. The identification of these two mutants with Tn611 insertions within or adjacent to genes with known links to the mode of action of bedaquiline validates the use of the assays used in Chapter Three for investigating bedaquiline mode of action.

Myco2908 contained a Tn611 insertion in the atpB gene, which encodes subunit A of the ATP synthase $F_{0}$ domain. The F-ATP synthase consists of two domains, $F_{0}$ and $F_{1}$, which function together to generate ATP. The $F_{0}$ unit is composed of the $A, B$, and $C$ subunits and the known target of bedaquiline is the $C$ subunit of the ATP synthase $F_{0}$ domain encoded by atpE (Andries et al., 2005; Koul et al., 2007). In M. tuberculosis, atpB and atpE are co-transcribed as an operon; therefore, the $\operatorname{Tn}:: a t p B$ mutant identified in this thesis may also have altered atpE expression (Roback et al., 2007; Sala et al., 2009). Interestingly the $\mathrm{H}_{37} \mathrm{Rv}$ homolog of $a t p B$ has been shown to be essential in $M$. tuberculosis by transposon mutagenesis (Griffin et al., 2011; Sassetti et al., 2003). The requirement for atpB in M. tuberculosis for viability, suggests that there is some functional redundancy in M. smegmatis as a viable Tn::atpB mutant was generated in this thesis. The bedaquiline hypersensitivity of this mutant may be due to a general defect in the ATP synthase, or more 
specifically due to altered expression of the target of bedaquiline atpE; however, this was not further investigated in this thesis.

The second mutant with links to the known mode of action of bedaquiline was myco0934, which contained a Tn611 insertion in mmpL5 (MSMEI_3416). The homologous M. tuberculosis protein is an efflux pump that is proposed to pump bedaquiline out of the cell. Increased expression of $m m p L 5$ in $\mathrm{H}_{37} \mathrm{Rv}$ was found to increase bedaquiline resistance (Andries et al., 2014; Hartkoorn et al., 2014). The mutant Tn::mmpL5 identified in this study therefore likely has increased bedaquiline sensitivity due to a loss of MmpL5 efflux activity, resulting in increased intracellular concentrations of bedaquiline.

\subsubsection{Identification of potentially novel aspects of bedaquiline's mode of action}

The remaining bedaquiline hypersensitive mutants that were sequenced were found to contain Tn611 insertions in genes with no link to the known mode of action of bedaquiline. These therefore represent potentially novel aspects of the mode of action or detoxification mechanisms of bedaquiline.

Myco1994 contains a transposon insertion in MSMEI_3189, which encodes a M42 type glutamyl aminopeptidase for which there is no apparent $M$. tuberculosis homolog. No information in the literature relating this gene to any aspects of the bedaquiline mode of action could be found, therefore, the mechanism of bedaquiline hypersensitivity for this mutant remains unclear.

Myco2833 contains a transposon insertion $77 \mathrm{bp}$ upstream of the gene encoding the biotin sulfoxide reductase (bisC, MSMEI_2671), and 181 bp upstream of MSMEI_2672, which encodes a conserved hypothetical protein. As discussed previously, Tn611 exerts a polar effect on nearby genes and therefore it is possible that the Tn611 insertion in myco2833 is disrupting the function of bisC or MSMEI_2672. Protein BLAST results suggest the product of MSMEI_2672 is involved in cell wall biosynthesis. The gene product of MSMEI_2672 contains a conserved region consisting of four repeats of a 54 aa sequence named LGFP. The LGFP repeats are proposed to be involved in anchoring the protein to the cell wall, with these 
repeats found in proteins required for stabilising the cell wall in Methanosarcina acetivorans (Adindla et al., 2004). Alternatively, the Tn611 insertion in myco2833 may be causing hypersensitivity to bedaquiline by a polar effect on MSMEI_2671 gene expression, although the exact mechanism of sensitivity is unclear. Generation of knock out mutants of each gene would determine which gene is responsible for the bedaquiline hypersensitivity of this strain.

Myco3011 contained a transposon insertion within the gene MSMEI_4123, which encodes a conserved hypothetical gene named $y f i H$. The genetic neighbourhood of $y f i H$ includes a number of genes involved in cell division including $f t z Q$ and $f t s Z$, a genetic organisation conserved in M. tuberculosis $\mathrm{H}_{37} \mathrm{Rv}$ (Doerks et al., 2012; Weiling Hong et al., 2013). The conserved genetic region within which $y f i H$ resides suggests that $\mathrm{YfiH}$ is involved in the process of cell division and/or cell wall maintenance, similar to its genetic neighbours. Therefore, the bedaquiline hypersensitivity of this strain may be due to deficient cell wall division/maintenance.

Myco4393 contained a transposon insertion in MSMEI_5136, which encodes a putative transmembrane protein. Both MSMEI_5136, and its M. tuberculosis homolog Rv1072, reside in a relatively conserved genomic region encoding genes related to fatty acid metabolism. Interestingly, $R v 1072$ has been identified as a gene required for late stage survival of mycobacteria in a guniea pig infection model, as well as being up-regulated during oxidative stress and nutrient limitation (Betts et al., 2002; Kaushal et al., 2002; Manganelli et al., 2002; Schnappinger et al., 2003).

Myco5040 contains a transposon insertion within the gene MSMEI_5828, which encodes a PPE family protein that contains the characteristic $\sim 180$ bp proline-proline-glutamic acid motif at the N-terminus. Little is known about the function of PPE proteins, which together with PE proteins (with a characteristic $100 \mathrm{bp}$ proline-glutmaic acid $\mathrm{N}$-terminal motif) comprise $10 \%$ of the $M$. tuberculosis genome; The $M$. smegmatis genome only contains four PPE genes (Nair et al., 2009). Adjacent to MSMEI_5828 is MSMEI_5829, which encodes a lipid transfer protein Itp2, and is part of a conserved operon spanning MSMEI_5829 to MSMEI_5834 in M. smegmatis, and the homologs Rv3540c to Rv3545c in M. tuberculosis $\mathrm{H}_{37} \mathrm{Rv}$. This conserved region has been shown to be involved in cholesterol metabolism in 
both M. smegmatis and M. tuberculosis (García-Fernández et al., 2013; Uhía et al., 2012). This operon is likely defective in the myco5040 mutant due to polar effects of the Tn611 insertion, which would lead to dysfunctional cholesterol metabolism. Therefore, a defective cholesterol metabolism may be responsible for the bedaquiline hypersensitivity of this strain, and may represent a novel aspect of the mode of action of bedaquiline. To the best of my knowledge, no clear links between cholesterol metabolism and bedaquiline exist in the literature.

\subsubsection{Summary}

In summary, the Tn611 insertion site for 31 drug hypersensitive mutants of $M$. smegmatis were identified in this chapter; 12 isoniazid hypersensitive strains, 11 rifampicin hypersensitive strains, and 8 bedaquiline hypersensitive strains. Of the isoniazid hypersensitive mutants, four had links to either the known mode of action or detoxification pathways of isoniazid: $a h p C$, eccCa1, and nudC which was identified twice with two separate mutants. Of the rifampicin hypersensitive mutants, two had connections to either the known mode of action or detoxification pathways of rifampicin: arr and ponA2. Of the bedaquiline hypersensitive mutants, two mutants had links to either the known mode of action or detoxification pathways of bedaquiline: $\operatorname{atp} B$ and $m m p L 5$. Collectively, these results validate the use of the assay used in this thesis for identifying genes related to drug mode of action.

The remaining 23 mutants found to be hypersensitive to isoniazid, rifampicin, or bedaquiline that the insertion sites were identified for had no clear links to the known mode of action or detoxification pathways for each drug, and therefore represent novel aspects of either drug mode of action or detoxification mechanisms. As complementation of the transposon insertion was not attempted for the majority of drug hypersensitive mutants validated in Chapter Three, it is not possible to definitively identify the gene responsible for the observed drug hypersensitivity. However, for a number of strains the genes containing or adjacent to the Tn611 insertion site provide promising links to the mode of action of isoniazid, rifampicin, and bedaquiline respectively. Specifically, three isoniazid hypersensitive mutants contained Tn611 insertions either within or adjacent to genes linked to the oxidative stress response, which is known to interact with isoniazid via KatG. Interestingly, two genes related to the type-VII secretion systems ESX-1 were identified as containing Tn611 insertions in 
rifampicin hypersensitive mutants. To the best of my knowledge no link between rifampicin and the ESX-1 locus exists in the literature. There were no such apparent trends in the bedaquiline hypersensitive mutants; however, this is likely a limitation of the number of mutants sequenced for each compound. Had more drug hypersensitive mutants had their Tn611 insertion sites identified, more trends in terms of pathways containing Tn611 insertions may have become apparent. 


\section{Characterising drug sensitivity phenotypes of Tn611 mutants}

\subsection{Introduction}

Identifying the cross-sensitivity of the drug hypersensitive mutants identified in Chapter Three, and the differential-sensitivity to analogues of the drug that they were first identified as being hypersensitive to, can be used to generate more information on the specific role of the genes harbouring Tn611 insertions. Cross-sensitivity testing of the hypersensitive mutants generates information on whether the drug sensitivity of the mutants identified in Chapter Three is drug specific or a result of a general multi-drug resistance mechanism (drug non-specific). Differential-sensitivity to analogues of the drug that the mutant strain is hypersensitive to, where the mutants strains are either more or less sensitive to the analogues than the parent drug, can be used to group mutants that appear to contain insertions in genes involved in similar aspects of the drug's mode of action or drug resistance mechanisms. This approach is similar to target based whole-cell drug screening, whereby target information is integrated into screening for anti-mycobacterial activity (loerger et al., 2013; Nisa et al., 2010). The information from these assays for cross- and differentialsensitivity can be used to generate a more complete description of the drug mode of action.

\subsubsection{Analogues of anti-tuberculosis drugs}

The design and synthesis of drug analogues makes it possible to improve upon the properties of the originally isolated compound or to examine the mode of action of the drug. In the case of anti-TB drugs, analogues have been developed in an effort to improve anti-mycobacterial activity, especially against drug resistant strains, or to improve pharmacokinetic properties. For example, rifapentine is an analogue of rifampicin that has an increased half-life in the plasma of patients, allowing less frequent dosing (Acocella et al., 1971; Benator et al., 2002; Keung et al., 1999). This improves the likelihood that patients will adhere to the drug regimen, reducing the probability of rifapentine resistance occurring in the patient. Isoniazid was an intermediate generated when synthesising analogues of nicotinic acid with improved mycobacterial activity (Pansy et al., 1952). Isoniazid was found to have much greater activity than the other analogues of nicotinic acid that retained activity against TB, including pyrazinamide and ethionamide. 
Analogues are also used when studying drug structure-activity relationships (SAR), whereby the differential activity of the various analogues can provide information on key parts of the drug that are required for activity. This type of study can be combined with analysis of hypersensitive mutant strains to identify analogues that are less active against the mutants than expected. Differential-sensitivity of the mutant strain to different drug analogues indicates that the mode of action of the analogue differs in some way to that of the original drug, or that the analogue is resistant to a specific detoxification mechanism that defends against the original drug. As drugs are pleiotropic their complete anti-mycobacterial activity is likely to stem from a combination of both the action against the primary target and action against lesser secondary targets. Close structural analogues are generally expected to retain the complete mode of action of the parent drug; however, the activity against the specific target that generates most of its anti-mycobacterial activity may be altered and a secondary target of the parent drug may then take over to generate most of its anti-mycobacterial activity. As such, analogues that preferentially work via these secondary targets can be used as tools to identify these secondary targets, generating information on the overall mode of action of the parent drug. As the primary goal of the research described in this thesis was to gain a better understanding of the mode of action of these drugs in M. tuberculosis, it is important that analogues that transposon mutants demonstrate hypersensitivity to are active in M. tuberculosis. Any analogues that lack activity in M. tuberculosis are likely to have a mode of action specific to $M$. smegmatis.

\subsubsection{Isoniazid, rifampicin, and bedaquiline hypersensitive mutants}

The hypersensitive $M$. smegmatis mutant strains studied were the isoniazid hypersensitive mutants Tn::nudC and Tn::MSMEI_6092; the rifampicin hypersensitive mutants Tn::arr and Tn::MSMEI_3093; and the bedaquiline hypersensitive mutants Tn::atpB and Tn::MSMEI_3189. NudC has recently been demonstrated to be able to hydrolyse the INH-NAD adduct responsible for the inhibition of InhA (Wang et al., 2011). MSMEI_6092 encodes a Fur like protein of unknown function that likely regulates some aspect of the oxidative stress response. Arr is a known rifampicin-inactivating enzyme that works by ribosylating rifampicin (Dabbs et al., 1995; Quan et al., 1999). MSMEI_3093 encodes the 
ribosomal large subunit pseudouridine synthase $D$, although rifampicin hypersensitivity in the Tn::MSMEI_3093 strain may be due to altered transcription of the adjacent gene IspA (Pathak, 2013). Subunit A of the ATP synthase, encoded by atpB, is a subunit of the $F_{0}$ domain of the ATP synthase that is inhibited by bedaquiline. MSMEI_3189 encodes a glutamyl aminopeptidase, although the genetic basis of bedaquiline hypersensitivity for Tn::MSMEI_3189 remains unclear. These mutants were selected for further characterisation as they demonstrated the largest increases in sensitivity to the drugs tested; therefore it was reasoned that any cross-sensitivities or differential-sensitivities would be easier to identify than with the other mutants that showed a small increase in sensitivity.

\subsubsection{Objectives of Chapter Five}

The goal of Chapter Five was to further characterise a selection of the drug hypersensitive mutants identified in Chapter Three. Specific focus was given to the drug hypersensitive mutants' cross-sensitivity to other drugs and their differential-sensitivity to analogues of the drug that they were initially identified as being hypersensitive to.

To achieve this goal the following specific aims were addressed:

- Determine cross-sensitivity of the isoniazid hypersensitive transposon mutants Tn::nudC and Tn::MSMEI_6092, the rifampicin hypersensitive mutants Tn::arr and Tn::MSMEI_3093, and the bedaquiline hypersensitive mutants Tn::atpB and Tn::MSMEI_3189

- Determine the differential-sensitivity of the rifampicin hypersensitive mutants Tn::arr and Tn::MSMEI_3093, and the MSMEI_3093 overexpression strain, to the rifampicin analogue rifapentine

- Determine the differential-sensitivity of $T n:: n u d C$, and nudC, $\operatorname{inh} A$, and katG overexpression strains, to isoniazid analogues active against $M$. smegmatis $\mathrm{mc}^{2} 155$

- Confirm activity in M. tuberculosis of analogues that M. smegmatis transposon mutant strains showed differential-sensitivity to

To examine specificity of drug hypersensitivity, for each mutant that was identified in the primary testing (Chapters Three and Four) as being hypersensitive to one of the three drugs 
considered (isoniazid, rifampicin, and bedaquiline), the cross-sensitivities were determined for the other two drugs. The overexpression strains created in Chapter Four were used in the differential-sensitivity assay, with the exception of the katG overexpression strain which was generated in this chapter. Preliminary sensitivity testing of isoniazid analogues in M. tuberculosis was performed against the virulent strain $\mathrm{H}_{37} \mathrm{Rv}$.

\subsection{Methods}

\subsubsection{Cross sensitivity testing of transposon mutants to other drugs}

The cross-sensitivity was determined for the isoniazid hypersensitive mutants $T n:: n u d C$ and Tn::MSMEI_6092, the rifampicin hypersensitive mutants Tn::arr and Tn::MSMEI_3093, and the bedaquiline hypersensitive mutants Tn::atpB and Tn::MSMEI_3189 to rifampicin and bedaquiline, isoniazid and bedaquiline, and isoniazid and rifampicin, respectively. Cross-sensitivity of the hypersensitive strains was quantified by MIC using dose-response assays (section 2.8.3), and compared to the mean random transposon mutant control MIC. MICs were presented as the MIC ratio between the hypersensitive mutant and the mean control MIC.

\subsubsection{Differential-sensitivity testing of transposon mutants to drug analogues}

The differential-sensitivities of the rifampicin hypersensitive mutant strains Tn::arr and Tn::MSMEI_3093 to rifapentine, and the differential-sensitivities of the isoniazid hypersensitive mutant $T n:: n u d C$ to a range of isoniazid analogues were determined. Differential-sensitivity was quantified by MIC using dose-response assays (section 2.8.3). MIC values were given as the MIC ratio between the hypersensitive mutant and the MIC for the control strain D11. Differential-sensitivity was defined as a greater than 1.5 -fold difference in the MIC ratio of the analogue and the original drug (rifampicin or isoniazid). 


\subsubsection{Selection of isoniazid analogues for differential-sensitivity testing}

A total of 30 randomly selected isoniazid analogues were obtained from the National Institute of Health's (NIH) Developmental Therapeutics Program (DTP) repository, along with a sample of isoniazid. All analogues were dissolved in $100 \%$ dimethyl sulfoxide (DMSO) at a concentration of $10 \mathrm{mg} \cdot \mathrm{mL}^{-1}$ and stored at $-20{ }^{\circ} \mathrm{C}$. A full list of the isoniazid analogues, including their structural information is listed in appendix section 8.4. The isoniazid analogues were tested against WT $M$. smegmatis $\mathrm{mc}^{2} 155$ to determine their anti-mycobacterial activity (section 2.8.2), and only those analogues with sufficient activity were progressed into differential-susceptibility testing. Briefly, the analogues that showed greater than $75 \%$ growth-inhibition at a concentration of $100 \mu \mathrm{g} \cdot \mathrm{mL}^{-1}$ averaged (mean) across two separate experiments were taken to have sufficient activity for differential-sensitivity testing. The MICs of the isoniazid analogues that were active against M. smegmatis $\mathrm{mc}^{2} 155$ were determined by dose-response assay (section 2.8.3).

\subsubsection{Overexpression strains versus isoniazid analogues}

The MICs of the isoniazid analogues to which Tn::nudC showed differential-sensitivity were determined for the nudC and inhA overexpression strains constructed in Chapter Four, along with the empty vector control and random gene overexpression control strain. Overexpression assays were performed as described in section 2.8.3. Briefly, the MIC of the relevant analogue was determined by dose-response assay for the overexpression strains, both with and without vector promoter induction by $20 \mathrm{ng} \cdot \mathrm{mL}^{-1}$ tetracycline.

\subsubsection{1. katG overexpression strain versus isoniazid analogues}

M. smegmatis $\mathrm{mc}^{2} 155$ contains two homologues of katG, MSMEI_3380 and MSMEI_6216, with MSMEI_6216 sharing the greatest amino acid identity with the $M$. tuberculosis $\mathrm{H}_{37} \mathrm{Rv}$ katG gene, Rv1908c (appendix section 8.2). Therefore, for the purposes of this study MSMEI_6216 was used as the M. smegmatis $\mathrm{mc}^{2} 155$ katG gene (kat $G_{s m}$ ) for the overexpression assay and was amplified by PCR from WT M. smegmatis mc ${ }^{2} 155$ and cloned 
into the $B a m H I$ restriction site in plasmid pRC20 (section 2.6.9.1). The native $\mathrm{katG}_{s m}$ gene contains a BamHI site within its ORF, therefore overlap PCR was used to remove the restriction site by creating a silent mutation that was confirmed by sequencing (Table 2.9). Constructs of pRC20 containing $k a t G_{s m}$ were sequenced to check orientation and fidelity before transforming into WT $M$. smegmatis $\mathrm{mc}^{2} 155$ to generate the katG overexpression strain (section 2.6.2 and 2.6.7.2).

The $k a t G_{s m}$ overexpression assay was performed as described in section 2.8.3. Briefly, the MIC of the relevant analogue was determined by dose-response assay for the overexpression strain, both with and without vector promoter induction by $20 \mathrm{ng} \cdot \mathrm{mL}^{-1}$ tetracycline.

\subsubsection{M. tuberculosis $\mathrm{H}_{37} \mathrm{Rv}$ versus isoniazid analogues}

Preliminary determination of the sensitivity of $M$. tuberculosis $\mathrm{H}_{37} \mathrm{Rv}$ to the isoniazid analogues that the $T n:: n u d C$ strain showed differential-sensitivity to was performed in the PC3 laboratory at University College Dublin (Dublin, Ireland). Sensitivity was quantified by MIC determination in a dose-response assay, with one experiment performed (section 2.8.3). Briefly, MICs were determined by eye as the lowest concentration 24 hours after the addition of $50 \mu \mathrm{L} 0.03 \%$ resazurin to each well of the assay plates. 


\subsection{Results}

\subsubsection{Cross-sensitivities of hypersensitive mutants}

The cross-sensitivities of the hypersensitive mutants are presented in Table 5.1. The rifampicin hypersensitive mutant Tn::MSMEI_3093 showed a minor increase in resistance to isoniazid, and the bedaquiline hypersensitive mutants Tn::atpB and Tn::MSMEI_3189 showed minor increases in resistance to rifampicin, with Tn::MSMEI_3189 also showing a minor increase in resistance to isoniazid. No mutants showed an increased sensitivity to any of the other drugs tested.

Table 5.1: Cross-sensitivity of hypersensitive strains.

\begin{tabular}{lccc} 
& \multicolumn{3}{c}{ MIC ratio } \\
\hline Strain & Isoniazid & Rifampicin & Bedaquiline \\
\hline Isoniazid & & & \\
Tn::nudC & - & 1.2 & 0.67 \\
Tn::MSMEI_6092 & - & 1 & 1 \\
Rifampicin & & & \\
Tn::arr & 1 & - & 1 \\
Tn::MSMEI_3093 & 0.5 & - & 1.2 \\
Bedaquiline & & & \\
Tn::atpB & 1 & 0.5 & - \\
Tn::MSMEI_3189 & 0.5 & 0.5 & - \\
\hline
\end{tabular}

MIC ratios for hypersensitive mutants compared to the mean MIC of the random transposon mutant control strains for isoniazid, rifampicin, and bedaquiline. An MIC ratio of less than one (red shading) indicates increased resistance, while a ratio of more than one (green shading) indicates increased sensitivity, and a ratio of one (yellow shading) indicates no change in sensitivity for the hypersensitive mutant compared to the control strain D11.

\subsubsection{Differential-sensitivity of hypersensitive mutants}

\subsubsection{Rifapentine versus rifampicin hypersensitive transposon mutants}

The differential-sensitivities of the two rifampicin hypersensitive strains Tn::arr and Tn::MSMEI_3093 to the rifampicin analogue rifapentine were determined (Table 5.2). Both of the rifampicin hypersensitive strains showed similar increases in sensitivity to rifapentine compared to the control strains as had been seen with the parent compound rifampicin. 
Table 5.2: Sensitivity of rifampicin hypersensitive mutants to rifapentine.

\begin{tabular}{ccc}
\hline \multirow{2}{*}{ Drug } & \multicolumn{2}{c}{ MIC ratio } \\
\cline { 2 - 3 } & Tn::arr & Tn::MSMEI_3093 \\
\hline Rifampicin & 7.2 & 4.1
\end{tabular}

Rifapentine $8 \quad 5$

MIC ratios for the rifampicin hypersensitive strains Tn::arr and Tn::MSMEI_3093 compared to the random transposon mutant control strain D11 for rifampicin and rifapentine. A larger MIC ratio indicates increased sensitivity of the hypersensitive mutant compared to the control strain D11.

\subsubsection{Isoniazid analogues tested in isoniazid hypersensitive transposon mutants}

A selection of isoniazid analogues obtained from the DTP repository were tested for activity against WT M. smegmatis $\mathrm{mc}^{2} 155$ at $100 \mu \mathrm{g} \cdot \mathrm{mL}^{-1}$. A total of 14 of the 30 isoniazid analogues tested demonstrated greater than $75 \%$ growth-inhibition against WT M. smegmatis $\mathrm{mc}^{2} 155$ and were selected for differential-sensitivity testing of the isoniazid hypersensitive mutant Tn::nudC (Figure 5.2). The NSC numbers of the analogues that showed growth-inhibitory activity greater than the $75 \%$ cut-off were $6855,23449,27607,33759,33760,33763,36086$, $37379,37800,40350,59320,141142,176757$, and 322078. Information on these 14 analogues can be found in appendix section 8.4, along with structural details. Interestingly, all 14 of these analogues with activity in M. smegmatis had the core structure of isoniazid, varying only in their side chains (Figure 5.1). The total 30 analogues originally tested had a range of structures, with only 19 of the analogues retaining the core structure of isoniazid. Therefore, some of the analogues found to be inactive against WT M. smegmatis $\mathrm{mc}^{2} 155$ also had the structure indicated below. 


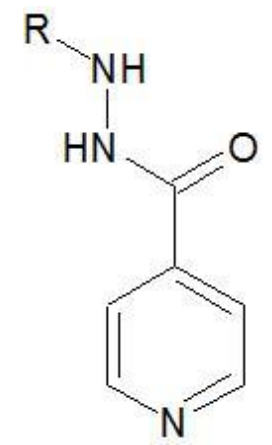

Figure 5.1: Structure of isoniazid analogues active against $M$. smegmatis $\mathrm{mc}^{2} 155$. Structure of analogues of isoniazid that showed greater than $75 \%$ growth-inhibition of WT M. smegmatis $\mathrm{mc}^{2} 155$ at $100 \mu \mathrm{g} \cdot \mathrm{mL}^{-1}$ where $\mathrm{R}$ represents the various side chains that differed among the 14 analogues.

Comparison of the activity against WT M. smegmatis mc $^{2} 155$ of all 19 analogues with the structure in Figure 5.1 revealed no patterns in terms of side chains of active and inactive isoniazid analogues (appendix section 8.4). 


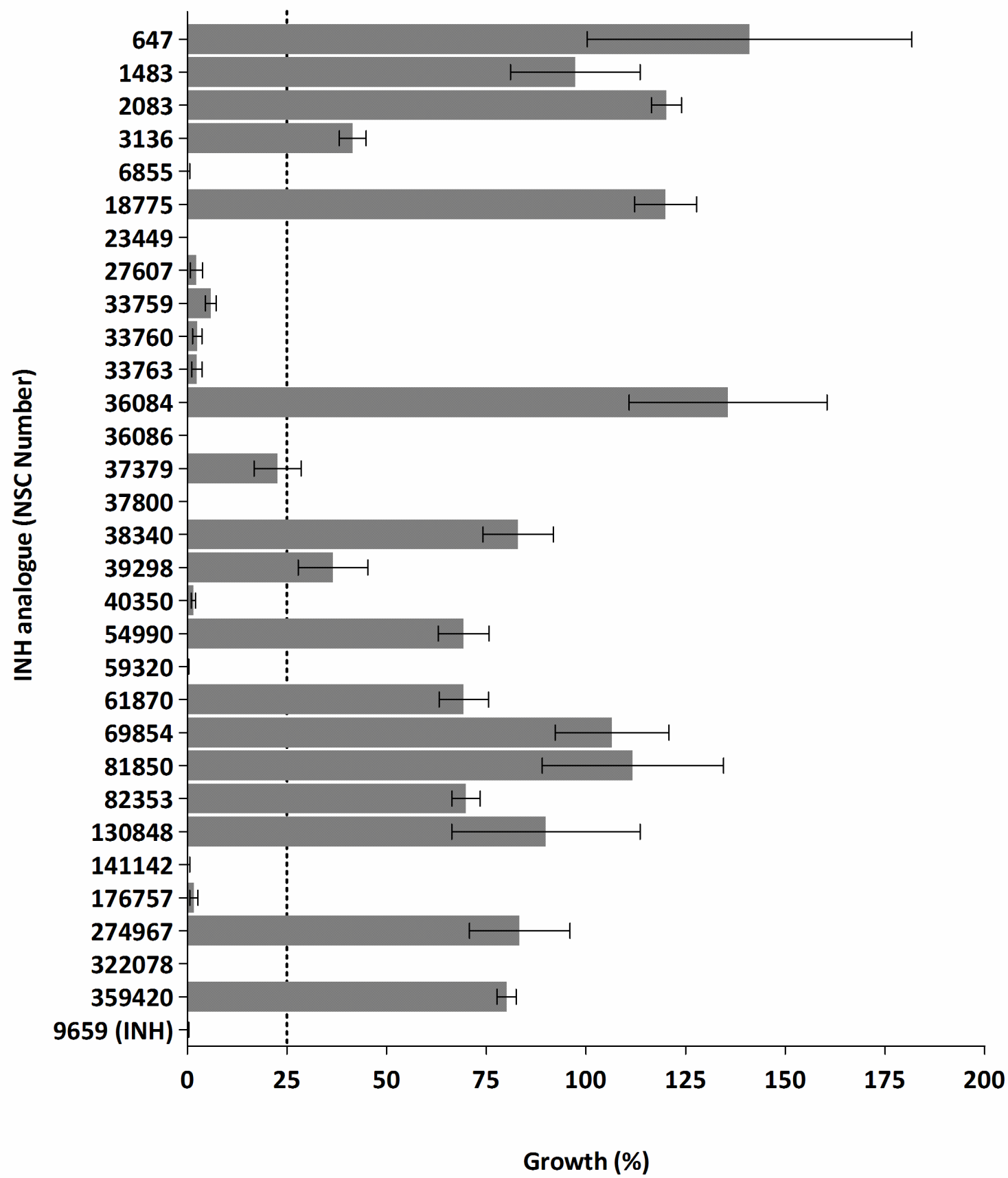

Figure 5.2: Growth-inhibition of WT $M$. smegmatis $\mathrm{mc}^{2} 155$ by isoniazid analogues. Columns represent mean growth of WT M. smegmatis $\mathrm{mc}^{2} 155$ in $100 \mu \mathrm{g} \cdot \mathrm{mL}^{-1}$ of each analogue across three independent experiments with standard error bars shown. Isoniazid analogues are named according to their NSC number. The dotted line at $25 \%$ growth indicates the activity cut-off of $75 \%$ growth-inhibition, with analogues that inhibited growth beyond this cut-off proceeding to the differential-sensitivity assay. 
The MICs of the 14 isoniazid analogues were determined for the isoniazid hypersensitive strain Tn::nudC and the random transposon control strain D11, with the MIC ratios for these two strains given in Table 5.3. The Tn::nudC strain showed an increased sensitivity compared to D11 for all 14 isoniazid analogues tested. The Tn::nudC strain was 4.8 -fold more sensitive to isoniazid compared to the control strain D11, and showed similar sensitivity to eight of the analogues. The Tn::nudC strain showed differential-sensitivity to the remaining six isoniazid analogues, showing only two - three-fold increases in sensitivity to these analogues compared to the control strain D11. The NSC numbers of the six analogues that $T n:: n u d C$ showed differential-sensitivity to were 27607, 33759, 37800, 40350, 141142, and 176757. There were no obvious patterns regarding the side chains of these analogues and their activity against $T n:: n u d C$.

Table 5.3: Sensitivity of Tn::nudC to isoniazid analogues.

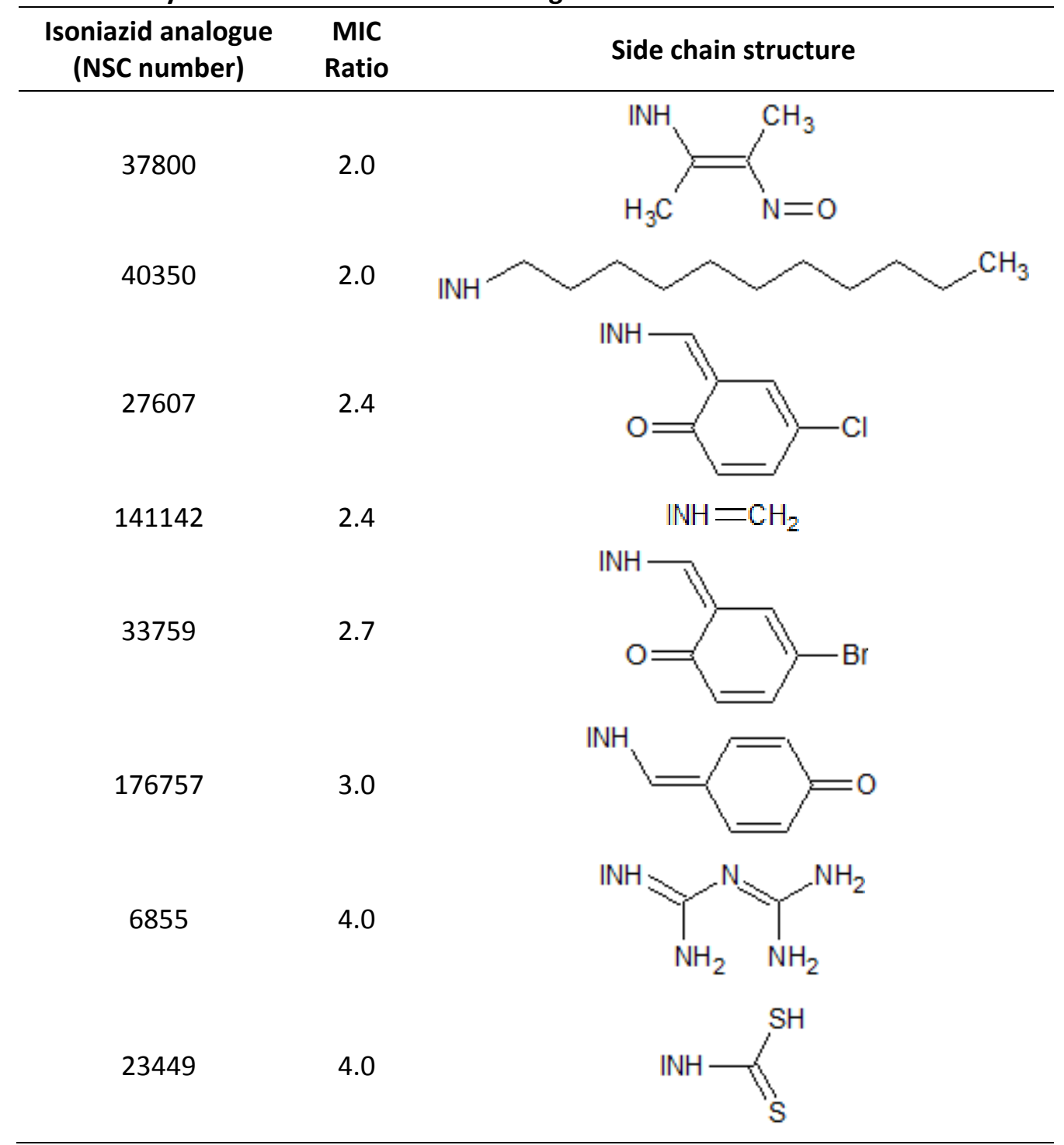




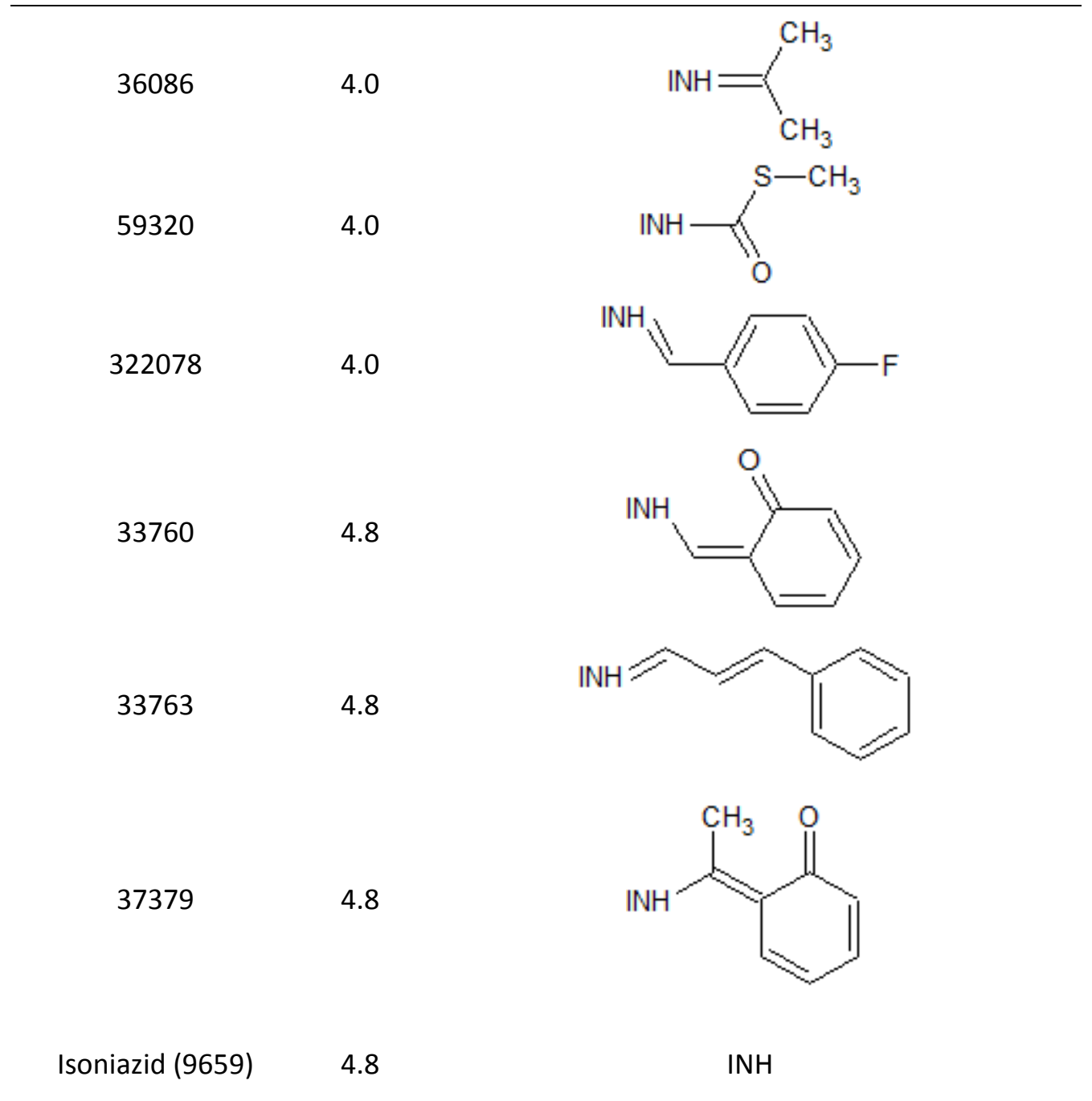

MIC ratios for the rifampicin hypersensitive strains Tn::arr and Tn::MSMEI_3093 compared to the control strain D11 for the 14 isoniazid analogues active against WT M. smegmatis $\mathrm{mc}^{2} 155$. A larger MIC ratio indicates increased sensitivity of the hypersensitive mutant compared to the control strain D11. Side chains from the core isoniazid (INH) structure are shown.

\subsubsection{Isoniazid analogues versus overexpression strains}

The sensitivities of the nudC and inhA overexpression strains to the six isoniazid analogues that Tn::nudC showed differential-sensitivity to were determined and compared to the random gene overexpression control strain and the empty vector control (Figure 5.3). Induction of expression from either the empty vector control or the random gene control MSMEI_4267 with $20 \mathrm{ng} \cdot \mathrm{mL}^{-1}$ tetracycline did not increase the MIC of any of the analogues greater than the basal two-fold increase in resistance observed for the empty vector control versus isoniazid (Figure 4.8). Induction of inhA expression resulted in a greater than 10-fold 
increase in resistance to analogues 37800,141142 , and 176757 and to isoniazid. Similarly, induction of nudC expression resulted in greater than 10-fold increases in resistance to analogues 37800,141142 , and 176757 and to isoniazid. No increase above the baseline was observed for the analogues, 27607 and 33759 when expression of either nudC or inhA was induced. No change in resistance was observed for analogue 40350 in any of the overexpression strains. 


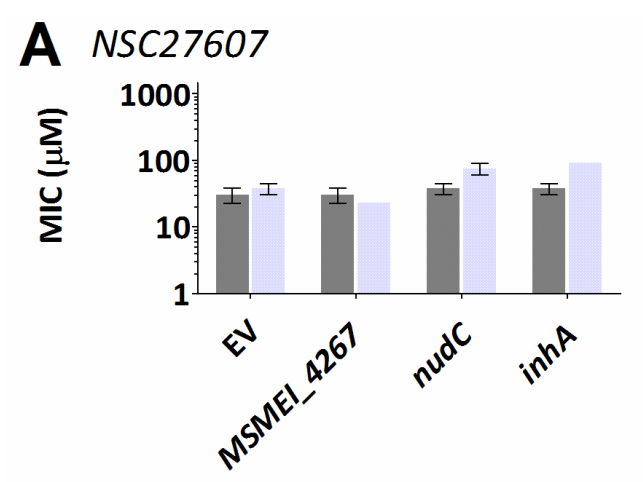

B NSC33759
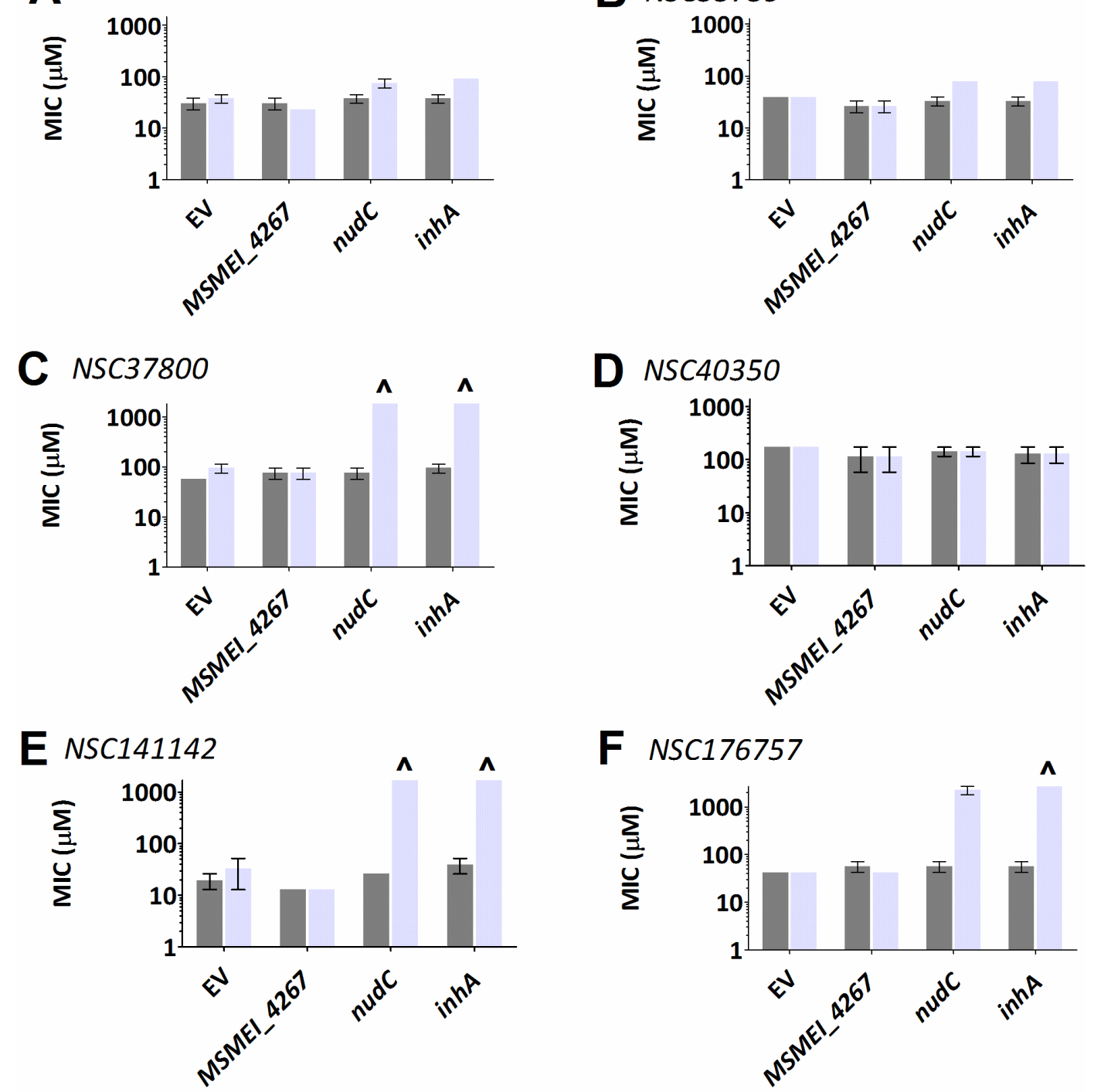

\section{G NSC9659 (isoniazid)}

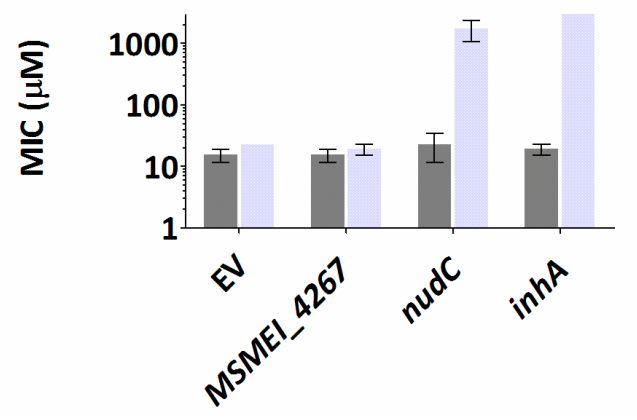

TC-

Tc+

Figure 5.3: Overexpression strains tested with isoniazid analogues. MICs are presented for the nudC, inhA, random gene MSMEI_4267 control, and empty vector (EV) control overexpression strains versus isoniazid analogues with and without promoter induction by $20 \mathrm{ng} \cdot \mathrm{mL}^{-1}$ tetracycline (Tc). The DTP repository number for each of the analogues is written in italics. Column heights represent mean MICs across three independent experiments with standard error bars shown. MICs greater than the highest concentrations tested were indicated with the caret symbol (^). 
The sensitivity of the $k_{a t G_{s m}}$ (MSMEI_6216) overexpression strain to isoniazid following induction with tetracycline was assessed (Figure 5.4). The $k a t G_{s m}$ over-expression strain was four-fold more sensitive to isoniazid following vector induction.

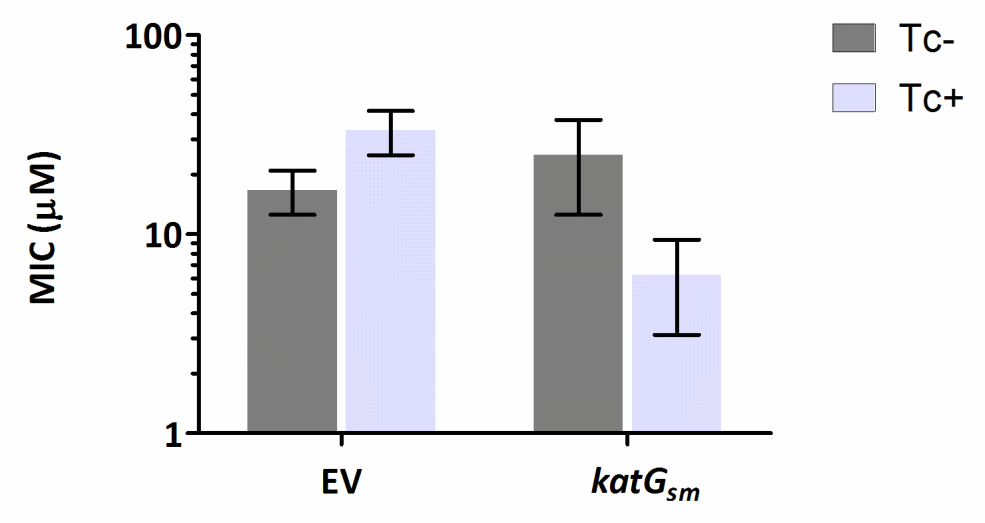

Strain

Figure 5.4: katG $_{s m}$ overexpression strain versus isoniazid. MIC of isoniazid for the empty vector (EV) control strain MRC20 and $k_{a t} G_{s m}$ overexpression strain, with and without promoter induction by $20 \mathrm{ng} \cdot \mathrm{mL}^{-1}$ tetracycline (Tc). Columns represent the mean MIC across three independent experiments with standard error bars shown.

The MICs of the six isoniazid analogues that Tn::nudC showed differential-sensitivity to were determined for the $k_{a t G_{s m}}$ overexpression strain (Figure 5.5). The $k a t G_{s m}$ overexpression strain showed increased sensitivity to all six isoniazid analogues following vector induction with tetracycline. The $k a t G_{s m}$ overexpression strain was five-fold more sensitive to the isoniazid analogue 40350, three-fold more sensitive to the analogues $27607,33759,141142$, and 176757 , and 2.5 -fold more sensitive to the analogue 37800 following vector promoter induction. 


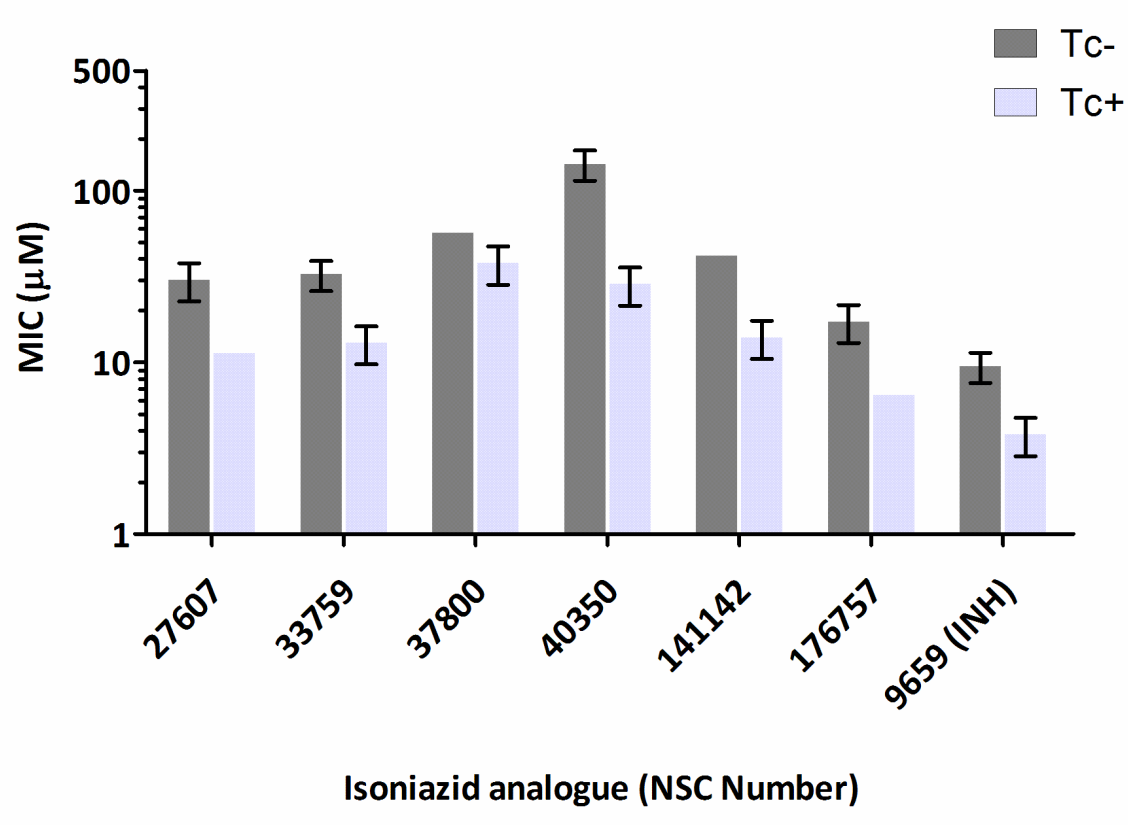

Figure 5.5: katG $_{s m}$ overexpression strain versus isoniazid analogues. MICs of isoniazid analogues and isoniazid (INH), for the $\mathrm{katG}_{s m}$ overexpression strain, with and without vector promoter induction with $20 \mathrm{ng} \cdot \mathrm{mL}^{-1}$ tetracycline (Tc). Columns represent mean MIC values across three independent experiments with standard error bars shown.

\subsubsection{Activity of isoniazid analogues in wild-type mycobacterial strains}

The six isoniazid analogues that showed differential activity against Tn::nudC (Table 5.3) were re-tested against WT $M$. smegmatis $\mathrm{mc}^{2} 155$ to determine their MICs. Preliminary testing was also carried out to determine the MIC of the isoniazid analogues for M. tuberculosis $\mathrm{H}_{37} \mathrm{Rv}$ (Table 5.4). For M. smegmatis $\mathrm{mc}^{2} 155$, analogues 37800 and 40350 had low activity compared to isoniazid, with MICs that were approximately three-fold and seven-fold higher than the MIC of isoniazid. The remaining analogues showed activity comparable to isoniazid, with MICs within two-fold of the MIC of isoniazid. For M. tuberculosis $\mathrm{H}_{37} \mathrm{Rv}$ only the two analogues 141142 and 37800 had activities comparable to isoniazid, with MICs approximately two-fold higher than the MIC of isoniazid. The remaining analogues had MICs for $M$. tuberculosis that were at least 15 -fold higher than that of isoniazid. 
Table 5.4: MICs of isoniazid analogues for WT M. smegmatis and M. tuberculosis.

\begin{tabular}{ccc} 
& MIC \pm se $(\boldsymbol{\mu M})$ & MIC $(\boldsymbol{\mu M})$ \\
\hline $\begin{array}{c}\text { INH Analogue } \\
\text { (NSC Number) }\end{array}$ & $\begin{array}{c}\text { M. smegmatis } \\
\text { mc }^{2} \mathbf{1 5 5}\end{array}$ & $\begin{array}{c}\text { M. tuberculosis } \\
\mathbf{H}_{37 \mathbf{R v}}\end{array}$ \\
\hline 27607 & $45.3 \pm 0.00$ & 363 \\
33759 & $58.7 \pm 19.5$ & 312 \\
37800 & $113 \pm 0.00$ & 28.4 \\
40350 & $257 \pm 85.8$ & 172 \\
141142 & $62.9 \pm 21.0$ & 21.0 \\
176757 & $25.9 \pm 0.00$ & 207 \\
9659 (isoniazid) & $34.2 \pm 11.4$ & 11.4 \\
\hline
\end{tabular}

MICs are presented for isoniazid and isoniazid analogues for WT M. smegmatis $\mathrm{mc}^{2} 155$ and M. tuberculosis $\mathrm{H}_{37} \mathrm{Rv}$. MICs for $M$. smegmatis represent mean MICs across three independent experiments with standard error (se) presented. MICs for $M$. tuberculosis $\mathrm{H}_{37} \mathrm{Rv}$ represent results from a single preliminary experiment.

\subsection{Discussion}

The first aim of this chapter was to characterise the cross-sensitivity of selected hypersensitive mutants identified in Chapter Three to other anti-mycobacterial drugs. The second aim was to investigate whether the hypersensitive mutants displayed differential-sensitivity to analogues of the drug that they were hypersensitive to. The sensitivities of relevant overexpression strains and of $M$. tuberculosis $\mathrm{H}_{37} \mathrm{Rv}$ were also determined for the isoniazid analogues that Tn::nudC demonstrated differential-sensitivity to, to further characterise their anti-mycobacterial activity.

\subsubsection{Cross-sensitivity of hypersensitive transposon mutants}

The two isoniazid, two rifampicin, and two bedaquiline hypersensitive mutants examined in this chapter were all tested for cross-sensitivity to the other two compounds of the three compounds used during the initial assay for drug hypersensitivity. None of the mutants examined showed cross-sensitivity to the other two drugs. This is in keeping with the fact that none of the mutant strains were identified in either the primary or secondary assays for drug hypersensitivity for more than one compound. These results indicate that the drug hypersensitivity of these six mutants is specific to the mode of action of these compounds, 
rather than a generic mechanism of drug resistance across two or more of the three drugs used (isoniazid, rifampicin, and bedaquiline). This statement is supported by the known roles of NudC and Arr in isoniazid and rifampicin sensitivity, respectively, whereby both enzymes are involved in detoxification of a specific drug (Alexander et al., 2003; Dabbs et al., 1995; Wang et al., 2011). Furthermore, these findings validate the screening process undertaken in this study as a highly specific method to identify mutants hypersensitive to a specific drug.

\subsubsection{Differential-sensitivities of hypersensitive transposon mutants to drug analogues}

Differential-sensitivity of the transposon mutants to analogues of the three parent drugs indicates that the analogues have modified modes of action compared to the parent drug, or that the analogues carry modifications that enable them to resist specific detoxification mechanisms. In this thesis the differential-sensitivity of transposon mutants to drug analogues was defined as a greater than 1.5 -fold difference in sensitivity to the analogue compared to the parent drug, where sensitivity was quantified in terms of the MIC ratio between the drug hypersensitive mutant and the random transposon control strain D11.

Investigation into differential-sensitivities of the rifampicin hypersensitive mutants Tn::arr and Tn::MSMEI_3093 revealed that these strains showed the same increase in sensitivity to rifapentine compared to the control strain D11 as they did to rifampicin. The finding that the relative sensitivities of the mutant strains were comparable for both drugs indicates that the genes containing the Tn611 insertion or adjacent to the insertion in these strains play similar roles in the mode of action or detoxification for rifampicin and rifapentine. Specifically, these results indicate that Arr is able to ribosylate and thereby detoxify rifapentine as well as the parent compound rifampicin. This was expected because of the structural similarity between rifapentine and rifampicin, and the fact that rifapentine is used clinically instead of rifampicin due to its increased half-life in the patient over rifampicin, rather than because it has altered activity or a different mode of action against mycobacteria (Benator et al., 2002; Keung et al., 1999).

The isoniazid hypersensitive strain $T n:: n u d C$ was also tested against a range of isoniazid analogues for differential-sensitivity. A total of 30 isoniazid analogues were randomly 
selected and obtained from the DTP repository, as well as a sample of isoniazid as a control, and tested against WT $M$. smegmatis $\mathrm{mc}^{2} 155$ to confirm their activity. Analogues that inhibited growth of WT $M$. smegmatis $\mathrm{mc}^{2} 155$ by at least $75 \%$, at a concentration of $100 \mu \mathrm{g} \cdot \mathrm{mL}^{-1}$, were chosen for further investigation. This initial experiment was performed to remove compounds with activities that were too low to be practical for determination of an MIC. Of the original 30 analogues, 14 analogues inhibited growth of WT M. smegmatis $\mathrm{mc}^{2} 155$ by at least $75 \%$, and interestingly all of these analogues shared the same basic structure, retaining the core isoniazid scaffold but with modification of a side chain, as depicted in Figure 5.1. Interestingly, of the 30 isoniazid analogues tested, 19 had the structure depicted in Figure 5.1. From examining the side chains of these 19 analogues, no common motifs distinguished the 14 analogues that had activity from the five analogues that did not. It is possible that the side chains of these analogues may facilitate or inhibit the interaction of the analogue with KatG, may alter drug permeability, or may act via a combination of these and other unknown factors.

The differential-sensitivity of the $T n:: n u d C$ strain to these 14 isoniazid analogues was tested by determining the MIC of these analogues for the Tn::nudC strain compared to the control strain D11. For isoniazid and eight of the analogues, Tn::nudC was approximately five-fold more sensitive than the D11 control strain. This suggests that these isoniazid analogues are acting in a similar manner to isoniazid, as had been predicted (section 5.1.1). Specifically, these results suggest that these analogues are interacting with NudC in the same way as isoniazid. Interestingly, $T n:: n u d C$ was only two - three-fold more sensitive than the control strain for six analogues $(27607,33759,37800,40350,141142$, and 176757). This suggests that the interaction between NudC and these six analogues is different to that of NudC and isoniazid. The finding that $T n:: n u d C$ was less sensitive than expected to these six analogues suggests that the protective role of NudC is less important for these six isoniazid analogues, and that its absence therefore has less of an effect on sensitivity. Interestingly, the isoniazid analogues that $T n:: n u d C$ showed differential-sensitivity to showed comparable activity to isoniazid (MICs within two-fold of the MIC of isoniazid), except for analogues 37800 and 40350, which were less active (three- and seven-fold increase in MIC compared to isoniazid). The role of NudC in resistance to the six analogues that Tn::nudC showed differential-sensitivity to was further investigated by overexpressing nudC in WT 
M. smegmatis $\mathrm{mc}^{2} 155$. These six analogues were also tested against the inhA overexpression strain to examine if they had a modified primary target as well as a loss of interaction with NudC.

Overexpression of nudC and inhA causes an increase in resistance to isoniazid by increasing the effective concentration of isoniazid needed to inhibit growth (Banerjee et al., 1994; Wang et al., 2011). In the present study it was found that overexpression of nudC or inhA had no effect on resistance to the isoniazid analogues 27607, 33759, and 40350. Combined with the lower than expected sensitivity of the Tn::nudC strain to these analogues, this finding suggests that NudC does not play a protective role for $M$. smegmatis $\mathrm{mc}^{2} 155$ against these particular analogues. One possibility is that the side chains of these analogues may cause steric hindrance, preventing NudC from hydrolysing any NAD adducts formed by these analogues. Alternatively, analogues 27607, 33759, and 40350 may inhibit the growth of mycobacteria by a mechanism independent of an NAD adduct. Indeed, the finding that overexpression of inhA does not increase resistance to these analogues suggests that InhA is not involved in the mode of action of these analogues. As mentioned previously, WT M. smegmatis $\mathrm{mc}^{2} 155$ was much less sensitive to analogue 40350 than isoniazid; however, analogues 27607 and 33759 showed activity in M. smegmatis similar to isoniazid which is to be expected if they are inhibiting the growth via a similar mode of action. Interestingly, the structures of analogues 27607 and 33759 have very similar side chains, differing only in the halogen atom (chlorine and bromine, respectively). It is possible that the side chains of these three analogues have anti-mycobacterial activity independent of the core isoniazid structure. An explanation for the InhA-independent activity of these three analogues is that they may inhibit the growth of $M$. smegmatis $\mathrm{mc}^{2} 155$ via secondary targets that are also part of the isoniazid mode of action. It would be of value to test the other isoniazid hypersensitive mutants identified in this thesis against isoniazid analogues 27607, 33759, 40350 to determine whether any of these strains also show differential-sensitivity. However, this was considered outside the scope of the immediate study.

The analogues 27607, 33759, and 40350 appeared to inhibit the growth of $M$. smegmatis $\mathrm{mc}^{2} 155$ in an NAD adduct independent manner. It has been demonstrated that the NudC of M. tuberculosis species cannot hydrolyse the INH-NAD adduct, and likely does not play a protective role in this species (Wang et al., 2011). However, the same study demonstrated 
that NudC from M. bovis BCG can hydrolyse the INH-NAD adduct and therefore may play a protective role in this species. Consequently, these analogues may have potential therapeutic applications in the treatment of $M$. bovis, which is responsible for a proportion of human TB disease, as well as disease of economically important livestock (Michel et al., 2010). Additionally, these analogues may have potential therapeutic value in the treatment of clinical isoniazid resistant inhA mutant or M. tuberculosis. Further testing of the activity of these analogues against $M$. bovis or isoniazid resistant $M$. tuberculosis was considered outside the scope of the current study.

Overexpression of nudC or inhA resulted in increased resistance to analogues 37800, 141142, and 176757, indicating that these particular analogues are likely to be working via an NAD adduct and thus inhibiting the growth of $M$. smegmatis $\mathrm{mc}^{2} 155$ in an InhA-dependant manner. The finding that the $\operatorname{Tn}:: n u d C$ strain showed less sensitivity to these analogues than expected can be explained by alternative detoxification mechanisms for these specific analogues.

\subsubsection{Role of KatG in activation of isoniazid analogues}

Isoniazid is a pro-drug that is activated by the catalase/peroxidase gene KatG. To test whether the isoniazid analogues were also activated by KatG, the sensitivity of a katG overexpression strain to these analogues was determined. Only the six isoniazid analogues that $T n:: n u d C$ showed differential-sensitivity to were tested against the $k a t G$ overexpression strain. M. smegmatis $\mathrm{mc}^{2} 155$ contains two copies of katG encoded by MSMEI_3380 and MSMEI_6216; whereas, M. tuberculosis $\mathrm{H}_{37} \mathrm{Rv}$ only contains one copy of katG, encoded by Rv1908c. Alignment of the protein sequences of MSMEI_3380 and MSMEI_6216 with Rv1908c revealed that MSMEI_6216 showed greater amino acid identity with Rv1908c. MSMEI_6216 was therefore selected as the katG overexpression strain for use in this study as its higher protein similarity to the $M$. tuberculosis gene suggested it would be most likely have the isoniazid activating activity. Had this assumption been incorrect and the MSMEI_6216 overexpression strain not shown increased sensitivity to isoniazid then a MSMEI_3380 overexpression strain would have been used instead, or a Rv1908c overexpression strain. The gene sequence for MSMEI_6216 contains a BamHI site, which 
was removed by introducing a silent mutation. The product of the modified MSMEI_6216 $\left(k a t G_{s m}\right)$ was able to activate isoniazid, as demonstrated by a four-fold increase in isoniazid sensitivity compared to the control strain. This result confirmed that the KatG enzyme encoded by MSMEI_6216 is capable of activating isoniazid and supported the initial selection of MSMEI_6216 for the katG overexpression assay.

With the exception of the analogue 40350, the katG overexpression strain showed comparable levels of increased sensitivity to all the isoniazid analogues tested, indicating KatG is able to activate all six analogues. However, the overexpression strain showed a much greater increase in sensitivity to 40350 than the other five analogues suggesting that the low activity of this analogue is primarily due to reduced activation by KatG, possibly due to steric hindrance of the long side chain. Interestingly, these results indicate that although the three analogues 27607,33759 , and 40350 appear to inhibit $M$. smegmatis $\mathrm{mc}^{2} 155$ independent of an NAD adduct, KatG is still able to activate these analogues. Although not pursued in this thesis, it would be valuable to know whether loss of function KatG mutants show decreased sensitivity to these analogues to confirm whether KatG is required for their activation. The apparent InhA- and NudC-independent mode of action of the three analogues 27607, 33759, and 40350 supports the idea that KatG-activated isoniazid reacts with more cellular components than just InhA (Argyrou et al., 2006; Mdluli et al., 1996, 1998; Rickman et al., 2013; Timmins and Deretic, 2006). These analogues may therefore be useful tools for elucidating what these other activated products are, and the identity of their targets.

\subsubsection{Activity of isoniazid analogues against $M$. tuberculosis}

Preliminary testing was performed to establish the sensitivity of $M$. tuberculosis $\mathrm{H}_{37} \mathrm{Rv}$ to the six isoniazid analogues that $T n:: n u d C$ showed differential-sensitivity to. The two analogues 37800 and 141142 showed activity similar to isoniazid, with MICs approximately two-fold greater than isoniazid. The remaining four analogues were inactive in $M$. tuberculosis $\mathrm{H}_{37} \mathrm{Rv}$, with MICs greater than 15-fold higher than that of isoniazid. The analogue 141142 had the most similar structure to isoniazid; however, there was no obvious connection between the structure of the side chain and the activity of the analogues. The reason for the low activity of the remaining five analogues in $M$. tuberculosis $\mathrm{H}_{37} \mathrm{Rv}$ is unclear. However, further testing 
of these analogues against $M$. tuberculosis is warranted to confirm the results from the single preliminary assay performed in this study.

\subsubsection{Summary}

None of the hypersensitive mutants tested showed cross-sensitivity to either of the other two drugs tested in this study. This indicates that the sensitivity of these mutants is specific to the original drug used in the screening process. Additionally, the two mutants identified in Chapter Three as being most hypersensitive to rifampicin showed no differential-sensitivity to the rifampicin analogue rifapentine. The isoniazid hypersensitive mutant $T n:: n u d C$ showed differential activity to six of the isoniazid analogues tested. Three of the six analogues likely work via an NAD adduct in an InhA-dependent manner similar to isoniazid. However, another detoxification mechanism in addition to NudC is suspected to exist for these analogues. The remaining three analogues $(27607,33759$, and 40350$)$ likely act in an InhA-independent manner that does not involve the formation of an NAD adduct. One possibility is that these analogues are inhibiting the growth of $M$. smegmatis $\mathrm{mc}^{2} 155$ by mechanisms specific to their side chains. Alternatively, these analogues may be acting via a secondary isoniazid mode of action. Interestingly the results presented here suggest that all of the six isoniazid analogues that demonstrated differential-activity against Tn::nudC can be activated by KatG, although it remains to be demonstrated if KatG is required for their activity. These results strongly support the concept that the mode of action of isoniazid is more complex than being mediated via inhibition of InhA alone. Furthermore, this study has identified three isoniazid analogues with apparent novel modes of action $(27607,33759$, and 40350). These three analogues will be useful tools in elucidating the nature of the secondary targets of isoniazid, and may have therapeutic use in the treatment of $M$. bovis and isoniazid resistant inhA mutant strains of $M$. tuberculosis. 


\section{General Discussion}

TB is the leading cause of morbidity and mortality by a bacterial pathogen, and there is a great need for the discovery of novel drugs that can shorten the treatment regimen, and effectively treat drug resistant cases, especially MDR-TB and XDR-TB (WHO, 2014). An important step in the drug discovery pathway is the identification of drug mode of action. Knowledge of drug mode of action enables rational modification of drugs to improve efficacy against both primary and secondary targets, and to decrease unwanted interactions or harmful side effects (Balganesh et al., 2008; loerger et al., 2013). To date, most studies investigating the modes of action of TB drugs have revolved around study of resistance-inducing mutations and altered transcription upon drug treatment, while little work had been done investigating mutations that induce hypersensitivity. Therefore, the aim of this thesis was to develop a high-throughput assay for identifying drug hypersensitive mutants of $M$. smegmatis $\mathrm{mc}^{2} 155$ in order to study the genetic basis of the drug hypersensitivity and generate information on drug mode action.

The two front-line anti-TB drugs isoniazid and rifampicin, along with bedaquiline, which is currently in phase III clinical trials, were selected for further investigation into their modes of action in this study. These drugs were selected because their primary targets are known and therefore the identification of mutations in genes already known to be involved in the drug mode or action or resistance mechanisms would validate our high-throughput screening assay for hypersensitivity mutations. Isoniazid is a prodrug that inhibits InhA, inhibiting mycolic acid biosynthesis (Banerjee et al., 1994). Isoniazid is activated by the catalase/peroxidase enzyme KatG, and reacts with NAD to form the INH-NAD adduct which binds to and inhibits InhA (Johnsson and Schultz, 1994; Rozwarski et al., 1998; Wilming and Johnsson, 1999; Zhang et al., 1992). Rifampicin inhibits transcription by binding to and inhibiting the $\beta$ subunit of RNA polymerase (RpoB) (Levin and Hatfull, 1993; Maggi et al., 1966; Wehrli et al., 1968). Bedaquiline inhibits the production of ATP by the ATP synthase, by binding to the $C$ subunit of the $F_{0}$ domain of the ATP synthase (Andries et al., 2005; Koul et al., 2007).

To the best of my knowledge, one study utilising an assay to screen for mutations that induce hypersensitivity to rifampicin has been published, albeit using a different endpoint, 
but no such studies have been published for isoniazid or bedaquiline. Therefore, it was expected that the assay for drug hypersensitivity used in this research would be able to identify genes not previously linked to isoniazid, rifampicin, or bedaquiline sensitivity. These genes may represent novel aspects of the known modes of action of these drugs, or completely novel modes of action. Both types of information can be used to improve our understanding of the complete modes of action of these drugs.

\subsection{Research summary}

\subsubsection{Isoniazid}

The assay for hypersensitivity successfully identified mutants with significantly increased sensitivity to isoniazid. The sensitivities of the 12 most hypersensitive mutants were quantified by MIC and subsequently the Tn611 insertion sites were determined for these mutants. Three mutants were much more sensitive to isoniazid compared to the controls than the other hypersensitive mutant strains, with two of these mutants containing a Tn611 insertion either within the nudC gene or in the adjacent gene MSMEI_1905. Complementation analysis suggested that the isoniazid hypersensitivity of both of these strains was due to a loss of function of nudC, whose protein product, NudC, is a protein recently identified in the detoxification of isoniazid. NudC hydrolyses the INH-NAD adduct that is the active form of isoniazid (Wang et al., 2011). As expected, given the role of NudC in detoxifying the INH-NAD adduct, the Tn::nudC mutant did not show any cross-sensitivity to either rifampicin or bedaquiline. Therefore these results strongly support a role for NudC in basal isoniazid resistance, as opposed to general multi-drug resistance. The isoniazid hypersensitive Tn::nudC mutant from the current study was identified prior to the publication of the results by Wang et al. (2011) and provides independent confirmation of the proposed role of NudC in basal isoniazid resistance in M. smegmatis $\mathrm{mc}^{2} 155$ (Wang et al., 2011).

The third isoniazid hypersensitive mutant that showed a large decrease in the MIC of isoniazid compared to the control had a Tn611 insertion within a gene (MSMEI_6092) likely to be involved in the oxidative stress response. This strain showed no cross-sensitivity to either rifampicin or bedaquiline, suggesting the mechanism of drug sensitisation is isoniazid 
specific. Complementation of this strain was not performed due to toxicity of the complementation vector during plasmid subcloning in E. coli, and therefore it is not possible to say definitively whether loss of functional MSMEI_6092 is responsible for the isoniazid hypersensitivity of Tn::MSMEI_6092. Interestingly, three other mutants with links to the oxidative stress response were identified in the assay for isoniazid hypersensitivity, although these mutants were not as sensitive as MSMEI_6092. These mutants had insertions within or adjacent to the $a h p C D$ locus, and two genetic loci involved in sulfur metabolism, a process closely linked to the oxidative stress response (Dussurget and Smith, 1998). It has previously been demonstrated that mutations that retard the oxidative stress response lead to increased isoniazid sensitivity due to a compensatory increase in the expression of other oxidative stress response genes, including $k a t G$, resulting in increased activation of isoniazid (Sherman et al., 1996). It seems likely that this mechanism is responsible for the isoniazid hypersensitivity of mutants identified in this thesis with Tn611 insertions within or adjacent to genes linked to the oxidative stress response.

Interestingly, only one isoniazid hypersensitive mutant that had a mutation in a gene with links to the mycolic acid biosynthesis pathway, which isoniazid inhibits, was identified. While it is possible that the transposon mutant collection generated in this thesis only contained this one mutant with an insertion in a mycolic acid biosynthesis genetic pathway, sequencing the insertion site of more mutants may have identified more hypersensitive mutants with insertions in genes related to mycolic acid biosynthesis. Strains with mutations in the mycolic acid biosynthesis pathway may have been less sensitive to isoniazid than the 12 mutants selected for further characterisation. Future investigations into the insertion sites of the other isoniazid hypersensitive mutants will reveal whether this was the case. The remaining sequenced isoniazid hypersensitive mutants had no clear links to the drug's known mode of action, or known resistance mechanisms. These genes therefore represent novel aspects of the mode of action or resistance mechanisms of isoniazid, and further study into the nature of their interaction with isoniazid will improve our understanding of the complete isoniazid mode of action. 


\subsubsection{Identification of isoniazid analogues with novel modes of action}

The differential-sensitivity of the Tn::nudC mutant was determined for 14 isoniazid analogues active against $M$. smegmatis $m^{2} 155$. All 14 analogues contained the core structure of isoniazid (Figure 5.1); the analogues that did not retain the core structure were found to be inactive against M. smegmatis. This indicates that these 14 analogues probably also retain the core mode of action of isoniazid, implicating the core structural component as an important part of the pharmacophore of the drug. The Tn::nudC mutant strain showed differential-sensitivity to six of the 14 isoniazid analogues, where the $T n:: n u d C$ strain was less sensitive to these analogues than expected. These six analogues were subsequently tested against an inhA and a nudC overexpression strain to investigate whether overexpression of these genes would lead to resistance to the analogues as it did for isoniazid. InhA is the primary target of isoniazid and its overexpression leads to isoniazid resistance. Three of these six analogues $(37800,141142$, and 176757) appeared to still inhibit the growth of $M$. smegmatis $\mathrm{mc}^{2} 155$ in an InhA-dependent manner despite the structural differences, as overexpression of InhA led to increased resistance to these three analogues. Additionally, these analogues appeared to inhibit growth via an INH-NAD adduct, as overexpression of NudC was able to increase resistance to these three analogues. These results, combined with the differential-sensitivity of Tn::nudC to these three analogues, suggests that while NudC can detoxify these analogues, it is not the only mechanism of detoxification as the Tn::nudC mutant showed little to no increase in sensitivity to these analogues.

The remaining three analogues $(27607,33759$, and 40350$)$ inhibited the growth of M. smegmatis $\mathrm{mc}^{2} 155$ in an InhA-independent manner as overexpression of InhA did not increase the resistance of $M$. smegmatis $\mathrm{mc}^{2} 155$ to these analogues. Additionally, NudC appeared unable to detoxify these analogues as overexpression of nudC did not increase the resistance of M.smegmatis $\mathrm{mc}^{2} 155$ to these analogues. Combined with the differential-sensitivity of $T n:: n u d C$ to these analogues, these results suggest that these analogues are not inhibiting the growth of M. smegmatis $\mathrm{mc}^{2} 155$ by inhibition of $\operatorname{Inh} A$, and not working via the INH-NAD adduct. 
A katG overexpression strain had increased sensitivity to all six analogues suggesting that KatG was able to metabolically activate all six isoniazid analogues. Combined with the previous observations, these results suggest that the three analogues 27607,33759 , and 40350, which were inhibiting the growth of $M$. smegmatis $\mathrm{mc}^{2} 155$ in an InhA-independent manner, were being activated by KatG to form growth-inhibitory metabolites other than the well characterised INH-NAD adduct. However, the Tn::nudC mutant still showed a slight increase in sensitivity to these analogues, suggesting at least some INH-NAD adduct may be being formed. Further studies of the growth inhibitory activity of these isoniazid analogues that are acting in an InhA-independent manner are warranted to determine their novel mechanisms of action, and to see where that mechanism of action overlaps with that of the parent compound.

Preliminary testing of the sensitivity of $M$. tuberculosis $\mathrm{H}_{37} \mathrm{Rv}$ was performed for the six analogues that Tn::nudC showed differential-sensitivity to, and the MIC values compared to their activities in $M$. smegmatis $\mathrm{mc}^{2} 155$. There were large differences in the relative activities of the analogues between the two species, including the three analogues that appeared to inhibit $M$. smegmatis $\mathrm{mc}^{2} 155$ in an InhA-independent manner, with only the analogues 37800 and 141142 showing any activity in $M$. tuberculosis $\mathrm{H}_{37} \mathrm{Rv}$. These results suggest that the target of the three inhA-independent analogues $(27607,33759$, and 40350$)$ may not be present in $M$. tuberculosis. Interestingly, it has been previously demonstrated that there are differences in the effect of isoniazid on $M$. smegmatis and M. tuberculosis, suggesting that the mode of action of isoniazid differs slightly between these two species (Mdluli et al., 1996). The NudC enzyme of $M$. tuberculosis $\mathrm{H}_{37} \mathrm{Rv}$ lacks INH-NAD hydrolysing activity, and it is predicted that the NudC of a large number of clinical isolates may also lack this hydrolysing activity (Wang et al., 2011). Despite these differences between strains, isoniazid analogues 27607, 33759, and 40350 will be useful in investigating the aspects of isoniazid's mode of action outside of the inhibition of InhA. Additionally, these analogues may find use in the treatment of $M$. bovis, as the NudC enzyme of $M$. bovis BCG is able to hydrolyse the INH-NAD adduct, and may play a protective role in $M$. bovis against isoniazid (Wang et al., 2011). These analogues may also have potential therapeutic value in the treatment of isoniazid resistant inhA mutant strains of $M$. tuberculosis. 


\subsubsection{Rifampicin}

The assay for hypersensitivity successfully identified mutants with increased sensitivity to rifampicin. The Tn611 insertion sites were successfully determined for 11 of the 12 most sensitive mutants validated in the secondary assay, and the hypersensitivities of these 12 mutants were quantified by MIC. Of these 12 mutants none showed any links to the known mode of action of rifampicin, and only three mutants had links to known rifampicin resistance mechanisms: Arr, an enzyme involved in a known rifampicin inactivation mechanism, PonA2, an enzyme involved in cell wall stability whose absence results in increased sensitivity to a range of lipophilic drugs including rifampicin, and the lipoprotein signal peptidase LspA. Two mutants had Tn611 insertions within two different multi-copy transposase genes, but which copies contained the Tn611 insertions, and therefore the genetic basis for the rifampicin hypersensitivity of these two mutants remains unknown. The remaining seven rifampicin hypersensitive mutants sequenced had insertions in genes with no clear links to the known mode of action of rifampicin or any of its known resistance mechanisms. These genes therefore represent novel mechanisms, and further study into the nature of their interaction with rifampicin will improve our understanding of the complete rifampicin mode of action. Of particular interest were two genes functionally related to the ESX-1 locus identified as containing Tn611 insertions in rifampicin hypersensitive mutants. The ESX-1 locus has not previously been implicated in the rifampicin mode of action or detoxification mechanism. These results therefore suggest a novel role for the ESX-1 locus in the rifampicin mode of action or detoxification pathways.

Rifampicin inhibits the RNA polymerase $\beta$ subunit (RpoB), inhibiting RNA transcription. Interestingly, none of the rifampicin hypersensitive mutants that were sequenced had mutations in genes that were known to be involved in transcription. This may suggest that these genes are essential in $M$. smegmatis $\mathrm{mc}^{2} 155$, and therefore mutants of these genes would not have been present in the transposon mutant collection constructed in this thesis. Alternatively, sequencing of more of the rifampicin hypersensitive mutants may have revealed mutants with insertions in genes related to transcription.

The two mutants most sensitive to rifampicin identified in Chapter Three were Tn::arr and Tn::MSMSEI_3093, which were eight- and four-fold more sensitive than the control strain, 
respectively. Tn::MSMEI_3093 contained a Tn611 insertion in the pseudouridine synthase D gene, which modifies selected uridine residues of the rRNA. Tn::arr was not complemented in this thesis as its involvement in rifampicin resistance in M. smegmatis is already well characterised. Complementation was also not performed for Tn::MSMEI_3093 due to an inability to generate a control strain for complementation and therefore the exact mechanism for this strain's rifampicin hypersensitivity remains unclear. However, it is possible that the rifampicin sensitivity may be due to altered transcription of the adjacent lipoprotein signal peptidase gene IspA rather than a loss of function of MSMEI_3093. Overexpression of IspA in $M$. tuberculosis has previously been shown to increase resistance to both rifampicin and isoniazid (Pathak, 2013). Interestingly, the transposon mutant Tn::MSMEI_3093 did not show cross-sensitivity to isoniazid in this thesis, suggesting that altered transcription of IspA may not be responsible for the rifampicin hypersensitivity of this strain. Alternatively, a loss of LspA function may cause increased rifampicin sensitivity but not increased isoniazid sensitivity. The two strains, Tn::arr and Tn::MSMEI_3093, were also examined for differential-sensitivity to rifapentine, an analogue of rifampicin. Both strains showed equal increases in sensitivity to rifapentine as they did to rifampicin, indicating that the role of these genes in the mode of action or resistance of rifampicin is conserved in rifapentine. Specifically, these results indicate that Arr can inactivate rifapentine, as well as its parent compound, rifampicin.

\subsubsection{Bedaquiline}

The assay for hypersensitivity successfully identified mutants with increased sensitivity to bedaquiline. The Tn611 insertion sites were determined for eight of the 12 most sensitive mutants validated in the secondary assay, and the hypersensitivity of these 12 mutants were quantified by MIC. Of the eight mutants that the insertion site was identified for, one had a Tn611 insertion in $m m p L 5$, which encodes the transmembrane transporter MmpL5 which is known to be involved in bedaquiline detoxification (Andries et al., 2014; Hartkoorn et al., 2014). An additional validated mutant had an insertion in $\operatorname{atp} B$, a gene that encodes the $A$ subunit of the Fo ATP synthase domain. Although this mutant was not one of the top bedaquiline sensitive strains, it was included in this thesis because atpB is related to the 
known mode of action of bedaquiline. The work on bedaquiline was performed in collaboration with Professor Greg Cook's research group at the University of Otago. The Tn::atpB mutant was identified in the primary assay for drug hypersensitivity, and was not one of the 12 most sensitive mutants further characterised in this thesis. However, a number of mutant strains that were identified in the primary screen of this thesis were sent to Professor Cook, who sequenced them and identified the Tn611 insertion site in the Tn::atpB mutant. Once identified it was decided to include $\operatorname{Tn}:$ atpB with the 12 most sensitive mutants for further characterisation.

Neither the most bedaquiline hypersensitive mutant, which contained a Tn611 insertion in a glutamyl peptidase gene (MSMEI_3189), nor the Tn::atpB mutant showed cross-sensitivity to either isoniazid or rifampicin. This result for the $\operatorname{Tn}:: a t p B$ mutant was expected, as it is known that the ATP synthase is related to the specific target of bedaquiline. Although the mechanism of bedaquiline hypersensitivity of the Tn::MSMEI_3189 mutant is unclear, it appears to be specific to bedaquiline.

\subsection{Critical evaluation of methodology}

\subsubsection{Limitation of using $M$. smegmatis mc $^{2} 155$ as the genetic model}

M. smegmatis $\mathrm{mc}^{2} 155$ was chosen as a genetic model for investigating anti-TB drug modes of action, primarily due to the time benefits the species provides over the slow growing models $M$. bovis BCG and $M$. tuberculosis $\mathrm{H}_{37} \mathrm{Ra}$. The fast growth rate of $M$. smegmatis increased the rate that the initial transposon mutants could be generated and then subsequently screened for drug hypersensitivity. This time benefit was offset, however, by the increased genetic differences between $M$. tuberculosis and the slow growing models such as $M$. bovis BCG. Therefore, any information on the mode of action generated from a study using $M$. smegmatis ultimately needs to be confirmed in M. tuberculosis. It has previously been proposed that one of the benefits of using $M$. smegmatis as a model is that it has a similar drug resistance profile as MDR-TB (Chaturvedi et al., 2007). However, the underlying genetic bases for these similar resistance phenotypes are very different. M. smegmatis contains drug detoxification mechanisms that $M$. tuberculosis does not have. 
For example, both Arr and NudC, which were identified in this study, are mechanisms of drug detoxification present in M. smegmatis but not M. tuberculosis.

The aim of this study was not to identify specific genes involved in the drug resistance phenotype, but rather to identify pathways that were sensitised to the drugs due to the presence of transposon insertions in their genes. This was achieved for isoniazid whereby multiple genes were identified that related to the oxidative stress response, and for rifampicin whereby two genes related to the ESX-1 locus were identified. However, the bedaquiline hypersensitive mutants did not reveal any such pattern of genes in a particular pathway. As discussed below, this may be a limitation of the number of mutants sequenced or the coverage of the library. An additional factor, however, is that the genome of $M$. smegmatis is not completely annotated, with numerous hypersensitive mutants identified in this study containing insertions in conserved, but hypothetical genes. This lack of annotation confounds the results as these genes with unknown function may be involved in the pathways of interest. Without better gene annotations it is not possible to determine from the literature if this is the case. The genomes of both $M$. tuberculosis and $M$. bovis are much better annotated and had the transposon mutant collection been constructed in one of these two species it would likely have been much easier to determine the role of any specific gene product. This situation is expected to improve with time as more of the genome of M. smegmatis $\mathrm{mc}^{2} 155$ is annotated.

\subsubsection{Use of $\operatorname{Tn} 611$ for transposon mutagenesis}

One of the advantages of random transposon mutagenesis is that the nature of the mutation is consistently a loss of function (Guilhot et al., 1994). However, after determining the transposon insertion sites for the drug hypersensitive mutants identified in this study, it was apparent that Tn611 also exerts a significant polar effect on the surrounding genes. Specifically, the Tn611 insertion in MSMEI_1905 had a polar effect on the adjacent nudC gene. Additionally, a number of hypersensitive mutants were identified that had intergenic Tn611 insertions. Thus, mutants within the collection may have altered transcription of more than one genetic locus, with the potential for the hypersensitivity to be due to the combined effect, as opposed to due to loss of function of a single gene product. This altered 
transcription could be down-regulation of adjacent genes or up-regulation driven by transposon internal promoters (Berg et al., 1980; Ciampi et al., 1982). To the best of my knowledge, little or no work has been done investigating the nature of the Tn611 insertion and its potential polar effects. Because most of the hypersensitive mutants identified in this study were not complemented, it is difficult to say with any certainty what the specific genetic basis for drug hypersensitivity was for all mutants other than the complemented Tn::nudC mutants.

Another limitation with the Tn611 transposon is that to the best of my knowledge the sequence into which it inserts has not been characterised. Although this transposon has previously been reported to insert in a random manner, and appears to provide good genome coverage, no studies as to which genes it can insert into have been performed. Most studies utilising transposon mutants now use a phage based transposon (Himar1), both for mutagenesis of M. smegmatis and M. tuberculosis (Siegrist and Rubin, 2009). The sequence where this transposon can be inserted has been characterised and, at least for M. tuberculosis, the genes that this transposon can insert into have been determined (Griffin et al., 2011; Sassetti et al., 2001, 2003). To the best of my knowledge no such information exists for Tn611. The Tn611 transposon was used for this study as attempts to generate transposon mutants with another phage-based system were unsuccessful, and the plasmid based system of Tn611 mutagenesis was found to be much easier for generating mutants. However, in hindsight, utilisation of the phage-based transposon Himar1 would have been advantageous as it is much better characterised than Tn611.

\subsubsection{Transposon mutant collection coverage}

Transposon insertions inherently label the site of the genetic lesion responsible for the mutant phenotype, enabling rapid identification of the phenotype's genetic basis. For this reason, random transposon mutagenesis is routinely employed for generating collections of random mutants of mycobacteria (both $M$. smegmatis and M. tuberculosis) for phenotypic screening. Another characteristic of transposon mutagenesis is the largely consistent nature of the mutations generated, in which insertion within a gene results in a loss of function mutation (Guilhot et al., 1994). However, this is also a major limitation of the approach in 
that only genes that are non-essential for the conditions being used can sustain transposon insertions. Mutants with insertions in genes that are essential under normal growth conditions will not be present in a random transposon mutant collection; therefore, these essential genes cannot be queried in phenotypic screening. However, the advantage of being able to rapidly identify the site of the mutation makes transposon mutagenesis a valuable methodology. As mentioned above, gene essentiality may be the reason mutants were not identified in the isoniazid or rifampicin screens that had transposon insertions within or adjacent to genes involved in fatty acid biosynthesis and transcription, respectively. The calculations estimating mutant collection coverage (section 3.2.1) assumed that the proportion of genes essential in M. smegmatis $\mathrm{mc}^{2} 155$ was the same as that for M. tuberculosis. An alternate method would have been to assume that the number of essential genes was consistent between the two species. The former option was selected as it would likely result in an underestimation of the number of essential genes, and it was decided this was preferable to an overestimation. This likely resulted in a slightly larger collection that had a higher genome coverage (and therefore more redundancy), which was preferable to a smaller collection with a lower genome coverage. The number of essential genes in $M$. smegmatis $m^{2} 155$ may actually be smaller than that of $M$. tuberculosis since significant portions of the $M$. smegmatis $\mathrm{mc}^{2} 155$ genome are duplicated. At least one gene that is essential in $M$. tuberculosis, atpD, is apparently non-essential in $M$. smegmatis $\mathrm{mc}^{2} 155$ due to multiple gene copies (Tran and Cook, 2005). The gene atpB, which was found to play a role in bedaquiline hypersensitivity, is in the same genetic region as atpD and may also be duplicated in the genome of $M$. smegmatis $\mathrm{mc}^{2} 155$.

Another limitation of the transposon mutant collection is that collection's size is related to the genomic coverage. As the transposition is random, the distribution of the insertion sites follows a normal distribution. It was predicted that the collection of 7680 mutants created in this thesis, which has approximately 1.1-fold genome coverage, would contain at least one transposon mutant for $73.2 \%$ of the non-essential genes. The implication of this is that $26.8 \%$ of the non-essential $M$. smegmatis $\mathrm{mc}^{2} 155$ genes are predicted to be excluded from the transposon mutant collection. These missing genes potentially include those related to the mode of action or resistance mechanisms of the drugs screened in this study, and may partially be responsible for the absence of mutants with insertions in pathways related to 
the respective drugs modes of action. The size of the collection created in the current study was chosen for a relatively high level of coverage while keeping the collection small enough to allow high-throughput screening. Increasing the size of the collection, and therefore its coverage, would have reduced the impact the incompleteness of the mutant collection had on the results of the hypersensitivity assays, potentially increasing the number of mutants identified as hypersensitive to each drug.

\subsubsection{Quantifying mutant hypersensitivity}

Quantification of the drug sensitivity of mutants validated in the secondary assay revealed that these mutants showed approximately two-fold or less increases in MIC compared to the controls, with the exception of the three and two most sensitive mutants from the isoniazid and rifampicin assays, respectively. As the variation in MIC was relatively small between the majority of validated hypersensitive mutants and the control strains, only the most sensitive mutants from each drug's assay were selected for complementation. Selection of only the most sensitive mutants allowed for clearer results in the complementation experiments as it was easier to determine if complementation was able to partially or completely restore WT sensitivity. Similarly, only the most sensitive mutants were selected for cross-sensitivity and differential-sensitivity testing. MICs are commonly used as the quantitative metric of drug sensitivity in the literature when discussing the sensitivity or resistance of mycobacteria to drugs and therefore were also used in this study. It was found in this thesis, however, that using MICs was not a very sensitive metric, with many of the dose-response assays showing variation close to the dilution factor used during serial dilution of the drugs (two-fold). This may have been mitigated by using smaller dilutions resulting in an improvement of the doseresponse assay variation.

The recovery of mutants with only small changes in drug MIC may correlate with the essentiality of those genes. This is corroborated by the fact that no genes that are known to be directly involved in the known modes of action of isoniazid or rifampicin were identified. It may be the genes involved in the drug mode of action are essential, meaning that only mutations in genes that were less directly involved in drug mode of action were present in the collection. A strain containing a Tn611 insertion in atpB which is known to be involved in 
the mode of action of bedaquiline was probably only recovered because of its duplication in the genome. Interestingly, a previous study of rifampicin hypersensitive transposon mutants of M. smegmatis $\mathrm{mc}^{2} 155$ identified a Tn::arr mutant similar to the one isolated in the present study (Alexander et al., 2003). However, this previously described Tn::arr mutant showed a 16-fold increase in MIC, compared to the eight-fold increase in MIC observed in this study. The major difference between these two studies was the use of transposon Tn611 in the present study and the Himar1 transposon in the study by Alexander et al. (2003) both transposons were delivered via temperature-sensitive delivery vectors; however, the non-permissive temperature for the $\operatorname{Tn} 611$ delivery vector was $41{ }^{\circ} \mathrm{C}$ compared to $37{ }^{\circ} \mathrm{C}$ for the Himar1 delivery vector (Siegrist and Rubin, 2009). Therefore, the rifampicin sensitivity of the Himar1 Tn::arr mutant was assessed at $37^{\circ} \mathrm{C}$, while all assays of $\operatorname{Tn} 611$ mutants were performed at $41^{\circ} \mathrm{C}$ in our study. This higher temperature may also be responsible for the observed low level of sensitivity of the isolated drug hypersensitive mutants. This highlights another reason why the use of a Himar1-based transposon would have been advantageous over the use of $\operatorname{Tn} 611$.

\subsubsection{Transposon mutant controls}

In the primary assay for drug hypersensitivity, the sensitivity of the mutants was expressed relative to the median sensitivity of the transposon mutant libraries, as it was assumed that the majority of mutants would have no increased sensitivity to the drug of interest. For the lower throughput secondary assay, the sensitivity of the mutants was validated, with the mutants ordered from most to least sensitive according to their growth-inhibition. Before further phenotypic and genotypic characterisation of the mutants, the hypersensitivities of the mutant strains were quantified using the MIC of the relevant drug. The selected control scheme was to use 10 mutant strains randomly selected from the mutant libraries, with the MICs of isoniazid, rifampicin, and bedaquiline averaged over the 10 strains. While WT M. smegmatis $\mathrm{mc}^{2} 155$ or WT M. smegmatis $\mathrm{mc}^{2} 155$ carrying a multi-copy plasmid with a kanamycin resistance gene were considered for the controls for this experiment, these strains would likely have had differing sensitivities to kanamycin compared with the transposon mutant strains. It is possible that the presence of kanamycin was altering the 
absolute sensitivity of the transposon mutants to the antibiotics, and therefore ideally the control should have the same level of kanamycin sensitivity as the transposon mutants. This condition was met by utilising random transposon mutant strains as the controls. It was expected that the chance of any one of the random transposon mutants selected as controls being hypersensitive or resistant to the drugs was low. Averaging the MICs for each drug across all 10 strains would compensate for any one strain that showed an altered sensitivity. When testing the differential-sensitivity of the strains against various analogues, only one of the 10 mutant strains was used due to the logistics of the assay and the large number of analogues screened. This strain, D11, showed a similar MIC for all three original drugs compared to the other 9 random control strains and therefore it was assumed that D11 would also show similar sensitivity to all the drug analogues. It would have been advantageous to have a WT control that had a single copy of extra-chromosomally encoded $a p h$, the kanamycin resistance gene, so that no assumptions needed to be made about the requirement of any of the genes in the random transposon mutant controls for drug sensitivity or resistance.

\subsection{Future directions}

The work in this thesis has identified many novel genes that confer hypersensitivity and are potentially involved in the known modes of action or detoxification pathways of isoniazid, rifampicin, and bedaquiline. In addition, potential novel modes of action or detoxification pathways were identified, with numerous future directions for this research.

- Completion of the sequencing and complementation of the isoniazid, rifampicin, and bedaquiline hypersensitive mutants identified in this study, as well as constructing knock-out mutants of genes containing Tn611 insertions. This would enable confirmation of the genetic basis for the hypersensitivity of these mutants. Better understanding of the genotypic basis would further improve our understanding of the complete modes of action of isoniazid, rifampicin, and bedaquiline facilitating rational drug design to improve drug treatment of TB.

- Expansion of the transposon mutant collection beyond 1.1-fold genome coverage, and screening the additional mutants against isoniazid, rifampicin, and bedaquiline 
would likely provide additional information on the mode of action of these drugs. Expansion of the library to three-fold coverage would mean that approximately $95 \%$ of the genes (versus $73.2 \%$ currently) predicted to be non-essential in $M$. smegmatis would be represented in the mutant collection. Further screening of isoniazid, rifampicin, and bedaquiline, would enable the identification of more novel genes in the modes of action or resistance mechanisms of these compounds, additional to those already identified in this thesis.

- The drug hypersensitive phenotype of many of the mutants identified in this study may not directly be due to a loss of function of the gene containing the transposon, but rather as a result of a perturbation in the genetic networks interacting with the drug target. These genetic networks include genes that are functionally related to the target such as those encoding other components of the targets functional pathway, regulators of the components of the targets functional pathway, as well as genes encoding enzymes that provide functional redundancy for components of the target pathway. Genetic interaction studies would reveal how the genes identified as containing transposon insertions in the drug hypersensitive mutants are interacting with the drug target or innate resistance mechanisms. Genetic interaction studies can be performed by studying synthetic lethality, where two viable single mutants are combined to produce a non-viable double mutant (Joshi et al., 2006; Ooi et al., 2006). This loss of viability indicates the two gene containing mutations have a genetic interaction. By generating collections of these double mutants a map of genetic interactions can be generated and used to identify functional relationships. Recent advances in the efficiency of generating targeted knock out mutations using allelic exchange in mycobacteria is making studies of genetic interactions more viable (Tufariello et al., 2014). This approach could be used to introduce secondary mutations into the transposon mutant collection in genes identified in this study as being involved in drug hypersensitivity to map their genetic interaction networks, and understand their functional relationship to the drug target or innate resistance mechanisms.

- This study has demonstrated the power of screening for hypersensitivity in identifying genes related to the modes of action and resistance mechanisms of anti-mycobacterial compounds. Therefore, future research using the assay developed 
in this thesis with novel anti-mycobacterial compounds would facilitate identification of their mode of action. Identification of the modes of action of novel antimycobacterials would enable the prioritisation of compounds with novel modes of action. Additionally, knowledge of the novel compounds modes of action would allow for rational modifications to improve their efficacy against TB. This would facilitate the discovery of new drugs with novel modes of action, and ultimately lead to an improvement in treatment of TB including MDR-TB.

- This study also identified three analogues of isoniazid that appear to inhibit the growth of M. smegmatis $\mathrm{mc}^{2} 155$ independent of the known target or isoniazid, InhA. Future work to identify the primary targets of these analogues may identify a novel drugable target in mycobacteria. Investigation into the cross-sensitivity of the target of these three analogues to isoniazid, will determine whether the primary target of these analogues is a secondary target for isoniazid. The identification of a secondary target for isoniazid would enable rational design of co-treatment options to improve the efficacy of isoniazid therapy of TB.

\subsection{Conclusions}

The aim of this thesis was to generate information on anti-TB drug mode of action by identifying the genetic basis of drug hypersensitivity in mutants of $M$. smegmatis $\mathrm{mc}^{2} 155$. The screening of a transposon mutant collection for isoniazid, rifampicin, and bedaquiline hypersensitive mutants confirmed a recently identified novel isoniazid resistance mechanism involving NudC detoxification, and highlighted a number of potentially new aspects of the modes of action of detoxification mechanisms for these drugs in M. smegmatis $\mathrm{mc}^{2} 155$. Additionally, this thesis demonstrated that analogues of current anti-TB drugs with modified modes of action with potential therapeutic use can be identified by integrating genetic information from a screen for hypersensitivity with chemical derivatisation. Specific outcomes from this thesis were:

- Novel genes that were related to the modes of action and resistance mechanisms of isoniazid, rifampicin, and bedaquiline were identified. This included genes related to the oxidative stress response that were identified in isoniazid hypersensitive 
mutants; and of particular interest, two genes (espR and MSMEI_5621) related to ESX-1 that were identified as containing Tn611 insertions in rifampicin hypersensitive mutants.

- NudC is responsible for detoxifying isoniazid, whereby a Tn::nudC mutant of M. smegmatis $\mathrm{mc}^{2} 155$ is hypersensitive to isoniazid and overexpression of NudC in M. smegmatis $\mathrm{mc}^{2} 155$ increases the MIC over 100 -fold. This independently verifies the results of Wang et al. (2011)

- The rifampicin ribosylating enzyme Arr, which can inactivate rifampicin, appears capable of also inactivating rifapentine, an analogue of rifampicin

- The isoniazid analogues NSC27607, NSC33759, and NSC40350 appear to inhibit the growth of $M$. smegmatis $\mathrm{mc}^{2} 155$ via a novel, InhA-independent, mode of action, with activity comparable to the parent drug in $M$. smegmatis $\mathrm{mc}^{2} 155$. These analogues may have potential therapeutic use in the treatment of infections by $M$. bovis or isoniazid resistant inhA mutant strains of $M$. tuberculosis

- Resazurin reduction is a valid surrogate for high-throughput screening of growth-inhibition of $M$. smegmatis $\mathrm{mc}^{2} 155$ when paired with a secondary $\mathrm{OD}_{600}$ based validation assay 


\section{References}

Abrahams, G.L., Kumar, A., Savvi, S., Hung, A.W., Wen, S., Abell, C., Barry III, C.E., Sherman, D.R., Boshoff, H.I.M., and Mizrahi, V. (2012). Pathway-selective sensitization of Mycobacterium tuberculosis for target-based whole-cell screening. Chem. Biol. 19, 844-854.

Acocella, G., Pagani, V., Marchetti, M., Baroni, G.C., and Nicolis, F.B. (1971). Kinetic studies on rifampicin. Chemotherapy 16, 356-370.

Adindla, S., Inampudi, K.K., Guruprasad, K., and Guruprasad, L. (2004). Identification and analysis of novel tandem repeats in the cell surface proteins of archaeal and bacterial genomes using computational tools. Comp. Funct. Genomics 5, 2-16.

Adler, J.J., and Rose, D.N. (1996). Transmission and pathogenesis of tuberculosis. Tuberculosis 1, 1002.

Alderton, H., and Smith, D. (2001). Safety in the laboratory. In Mycobacterium Tuberculosis Protocols, T. Parish, and N.G. Stoker, eds. (Humana Press), pp. 367-383.

Alexander, D.C., Jones, J.R.W., and Liu, J. (2003). A rifampin-hypersensitive mutant reveals differences between strains of Mycobacterium smegmatis and presence of a novel transposon, IS1623. Antimicrob. Agents Chemother. 47, 3208-3213.

Allen, B.W. (1998). Mycobacteria: General Culture Methodology and Safety Considerations. In Mycobacteria Protocols, (New Jersey: Humana Press), pp. 15-30.

Altaf, M., Miller, C.H., Bellows, D.S., and O'Toole, R. (2010). Evaluation of the Mycobacterium smegmatis and BCG models for the discovery of Mycobacterium tuberculosis inhibitors. Tuberculosis 90, 333-337.

Andries, K., Verhasselt, P., Guillemont, J., Göhlmann, H.W.H., Neefs, J.-M., Winkler, H., Gestel, J.V., Timmerman, P., Zhu, M., Lee, E., et al. (2005). A diarylquinoline drug active on the ATP synthase of Mycobacterium tuberculosis. Science 307, 223-227.

Andries, K., Villellas, C., Coeck, N., Thys, K., Gevers, T., Vranckx, L., Lounis, N., de Jong, B.C., and Koul, A. (2014). Acquired resistance of Mycobacterium tuberculosis to bedaquiline. PLoS ONE 9, e102135.

Anton, V., Rougé, P., and Daffé, M. (1996). Identification of the sugars involved in mycobacterial cell aggregation. FEMS Microbiol. Lett. 144, 167-170.

Argyrou, A., Vetting, M.W., Aladegbami, B., and Blanchard, J.S. (2006). Mycobacterium tuberculosis dihydrofolate reductase is a target for isoniazid. Nat. Struct. Mol. Biol. 13, 408413.

Azad, M.A., and Wright, G.D. (2012). Determining the mode of action of bioactive compounds. Bioorg. Med. Chem. 20, 1929-1939. 
Bacon, J., and Hatch, K.A. (2009). Continuous culture of mycobacteria. In Mycobacteria Protocols, T. Parish, and A.C. Brown, eds. (Humana Press), pp. 153-171.

Baker, M.A., Harries, A.D., Jeon, C.Y., Hart, J.E., Kapur, A., Lönnroth, K., Ottmani, S.-E., Goonesekera, S.D., and Murray, M.B. (2011). The impact of diabetes on tuberculosis treatment outcomes: A systematic review. BMC Med. 9, 81.

Balganesh, T.S., Alzari, P.M., and Cole, S.T. (2008). Rising standards for tuberculosis drug development. Trends Pharmacol. Sci. 29, 576-581.

Banerjee, A., Dubnau, E., Quemard, A., Balasubramanian, V., Um, K.S., Wilson, T., Collins, D., Lisle, G. de, and Jr., W.R.J. (1994). inhA, a gene encoding a target for isoniazid and ethionamide in Mycobacterium tuberculosis. Science 263, 227-230.

Bardarov, S., Kriakov, J., Carriere, C., Yu, S., Vaamonde, C., McAdam, R.A., Bloom, B.R., Hatfull, G.F., and Jacobs, W.R. (1997). Conditionally replicating mycobacteriophages: a system for transposon delivery to Mycobacterium tuberculosis. Proc. Natl. Acad. Sci. 94, 10961-10966.

Bassett, I.M., Lun, S., Bishai, W.R., Guo, H., Kirman, J.R., Altaf, M., and O'Toole, R.F. (2013). Detection of inhibitors of phenotypically drug-tolerant Mycobacterium tuberculosis using an in vitro bactericidal screen. J. Microbiol. 51, 651-658.

Belanger, A.E., Besra, G.S., Ford, M.E., Mikusová, K., Belisle, J.T., Brennan, P.J., and Inamine, J.M. (1996). The embAB genes of Mycobacterium avium encode an arabinosyl transferase involved in cell wall arabinan biosynthesis that is the target for the antimycobacterial drug ethambutol. Proc. Natl. Acad. Sci. U. S. A. 93, 11919-11924.

Benator, D., Bhattacharya, M., Bozeman, L., Burman, W., Cantazaro, A., Chaisson, R., Gordin, F., Horsburgh, C.R., Horton, J., Khan, A., et al. (2002). Rifapentine and isoniazid once a week versus rifampicin and isoniazid twice a week for treatment of drug-susceptible pulmonary tuberculosis in HIV-negative patients: a randomised clinical trial. Lancet 360, 528-534.

Bentrup, K.H. zu, and Russell, D.G. (2001). Mycobacterial persistence: adaptation to a changing environment. Trends Microbiol. 9, 597-605.

Berg, D.E., Weiss, A., and Crossland, L. (1980). Polarity of Tn5 insertion mutations in Escherichia coli. J. Bacteriol. 142, 439-446.

Bernstein, J., Lott, A., Steinberg, B., and Yale, H. (1952). Chemotherapy of experimental tuberculosis. Am Rev Tuberc 357-374.

Betts, J.C., Lukey, P.T., Robb, L.C., McAdam, R.A., and Duncan, K. (2002). Evaluation of a nutrient starvation model of Mycobacterium tuberculosis persistence by gene and protein expression profiling. Mol. Microbiol. 43, 717-731.

Billman-Jacobe, H., Sloan, J., and Coppel, R.L. (2006). Analysis of isoniazid-resistant transposon mutants of Mycobacterium smegmatis. FEMS Microbiol. Lett. 144, 47-52. 
Blokpoel, M.C.J., Murphy, H.N., O’Toole, R., Wiles, S., Runn, E.S.C., Stewart, G.R., Young, D.B., and Robertson, B.D. (2005). Tetracycline-inducible gene regulation in mycobacteria. Nucleic Acids Res. 33, e22.

Boshoff, H.I.M. (2004). The Transcriptional Responses of Mycobacterium tuberculosis to Inhibitors of Metabolism: Novel insights into drug mechanisms of action. J. Biol. Chem. 279, 40174-40184.

Bowman, J., and Ghosh, P. (2014). A complex regulatory network controlling intrinsic multidrug resistance in Mycobacterium smegmatis: Regulation of $M$. smegmatis drug resistance. Mol. Microbiol. 91, 121-134.

Brennan, P.J. (2003). Structure, function, and biogenesis of the cell wall of Mycobacterium tuberculosis. Tuberculosis 83, 91-97.

Brosch, R., Gordon, S.V., Garnier, T., Eiglmeier, K., Frigui, W., Valenti, P., Santos, S.D., Duthoy, S., Lacroix, C., Garcia-Pelayo, C., et al. (2007). Genome plasticity of BCG and impact on vaccine efficacy. Proc. Natl. Acad. Sci. 104, 5596-5601.

Brown, A.C., and Parish, T. (2006). Instability of the acetamide-inducible expression vector pJAM2 in Mycobacterium tuberculosis. Plasmid 55, 81-86.

Brown, C.A., Draper, P., and Hart, P.D. (1969). Mycobacteria and lysosomes: A paradox. Nature 221, 658-660.

Bucher, H.C., Griffith, L.E., Guyatt, G.H., Sudre, P., Naef, M., Sendi, P., and Battegay, M. (1999). Isoniazid prophylaxis for tuberculosis in HIV infection: a meta-analysis of randomized controlled trials. Aids 13, 501-507.

Bull, T.J., Hermon-Taylor, J., Pavlik, I., El-Zaatari, F., and Tizard, M. (2000). Characterization of IS900 loci in Mycobacterium avium subsp. paratuberculosis and development of multiplex PCR typing. Microbiology 146, 2185-2197.

Campbell, E.A., Korzheva, N., Mustaev, A., Murakami, K., Nair, S., Goldfarb, A., and Darst, S.A. (2001). Structural mechanism for rifampicin inhibition of bacterial RNA polymerase. Cell 104, 901-912.

Carroll, P., Muttucumaru, D.G.N., and Parish, T. (2005). Use of a tetracycline-inducible system for conditional expression in Mycobacterium tuberculosis and Mycobacterium smegmatis. Appl. Environ. Microbiol. 71, 3077-3084.

Carroll, P., Brown, A.C., Hartridge, A.R., and Parish, T. (2007). Expression of Mycobacterium tuberculosis Rv1991c using an arabinose-inducible promoter demonstrates its role as a toxin. FEMS Microbiol. Lett. 274, 73-82.

Caws, M., Thwaites, G., Dunstan, S., Hawn, T.R., Thi Ngoc Lan, N., Thuong, N.T.T., Stepniewska, K., Huyen, M.N.T., Bang, N.D., Huu Loc, T., et al. (2008). The influence of host and bacterial genotype on the development of disseminated disease with Mycobacterium tuberculosis. PLoS Pathog 4, e1000034. 
Chakraborty, S., Gruber, T., Barry, C.E., Boshoff, H.I., and Rhee, K.Y. (2013). Paraaminosalicylic acid acts as an alternative substrate of folate metabolism in Mycobacterium tuberculosis. Science 339, 88-91.

Chao, M.C., and Rubin, E.J. (2010). Letting sleeping dos lie: Does dormancy play a role in tuberculosis? Annu. Rev. Microbiol. 64, 293-311.

Chaturvedi, V., Dwivedi, N., Tripathi, R.P., and Sinha, S. (2007). Evaluation of Mycobacterium smegmatis as a possible surrogate screen for selecting molecules active against multi-drug resistant Mycobacterium tuberculosis. J. Gen. Appl. Microbiol. 53, 333-337.

Chen, L., Xie, Q., and Nathan, C. (1998). Alkyl hydroperoxide reductase subunit C (AhpC) protects bacterial and human cells against reactive nitrogen intermediates. Mol. Cell 1, 795805.

Chopra, I., and Brennan, P. (1998). Molecular action of anti-mycobacterial agents. Tuber. Lung Dis. 78, 89-98.

Ciampi, M.S., Schmid, M.B., and Roth, J.R. (1982). Transposon Tn10 Provides a Promoter for Transcription of Adjacent Sequences. Proc. Natl. Acad. Sci. U. S. A. 79, 5016-5020.

Clark-Curtiss, J.E., and Haydel, S.E. (2003). Molecular genetics of Mycobacterium tuberculosis pathogenesis. Annu. Rev. Microbiol. 57, 517-549.

Claxton, A.J., Cramer, J., and Pierce, C. (2001). A systematic review of the associations between dose regimens and medication compliance. Clin. Ther. 23, 1296-1310.

Click, E.S., Moonan, P.K., Winston, C.A., Cowan, L.S., and Oeltmann, J.E. (2012). Relationship between Mycobacterium tuberculosis phylogenetic lineage and clinical site of tuberculosis. Clin. Infect. Dis. 54, 211-219.

Cohen, S.P., Hächler, H., and Levy, S.B. (1993). Genetic and functional analysis of the multiple antibiotic resistance (mar) locus in Escherichia coli. J. Bacteriol. 175, 1484-1492.

Cole, S.T., Brosch, R., Parkhill, J., Garnier, T., Churcher, C., Harris, D., Gordon, S.V., Eiglmeier, K., Gas, S., Barry, C.E., et al. (1998). Deciphering the biology of Mycobacterium tuberculosis from the complete genome sequence. Nature 393, 537-544.

Dabbs, E.R., Yazawa, K., Mikami, Y., Miyaji, M., Morisaki, N., Iwasaki, S., and Furihata, K. (1995). Ribosylation by mycobacterial strains as a new mechanism of rifampin inactivation. Antimicrob. Agents Chemother. 39, 1007-1009.

Daugherty, A., Powers, K.M., Standley, M.S., Kim, C.S., and Purdy, G.E. (2011). Mycobacterium smegmatis RoxY is a repressor of oxyS and contributes to resistance to oxidative stress and bactericidal ubiquitin-derived peptides. J. Bacteriol. 193, 6824-6833.

Davis, A.L. (1996). History of the sanatorium movement. Tuberc. N. Y. Browand Co. 935-943. 
Deng, L., Mikusová, K., Robuck, K.G., Scherman, M., Brennan, P.J., and McNeil, M.R. (1995). Recognition of multiple effects of ethambutol on metabolism of mycobacterial cell envelope. Antimicrob. Agents Chemother. 39, 694-701.

Deshayes, C., Perrodou, E., Gallien, S., Euphrasie, D., Schaeffer, C., Van-Dorsselaer, A., Poch, O., Lecompte, O., and Reyrat, J.-M. (2007). Interrupted coding sequences in Mycobacterium smegmatis: authentic mutations or sequencing errors? Genome Biol. 8, R20.

Dhandayuthapani, S., Zhang, Y., Mudd, M.H., and Deretic, V. (1996). Oxidative stress response and its role in sensitivity to isoniazid in mycobacteria: characterization and inducibility of ahpC by peroxides in Mycobacterium smegmatis and lack of expression in $\mathrm{M}$. aurum and M. tuberculosis. J. Bacteriol. 178, 3641-3649.

Doerks, T., van Noort, V., Minguez, P., and Bork, P. (2012). Annotation of the M. tuberculosis hypothetical orfeome: Adding functional information to more than half of the uncharacterized proteins. PLOS ONE 7, e34302.

Dubos, R.J., and Dubos, J. (1952). The white plague: Tuberculosis, man, and society (Rutgers University Press).

Duncan, K. (2003). Progress in TB drug development and what is still needed. Tuberculosis 83, 201-207.

Dussurget, O., and Smith, I. (1998). Interdependence of mycobacterial iron regulation, oxidative-stress response and isoniazid resistance. Trends Microbiol. 6, 354-358.

Dye, C., and Williams, B.G. (2010). The population dynamics and control of tuberculosis. Science 328, 856-861.

Dye, C., Scheele, S., Dolin, P., Pathania, V., and Raviglione, M. (1999). Global burden of tuberculosis: Estimated incidence, prevalence, and mortality by country. JAMA 282, 677686.

Ehrt, S., Guo, X.Z.V., Hickey, C.M., Ryou, M., Monteleone, M., Riley, L.W., and Schnappinger, D. (2005). Controlling gene expression in mycobacteria with anhydrotetracycline and Tet repressor. Nucleic Acids Res. 33, e21.

Fernandes, P.B. (1988). Mode of action, and in vitro and in vivo activities of the fluoroquinolones. J. Clin. Pharmacol. 28, 156-168.

Ferraris, D.M., and Rizzi, M. (2011). Zinc-dependent metalloprotease-1 (Zmp1). In Encyclopedia of Inorganic and Bioinorganic Chemistry, (John Wiley \& Sons, Ltd),.

Ferraris, D.M., Sbardella, D., Petrera, A., Marini, S., Amstutz, B., Coletta, M., Sander, P., and Rizzi, M. (2011). Crystal structure of Mycobacterium tuberculosis zinc-dependent metalloprotease-1 (Zmp1), a metalloprotease involved in pathogenicity. J. Biol. Chem. 286, 32475-32482. 
Fleischmann, R.D., Alland, D., Eisen, J.A., Carpenter, L., White, O., Peterson, J., DeBoy, R., Dodson, R., Gwinn, M., Haft, D., et al. (2002). Whole-genome comparison of Mycobacterium tuberculosis clinical and laboratory strains. J. Bacteriol. 184, 5479-5490.

Gagneux, S., and Small, P.M. (2007). Global phylogeography of Mycobacterium tuberculosis and implications for tuberculosis product development. Lancet Infect. Dis. 7, 328-337.

Gagneux, S., DeRiemer, K., Van, T., Kato-Maeda, M., Jong, B.C. de, Narayanan, S., Nicol, M., Niemann, S., Kremer, K., Gutierrez, M.C., et al. (2006). Variable host-pathogen compatibility in Mycobacterium tuberculosis. Proc. Natl. Acad. Sci. U. S. A. 103, 2869-2873.

Galagan, J.E. (2014). Genomic insights into tuberculosis. Nat. Rev. Genet. 15, 307-320.

Galagan, J.E., Sisk, P., Stolte, C., Weiner, B., Koehrsen, M., Wymore, F., Reddy, T.B.K., Zucker, J.D., Engels, R., Gellesch, M., et al. (2010). TB database 2010: Overview and update. Tuberculosis 90, 225-235.

Galamba, A., Soetaert, K., Wang, X.-M., Bruyn, J.D., Jacobs, P., and Content, J. (2001). Disruption of adhC reveals a large duplication in the Mycobacterium smegmatis mc2155 genome. Microbiology 147, 3281-3294.

Gallien, S., Perrodou, E., Carapito, C., Deshayes, C., Reyrat, J.-M., Van Dorsselaer, A., Poch, O., Schaeffer, C., and Lecompte, O. (2009). Ortho-proteogenomics: multiple proteomes investigation through orthology and a new MS-based protocol. Genome Res. 19, 128-135.

Gannoun-Zaki, L., Alibaud, L., Carrère-Kremer, S., Kremer, L., and Blanc-Potard, A.-B. (2013). Overexpression of the KdpF membrane peptide in Mycobacterium bovis BCG results in reduced intramacrophage growth and altered cording morphology. PLoS ONE 8, e60379.

García-Fernández, E., Frank, D.J., Galán, B., Kells, P.M., Podust, L.M., García, J.L., and Ortiz de Montellano, P.R. (2013). A highly conserved mycobacterial cholesterol catabolic pathway. Environ. Microbiol. 15, 2342-2359.

Gill, W.P., Harik, N.S., Whiddon, M.R., Liao, R.P., Mittler, J.E., and Sherman, D.R. (2009). A replication clock for Mycobacterium tuberculosis. Nat. Med. 15, 211-214.

Ginsberg, A.M., and Spigelman, M. (2007). Challenges in tuberculosis drug research and development. Nature 13, 290-294.

Girardi, E., Raviglione, M.C., Antonucci, G., Godfrey-Faussett, P., and Ippolito, G. (1999). Impact of the HIV epidemic on the spread of other diseases: the case of tuberculosis. AIDS Lond. Engl. 14 Supp/ 3, S47-S56.

Goude, R., and Parish, T. (2009). Electroporation of mycobacteria. In Mycobacteria Protocols, T. Parish, and A.C. Brown, eds. (Humana Press), pp. 203-215.

Griffin, J.E., Gawronski, J.D., DeJesus, M.A., loerger, T.R., Akerley, B.J., and Sassetti, C.M. (2011). High-resolution phenotypic profiling defines genes essential for mycobacterial growth and cholesterol catabolism. PLoS Pathog 7, e1002251. 
Guilhot, C., Otal, I., Van Rompaey, I., Martin, C., and Gicquel, B. (1994). Efficient transposition in mycobacteria: construction of Mycobacterium smegmatis insertional mutant libraries. J. Bacteriol. 176, 535.

Gutacker, M.M., Smoot, J.C., Migliaccio, C.A.L., Ricklefs, S.M., Hua, S., Cousins, D.V., Graviss, E.A., Shashkina, E., Kreiswirth, B.N., and Musser, J.M. (2002). Genome-wide analysis of synonymous single nucleotide polymorphisms in Mycobacterium tuberculosis complex organisms: resolution of genetic relationships among closely related microbial strains. Genetics 162, 1533-1543.

Harisinghani, M.G., McLoud, T.C., Shepard, J.-A.O., Ko, J.P., Shroff, M.M., and Mueller, P.R. (2000). Tuberculosis from Head to Toe. RadioGraphics 20, 449-470.

Hartkoorn, R.C., Uplekar, S., and Cole, S.T. (2014). Cross-resistance between clofazimine and bedaquiline through upregulation of $\mathrm{MmpL} 5$ in Mycobacterium tuberculosis. Antimicrob. Agents Chemother. 58, 2979-2981.

Hopewell, P.C. (1992). Impact of human immunodeficiency virus infection on the epidemiology, clinical features, management, and control of tuberculosis. Clin. Infect. Dis. $15,540-547$.

Howard, S.T., and Byrd, T.F. (2000). The rapidly growing mycobacteria: saprophytes and parasites. Microbes Infect. 2, 1845-1853.

Huard, R.C., Fabre, M., Haas, P. de, Lazzarini, L.C.O., Soolingen, D. van, Cousins, D., and Ho, J.L. (2006). Novel genetic polymorphisms that further delineate the phylogeny of the Mycobacterium tuberculosis complex. J. Bacteriol. 188, 4271-4287.

loerger, T.R., O'Malley, T., Liao, R., Guinn, K.M., Hickey, M.J., Mohaideen, N., Murphy, K.C., Boshoff, H.I.M., Mizrahi, V., Rubin, E.J., et al. (2013). Identification of new drug targets and resistance mechanisms in Mycobacterium tuberculosis. PLoS ONE 8, e75245.

Ito, K., Yamamoto, K., and Kawanishi, S. (1992). Manganese-mediated oxidative damage of cellular and isolated DNA by isoniazid and related hydrazines: non-Fenton-type hydroxyl radical formation. Biochemistry (Mosc.) 31, 11606-11613.

Jarlier, V., and Nikaido, H. (1994). Mycobacterial cell wall: Structure and role in natural resistance to antibiotics. FEMS Microbiol. Lett. 123, 11-18.

Johnsson, K., and Schultz, P.G. (1994). Mechanistic studies of the oxidation of isoniazid by the catalase peroxidase from Mycobacterium tuberculosis. J. Am. Chem. Soc. 116, 74257426.

Joshi, S.A., Ball, D.A., Sun, M.G., Carlsson, F., Watkins, B.Y., Aggarwal, N., McCracken, J.M., Huynh, K.K., and Brown, E.J. (2012). EccA1, a component of the Mycobacterium marinum ESX-1 protein virulence factor secretion pathway, regulates mycolic acid lipid synthesis. Chem. Biol. 19, 372-380. 
Joshi, S.M., Pandey, A.K., Capite, N., Fortune, S.M., Rubin, E.J., and Sassetti, C.M. (2006). Characterization of mycobacterial virulence genes through genetic interaction mapping. Proc. Natl. Acad. Sci. 103, 11760-11765.

Kapopoulou, A., Lew, J.M., and Cole, S.T. (2011). The MycoBrowser portal: A comprehensive and manually annotated resource for mycobacterial genomes. Tuberculosis 91, 8-13.

Kaushal, D., Schroeder, B.G., Tyagi, S., Yoshimatsu, T., Scott, C., Ko, C., Carpenter, L., Mehrotra, J., Manabe, Y.C., Fleischmann, R.D., et al. (2002). Reduced immunopathology and mortality despite tissue persistence in a Mycobacterium tuberculosis mutant lacking alternative $\sigma$ factor, SigH. Proc. Natl. Acad. Sci. 99, 8330-8335.

Keung, A., Eller, M.G., McKenzie, K.A., and Weir, S.J. (1999). Single and multiple dose pharmacokinetics of rifapentine in man: Part II. Int. J. Tuberc. Lung Dis. 3, 437-444.

Kocagöz, T., Hackbarth, C.J., Unsal, I., Rosenberg, E.Y., Nikaido, H., and Chambers, H.F. (1996). Gyrase mutations in laboratory-selected, fluoroquinolone-resistant mutants of Mycobacterium tuberculosis H37Ra. Antimicrob. Agents Chemother. 40, 1768-1774.

Koul, A., Dendouga, N., Vergauwen, K., Molenberghs, B., Vranckx, L., Willebrords, R., Ristic, Z., Lill, H., Dorange, I., Guillemont, J., et al. (2007). Diarylquinolines target subunit c of mycobacterial ATP synthase. Nat. Chem. Biol. 3, 323-324.

Koul, A., Arnoult, E., Lounis, N., Guillemont, J., and Andries, K. (2011). The challenge of new drug discovery for tuberculosis. Nature 469, 483-490.

Krieger, I.V., Freundlich, J.S., Gawandi, V.B., Roberts, J.P., Gawandi, V.B., Sun, Q., Owen, J.L., Fraile, M.T., Huss, S.I., Lavandera, J.-L., et al. (2012). Structure-guided discovery of phenyldiketo acids as potent inhibitors of $M$. tuberculosis malate synthase. Chem. Biol. 19, 15561567.

Larsen, M.H., Vilchèze, C., Kremer, L., Besra, G.S., Parsons, L., Salfinger, M., Heifets, L., Hazbon, M.H., Alland, D., Sacchettini, J.C., et al. (2002). Overexpression of inhA, but not kasA, confers resistance to isoniazid and ethionamide in Mycobacterium smegmatis, $M$. bovis BCG and M. tuberculosis. Mol. Microbiol. 46, 453-466.

Lawn, S.D., and Zumla, A.I. (2011). Tuberculosis. The Lancet 378, 57-72.

Lechartier, B., Rybniker, J., Zumla, A., and Cole, S.T. (2014). Tuberculosis drug discovery in the post-post-genomic era. EMBO Mol. Med. 6, 158-168.

Levin, M.E., and Hatfull, G.F. (1993). Mycobacterium smegmatis RNA polymerase: DNA supercoiling, action of rifampicin and mechanism of rifampicin resistance. Mol. Microbiol. 8 , 277-285.

Lew, J.M., Kapopoulou, A., Jones, L.M., and Cole, S.T. (2011). TubercuList - 10 years after. Tuberculosis 91, 1-7. 
L'homme, R.F., Nijland, H.M.J., Gras, L., Aarnoutse, R.E., van Crevel, R., Boeree, M., Brinkman, K., Prins, J.M., Juttmann, J.R., and Burger, D.M. (2009). Clinical experience with the combined use of lopinavir/ritonavir and rifampicin. AIDS Lond. Engl. 23, 863-865.

Lienhardt, C., Glaziou, P., Uplekar, M., Lönnroth, K., Getahun, H., and Raviglione, M. (2012). Global tuberculosis control: lessons learnt and future prospects. Nat. Rev. Microbiol. 10, 407-416.

Lim, H., and Heffernan, H. (2013). Tuberculosis in New Zealand: Annual Report 2012 (Porirua: Institute of Environmental Science and Research Ltd (ESR)).

Lönnroth, K., Roglic, G., and Harries, A.D. (2014). Improving tuberculosis prevention and care through addressing the global diabetes epidemic: from evidence to policy and practice. Lancet Diabetes Endocrinol. 2, 730-739.

Maas, W.K. (1994). The arginine repressor of Escherichia coli. Microbiol. Rev. 58, 631-640.

Maggi, N., Pasqualucci, C.R., Ballotta, R., and Sensi, P. (1966). Rifampicin: A new orally active rifamycin. Chemotherapy 11, 285-292.

Manca, C., Lyashchenko, K., Colangeli, R., and Gennaro, M.L. (1997). MTC28, a novel 28kilodalton proline-rich secreted antigen specific for the Mycobacterium tuberculosis complex. Infect. Immun. 65, 4951-4957.

Manganelli, R., Voskuil, M.I., Schoolnik, G.K., Dubnau, E., Gomez, M., and Smith, I. (2002). Role of the extracytoplasmic-function $\sigma$ Factor $\sigma \mathrm{H}$ in Mycobacterium tuberculosis global gene expression. Mol. Microbiol. 45, 365-374.

Manjunatha, U., Boshoff, H.I.M., and Barry, C.E. (2009). The mechanism of action of PA-824. Commun. Integr. Biol. 2, 215-218.

Marais, B.J., Lönnroth, K., Lawn, S.D., Migliori, G. ni B., Mwaba, P., Glaziou, P., Bates, M., Colagiuri, R., Zijenah, L., Swaminathan, S., et al. (2013). Tuberculosis comorbidity with communicable and non-communicable diseases: integrating health services and control efforts. Lancet Infect. Dis. 13, 436-448.

Martin, C., Timm, J., Rauzier, J., Gomez-Lus, R., Davies, J., and Gicquel, B. (1990). Transposition of an antibiotic resistance element in mycobacteria. Nature 345, 739-743.

Martinez, A., Torello, S., and Kolter, R. (1999). Sliding motility in mycobacteria. J. Bacteriol. $181,7331-7338$.

Master, S.S., Davis, A.S., Rampini, S.K., Keller, C., Ehlers, S., Springer, B., Sander, P., and Deretic, V. (2008). Mycobacterium tuberculosis prevents inflammasome activation. Cell Host Microbe 3, 224-232.

Matsumoto, M., Hashizume, H., Tomishige, T., Kawasaki, M., Tsubouchi, H., Sasaki, H., Shimokawa, Y., and Komatsu, M. (2006). OPC-67683, a nitro-dihydro-imidazooxazole derivative with promising action against tuberculosis in vitro and in mice. PLoS Med 3, e466. 
Maus, C.E., Plikaytis, B.B., and Shinnick, T.M. (2005). Mutation of tlyA confers capreomycin resistance in Mycobacterium tuberculosis. Antimicrob. Agents Chemother. 49, 571-577.

McAdam, R.A., Quan, S., Smith, D.A., Bardarov, S., Betts, J.C., Cook, F.C., Hooker, E.U., Lewis, A.P., Woollard, P., Everett, M.J., et al. (2002). Characterization of a Mycobacterium tuberculosis H37Rv transposon library reveals insertions in 351 ORFs and mutants with altered virulence. Microbiology 148, 2975-2986.

McDermott, P.F., White, D.G., Podglajen, I., Alekshun, M.N., and Levy, S.B. (1998). Multidrug resistance following expression of the Escherichia coli marA gene in Mycobacterium smegmatis. J. Bacteriol. 180, 2995-2998.

Mdluli, K., Sherman, D.R., Hickey, M.J., Kreiswirth, B.N., Morris, S., Stover, C.K., and Barry, C.E. (1996). Biochemical and genetic data suggest that InhA is not the primary target for activated isoniazid in Mycobacterium tuberculosis. J. Infect. Dis. 174, 1085-1090.

Mdluli, K., Slayden, R.A., Zhu, Y., Ramaswamy, S., Pan, X., Mead, D., Crane, D.D., Musser, J.M., and Barry, C.E., 3rd (1998). Inhibition of a Mycobacterium tuberculosis beta-ketoacyl ACP synthase by isoniazid. Science 280, 1607-1610.

Mehta, P.K., King, C.H., White, E.H., Murtagh, J.J., and Quinn, F.D. (1996). Comparison of in vitro models for the study of Mycobacterium tuberculosis invasion and intracellular replication. Infect. Immun. 64, 2673-2679.

Michel, A.L., Müller, B., and van Helden, P.D. (2010). Mycobacterium bovis at the animalhuman interface: A problem, or not? Vet. Microbiol. 140, 371-381.

Miller, C.H., Nisa, S., Dempsey, S., Jack, C., and O'Toole, R. (2009). Modifying culture conditions in chemical library screening identifies alternative inhibitors of mycobacteria. Antimicrob. Agents Chemother. 53, 5279-5283.

Moazed, D., and Noller, H.F. (1987). Interaction of antibiotics with functional sites in $16 \mathrm{~S}$ ribosomal RNA. 327, 389-394.

Mostowy, S., Cleto, C., Sherman, D.R., and Behr, M.A. (2004). The Mycobacterium tuberculosis complex transcriptome of attenuation. Tuberculosis 84, 197-204.

Mudenda, V., Lucas, S., Shibemba, A., O’Grady, J., Bates, M., Kapata, N., Schwank, S., Mwaba, P., Atun, R., Hoelscher, M., et al. (2012). Tuberculosis and tuberculosis/HIV/AIDSassociated mortality in Africa: The urgent need to expand and invest in routine and research autopsies. J. Infect. Dis. jir859.

Murray, C.J., Styblo, K., and Rouillon, A. (1990). Tuberculosis in developing countries: burden, intervention and cost. Bull. Int. Union Tuberc. Lung Dis. 65, 6-24.

Mwandumba, H.C., Russell, D.G., Nyirenda, M.H., Anderson, J., White, S.A., Molyneux, M.E., and Squire, S.B. (2004). Mycobacterium tuberculosis resides in nonacidified vacuoles in endocytically competent alveolar macrophages from patients with tuberculosis and HIV infection. J. Immunol. 172, 4592-4598. 
Nair, S., Ramaswamy, P.A., Ghosh, S., Joshi, D.C., Pathak, N., Siddiqui, I., Sharma, P., Hasnain, S.E., Mande, S.C., and Mukhopadhyay, S. (2009). The PPE18 of Mycobacterium tuberculosis interacts with TLR2 and activates IL-10 induction in macrophage. J. Immunol. 183, 62696281.

Niemi, M., Backman, J.T., Fromm, M.F., Neuvonen, P.J., and Kivistö, K.T. (2003). Pharmacokinetic interactions with rifampicin: Clinical relevance. Clin. Pharmacokinet. 42, 819-850.

Nisa, S., Blokpoel, M.C.J., Robertson, B.D., Tyndall, J.D.A., Lun, S., Bishai, W.R., and O’Toole, R. (2010). Targeting the chromosome partitioning protein ParA in tuberculosis drug discovery. J. Antimicrob. Chemother. 65, 2347-2358.

Oatway Jr., W.H., and Steenken Jr., W. (1936). The pathogenesis and fate of tubercle produced by dissociated variants of tubercle bacilli. J. Infect. Dis. 59, 306-325.

O'Brien, R.J., and Nunn, P.P. (2001). The need for new drugs against tuberculosis. Am. J. Respir. Crit. Care Med. 163, 1055-1058.

Ooi, S.L., Pan, X., Peyser, B.D., Ye, P., Meluh, P.B., Yuan, D.S., Irizarry, R.A., Bader, J.S., Spencer, F.A., and Boeke, J.D. (2006). Global synthetic-lethality analysis and yeast functional profiling. Trends Genet. 22, 56-63.

Orme, I.M. (2014). A new unifying theory of the pathogenesis of tuberculosis. Tuberculosis 94, 8-14.

Palomino, J.C., and Portaels, F. (1999). Simple procedure for drug susceptibility testing of Mycobacterium tuberculosis using a commercial colorimetic assay. Eur. J. Clin. Microbiol. Infect. Dis. 18, 380-383.

Pandey, A.K., Raman, S., Proff, R., Joshi, S., Kang, C.M., Rubin, E.J., Husson, R.N., and Sassetti, C.M. (2009). Nitrile-inducible gene expression in mycobacteria. Tuberculosis 89, 12-16.

Pansy, F., Stander, H., and Donovick, R. (1952). In vitro studies on isonicotinic acid hydrazide. Am. Rev. Tuberc. 65, 761-764.

Parrish, N.M., Dick, J.D., and Bishai, W.R. (1998). Mechanisms of latency in Mycobacterium tuberculosis. Trends Microbiol. 6, 107-112.

Parsons, A.B., Brost, R.L., Ding, H., Li, Zhijiang, Zhang, Chaoying, Sheikh, B., Brown, Grant W., Kane, Patricia M., Hughes, Timothy R., and Boone, C. (2004). Integration of chemical genetic and genetic interaction data links bioactive compounds to cellular target pathways. Nat Biotech 22, 62-69.

Parsons, A.B., Lopez, A., Givoni, I.E., Williams, D.E., Gray, C.A., Porter, J., Chua, G., Sopko, R., Brost, R.L., Ho, C.-H., et al. (2006). Exploring the mode-of-action of bioactive compounds by chemical-genetic profiling in yeast. Cell 126, 611-625. 
Parwati, I., Alisjahbana, B., Apriani, L., Soetikno, R.D., Ottenhoff, T.H., Zanden, A.G.M. van der, Meer, J. van der, Soolingen, D. van, and Crevel, R. van (2010). Mycobacterium tuberculosis Beijing genotype is an independent risk factor for tuberculosis treatment failure in Indonesia. J. Infect. Dis. 201, 553-557.

Pathak, R. (2013). Role of Isp a gene in the biology and pathogenesis of mycobacterium tuberculosis. University of Delhi.

Patru, M.-M., and Pavelka, M.S. (2010). A role for the class A penicillin-binding protein PonA2 in the survival of Mycobacterium smegmatis under conditions of nonreplication. J. Bacteriol. 192, 3043-3054.

Payne, D.J., Gwynn, M.N., Holmes, D.J., and Pompliano, D.L. (2007). Drugs for bad bugs: confronting the challenges of antibacterial discovery. Nat. Rev. Drug Discov. 6, 29-40.

Perrodou, E., Deshayes, C., Muller, J., Schaeffer, C., Van Dorsselaer, A., Ripp, R., Poch, O., Reyrat, J.-M., and Lecompte, O. (2006). ICDS database: interrupted CoDing sequences in prokaryotic genomes. Nucleic Acids Res. 34, D338-D343.

Pethe, K., Sequeira, P.C., Agarwalla, S., Rhee, K., Kuhen, K., Phong, W.Y., Patel, V., Beer, D., Walker, J.R., Duraiswamy, J., et al. (2010). A chemical genetic screen in Mycobacterium tuberculosis identifies carbon-source-dependent growth inhibitors devoid of in vivo efficacy. Nat. Commun. 1, 57.

Pitulle, C., Dorsch, M., Kazda, J., Wolters, J., and Stackebrandt, E. (1992). Phylogeny of rapidly growing members of the genus Mycobacterium. Int. J. Syst. Bacteriol. 42, 337-343.

Prod'hom, G., Lagier, B., Pelicic, V., Hance, A.J., Gicquel, B., and Guilhot, C. (1998). A reliable amplification technique for the characterization of genomic DNA sequences flanking insertion sequences. FEMS Microbiol. Lett. 158, 75-81.

Prosser, G.A., and de Carvalho, L.P.S. (2013). Metabolomics reveal d-alanine:d-alanine ligase as the target of $d$-cycloserine in Mycobacterium tuberculosis. ACS Med. Chem. Lett.

Protopopova, M., Hanrahan, C., Nikonenko, B., Samala, R., Chen, P., Gearhart, J., Einck, L., and Nacy, C.A. (2005). Identification of a new antitubercular drug candidate, SQ109, from a combinatorial library of 1,2-ethylenediamines. J. Antimicrob. Chemother. 56, 968-974.

Pyle, M.M. (1947). Relative numbers of resistant tubercle bacilli in sputa of patients before and during treatment with streptomycin. Proc. Staff Meet. Mayo Clin. 22, 465-473.

Pym, A.S., Brodin, P., Brosch, R., Huerre, M., and Cole, S.T. (2002). Loss of RD1 contributed to the attenuation of the live tuberculosis vaccines Mycobacterium bovis BCG and Mycobacterium microti. Mol. Microbiol. 46, 709-717.

Pym, A.S., Brodin, P., Majlessi, L., Brosch, R., Demangel, C., Williams, A., Griffiths, K.E., Marchal, G., Leclerc, C., and Cole, S.T. (2003). Recombinant BCG exporting ESAT-6 confers enhanced protection against tuberculosis. Nat. Med. 9, 533-539. 
Quan, S., Imai, T., Mikami, Y., Yazawa, K., Dabbs, E.R., Morisaki, N., Iwasaki, S., Hashimoto, Y., and Furihata, K. (1999). ADP-Ribosylation as an Intermediate Step in Inactivation of Rifampin by a Mycobacterial Gene. Antimicrob. Agents Chemother. 43, 181-184.

Raghavan, S., Manzanillo, P., Chan, K., Dovey, C., and Cox, J.S. (2008). Secreted transcription factor controls Mycobacterium tuberculosis virulence. Nature 454, 717-721.

Ramaswamy, S., and Musser, J.M. (1998). Molecular genetic basis of antimicrobial agent resistance inMycobacterium tuberculosis: 1998 update. Tuber. Lung Dis. 79, 3-29.

Reed, M.B., Pichler, V.K., McIntosh, F., Mattia, A., Fallow, A., Masala, S., Domenech, P., Zwerling, A., Thibert, L., Menzies, D., et al. (2009). Major Mycobacterium tuberculosis Lineages Associate with Patient Country of Origin. J. Clin. Microbiol. 47, 1119-1128.

Rengarajan, J., Bloom, B.R., and Rubin, E.J. (2005). Genome-wide requirements for Mycobacterium tuberculosis adaptation and survival in macrophages. Proc. Natl. Acad. Sci. U. S. A. $102,8327-8332$.

Von Reyn, C.F., Kimambo, S., Mtei, L., Arbeit, R.D., Maro, I., Bakari, M., Matee, M., Lahey, T., Adams, L.V., Black, W., et al. (2011). Disseminated tuberculosis in human immunodeficiency virus infection: ineffective immunity, polyclonal disease and high mortality. Int. J. Tuberc. Lung Dis. 15, 1087-1092.

Rickman, K.A., Swancutt, K.L., Mezyk, S.P., and Kiddle, J.J. (2013). Isoniazid: Radical-induced oxidation and reduction chemistry. Bioorg. Med. Chem. Lett. 23, 3096-3100.

Riley, R. (1957). Aerial dissemination of pulmonary tuberculosis-the Burns Amberson Lecture. Am Rev Tuberc Pulm. Dis 76, 931-941.

Roback, P., Beard, J., Baumann, D., Gille, C., Henry, K., Krohn, S., Wiste, H., Voskuil, M.I., Rainville, C., and Rutherford, R. (2007). A predicted operon map for Mycobacterium tuberculosis. Nucleic Acids Res. 35, 5085-5095.

Rozwarski, D.A., Grant, G.A., Barton, D.H.R., Jacobs, W.R., and Sacchettini, J.C. (1998). Modification of the NADH of the Isoniazid Target (InhA) from Mycobacterium tuberculosis. Science 279, 98-102.

Sala, C., Haouz, A., Saul, F.A., Miras, I., Rosenkrands, I., Alzari, P.M., and Cole, S.T. (2009). Genome-wide regulon and crystal structure of Blal (Rv1846c) from Mycobacterium tuberculosis. Mol. Microbiol. 71, 1102-1116.

Sambrook, J., Russell, D.W., and Russell, D.W. (2001). Molecular cloning: a laboratory manual (3-volume set) (Cold spring harbor laboratory press Cold Spring Harbor, New York:).

Sassetti, C.M., Boyd, D.H., and Rubin, E.J. (2001). Comprehensive identification of conditionally essential genes in mycobacteria. Proc. Natl. Acad. Sci. 98, 12712-12717.

Sassetti, C.M., Boyd, D.H., and Rubin, E.J. (2003). Genes required for mycobacterial growth defined by high density mutagenesis. Mol. Microbiol. 48, 77-84. 
Saunders, B.M., and Cooper, A.M. (2000). Restraining mycobacteria: Role of granulomas in mycobacterial infections. Immunol. Cell Biol. 78, 334-341.

Saunders, N.J., Trivedi, U.H., Thomson, M.L., Doig, C., Laurenson, I.F., and Blaxter, M.L. (2011). Deep resequencing of serial sputum isolates of Mycobacterium tuberculosis during therapeutic failure due to poor compliance reveals stepwise mutation of key resistance genes on an otherwise stable genetic background. J. Infect. 62, 212-217.

Saviola, B., and Bishai, W. (2006). The Genus Mycobacterium--Medical. In The Prokaryotes, M.D.P. Dr, S. Falkow, E. Rosenberg, K.-H. Schleifer, and E. Stackebrandt, eds. (Springer New York), pp. 919-933.

Schnappinger, D., Ehrt, S., Voskuil, M.I., Liu, Y., Mangan, J.A., Monahan, I.M., Dolganov, G., Efron, B., Butcher, P.D., Nathan, C., et al. (2003). Transcriptional adaptation of Mycobacterium tuberculosis within macrophages insights into the phagosomal environment. J. Exp. Med. 198, 693-704.

Selwyn, P.A., Hartel, D., Lewis, V.A., Schoenbaum, E.E., Vermund, S.H., Klein, R.S., Walker, A.T., and Friedland, G.H. (1989). A prospective study of the risk of tuberculosis among intravenous drug users with human immunodeficiency virus infection. N. Engl. J. Med. 320, 545-550.

Shenoi, S., and Friedland, G. (2009). Extensively drug-resistant tuberculosis: a new face to an old pathogen. Annu. Rev. Med. 60, 307.

Sherman, D.R., Mdluli, K., Hickey, M.J., Arain, T.M., Morris, S.L., III, C.E.B., and Stover, C.K. (1996). Compensatory ahpC Gene Expression in Isoniazid-Resistant Mycobacterium tuberculosis. Science 272, 1641-1643.

Shi, W., Zhang, X., Jiang, X., Yuan, H., Lee, J.S., Barry, C.E., Wang, H., Zhang, W., and Zhang, Y. (2011). Pyrazinamide inhibits trans-translation in Mycobacterium tuberculosis. Science 333, 1630-1632.

Shiloh, M.U., and DiGiuseppe Champion, P.A. (2010). To catch a killer. What can mycobacterial models teach us about Mycobacterium tuberculosis pathogenesis? Curr. Opin. Microbiol. 13, 86-92.

Shinnick, T.M., and Good, R.C. (1994). Mycobacterial taxonomy. Eur. J. Clin. Microbiol. Infect. Dis. 13, 884-901.

Siegrist, M.S., and Rubin, E.J. (2009). Phage transposon mutagenesis. In Mycobacteria Protocols, T. Parish, and A.C. Brown, eds. (Humana Press), pp. 311-323.

Singh, R., Manjunatha, U., Boshoff, H.I.M., Ha, Y.H., Niyomrattanakit, P., Ledwidge, R., Dowd, C.S., Lee, I.Y., Kim, P., Zhang, L., et al. (2008). PA-824 kills nonreplicating Mycobacterium tuberculosis by intracellular NO release. Science 322, 1392-1395. 
Smith, N.H., Kremer, K., Inwald, J., Dale, J., Driscoll, J.R., Gordon, S.V., van Soolingen, D., Glyn Hewinson, R., and Maynard Smith, J. (2006). Ecotypes of the Mycobacterium tuberculosis complex. J. Theor. Biol. 239, 220-225.

Snapper, S.B., Melton, R.E., Mustafa, S., Kieser, T., and Jr, W.R.J. (1990). Isolation and characterization of efficient plasmid transformation mutants of Mycobacterium smegmatis. Mol. Microbiol. 4, 1911-1919.

Srivastava, V., Rouanet, C., Srivastava, R., Ramalingam, B., Locht, C., and Srivastava, B.S. (2007). Macrophage-specific Mycobacterium tuberculosis genes: identification by green fluorescent protein and kanamycin resistance selection. Microbiology 153, 659.

Steenken, W., Oatway, W.H., and Petroff, S.A. (1934). Biological studies of the tubercule bacillus. J. Exp. Med. 60, 515-540.

Stop TB Partnership (2006). The Global Plan to Stop TB 2006-2015: Actions for Life: Towards a World Free of Tuberculosis (Stop TB Partnership).

Stover, C.K., de la Cruz, V.F., Fuerst, T.R., Burlein, J.E., Benson, L.A., Bennett, L.T., Bansal, G.P., Young, J.F., Lee, M.H., Hatfull, G.F., et al. (1991). New use of BCG for recombinant vaccines. Nature 351, 456-460.

Stover, C.K., Warrener, P., VanDevanter, D.R., Sherman, D.R., Arain, T.M., Langhorne, M.H., Anderson, S.W., Towell, J.A., Yuan, Y., McMurray, D.N., et al. (2000). A small-molecule nitroimidazopyran drug candidate for the treatment of tuberculosis. Nature 405, 962-966.

Suthar, A.B., Lawn, S.D., del Amo, J., Getahun, H., Dye, C., Sculier, D., Sterling, T.R., Chaisson, R.E., Williams, B.G., Harries, A.D., et al. (2012). Antiretroviral therapy for prevention of tuberculosis in adults with HIV: A systematic review and meta-analysis. PLoS Med 9, e1001270.

Tahlan, K., Wilson, R., Kastrinsky, D.B., Arora, K., Nair, V., Fischer, E., Barnes, S.W., Walker, J.R., Alland, D., Barry, C.E., et al. (2012). SQ109 targets MmpL3, a membrane transporter of trehalose monomycolate involved in mycolic acid donation to the cell wall core of Mycobacterium tuberculosis. Antimicrob. Agents Chemother. 56, 1797-1809.

Tanaka, Y., Yazawa, K., Dabbs, E.R., Nishikawa, K., Komaki, H., Mikami, Y., Miyaji, M., Morisaki, N., and Iwasaki, S. (1996). Different rifampicin inactivation mechanisms in nocardia and related taxa. Microbiol. Immunol. 40, 1-4.

Timmins, G.S., and Deretic, V. (2006). Mechanisms of action of isoniazid. Mol. Microbiol. 62, 1220-1227.

Timmins, G.S., Master, S., Rusnak, F., and Deretic, V. (2004). Nitric oxide generated from isoniazid activation by KatG: Source of nitric oxide and activity against Mycobacterium tuberculosis. Antimicrob. Agents Chemother. 48, 3006-3009.

Timperio, A.M., Rinalducci, S., and Zolla, L. (2005). Hydrazide derivatives produce active oxygen species as hydrazine. Bioorganic Chem. 33, 459-469. 
Tran, S.L., and Cook, G.M. (2005). The F1Fo-ATP synthase of Mycobacterium smegmatis is essential for growth. J. Bacteriol. 187, 5023-5028.

Triccas, J.A., Parish, T., Britton, W.J., and Gicquel, B. (1998). An inducible expression system permitting the efficient purification of a recombinant antigen from Mycobacterium smegmatis. Fems Microbiol. Lett. 167, 151-156.

Tufariello, J.M., Malek, A.A., Vilchèze, C., Cole, L.E., Ratner, H.K., González, P.A., Jain, P., Hatfull, G.F., Larsen, M.H., and Jacobs, W.R. (2014). Enhanced Specialized Transduction Using Recombineering in Mycobacterium tuberculosis. mBio 5, e01179-14.

Udwadia, Z.F., Amale, R.A., Ajbani, K.K., and Rodrigues, C. (2012). Totally drug-resistant tuberculosis in India. Clin. Infect. Dis. 54, 579-581.

Uhía, I., Galán, B., Kendall, S.L., Stoker, N.G., and García, J.L. (2012). Cholesterol metabolism in Mycobacterium smegmatis. Environ. Microbiol. Rep. 4, 168-182.

Uplekar, M., Figueroa-Munoz, J., Floyd, K., Getahun, H., and Jaramillo, E. (2006). The Stop TB Strategy: building on and enhancing DOTS to meet the TB-related Millennium Development Goals.

Vandal, O.H., Roberts, J.A., Odaira, T., Schnappinger, D., Nathan, C.F., and Ehrt, S. (2009). Acid-Susceptible Mutants of Mycobacterium tuberculosis Share Hypersusceptibility to Cell Wall and Oxidative Stress and to the Host Environment. J. Bacteriol. 191, 625-631.

Villemagne, B., Crauste, C., Flipo, M., Baulard, A.R., Déprez, B., and Willand, N. (2012). Tuberculosis: The drug development pipeline at a glance. Eur. J. Med. Chem. 51, 1-16.

Wang, F., Jain, P., Gulten, G., Liu, Z., Feng, Y., Ganesula, K., Motiwala, A.S., loerger, T.R., Alland, D., Vilchèze, C., et al. (2010). Mycobacterium tuberculosis Dihydrofolate Reductase Is Not a Target Relevant to the Antitubercular Activity of Isoniazid. Antimicrob. Agents Chemother. 54, 3776-3782.

Wang, X.-D., Gu, J., Wang, T., Bi, L.-J., Zhang, Z.-P., Cui, Z.-Q., Wei, H.-P., Deng, J.-Y., and Zhang, X.-E. (2011). Comparative analysis of mycobacterial NADH pyrophosphatase isoforms reveals a novel mechanism for isoniazid and ethionamide inactivation. Mol. Microbiol. 82, 1375-1391.

Wang, X.-M., Galamba, A., Warner, D.F., Soetaert, K., Merkel, J.S., Kalai, M., Bifani, P., Lefèvre, P., Mizrahi, V., and Content, J. (2008). IS1096-mediated DNA rearrangements play a key role in genome evolution of Mycobacterium smegmatis. Tuberculosis 88, 399-409.

Wehrli, W., Knüsel, F., Schmid, K., and Staehelin, M. (1968). Interaction of rifamycin with bacterial RNA polymerase. Proc. Natl. Acad. Sci. 61, 667-673.

Weiling Hong, Wanyan Deng, and Jianping Xie (2013). The Structure, Function, and Regulation of Mycobacterium Ftsz. Cell Biochem. Biophys. 65, 97-105. 
Wengenack, N.L., and Rusnak, F. (2001). Evidence for Isoniazid-Dependent Free Radical Generation Catalyzed by Mycobacterium tuberculosis KatG and the Isoniazid-Resistant Mutant KatG(S315T). Biochemistry (Mosc.) 40, 8990-8996.

WHO (2010). The Global Plan to Stop TB 2011-2015: Transforming the fight towards the elimination of tuberculosis.

WHO (2014). Global tuberculosis report 2014.

Williams, K.J., Joyce, G., and Robertson, B.D. (2010). Improved mycobacterial tetracycline inducible vectors. Plasmid 64, 69-73.

Wilming, and Johnsson (1999). Spontaneous formation of the bioactive form of the tuberculosis drug isoniazid. Angew. Chem. Int. Ed Engl. 38, 2588-2590.

Yen, S., Bower, J.E., Freeman, J.T., Basu, I., and O'Toole, R.F. (2013). Phylogenetic lineages of tuberculosis isolates in New Zealand and their association with patient demographics. Int. J. Tuberc. Lung Dis. 17, 892-897.

Young, D.B., Perkins, M.D., Duncan, K., and Barry, C.E. (2008). Confronting the scientific obstacles to global control of tuberculosis. J. Clin. Invest. 118, 1255-1265.

Zhang, H., Gao, L., Zhang, J., Li, W., Yang, M., Zhang, H., Gao, C., and He, Z.-G. (2014). A Novel marRAB Operon Contributes to the Rifampicin Resistance in Mycobacterium smegmatis. PLoS ONE 9, e106016.

Zhang, Y., Heym, B., Allen, B., Young, D., and Cole, S. (1992). The catalase-peroxidase gene and isoniazid resistance of Mycobacterium tuberculosis. 358, 591-593.

Zhang, Y., Dhandayuthapani, S., and Deretic, V. (1996). Molecular basis for the exquisite sensitivity of Mycobacterium tuberculosis to isoniazid. Proc. Natl. Acad. Sci. U. S. A. 93, 13212-13216.

Van Zyl, J.M., and Van Der Walt, B.J. (1994). Apparent hydroxyl radical generation without transition metal catalysis and tyrosine nitration during oxidation of the anti-tubercular drug, isonicotinic acid hydrazide. Biochem. Pharmacol. 48, 2033-2042. 


\section{Appendix}

\subsection{Solutions}

All solutions with the exception of proteinase $\mathrm{K}$ were stored at room temperature. Proteinase $\mathrm{K}$ was stored at $-20^{\circ} \mathrm{C}$.

\section{Tris- $\mathrm{HCl}(\mathrm{pH} 7.5)$}

$121.1 \mathrm{~g}$ of Tris Base was dissolved in $900 \mathrm{~mL}$ deionised water, and the $\mathrm{pH}$ adjusted to 7.5 with the addition of $\mathrm{HCl}$. Once at the desired $\mathrm{pH}$ the volume was topped up to $1 \mathrm{~L}$.

\section{$0.5 \mathrm{M}$ EDTA (pH 8.0)}

186.2 $\mathrm{g}$ of EDTA was added to $900 \mathrm{~mL}$ deionised water, and the $\mathrm{pH}$ adjusted to 8.0 with the addition of $\mathrm{NaOH}$. Once at the desired $\mathrm{pH}$ the volume was topped up to $1 \mathrm{~L}$.

\section{TE Buffer}

A 10x stock of TE buffer was made up containing $100 \mathrm{mM}$ Tris- $\mathrm{HCl}$ and $10 \mathrm{mM}$ EDTA in deionised water and sterilised by autoclaving at $121{ }^{\circ} \mathrm{C}$ for 30 minutes on a liquid cycle. Working stocks of $1 x$ TE buffer were made up using a $1 / 10$ dilution of the $10 x$ stock in deionised water and sterilised as above.

\section{TAE Buffer}

A 50x stock was made by dissolving $242 \mathrm{~g}$ Tris base in $1 \mathrm{~L}$ of deionised water containing 57.1 $\mathrm{mL}$ glacial acetic acid and $50 \mathrm{mM}$ EDTA. The solution was diluted to a $1 \mathrm{x}$ working stock in deionised water.

\section{CTAB}

$10 \mathrm{~g} \mathrm{CTAB}$ and $4 \mathrm{~g} \mathrm{NaCl}$ were added to $100 \mathrm{~mL}$ deionised water. The solution was heated at $65{ }^{\circ} \mathrm{C}$ in a water bath, with frequent agitation until dissolved. The CTAB solution was then stored at $4{ }^{\circ} \mathrm{C}$.

\section{Proteinase K}

The proteinase $\mathrm{K}$ stock was made up by dissolving $1 \mathrm{mg}$ of proteinase $\mathrm{K}$ powder (Sigma Aldrich) in $1 \mathrm{mM}$ Tris- $\mathrm{HCl}(\mathrm{pH} 8.0$ ) and $1.5 \mathrm{mM}$ calcium acetate in deionised water. 


\section{Resazurin}

The resazurin stock solution was made up as a $0.3 \%(\mathrm{w} / \mathrm{v})$ solution in $\mathrm{ddH}_{2} \mathrm{O}$ and stored at $4{ }^{\circ} \mathrm{C}$. A working solution of resazurin at a concentration of $0.03 \%(\mathrm{w} / \mathrm{v})$ was made up by diluting the stock solution in $\mathrm{ddH}_{2} \mathrm{O}$ and filter sterilising before storing at $4{ }^{\circ} \mathrm{C}$.

\subsection{Routine laboratory procedures}

\section{Cleaning electroporation cuvettes}

Electroporation cuvettes were cleaned for reuse after each transformation. Cuvettes were soaked $12.5 \%$ bleach for 30 minutes with agitation, rinsed in $d_{d d} \mathrm{H}_{2} \mathrm{O}$, soaked in $1 \mathrm{M} \mathrm{HCl}$ for 30 minutes with agitation, rinsed in $\mathrm{dd}_{2} \mathrm{O}$, and then rinsed in $75 \%$ ethanol three times. Cuvettes were dried, and stored. Immediately prior to use cuvettes were sterilised with UV for 10 minutes in a laminar flow hood.

\section{Cleaning 384-pin RePads ${ }^{\circledR}$}

RePads $^{\circledR}$ were cleaned after each use. RePads ${ }^{\circledR}$ were soaked in $12.5 \%$ bleach overnight, and then soaked in a mixture of dishwashing detergent and water overnight. RePads ${ }^{\circledR}$ were then rinsed under tap water and scrubbed thoroughly wish a dish-brush, and then dried on the bench. RePads ${ }^{\circledR}$ were then dipped in $100 \%$ ethanol and left to dry in a laminar flow hood. Dry RePads ${ }^{\circledR}$ were stored in large zip-lock plastic bags.

\section{Cleaning metal 96-pin tool}

The pinning tool was dipped in $12.5 \%$ bleach $(\mathrm{v} / \mathrm{v})$ for one minute, then dipped in $\mathrm{dd}_{2} \mathrm{O}$ for a further minute, before being dipped in $100 \%$ ethanol and flamed to sterilise between pinning each plate, and after use. 


\subsection{MSMEI and MSMEG annotations}

This study used the $M$. smegmatis $\mathrm{mc}^{2} 155$ reference genome using the MSMEI annotations. The older MSMEG annotations are provided for each of the $M$. smegmatis $m^{2} 155$ genes mentioned in this study.

\begin{tabular}{cc}
\hline $\begin{array}{c}\text { MSMEI } \\
\text { annotation }\end{array}$ & $\begin{array}{c}\text { MSMEG } \\
\text { annotation }\end{array}$ \\
\hline 0062 & 0061 \\
0227 & 0234 \\
1186 & 1221 \\
1210 & 1245 \\
1211 & 1246 \\
1800 & 1842 \\
1801 & 1843 \\
1904 & 1946 \\
1905 & 1947 \\
2671 & 2738 \\
2672 & 2739 \\
3093 & 3175 \\
3189 & 3273 \\
3358 & 3437 \\
3380 & 3461 \\
3416 & 3496 \\
3684 & 3773 \\
4123 & 4221 \\
4267 & 4371 \\
4423 & 4536 \\
4424 & 4537 \\
4764 & 4889 \\
4765 & 4890 \\
4815 & 4942 \\
5136 & 5275 \\
5244 & 5392 \\
5460 & 5610 \\
5538 & 5688 \\
5554 & 5705 \\
5621 & 5775 \\
5828 & 5989 \\
6040 & 6201 \\
6092 & 6253 \\
6216 & 6384 \\
6263 & 6430 \\
\hline &
\end{tabular}




\begin{tabular}{ll}
\hline 6264 & 6431 \\
6301 & 6473 \\
6730 & 6919 \\
\hline
\end{tabular}

\subsection{Isoniazid analogues}

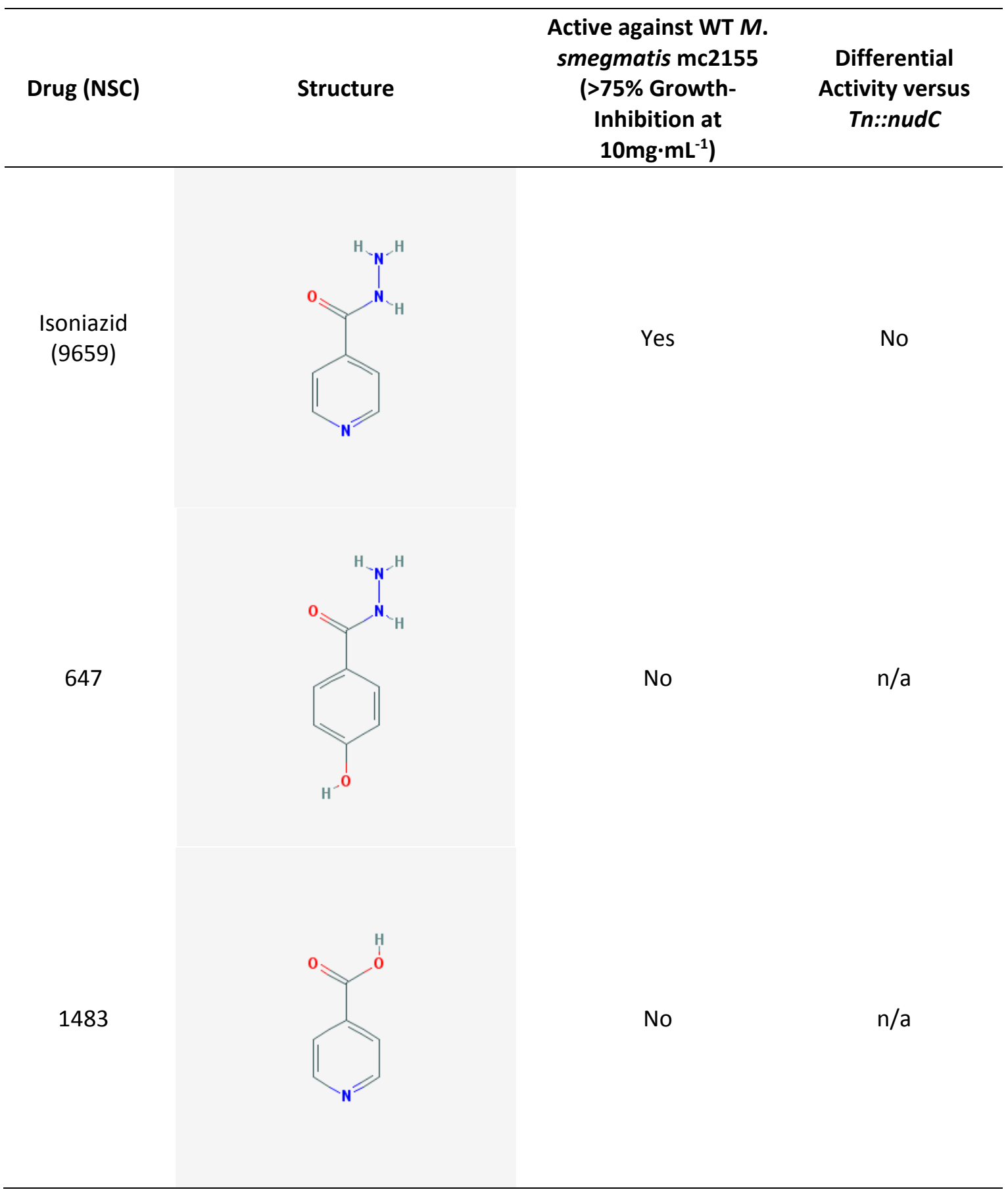




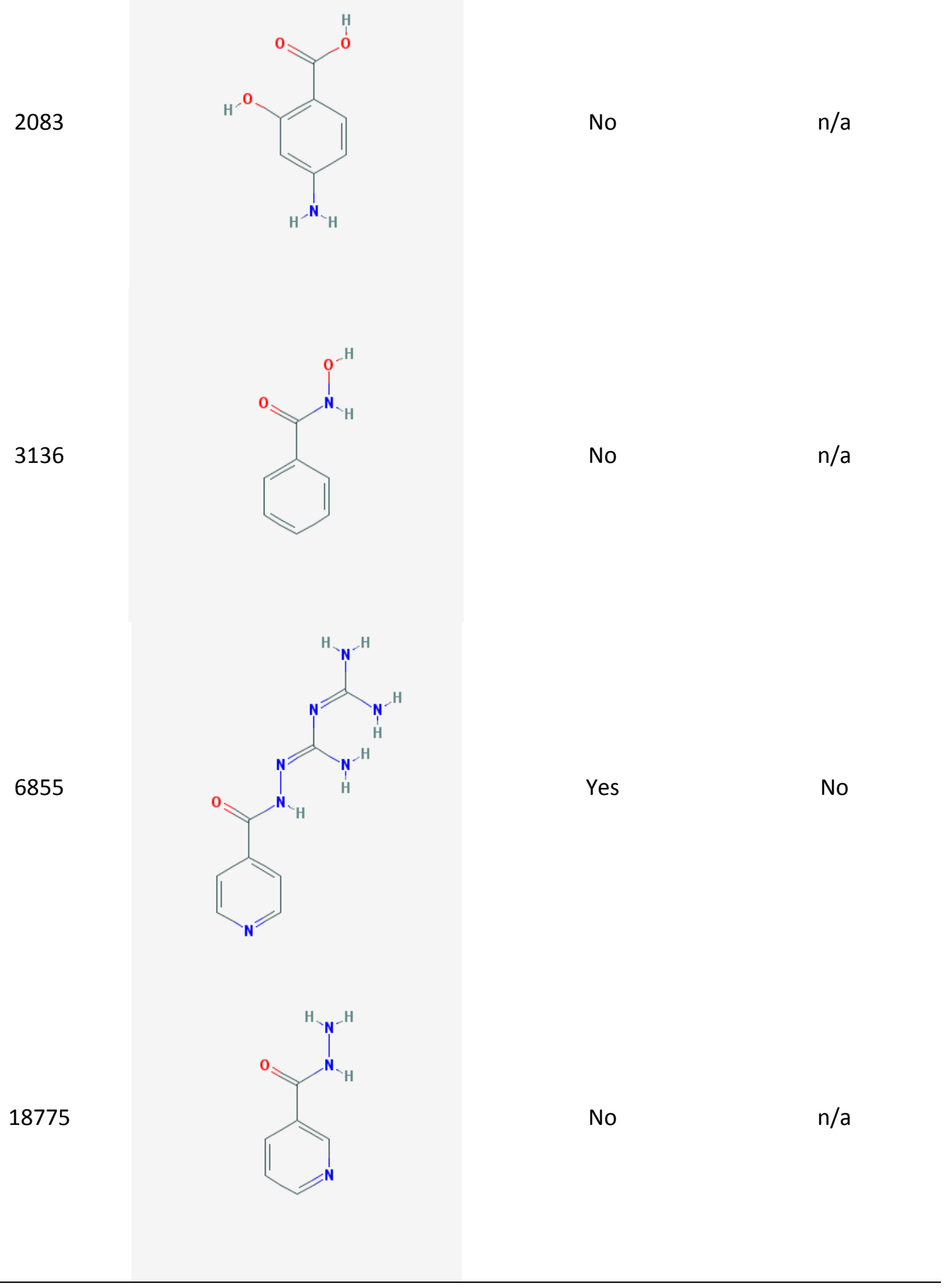


23449

33759

33760

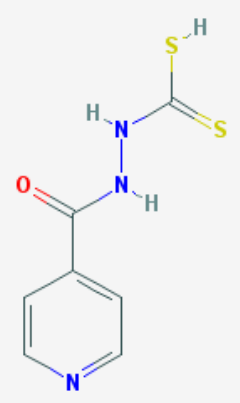

K+

Yes

No
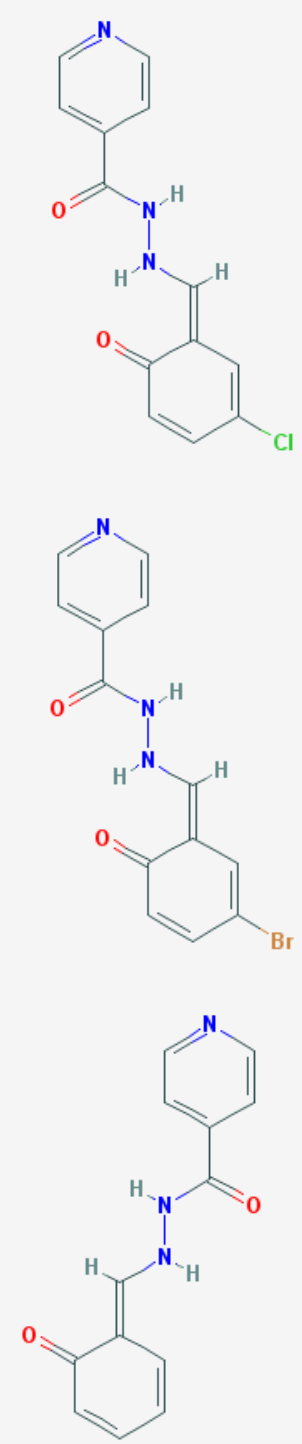

Yes

Yes

Yes

Yes

Yes

No 


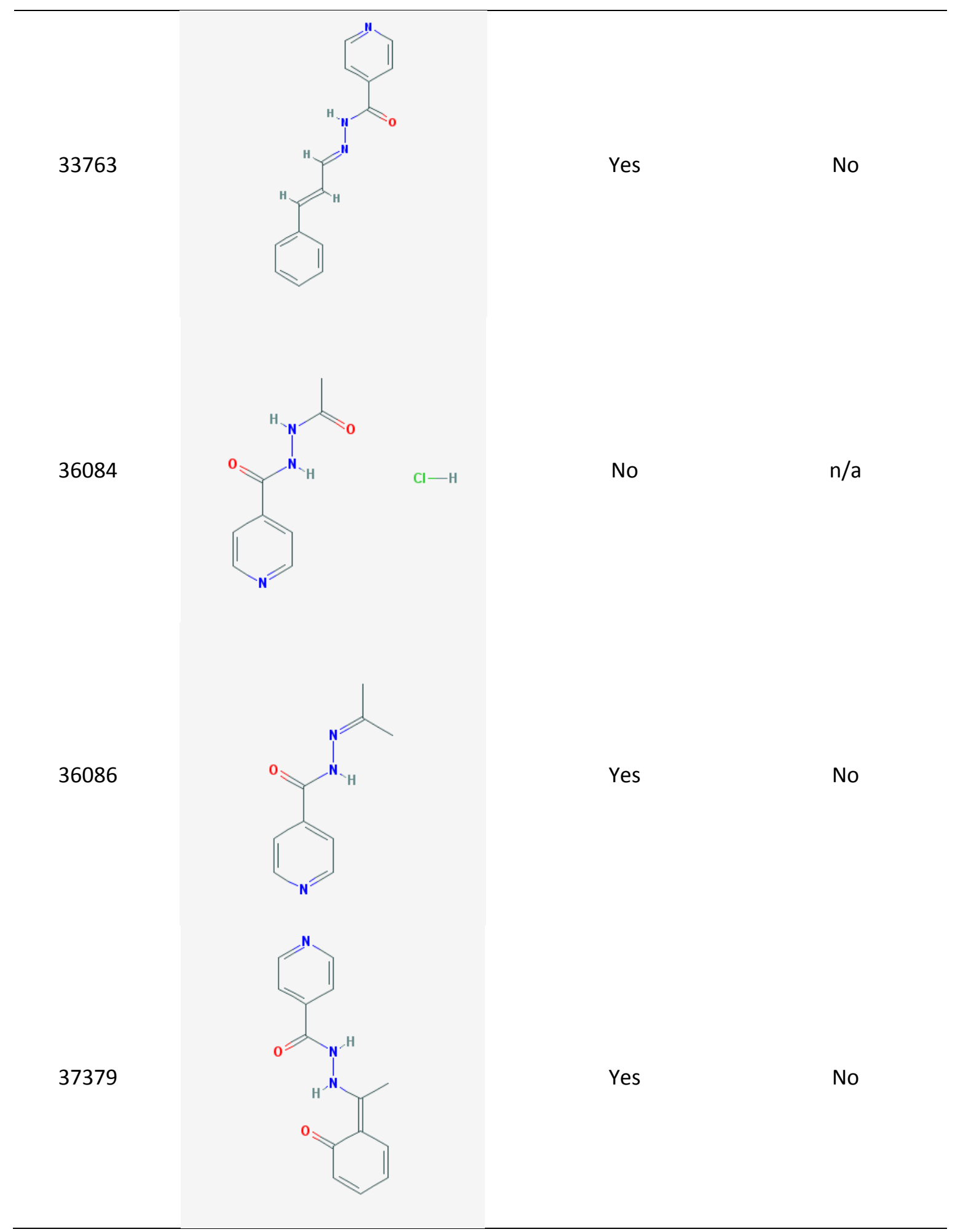




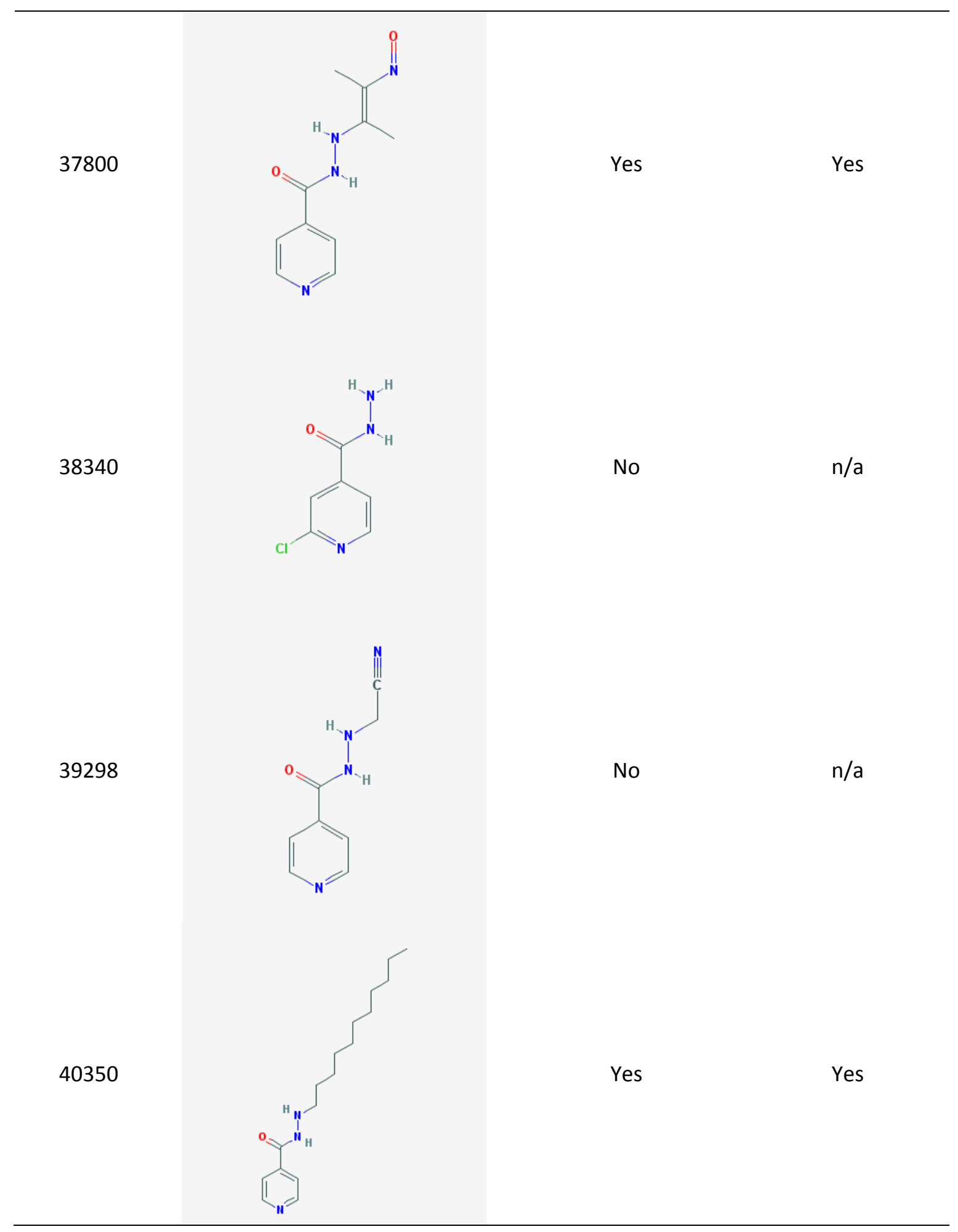




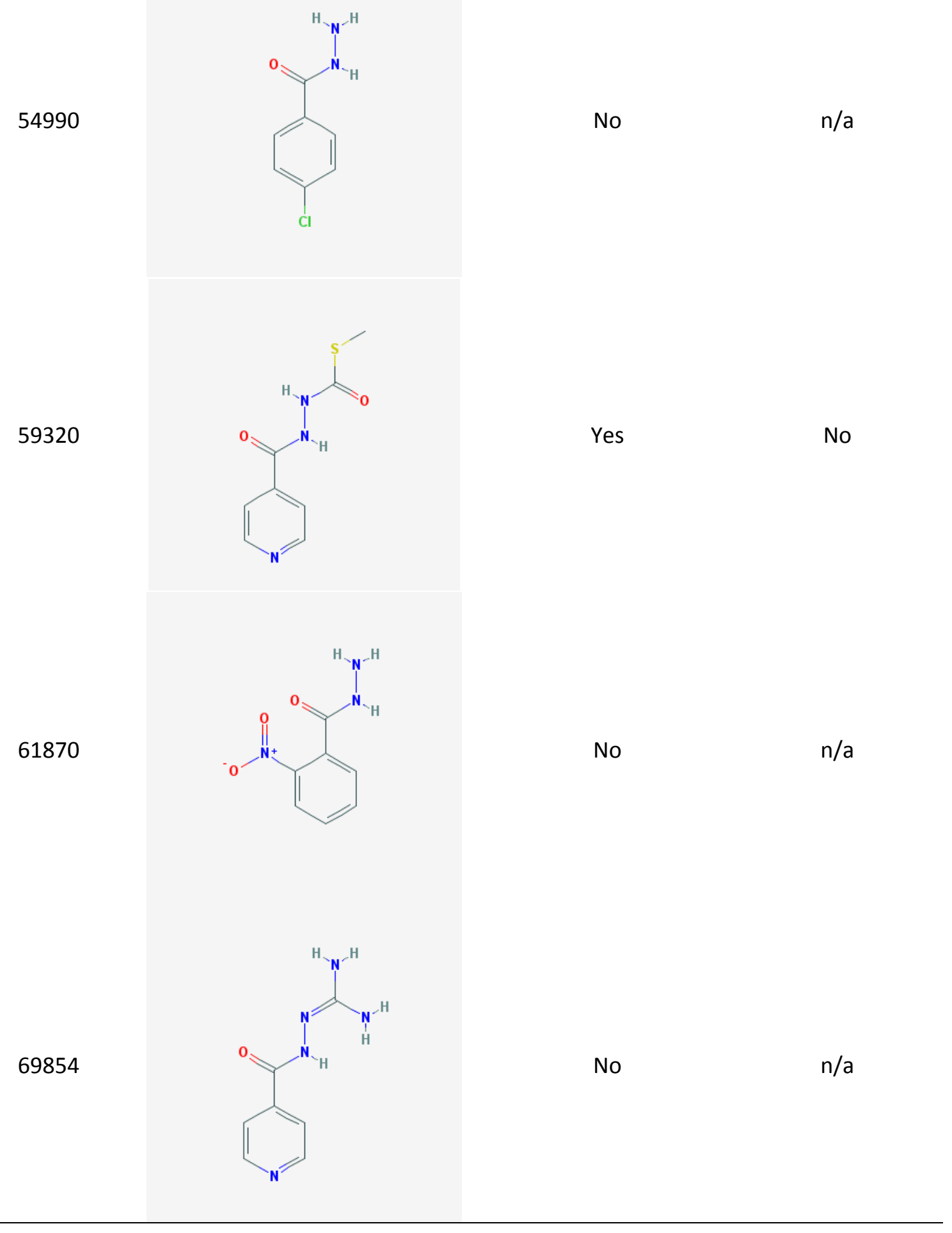


81850

82353

130848

141142

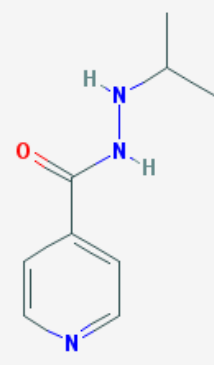

No

n/a

No

$\mathrm{n} / \mathrm{a}$
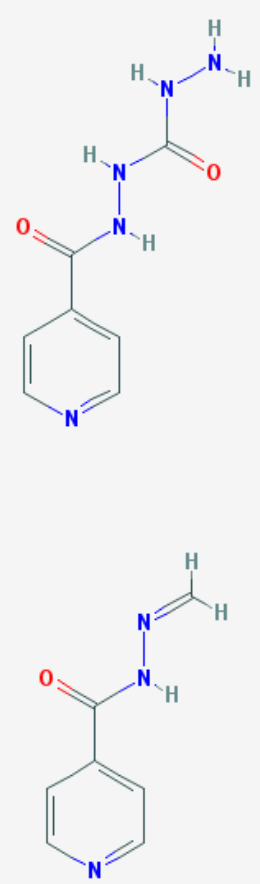

No

n/a

Yes

Yes 


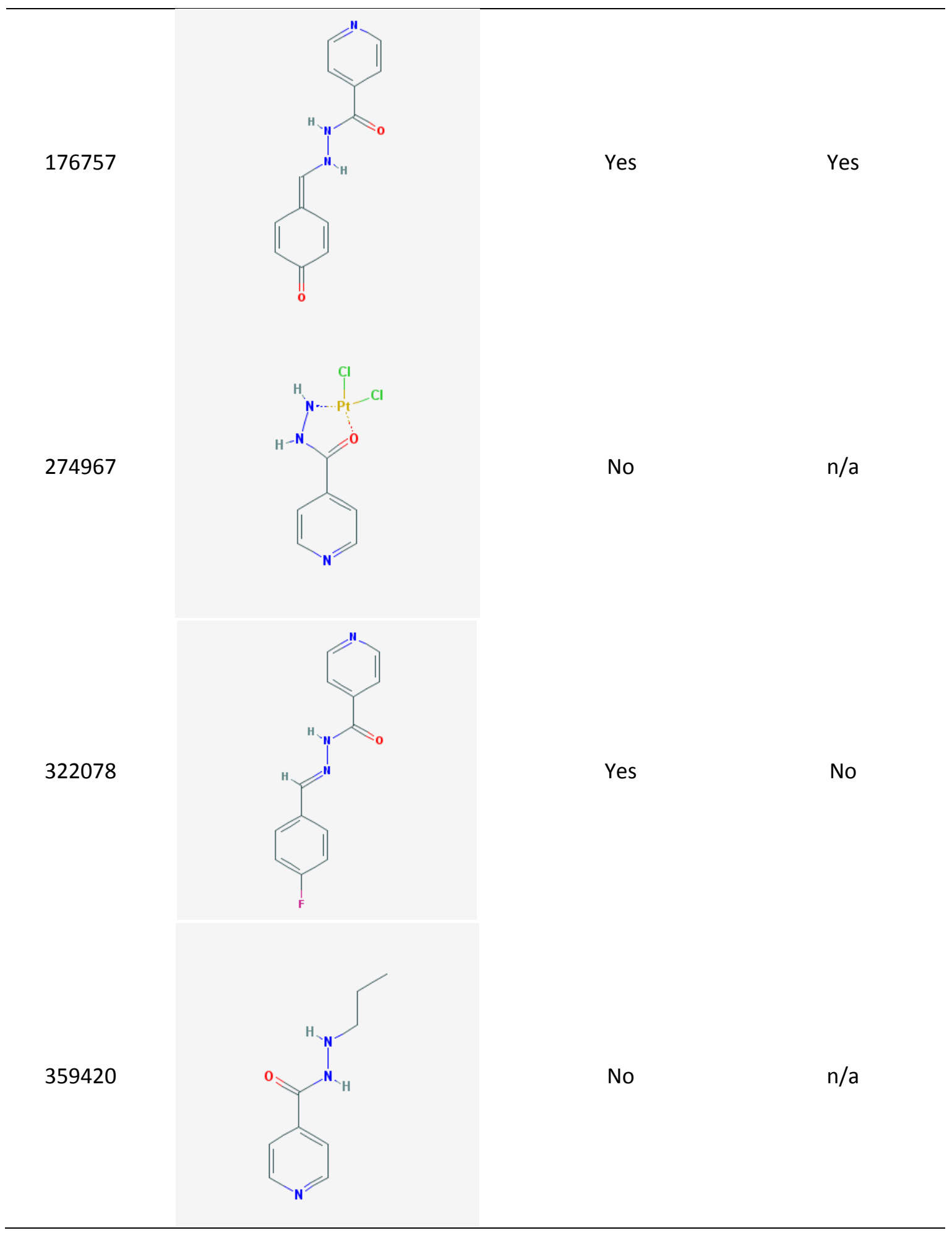




\section{5.katG protein alignments}

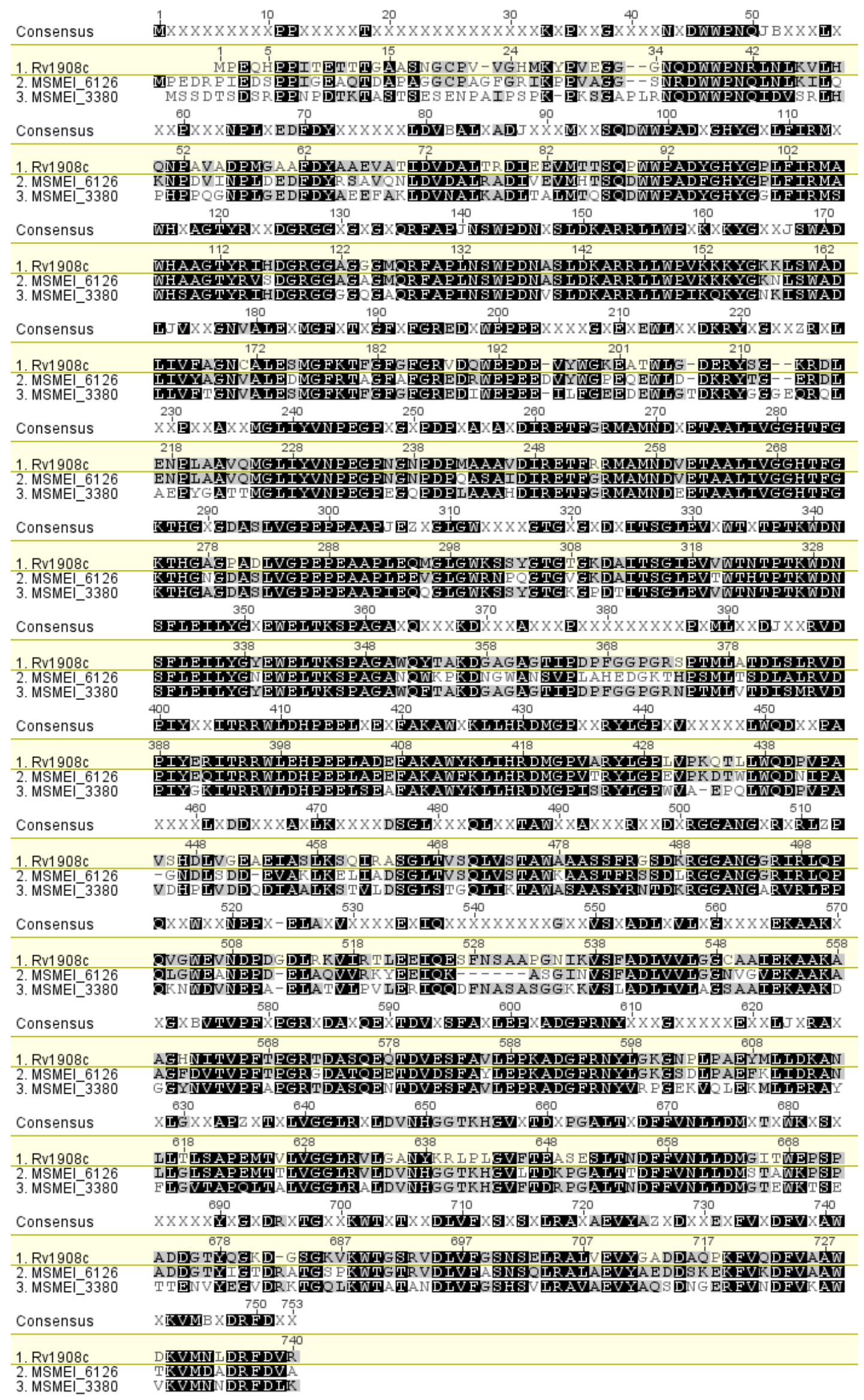




\subsection{Publications}

Campen, R.L., Ackerley, D.F., Cook, G.M., and O'Toole, R.F. (2015). Development of a Mycobacterium smegmatis transposon mutant array for characterising the mechanism of action of tuberculosis drugs: Findings with isoniazid and its structural analogues. Tuberculosis Article In Press. 